\title{
Atomistic simulation study of short pulse laser-induced generation of crystal defects in metal targets
}

\author{
A Dissertation \\ Presented to \\ the faculty of the School of Engineering and Applied Science \\ University of Virginia
}

\author{
in partial fulfillment \\ of the requirements for the degree \\ Doctor of Philosophy
}

by

Eaman Tahir Abdul Karim

May

2016 


\section{APPROVAL SHEET}

The dissertation

is submitted in partial fulfillment of the requirements

for the degree of

Doctor of Philosophy

\section{Eaman Tahir Abdul Karim \\ AUTHOR}

The dissertation has been read and approved by the examining committee:

Leonid V. Zhigilei

Advisor

$\underline{\text { William A. Soffa }}$

$\underline{\text { Petra Reinke }}$

$\underline{\text { James M. Fitz-Gerald }}$

$\underline{\text { Eugene Kolomeisky }}$

Accepted for the School of Engineering and Applied Science:

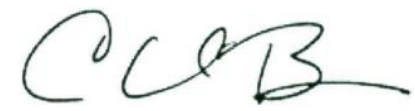

Craig H. Benson, Dean, School of Engineering and Applied Science

May

2016 
To my family

My parents, Mohammed, Hayder, Dina, and Zainab

With your love and support, I could write this document 


\section{Acknowledgements}

I would like to express my deepest thanks and gratitude to my advisor Professor Leonid V. Zhigilei. Since 2008, when I joined the Computational Materials Group (CMG) as a visiting scholar, Professor Zhigilei has been assisting me in developing my professional skills and knowledge. I would like to thank him for his kindness in hosting me in the CMG, his excellent mentoring, and for his professional guidance during my exchange program and later when I joined the PhD program in the Department of Materials Science and Engineering. During all these years, Professor Zhigilei involved me to work in several important research and provides me with the opportunities to present my results in several important conferences as well as helping me in publishing my research in several prestige journals. I would like to thank him for his availability to discuss any problems I encountered during my research. His suggestions helped me to overcome these problems, to improve my scientific thinking and to be more precise in my research. I am extremely grateful to you Leo.

I would like to thank Professor William A. Soffa, Professor Petra Reinke, Professor James M. Fitz-Gerald, and Professor Eugene Kolomeisky for their kind acceptance to serve as members in my dissertation committee. I am extremely grateful to the great assistance by Professor William A. Soffa during my PhD program, thank you so much professor Soffa. I would also like to express my thanks to Professor Kathryn Thornton for her kind assistance during my program.

I also would like to express my deepest thanks to my department, the Department of Materials Science and Engineering represented by the Chair of the department, Professor William C. Johnson, and all the faculty members who helped me to understand and enjoy learning the Materials Science. I also would like to thank all the administrative staff in the Department of Materials Science and Engineering for their wonderful assists during all these years.

I would like to thank all the former and current colleagues in the Computational Materials Group, Zhibin Lin, Alexey Volkov, Marshall Tabetah, Richard Salaway, Chengping Wu, Maxim V. Shugaev, Bernard Wittmaack, Miao He, Cheng-Yu Shih, and Wu Han, for their help and fruitful discussions during my study. Special thanks to Chengping Wu and Maxim V. Shugaev who I enjoyed working with both of them during these years. 
Finally, I would like to thank all my friends in Charlottesville who had made my staying in this wonderful city very enjoyable during all these years. 


\begin{abstract}
Modification of surface structure of metals by short pulse laser irradiation is increasingly used for tailoring surface properties for the needs of practical applications. The rapid energy deposition and shallow heat affected zones in the irradiated metal targets minimize the residual damage and enable micro/nanostructuring of surfaces with good quality and reproducibility. The limited understanding of the underlying physics and interrelations between the processes responsible for laser-induced structural transformations, however, slows down the design of new laser driven techniques for advanced material processing. Under conditions when the fast rates of the laser-induced processes and the small size of laser-modified regions present a challenge for experimental characterization, computational modeling is playing an increasingly important role in the development of the theoretical understanding of the laser-materials interactions and the advancement of laser applications.
\end{abstract}

In this computational study, the microscopic mechanisms and kinetics of fast laserinduced phase transformations in metal targets irradiated by short laser pulses are investigated in large-scale atomistic computer simulations. The computational model used in the simulations combines the classical molecular dynamics method with a continuum description of the laser excitation of conduction band electrons, electron-phonon equilibration, and electron heat conduction in the irradiated metal targets. The characteristics of short pulse laser interaction with metal targets in vacuum and under conditions of spatial confinement by a solid transparent overlayer are investigated in a series of atomistic simulations. Three distinct regimes of the material response to the laser energy deposition are identified in the simulations, namely, the regime of laser-induced melting and solidification of the surface region of the target, the regime of photomechanical spallation, and the so-called "phase explosion regime" characterized by an explosive disintegration of a superheated surface region into a vapor and small liquid droplets. The confinement by a transparent overlayer has strong effect on the laser induced processes, including the decrease in the maximum depth of melting and suppression of the spallation and phase explosion.

The computational investigation of the laser-induced generation of crystal defects reveals the formation of a high density of point defects (mostly vacancies and some interstitials) in the surface regions of the metal targets. The generation of a high density of vacancies have important 
implications on the physical, chemical, and mechanical properties of the surface layers. Computational analysis reveals a strong correlation between the solidification front velocity and the concentration of vacancies, which in turn suggests that the vacancies are mainly generated at the rapidly advancing solidification front under conditions of strong undercooling below the melting temperature of the target material.

The laser-induced generation of dislocations is also observed in the simulations of bcc $\mathrm{Cr}$ targets and is found to have a strong dependence on the crystallographic orientation of the target surface. For (001) Cr targets, a transient appearance of small loops of unstable partial dislocations outlining islands of stacking faults takes place at the initial stage of the relaxation of laser-induced stresses. For (110) and (111) Cr targets, the emission of a high density of perfect dislocations from the melting front is observed a few picoseconds after the laser pulse and is related to the high values of the resolved shear stress generated in many of the 48 slip systems of the bcc structure.

The computational predictions from this study have implications for optimization of the experimental conditions in current and emerging applications based on short pulse laser-induced surface modification. 


\section{Contents}

List of Figures $\quad$ XII

List of Tables $\quad$ XXX

List of Publications $\quad$ XXXI

List of Presentations $\quad$ XXXII

$\begin{array}{ll}\text { Chapter } 1 & 1\end{array}$

1. Introduction 1

1.1 Material modification with laser 1

1.2 Experimental methods of laser material modification 3

1.3 Computational modeling of laser-metals interactions 4

1.3.1 Continuum modeling 5

1.3.2 Atomistic molecular dynamics modeling 9

1.3.3 Combined continuum-atomistic modeling 13

$\begin{array}{ll}1.4 \text { Objectives of this study } & 19\end{array}$

Chapter 2 25

2. Molecular dynamics study of femtosecond laser interactions with Cr targets 25

2.1 Introduction 26

2.2 Computational model 27

2.2.1 Computational setup in small-scale simulations 28

2.2.2 Computational setup in large-scale simulation 28

2.3 Irradiation regimes in femtosecond laser pulse interactions with $\mathrm{Cr}$ targets $\quad 29$

2.3.1 Ablation yield and composition of the ablation plume 29

2.3.2 Mechanisms of melting, spallation, and phase explosion 33 
2.3.3 A microscopic view of the mechanisms of laser-induced photomechanical spallation from a large-scale atomistic simulation

2.4 Summary

Chapter 3

3. Generation of crystal defects in different regimes of laser Interactions with metals

3.1 Introduction

3.2 Computational model

3.2.1 Computational setup for simulations of laser interactions with $\mathrm{Cr}$ targets

3.2.2 Computational setup for simulations of laser interactions with Ni targets

3.3 Generation of point defects below the spallation threshold

3.4 Laser fluence dependence of the vacancy concentration

3.5 Generation of crystal defects in the interactions of short pulse laser with (001), (110), and (111) Cr targets

3.5.1 Generation of partial and perfect dislocations in (001) Cr target

3.5.2 Generation of perfect dislocations in (110) and (111) Cr targets

3.5.3 Analysis of the resolved shear stress in (001), (110), (111) Cr targets

3.5.4 Generation of vacancies and self-interstitials in large-scale simulations of (001), (110), (111) Cr targets 93 
Chapter 4

4. Generation of point defects by rapid solidification of $\mathrm{Ni}$ and $\mathrm{Cr}$ under conditions of strong undercooling 99

4.1 Introduction 100

4.2 Computational model 101

4.3 Results and discussion for (001) interfaces 103

4.3.1 Generation of vacancies in $\mathrm{Cr}$ and $\mathrm{Ni}$ during $\begin{array}{ll}\text { a solidification process } & 103\end{array}$

4.3.2 Effect of the solidification front velocity on the $\begin{array}{ll}\text { concentration of vacancies } & 107\end{array}$

4.3.3 Atomic rearrangements near the liquid-solid interface 114

4.3.4 Vacancy diffusion coefficient and time between the vacancy jumps

4.4 The effect of homogeneous nucleation in the liquid phase on the solidification front velocity

4.4.1 The differences in the solidification front velocity in EAM-FBD Ni and EAM-Johnson Ni 121

$\begin{array}{lll}4.5 & \text { Summary } & 143\end{array}$

$\begin{array}{ll}\text { Chapter 5 } & 144\end{array}$

5. The effect of spatial confinement by transparent overlayer on the laser melting, photomechanical spallation, and phase explosion of metal targets irradiated by short laser pulse

5.1 Atomistic simulation study of short pulse laser interactions with a metal target under conditions of spatial confinement by a transparent overlayer

5.1.1 Introduction 
5.1.2.1 TTM-MD model for Ag target 146

5.1.2.2 Computational representation of the transparent overlayer $\quad 150$

5.1.3 Result and discussion 153

5.1.3.1 Thermal, stress, and spatial confinement in laser-materials interactions 153

5.1.3.2 Melting and solidification under spatial confinement 154

5.1.3.3 Suppression of laser spallation by the overlayer 158

5.1.3.4 Suppression of phase explosion by the overlayer 162 5.1.4 Summary 171

5.2 Experimental characterization and atomistic modeling of interfacial void formation and detachment in short pulse laser-processing of metal surface covered by transparent overlayers 173

5.2.1 Introduction 173

5.2.2 Experimental characterization of laser modification of metal-overlayer targets 174

5.2.3 Atomistic simulation of interfacial void formation 178

5.2.3.1 Computational model 178

5.2.3.2 Generation of the interfacial voids and overlayer detachment 179

5.2.3.3 The effect of pre-existing interface voids $\quad 187$

$\begin{array}{ll}5.2 .4 \text { Summary } & 189\end{array}$

6. Summary of the dissertation 191

7. References 196 


\section{List of Figures}

Fig. 1.1. SEM image of holes drilled in a $100 \mu \mathrm{m}$ thick steel foil with (a) $200 \mathrm{fs}, 120 \mu \mathrm{J}, F=0.5$ $\mathrm{J} / \mathrm{cm}^{2}$, (b) $80 \mathrm{ps}, 900 \mu \mathrm{J}, F=3.7 \mathrm{~J} / \mathrm{cm}^{2}$, and (c) $3.3 \mathrm{~ns}, 1 \mathrm{~mJ}$, and $F=4.2 \mathrm{~J} / \mathrm{cm}^{2}$. All the three cases are irradiated by laser pulses at wavelength of $780 \mathrm{~nm}$. Figures are from Ref. [28].

Fig. 1.2. Three distinct relaxation phases of optically excited electrons in metals. The energy distributions inside the material are indicated on the right. Schematic drawing is based on the discussion of Ref. Ref. [87].

Fig. 1.3. Schematic of the TTM-MD model for simulation of laser interaction with metal targets. The laser irradiation is directed from the right side of the figure and periodic boundary conditions are used in the lateral (parallel to the irradiated surface) direction. Spatial discretization in the continuum part of the model and the dimensions of the atomistic and continuum regions are not drawn to the scale. Figure is from Ref. [24].

Fig. 2.1. Total ablation yield (a) and number of individual atoms in the ablation plume (b) as functions of absorbed laser fluence predicted in simulations for bulk $\mathrm{Cr}$ targets irradiated with 200 fs laser pulses. The values of the total yield and the yield of individual atoms are expressed in units of depth in the initial target (number of ejected atoms is equivalent to the number of atoms in a layer of this depth in the initial target). The dashed lines mark the approximate values of the threshold fluences for surface melting, spallation and phase explosion.

Fig. 2.2. Temperature dependence of the volume of a computational cell in MD simulations of a slow constant-pressure heating of a cubic system of liquid $\mathrm{Cr}$. The results of the simulations are used to determine the threshold temperature for the onset of phase explosion in the EAM Cr material.

Fig. 2.3. Contour plots of the spatial and temporal evolution of (a) lattice temperature and (b) pressure in a TTM-MD simulation of a bulk $\mathrm{Cr}$ target irradiated with a $200 \mathrm{fs}$ laser pulse at an absorbed fluence of $85 \mathrm{~J} / \mathrm{cm}^{2}$. The laser pulse is directed along the $\mathrm{Y}$ axes, from the top of the contour plots as shown by the red arrow. The black solid lines separate the melted and crystalline regions of the target. The red solid lines separate the continuum (TTM) and atomistic (TTMMD) parts of the computational system. 
Fig. 2.4. Snapshots of atomic configurations obtained in a TTM-MD simulation of a bulk Cr target irradiated with a $200 \mathrm{fs}$ laser pulse at an absorbed fluence of $85 \mathrm{~J} / \mathrm{cm}^{2}$. The snapshots are taken along the [100] view direction. The atoms are colored according to their potential energy; the red region corresponds to the elevated average atomic energy in the melted region. 35

Fig. 2.5. Contour plots of the spatial and temporal evolution of (a) lattice temperature and (b) pressure in a TTM-MD simulation of a bulk Cr target irradiated with a $200 \mathrm{fs}$ laser pulse at an absorbed fluence of $106 \mathrm{~J} / \mathrm{cm}^{2}$, just above the spallation threshold. The laser pulse is directed along the $\mathrm{Y}$ axes, from the top of the contour plots. The black solid lines separate the melted and crystalline regions of the target. The red solid lines separate the continuum (TTM) and atomistic (TTM-MD) parts of the computational system.

Fig. 2.6. Contour plots of the spatial and temporal evolution of (a) lattice temperature and (b) pressure in a TTM-MD simulation of a bulk Cr target irradiated with a $200 \mathrm{fs}$ laser pulse at an absorbed fluence of $298 \mathrm{~J} / \mathrm{cm}^{2}$, just above the threshold for the phase explosion. The laser pulse is directed along the $\mathrm{Y}$ axes, from the top of the contour plots. The black solid lines separate the melted and crystalline regions of the target. The red solid lines separate the continuum (TTM) and atomistic (TTM-MD) parts of the computational system. Marks A, B, and C show the time and locations in the ablation plume for which snapshots of atomic configurations are shown in Fig. 2.7.

Fig. 2.7. Snapshots of atomic configurations obtained in a TTM-MD simulation of a bulk $\mathrm{Cr}$ target irradiated with a 200 fs laser pulse at an absorbed fluence of $298 \mathrm{~J} / \mathrm{cm}^{2}$, close to the threshold for the phase explosion. Snapshots are taken at times and locations marked in the temperature contour plot in Fig. 2.6 (a). The snapshots are taken along the [010] view direction.

Fig. 2.8. Contour plots of the spatial and temporal evolution of (a) the lattice temperature, (b) pressure, and (c) density in a large scale TTM-MD simulation of a bulk (001) Cr target irradiated with a $50 \mathrm{fs}$ laser pulse at an absorbed laser fluence of $1100 \mathrm{~J} / \mathrm{m}^{2}$, slightly above the spallation threshold. The laser pulse is directed along the Y-axis as shown by the red arrow in (a). The black line separates the melted region from the crystalline part of the $\mathrm{Cr}$ target. 
Fig. 2.9. Snapshots of atomic configurations obtained in a TTM-MD simulation of laser-induced photomechanical spallation of a bulk (001) Cr target irradiated with a $50 \mathrm{fs}$ laser pulse at an absorbed laser fluence of $1100 \mathrm{~J} / \mathrm{m}^{2}$. The laser irradiation is directed from the top of the images. The atoms are colored according to their potential energies: the dark blue region represents the bcc $\mathrm{Cr}$ structure, green region represents the liquid phase, and the atoms at the top surface of the target and at the internal surfaces appearing in the spallation process are colored red. The light blue stripes that appear in the crystalline part of $\mathrm{Cr}$ target by the time of $50 \mathrm{ps}$ and gradually disappear at later time represent the unstable stacking faults transiently appearing in the system. The snapshots present the evolution of the voids up to the time of a complete spallation of a liquid layer from the bulk of the target.

Fig. 2.10. Snapshot of an atomic configuration obtained in a TTM-MD simulation of laserinduced photomechanical spallation of a bulk (001) Cr target irradiated with a $50 \mathrm{fs}$ laser pulse at an absorbed laser fluence of $1100 \mathrm{~J} / \mathrm{m}^{2}$. The snapshot is shown for a time of $800 \mathrm{ps,} \mathrm{when} \mathrm{the}$ top liquid layer is completely separated from the bulk of the target. The snapshot illustrates the formation of nine solidified spikes with the length ranged between 12 and $130 \mathrm{~nm}$. The atoms are colored according to their potential energies with the same coloring scale that is in Fig. 2.9.

Fig. 2.11. Surface topography of metal targets obtained in (a) TTM-MD simulation of a bulk (001) Cr target irradiated with a 50 fs laser pulse at an absorbed laser fluence of $1100 \mathrm{~J} / \mathrm{m}^{2}$, and (b) SEM imaging of a copper target irradiated with a $65 \mathrm{fs}$ single pulse at an absorbed laser fluence of $3500 \mathrm{~J} / \mathrm{m}^{2}[18,43]$. The computational and experimental results demonstrate the same characteristic surface features, such as nanoprotrusions, nanocavities, nanopores, and nanorims. The atoms in (a) are colored according to their elevation with respect to the initial surface.

Fig. 2.12. Snapshots of the atomic configuration obtained in TTM-MD simulations of (a) a bulk (001) Cr target irradiated with a 50 fs laser pulse at an absorbed laser fluence of $1100 \mathrm{~J} / \mathrm{m}^{2}$, and (b) a bulk (001) Ag target irradiated with a 100 fs laser pulse at an absorbed laser fluence of 900 $\mathrm{J} / \mathrm{m}^{2}$ [59]. The snapshots are taken after a complete spallation of one liquid layer as well as a complete solidification process of the surface region of the target. The formation of nine spikes 
in $\mathrm{Cr}$ as compared to only one spike in Ag targets may be related to the differences in the surface tension between the two metals. The surface tension for Ag at melting temperature is $\sim 890 \mathrm{mN}$ $\mathrm{m}^{-1}$ (c) [175], which is less than the one in pure $\mathrm{Cr}, 1780 \mathrm{mN} \mathrm{m}^{-1}$ (d) [176].

Fig. 3.1. Snapshots of atomic configurations obtained in TTM-MD simulations of (a) bulk EAM Cr target irradiated with 200 fs laser pulse at an absorbed laser fluence of $850 \mathrm{~J} / \mathrm{m}^{2}$, and (b) bulk EAM Ni target irradiated with 1 ps laser pulse at an absorbed laser fluence of $645 \mathrm{~J} / \mathrm{m}^{2}$. The two laser fluences correspond to the regime of melting and resolidification for the two targets. The snapshots are quenched for 1 ps in order to reduce the thermal noise in the atomic positions and energies. The atoms are colored according to their potential energies. All the atoms that belong to bcc and fcc crystal structure in the $\mathrm{Cr}$ and $\mathrm{Ni}$ targets and have low potential energy are blanked, and only atoms with potential energy higher than $-3.99 \mathrm{eV}$ for EAM Cr and $-4.35 \mathrm{eV}$ for EAM Ni are shown. These atoms correspond either to the liquid phase or to the point defects that remain in the system during the solidification process. The snapshots are taken along the [010] view direction down to the depth of $38 \mathrm{~nm}$ for $\mathrm{Cr}$ and $19 \mathrm{~nm}$ for $\mathrm{Ni}$ targets from the level of the initial surface. The defect configurations labeled as "A" are vacancies, and " $\mathrm{B}$ " is a selfinterstitial, while "C" marks a cluster of 25 vacancies that are generated in the Ni target during the solidification process.

Fig. 3.2. Distribution of vacancies in the surface region of the bulk EAM Cr (a) and EAM Ni (b) targets irradiated by short laser pulses at laser fluencies above the threshold for surface melting. Snapshots of the atomic configurations used in the analysis are shown in Fig. 3.1. Each gray bar in the histograms is the result of the averaging over ten individual (001) atomic planes. The red bars in (b) represent contribution from the six clusters of vacancies that are detected in the $\mathrm{Ni}$ target. The dashed lines mark the depth of the region that experienced transient melting and solidification in response to the laser irradiation.

Fig. 3.3. Concentration of vacancies and the temperature of the surface region of the target at the end of the solidification process as functions of the absorbed laser fluence predicted in TTM-MD simulations of EAM Cr target irradiated by 200 fs laser pulse. The vertical dash-dotted lines mark the approximate values of the threshold fluences for the onset of laser melting, photomechanical spallation, and explosive decomposition of the surface region into vapor and liquid droplets (phase explosion). The range of fluences where the photomechanical spallation is 
the dominant mechanism of the material ejection is highlighted by the light blue color. The concentration of vacancies is averaged over for the region that has experienced transient melting and solidification. The red dashed lines show the mean of the data sets excluding the first two points (below and just above the melting threshold).

Fig. 3.4. Concentration of vacancies and the temperature of the surface region of the target at the end of the solidification process as functions of the absorbed laser fluence predicted in TTM-MD simulations of EAM Ni target irradiated by 1 ps laser pulse. The vertical dash-dotted lines mark the approximate values of the threshold fluences for the onset of laser melting, photomechanical spallation, and explosive decomposition of the surface region into vapor and liquid droplets (phase explosion). Due to the incomplete solidification process in the phase explosion regime, the results of the phase explosion regime are not shown in this figure. The range of fluences where the photomechanical spallation is the dominant mechanism of the material ejection is highlighted by the light blue color. The concentration of vacancies is average over the region that experienced transient melting and solidification. The red dashed lines show the mean of the data sets excluding the first point.

Fig. 3.5. Contour plots of the spatial and temporal evolution of the temperature (left) and pressure (right) in the simulations of bulk (a) (001) $\mathrm{Cr}$, (b) (110) $\mathrm{Cr}$, and (c) (111) $\mathrm{Cr}$ targets irradiated with a $50 \mathrm{fs}$ laser pulse at an absorbed laser fluence of $800 \mathrm{~J} / \mathrm{m}^{2}$. The laser pulses are directed along the $Y$-axis from the top of the contour plots. Black lines separate the melted regions from the crystalline parts of the targets.

Fig. 3.6. Surface topographies of (001) Cr, (110) Cr, and (111) Cr targets irradiated by a $50 \mathrm{fs}$ laser pulse at an absorbed of $800 \mathrm{~J} / \mathrm{m}^{2}$, which is $66 \%$ above the melting threshold. The surface topography is shown after complete solidification of the surfaces, for $270 \mathrm{ps}$ after the laser pulse. The atoms are colored according to their elevation form the arithmetic mean of the highest and lowest elevation points.

Fig. 3.7. Snapshots of atomic configurations obtained in a large-scale TTM-MD simulation of a bulk (001) Cr target irradiated with a 50 fs laser pulse at an absorbed laser fluence of $800 \mathrm{~J} / \mathrm{m}^{2}$, which is $66 \%$ above the threshold for surface melting. The snapshots are quenched to $0 \mathrm{~K}$ in order to reduce thermal noise in atomic positions and energies. Atoms are colored according to 
their potential energy and all the atoms that have energies below $-3.99 \mathrm{eV}$ and belong to the original bcc crystal structure are blanked to display the crystal defects. The red atoms in the top layer of the snapshots belong to the free surface of the target while the atoms in the green region $(30,70$, and $180 \mathrm{ps})$ represent a melted region. The crystal defects marked by $\mathrm{A}$ in a snapshot shown for 30 ps correspond to the stacking faults, while the ones marked by B in a snapshot shown for 270 ps are point defects, vacancies and self-interstitials, that remain in the top surface of the target after complete resolidification of the surface region.

Fig. 3.8. (a) Close up view of the atomic configurations that correspond to the stacking faults observed in the TTM-MD simulation of (001) a Cr target irradiated by a 50 fs laser pulse at an absorbed laser fluence of $800 \mathrm{~J} / \mathrm{m}^{2}$. The atoms are colored according to their potential energies and all the atoms that belong to the original bcc crystal structure and have a potential energy below $-3.99 \mathrm{eV}$ are blanked. The snapshot is taken at a time of $90 \mathrm{ps}$ after the laser pulse and shows that the stacking faults are represented by pairs of atomic planes oriented at $\pm 45^{\circ}$ with respect to the uniaxial expansion along [001] direction. The stacking faults appear in the system when a part of the crystal is shifted along $\{110\}$ planes by a displacement vectors of a/8 $<011>$. The schematic diagram of the atomic arrangement on a $(0 \overline{1} 1)$ plane shown in (b), represent the prediction of the appearance of the stacking faults within the hard-sphere model, where the red circles represent the top layer. The possible formation of the stacking faults, from the hardsphere model, is due to the dissociation of the perfect dislocation with a Burgers vector represented by the red dashed line along [ $[\overline{1} 11]$ into [011] and [ $\overline{2} 11]$ displacements represented by the solid black arrows. The schematic diagram is taken from Ref. [190], the dashed black line along [011] marks the stacking faults at $X=1 / 8$, for the stacking faults that appear in the TTMMD simulation. The diagram in (c) represent the result of the calculation of the generalized stacking faults energy, $\gamma_{\mathrm{GSF}}$, for the (100) slip plane in bcc EAM $\mathrm{Cr}$ as a function of the magnitude of fault vector, $\vec{u}=X \times[\overline{1}, 01+\alpha]$. The GSF curves are calculated for different values of the uniaxial strain, $\alpha$, applied to the bcc lattice in the [001] direction. The line marks the stacking faults at $X=1 / 8$, predicted with the hard-sphere model. The diagram in (c) is taken from Ref. [129].

Fig. 3.9. Snapshots of the atomic configurations obtained in TTM-MD simulation of a bulk $\mathrm{Cr}$ target irradiated with a $50 \mathrm{fs}$ laser pulse at an absorbed laser fluence of $1100 \mathrm{~J} / \mathrm{m}^{2}$, slightly above 
the threshold of the photomechanical spallation. The configurations are quenched to $0 \mathrm{~K}$ for $2 \mathrm{ps}$ in order to reduce thermal noise from atomic positions and energies. The atoms are colored according to their potential energies, all the atoms that have energies below $-3.99 \mathrm{eV}$ are blanked and only the atoms that belong to either liquid phase (green), surface layer (red), or crystal defects (blue) are shown in the snapshots. The defects configuration marked by A, B, C, and D represent the stacking faults, vacancies and self-interstitials, perfect dislocations, and a perfect dislocation resulting from the interaction of two dislocations in early time that partially annihilate and generate a tail of vacancies. The black dashed square in the snapshot shown for 250 ps represents the position the first dislocation is emitted.

Fig. 3.10. Snapshots of defect structures generated in the surface region of a bulk (001) Cr target irradiated by a $50 \mathrm{fs}$ laser pulse at an absorbed laser fluence of $1100 \mathrm{~J} / \mathrm{m}^{2}$, slightly above the threshold for the transition from melting to the spallation regime. The snapshots are quenched to $0 \mathrm{~K}$ for $2 \mathrm{ps}$ and then the "Dislocation Extraction Algorithm", DXA, is used to define the dislocation lines and to determine their Burgers vectors. The snapshots are taken at different simulation times to illustrate the evolution of the dislocations in the system. Each point in the snapshots represents a dislocation segment and each segment is colored according to its Burgers vector, with blue and light green colors corresponding to Burgers vectors $\frac{a}{2}\langle 111\rangle$ and $a\langle 100\rangle$ Burgers vector.

Fig. 3.11. Snapshots of the atomic configurations obtained in large-scale TTM-MD simulation of a bulk (110) Cr target irradiated with a 50 fs laser pulse at an absorbed laser fluence of 800 $\mathrm{J} / \mathrm{m}^{2}$, which is $66 \%$ above the threshold for surface melting. The snapshots are quenched to $0 \mathrm{~K}$ for $2 \mathrm{ps}$ in order to reduce thermal noise in atomic positions and energies. Atoms are colored according to their potential energies and all the atoms that have energies below $-3.99 \mathrm{eV}$ and belong to the original bcc crystal structure are blanked to display the crystal defects. The red atoms in the top layer of the snapshots belong to the free surface of the target, the green atoms represent the melting region and the blue atoms represent the crystal defects. The crystal defects marked by $A$ in a snapshot shown for 10 ps correspond to the point defects, while the one marked by $B$ in a snapshot shown for 100 ps are the perfect dislocations. 
Fig. 3.12. Snapshots of the atomic configurations obtained in large-scale TTM-MD simulation of a bulk (111) Cr target irradiated with a 50 fs laser pulse at an absorbed laser fluence of 800 $\mathrm{J} / \mathrm{m}^{2}$, which is $66 \%$ above the threshold for surface melting. The snapshots are quenched to $0 \mathrm{~K}$ for $2 \mathrm{ps}$ in order to reduce thermal noise in atomic positions and energies. Atoms are colored according to their potential energies and all the atoms that have energies below $-3.99 \mathrm{eV}$ and belong to the original bcc crystal structure are blanked to display the crystal defects. The red atoms in the top layer of the snapshots belong to the free surface of the target, the green atoms represent the melting region and the blue atoms represent the crystal defects. The crystal defects marked by $A$ in a snapshot shown for 10 ps correspond to the point defects, while the one marked by $B$ in a snapshot shown for 100 ps are the perfect dislocations.

Fig. 3.13. Snapshots of defect structures generated in the surface region of a bulk (110) Cr target irradiated by a $50 \mathrm{fs}$ laser pulse at an absorbed laser fluence of $800 \mathrm{~J} / \mathrm{m}^{2}$, which is $66 \%$ above the threshold for the transition to the melting regime. The snapshots are quenched to $0 \mathrm{~K}$ for 2 ps and then analyzed by using the "Dislocation Extraction Algorithm", DXA, in order to define the dislocation lines and to determine their Burgers vectors. The snapshots are taken at different simulation times to illustrate the evolution of the dislocations in the system. Each point in the snapshots represents a dislocation segment and each segment colored according to its Burgers vector: the blue segments have Burgers vectors of $\frac{a}{2}\langle 111\rangle$ and, the light green ones have Burgers vectors of $a\langle 100\rangle$.

Fig. 3.14. Snapshots of defect structures generated in the surface region of a bulk (111) Cr target irradiated by a $50 \mathrm{fs}$ laser pulse at an absorbed laser fluence of $800 \mathrm{~J} / \mathrm{m}^{2}$, which is $66 \%$ above the threshold for the transition to the melting regime. The snapshots are quenched to $0 \mathrm{~K}$ for 2 ps and then analyzed by using the "Dislocation Extraction Algorithm", DXA, in order to define the dislocation lines and to determine their Burgers vectors. The snapshots are taken at different simulation times to illustrate the evolution of the dislocations in the system. Each point in the snapshots represents a dislocation segment and each segment colored according to its Burgers vector: the blue segments have Burgers vectors of $\frac{a}{2}\langle 111\rangle$ and, the light green ones have Burgers vectors of $a\langle 100\rangle$. 
Fig. 3.15. Enlarged views of elements of dislocation structures generated in the surface region of bulk (110) and (111) Cr targets irradiated by a 50 fs laser pulse at an absorbed laser fluence of $800 \mathrm{~J} / \mathrm{m}^{2}$, which is $66 \%$ above the threshold for the transition to the melting regime. Each point in the snapshots represents a dislocation segment and each segment is colored according to its Burgers vector, with blue and light green colors corresponding to dislocations with Burgers vectors of $\frac{a}{2}\langle 111\rangle$ and $a\langle 100\rangle$, respectively. The configurations shown in (a) and (b) represent interaction between two dislocations with Burgers vector $\frac{a}{2}\langle 111\rangle$ that results in the generation of a dislocation segment with Burgers vector $a\langle 100\rangle$. The configuration in (c) represents a dislocation loops with Burgers vector $\frac{a}{2}\langle 111\rangle$.

Fig. 3.16. Contour plots of the spatial and temporal evolution of the resolved shear stress (left) and pressure (right) obtained in TTM-MD simulations of (001), (110), and (111) Cr targets irradiated with a $50 \mathrm{fs}$ laser pulse at an absorbed laser fluence of $800 \mathrm{~J} / \mathrm{m}^{2}$, which is $66 \%$ above the threshold for melting and solidification. The contour plots of the shear stresses are shown for the slip systems where the highest resolved shear stress is detected, (112)[11 $\overline{1}],(\overline{1} 12)[1 \overline{1} 1]$, (121)[1 $\overline{1} 1]$, for (001), (110), (111) Cr targets, respectively.

Fig. 3.17. Snapshots of the vacancy distributions in the surface regions of bulk (001), (110), and (111) $\mathrm{Cr}$ targets irradiated by a $50 \mathrm{fs}$ laser pulse at an absorbed laser fluence of $800 \mathrm{~J} / \mathrm{m}^{2}$, which is $66 \%$ above the threshold for the transition to the melting regime. The snapshots are taken at the end of the solidification process, $270 \mathrm{ps}$ after the laser pulse, and the atomic configurations are quenches to $0 \mathrm{~K}$ for $2 \mathrm{ps}$. The atoms are colored according to their type, blue atoms surrounded the individual vacancy, green atoms surrounded the di-vacancy occupied the first nearest neighbor, and red atoms surrounding the di-vacancies occupied the second nearest neighbor.

Fig. 3.18. Concentration of vacancies in the surface regions of (a) (001), (b) (110), and (c) (111) $\mathrm{Cr}$ targets irradiated with $50 \mathrm{fs}$ laser pulses at absorbed laser fluences of $800 \mathrm{~J} / \mathrm{m}^{2}$. The gray, dark blue, and light blue diamond symbols represent the concentration of individual vacancies, divacancies occupying the first nearest neighbor lattice sites, and di-vacancies occupying the 
second nearest neighbor lattice sites. The light blue, narrow blue, and gray parts of the plot represent a region that experienced melting and solidification, an intermediate layer, and the rest of the target, respectively. The red dashed lines mark the average concentrations of the individual vacancies in the melting and solidification regions.

Fig. 4.1. Schematic diagram of the thermal treatment of $\mathrm{Cr}$ and $\mathrm{Ni}$ systems that is used in the MD simulation study of the mechanisms responsible for the formation of vacancies under conditions of fixed undercooling.

Fig. 4.2. Snapshots of the atomic configurations obtained in MD simulations of the solidification of liquid-crystal EAM Cr (a) and EAM Ni (b) systems. After the equilibration at the melting temperature, the two systems are rapidly cooled to $0.75 T_{m}$ and the snapshots are shown for two times during the constant-temperature solidification process. The configurations are quenched to $0 \mathrm{~K}$ for $1 \mathrm{ps}$ in order to reduce thermal noise in the atomic positions and energies, and to provide a clear view of the vacancies remaining in the system. The atoms are colored according to their potential energies and all atoms that have low potential energy and belong to bcc or fcc crystal structure are blanked. Only the atoms with potential energy higher than $-3.99 \mathrm{eV}$ for EAM $\mathrm{Cr}$ and $-4.35 \mathrm{eV}$ for EAM Ni are shown in the snapshots. The dashed black rectangles in (a) and (b) represent the regions where the two crystallization fronts meet each other. The snapshots are taken along the $[1 \overline{1} 0]$ view direction. The time in each snapshot represents the time after quenching to the required temperature. In (a), an individual vacancy is represented by a cluster composed of 14 atoms that includes the first eight and the second six nearest neighbors of the empty lattice site. In (b), an individual vacancy is represented by a cluster of 12 atoms that include the first neighbor shell surrounding the vacancy site. 105

Fig. 4.3. Distribution of vacancies in the EAM Cr (a) and EAM Ni (b) systems solidified at a temperature of $0.75 T_{m}$. Each bar in the two histograms represents the vacancy concentration averaged over ten individual (001) atomic planes. In (a), the dark blue region, (1), marks the solid part of in the initial EAM Cr system, and the light blue regions, (2), correspond to the parts that solidified during the adiabatic process used for the generation of liquid-solid coexistence state at $T_{m}$, before quenching the system to the desired temperature. Regions (2) and (3) and (4) represent the liquid phase in the initial liquid-solid $\mathrm{Cr}$ system. In (b), light blue region represents the initial solid phase in the liquid-solid EAM Ni system and the light pink region represents the 
initial liquid phase. In the two systems, the white region, which is also marked as (4) in (a), corresponds to the region where the two solidification fronts meet each other. The solid black lines in (a) represent the initial positions of the two interfaces during the formation of the liquidsolid system, while the dashed lines in (a) and (b) represent the positions of the two interfaces at the liquid-solid coexistence condition, before quenching the system to the desired temperature.

Fig. 4.4. Concentration of vacancies and velocity of solidification front as functions of normalized temperature in EAM Cr system obtained in a series of MD simulations performed at different levels undercooling below the equilibrium melting temperature. The light blue region in both figures corresponds to the undercooling conditions realized in the simulations of laser interactions with the $\mathrm{Cr}$ targets. The gray region represents the amorphous state of the EAM $\mathrm{Cr}$ material, where the glass transition temperature is found to be $T_{g}=810 \mathrm{~K}$. The error bars represent the standard deviations of the concentration of vacancies and the solidification front velocity in the upper and lower figures, respectively. The error bars in both cases are obtained from the results of three sets of simulations, each starting at different initial conditions. The red solid line represents the fitting of the MD results to Eq. (21), while the dashed red line represents the fitting of the MD results to Eq. (22). The black solid and dashed lines represent regions where Eqs. (21) and (22) do not match the results of the simulations.

108

Fig. 4.5. Concentration of vacancies and velocity of solidification front as functions of normalized temperature in EAM Ni system obtained in a series of MD simulations performed at different levels of undercooling below the equilibrium melting temperature. The light blue regions in both figures correspond to the undercooling conditions realized in the simulations of laser interactions with Ni targets. The gray region represents the amorphous state of the EAM Ni material, where the glass transition temperature is $T_{g}=717 \mathrm{~K}$ according to Ref. [179]. The error bars represent the standard deviation of the concentration of vacancies and the solidification front velocity in the upper and lower figures, respectively. The error bars in both cases are obtained from the results of three sets of simulations, each starting with different initial conditions. The red solid line represents the fitting of the MD results to Eq. (21), while the dashed red line represents the fitting of the MD results to Eq. (22). The black solid and dashed 
lines represents the regions where Eqs. (21) and (22) do not match the results of the simulations.

Fig. 4.6. Snapshot of an atomic configuration near the liquid-crystal interface obtained from MD simulation of solidification of the EAM Cr system at $0.50 T_{m}$. The snapshot is taken along [010] view direction and represents eleven atomic planes. The top upper plane is the liquid phase, the second two atomic planes are in the interfacial region and the following eight planes are the solid phase. The atomic configuration is quenched to $0 \mathrm{~K}$ in order to reduce the thermal noise in the atomic positions and energies. The atoms are colored according to the atomic planes they are associated with. The snapshot of the atomic configuration is taken at $102 \mathrm{ps}$ after quenching to $0.50 T_{m}$

Fig. 4.7. Close up view of four atomic planes that, at the initial time of 102 ps include liquid phase plane (plane 242), two interfacial planes (planes 241 and 240), and solid phase plane (plane 239). The atoms are colored according to the atomic planes they are associated with. The atoms with cross ribbons represent the atoms that are going to move to the new atomic plane at a later time and the color of the cross ribbons represent the color of their new atomic plane. The atoms surrounded with ribbons represent the atoms that changed their positions to a new atomic plane, and the color of the ribbons correspond to the color of their initial atomic plane.

Fig. 4.8. The vacancy diffusion coefficient, $D_{v}$, and the time between jumps, $\tau$, predicted in MD simulations for (a) EAM Cr, and (b) EAM Ni systems. The temperature is normalized by the melting temperatures for both systems.

Fig. 4.9. Solidification front velocity as a function of normalized temperature for Ni system where the interatomic interaction between $\mathrm{Ni}$ atoms is described by the EAM-FBD [128] (a) and EAM-Johnson [182] (b) potentials. The error bars in both (a) and (b) represent the standard deviations of the solidification front velocity predicted in three sets of MD simulations, each starting with different initial conditions. The red solid lines represent the fitting of the MD results to Eq. (21), while the dashed red lines represent the fitting of the MD results to Eq. (22). The black solid and dashed lines correspond to regions where Eqs. (21) and (22) cannot provide an adequate description of the computational results. 
Fig. 4.10. Snapshots of the atomic configurations obtained in MD simulations of solidification at $0.6 T_{m}$ in a Ni system described by EAM-FBD (a) and EAM-Johnson interatomic potentials. The snapshots are taken along the [010] view direction. The atomic configurations are quenched to $0 \mathrm{~K}$ for $1 \mathrm{ps}$ to reduce thermal noise in atomic positions and energies. The atoms are colored according to their local structure environment, with the light blue, dark blue and green colors corresponding to the fcc, bcc, and hcp structures, respectively. All the atoms that belong to the liquid phase and have unknown structure environment are blanked.

Fig. 4.11. Snapshots of the atomic configurations obtained in MD simulations of solidification at $0.3 T_{m}$ in a Ni system described by EAM-FBD (a) and EAM-Johnson interatomic potentials. The snapshots are taken along the [010] view direction. The configurations are quenched for $1 \mathrm{ps}$ to reduce thermal noise in atomic positions and energies. The atoms are colored according to their local structure environment, with the light blue, dark blue and green colors corresponding to the fcc, bcc, and hcp structures, respectively. All the atoms that belong to the liquid phase and have unknown structure environment are blanked.

Fig. 4.12. The evolution of the fraction of crystalline regions in the liquid phase predicted in MD simulations of solidification of Ni systems described by (a) EAM-FBD and (b) EAMJohnson potentials. Initially, the two liquid-solid Ni systems are well equilibrated at melting temperature and then quenched to different desired temperatures. The results are shown for six target temperatures: $0.9,0.8,0.7,0.6,0.5$, and $0.3 T_{m}$.

Fig. 4.13. The evolution of the fraction of liquid volume predicted in MD simulations of solidification of Ni systems described by (a) EAM-FBD and (b) EAM-Johnson potentials. Initially, the two liquid-solid $\mathrm{Ni}$ systems are well equilibrated at melting temperature and then quenched to different desired temperatures. The results are shown for six target temperatures: $0.9,0.8,0.7,0.6,0.5$, and $0.3 T_{m}$.

Fig. 4.14. The evolution of the average instantaneous velocity of solidification front (AIV) and average fraction of crystalline regions in the liquid phase (AFC) during the solidification of Ni systems represented by (a) EAM-FBD and (b) EAM-Johnson potentials at a target temperature $0.8 T_{m}$. The error bars in the AIV and AFC represent the standard deviations obtained from three 
series of MD simulations, each starting with different initial conditions. The dashed blue line represents the average instantaneous velocity during the simulation time.

Fig. 4.15. The evolution of the average instantaneous velocity of solidification front (AIV) and average fraction of crystalline regions in the liquid phase (AFC) during the solidification of $\mathrm{Ni}$ systems represented by (a) EAM-FBD and (b) EAM-Johnson potentials at a target temperature $0.6 T_{m}$. The error bars in the AIV and AFC represent the standard deviations obtained from three series of MD simulations, each starting with different initial conditions. The dashed blue line represents the average instantaneous velocity during the simulation time.

Fig. 4.16. The evolution of the average instantaneous velocity of solidification front (AIV) and average fraction of crystalline regions in the liquid phase (AFC) during the solidification of $\mathrm{Ni}$ systems represented by (a) EAM-FBD and (b) EAM-Johnson potentials at a target temperature $0.3 T_{m}$. The error bars in the AIV and AFC represent the standard deviations obtained from three series of MD simulations, each starting with different initial conditions. The dashed blue line represents the average instantaneous velocity during the simulation time.

Fig. 4.17. Instantaneous velocities of the solidification front with respect to the normalized temperature obtained from MD simulations of solidification of Ni systems described by (a) EAM-FBD and (b) EAM-Johnson potentials. Each diagram is divided into three regimes, depending on the major factors affecting the advancement of the solidification front: solidification, homogeneous nucleation, and amorphous regimes. The gray diamonds show the velocity of the solidification front in the regions of undercooled liquid that are free of any crystalline nuclei. The red delta symbols represent the velocity of solidification front at a time when the fraction of crystalline structures in the liquid phase reach 3\%. The blue square symbols represent the solidification front velocity during the last $10 \mathrm{ps}$ of the simulation time. The error bars represent the standard deviation obtained from three series of MD simulations, each starting with different initial conditions.

Fig. 4.18. Concentration of vacancies as a function of normalized temperature in $\mathrm{Ni}$ systems described by (a) EAM-FBD and (b) EAM-Johnson potentials. The error bars represent the standard deviation obtained from three series of MD simulations, each starting with different initial conditions. 
Fig. 5.1. Schematic sketches of two alternative computational setups designed for simulations of laser interactions with a Ag target covered by a transparent overlayer. In both setups, the top layer of the $\mathrm{Ag}$ target with thickness of $L_{T T M-M D}$ is represented by the atomistic TTM-MD model, whereas the heat conduction in the deeper part of the substrate is simulated with TTM equations solved in the region with thickness of $L_{T T M}$. The transparent overlayer is represented by a dynamic imaginary plane in (a) and by a model adopting an atomistic MD description of a part of the overlayer adjacent to the Ag target in (b). The dynamic pressure-transmitting boundary conditions are applied at the bottom of the TTM-MD region and, in (b), at the top of the MD part of the overlayer to mimic non-reflective propagation of laser-induced pressure waves through the boundaries. In both setups, the partial propagation of the laser-induced pressure wave into the silica overlayer and the work of adhesion between the overlayer and the Ag film are reproduced.

Fig. 5.2. Temperature (left) and pressure (right) contour plots in simulations of a bulk Ag target irradiated by a $10 \mathrm{ps}$ laser pulse at a fluence of $900 \mathrm{~J} / \mathrm{m}^{2}$. The irradiated surface is free of confinement in (a) and is covered by a transparent overlayer in $(b, c)$. The overlayer is represented by a dynamic imaginary plane in (b) and by an atomistic MD description of a part of the overlayer adjacent to the Ag target combined with a pressure-transmitting boundary condition in (b). The laser pulse has a Gaussian profile with the peak intensity reached at 25 ps from the beginning of the simulations and is directed along the $\mathrm{Y}$-axis, as shown by the red arrows in the left panels. The black curves separate the melted regions from crystalline parts of the Ag target. The red curves separate the atomistic (TTM-MD) and continuum (TTM) parts of the model representing the Ag targets. The dashed curve in the pressure plot in (a) shows the maximum depth of dislocation propagation. The yellow curves in (b) show the position of the Ag-overlayer interface represented by the dynamic imaginary overlayer.

Fig. 5.3. Temperature (left) and pressure (right) contour plots in simulations of a bulk Ag target irradiated by a $10 \mathrm{ps}$ laser pulse at a fluence of $1000 \mathrm{~J} / \mathrm{m}^{2}$. The irradiated surface is free of confinement in (a) and is covered by a transparent overlayer in (b). The computational setup in the simulation with overlayer is illustrated in Fig. 5.1(b). The laser pulse has a Gaussian profile with the peak intensity reached at $25 \mathrm{ps}$ from the beginning of the simulations and is directed along the Y-axis, as shown by the red arrows in the left panels. The black curves separate the 
melted regions from crystalline parts of the Ag target. The red curves separate the atomistic (TTM-MD) and continuum (TTM) parts of the model representing the Ag targets. The dashed curve in the pressure plot in (a) shows the maximum depth of dislocation propagation. Areas where the density of the material drops below $10 \%$ of the initial density of the solid Ag target are blanked in the plots.

Fig. 5.4. Pressure profiles at a depth of $100 \mathrm{~nm}$ below the surface of Ag targets in simulations performed with and without the transparent overlayer and illustrated by contour plots in Fig. 5.3.

Fig. 5.5. Temperature (left) and pressure (right) contour plots in simulations of a bulk Ag target irradiated by a 10 ps laser pulse at a fluence of $3000 \mathrm{~J} / \mathrm{m}^{2}$. The irradiated surface is free of confinement in (a) and is covered by a transparent overlayer in (b). The computational setup in the simulation with overlayer is illustrated in Fig. 5.1(b). The laser pulse has a Gaussian profile with the peak intensity reached at $25 \mathrm{ps}$ from the beginning of the simulations and is directed along the Y-axis, as shown by the red arrows in the left panels. The black curves separate the melted regions from crystalline parts of the Ag target. Areas where the density of the material drops below $10 \%$ of the initial density of the solid Ag target are blanked in the plots.

163

Fig. 5.6. The evolution of temperature and pressure averaged over atoms that are initially located in the top $5 \mathrm{~nm}$ thick layer of the Ag target irradiated by $10 \mathrm{ps}$ laser pulses at a fluence of $3000 \mathrm{~J} / \mathrm{m}^{2}$ with and without the presence of a transparent overlayer. The points along the trajectories are colored by time up to 250 ps as shown by the legend; from 250 to 850 ps the points are gray with blue lines drawn as a guide to the eye. The black curve shows the temperature-pressure conditions $\left(\mathrm{T}^{*}, \mathrm{P}^{*}\right)$ for the onset of the cavitation or phase explosion in the metastable liquid, calculated for the EAM Ag material as explained in Sect. 5.1.2.1.

Fig. 5.7. Temperature (left) and pressure (right) contour plots in simulations of a bulk Ag target irradiated by a $10 \mathrm{ps}$ laser pulse at a fluence of $6000 \mathrm{~J} / \mathrm{m}^{2}$. The irradiated surface is free of confinement in (a) and is covered by a transparent overlayer in (b). The computational setup in the simulation with overlayer is illustrated in Fig. 5.1(b). The laser pulse has a Gaussian profile with the peak intensity reached at $25 \mathrm{ps}$ from the beginning of the simulations and is directed along the Y-axis, as shown by the red arrows in the left panels. The black curves separate the 
melted regions from crystalline parts of the Ag target. Areas where the density of the material drops below $10 \%$ of the initial density of the solid Ag target are blanked in the plots.

Fig. 5.8. The evolution of temperature and pressure averaged over atoms that are initially located in the top $5 \mathrm{~nm}$ thick layer of the Ag target irradiated by 10 ps laser pulses at a fluence of $6000 \mathrm{~J} / \mathrm{m}^{2}$ with and without the presence of a transparent overlayer. The points along the trajectories are colored by time as shown by the legend. The black curve shows the temperaturepressure conditions $\left(\mathrm{T}^{*}, \mathrm{P}^{*}\right)$ for the onset of the cavitation or phase explosion in the metastable liquid, calculated for the EAM Ag material as explained in Sect. 5.1.2.1.

Fig. 5.9. Concentration profiles and atomic configuration in the region of Ag-overlayer interface at $780 \mathrm{ps}$ after irradiation by a $10 \mathrm{ps}$ laser pulse at a fluence of $6000 \mathrm{~J} / \mathrm{m}^{2}$. The atoms in the snapshot are colored by their type, with blue and red colors used for Ag and overlayer atoms, respectively. In the lower version of the snapshot, the Ag atoms are blanked on the left side of the interface and the overlayer atoms are blanked on the right side of the interface to expose the atomic mixing.

Fig. 5.10. Optical microscope images obtained by focusing microscope lenses on the surface of silica overlayer (a) and the Al-overlayer interface (b) of Al-silica samples irradiated by 10 ps laser pulses at five incident laser fluences indicated in the figure. The black dashed line separates the irradiation conditions that do not result in any visible damage to the overlayer from the ones when cracking/chipping or complete removal of the overlayer is observed. The nature of the target modification is further illustrated by SEM images shown in Fig. 5.11.

Fig. 5.11. SEM images of Al-silica samples irradiated by $10 \mathrm{ps}$ laser pulse at three incident laser fluences of $0.97 \mathrm{~J} / \mathrm{cm}^{2}$ (a-c), $3.84 \mathrm{~J} / \mathrm{cm}^{2}(\mathrm{~d}, \mathrm{e})$, and $7.69 \mathrm{~J} / \mathrm{cm}^{2}(\mathrm{f}, \mathrm{g})$. The images in (a-e) show vertical cross sections of the irradiated spots prepared by FIB milling that reveal the interfacial voids and partial detachment at the metal-overlayer interface. The images in (f,g) show the metal surface exposed by complete removal of the overlayer at a fluence of $7.69 \mathrm{~J} / \mathrm{cm}^{2}$.

Fig. 5.12. Contour plots showing the spatial and temporal evolution of (a) temperature and (b) pressure predicted in a simulation of a Ag-overlayer target irradiated by a 10 ps laser pulse at an absorbed fluence of $0.4 \mathrm{~J} / \mathrm{cm}^{2}$. To ensure complete deposition of the laser energy, the peak intensity of the Gaussian laser pulse is shifted to 25 ps from the beginning of the simulation, as 
shown by the red arrow in (a). The black solid curve line separates the melted region from the crystalline part of the Ag target. The black dashed line tracks the location of the Ag-overlayer interface. The area where the density of the material drops below $10 \%$ of the initial density of the solid Ag target is blanked in the plots. The dashed white rectangle in (a) marks the time range and location of the slice of the computational system for which snapshots of atomic configurations are shown in Fig. 5.13.

Fig. 5.13. Snapshots of atomic configurations obtained in a simulation of a Ag-overlayer target irradiated by a $10 \mathrm{ps}$ laser pulse at an absorbed fluence of $0.4 \mathrm{~J} / \mathrm{cm}^{2}$. Only a $74 \mathrm{~nm}$ thick slices of the computational system adjacent to the Ag-overlayer interface are shown in the snapshots. The atoms are colored according to their potential energies, so that the dark blue color represents liquid Ag, light green and yellow colors represent the solid and melted parts of the overlayer, and the vapor phase atoms are red.

Fig. 5.14. Top-view projections of the voids evolving in the Ag-overlayer interfacial region in the simulation illustrated by Figs. 5.12 and 5.13. The dark regions show the areas where the material density drops below $10 \%$ of the initial density of solid Ag, whereas the white regions represent the connections between the metal substrate and the overlayer.

186

Fig. 5.15. Temperature, pressure and density contour plots obtained in a simulation of bulk Ag substrate covered by a transparent overlayer with an initial $50 \mathrm{~nm}$ thick gap between the overlayer and the Ag substrate. The Ag-overlayer target is irradiated by a $10 \mathrm{ps}$ laser pulse at an absorbed laser fluence of $0.4 \mathrm{~J} / \mathrm{cm}^{2}$. Areas where the density of the material drops below $10 \%$ of the initial density of the solid Ag target are blanked in the plots. The black curves outline voids inside the material and separate the melted region from the crystalline part of the Ag target. The black dashed line shows the position of the Ag-overlayer interface. 


\section{List of Tables}

Table. 3.1: The maximum values of the resolved shear stress calculated for the 48 slip systems in the (001), (110), and (111) Cr targets. The three targets are irradiated with a 50 fs laser pulse at an absorbed laser fluence of $800 \mathrm{~J} / \mathrm{m}^{2}$, which is $66 \%$ above the threshold for melting and solidification. All the values of the maximum resolved shear stress that are above $3 \mathrm{GPa}$ are marked with gray color. The $3 \mathrm{GPa}$ is lower than the theoretical resolved shear stress for $\mathrm{Cr}, 3.8$ GPa.

Table. 3.2: The maximum values of the resolved shear stress calculated for the 48 slip systems in the (001) $\mathrm{Cr}$ targets. The $\mathrm{Cr}$ is irradiated by a $50 \mathrm{fs}$ laser pulse at two absorbed laser fluences, $800 \mathrm{~J} / \mathrm{m}^{2}$, which is $66 \%$ above the threshold for melting and solidification and $1100 \mathrm{~J} / \mathrm{m}^{2}$, which is slightly above the threshold for photomechanical spallation. All the values of the maximum resolved shear stress that are above $3 \mathrm{GPa}$ are marked with gray color. The $3 \mathrm{GPa}$ is lower than the theoretical resolved shear stress for Cr target, 3.8 GPa. 


\section{List of Publications}

1. E. T. Karim, M. V. Shugaev, C. Wu, Z. Lin, H. Matsumoto, M. Conneran, J. Kleinert, R. F. Hainsey, and L. V. Zhigilei, Experimental characterization and atomistic modeling of interfacial void formation and detachment in short pulse laser-processing of metal surfaces covered by transparent overlayers. Submitted to the Appl. Phys. A.

This paper is included in the dissertation, in Chapter 5, Section 5.2.

2. E. T. Karim, C. Wu, and L. V. Zhigilei, Molecular dynamics simulations of lasermaterials interactions: General and material-specific mechanisms of material removal and generation of crystal defects, in Fundamentals of Laser-Assisted Micro- and Nanotechnologies, Springer Series in Materials Science, Vol. 195, Edited by V. P. Veiko and V. I. Konov (Springer International Publishing Switzerland, 2014), pp. 27-49.

3. E. T. Karim, M. Shugaev, C. Wu, Z. Lin, R. F. Hainsey, and L. V. Zhigilei, Atomistic simulation study of short pulse laser interactions with a metal target under conditions of spatial confinement by a transparent overlayer, J. Appl. Phys. 115, 183501, 2014.

This paper is included in the dissertation, in Chapter 5, Section 5.1

4. C. Wu, E. T. Karim, A. N. Volkov, and L. V. Zhigilei, Atomic movies of laser-induced structural and phase transformations from molecular dynamics simulations, in Lasers in Materials Science, Springer Series in Materials Science, Vol. 191, Edited by M. Castillejo, P. M. Ossi, and L. V. Zhigilei (Springer International Publishing Switzerland, 2014), pp. 67-100.

5. E. T. Karim, Z. Lin, and L. V. Zhigilei, Molecular dynamics study of femtosecond laser interactions with Cr targets, AIP Conf. Proc. 1464, 280-293, 2012.

This paper is included in the dissertation, in Chap 2, Section 2.3.1 and 2.3.2.

Several additional papers reporting the results described in Chapters 3 and 4 are in the preparation. 


\section{List of Presentations}

1. Chengping Wu, Maxim V. Shugaev, Eaman T. Karim, and Leonid V. Zhigilei, "Largescale atomistic modeling of structural modification of metal surface in femtosecond laser processing”. The $13^{\text {th }}$ Conference on Laser Ablation (COLA-2015), Cairns, Australia, September 2015.

2. Maxim V. Shugaev, Eaman T. Karim, Cheng-Yu Shih, Chengping Wu, and Leonid V. Zhigilei, "Interaction of Short Pulse Laser Irradiation with Metal Targets under Condition of Spatial Confinement”. APS March Meeting 2015, San Antonio, Texas, March 2015.

3. Chengping Wu, Eaman T. Karim, Maxim V. Shugaev, Cheng-Yu Shih, and Leonid V. Zhigilei, "Large-Scale Atomistic Simulations of Laser Ablation, Generation of Subsurface Voids, Crystal Defects and Nanocrystalline Surface Layers in Short Pulse Laser Processing of Metals". The $9^{\text {th }}$ International Conference on Photo-Excited Processes and Applications, ICPEPA-9, Matsue, Japan, September 2014.

4. Leonid V. Zhigilei, Chengping Wu, Eaman T. Karim, and Maxim V. Shugaev, "LargeScale Atomistic Simulations of Structural Transformations and Microstructure Development in Short Pulse Laser Processing of Metals”. SPIE Solar Energy +Technology, San Diego, California, August 2014.

5. Leonid V. Zhigilei, Chengping Wu, Cheng-Yu Shih, Eaman T. Karim, and Maxim V. Shugaev, "Generation of Nanoparticles in Short Pulse Laser Ablation of Bulk Metal Targets and Thin Films: Insights from Largescale Atomistic Simulations". The $15^{\text {th }}$ International Symposium on Laser Precision Microfabrication, LPM 2014, Vilnius, Lithuania, June 2014.

6. Leonid V. Zhigilei, Chengping Wu, Eaman T. Karim, and Maxim V. Shugaev, "LargeScale Atomistic Simulations of the Structural Transformations and Microstructure Development in Short Pulse Laser Processing of Metals" (Invited talk). European Materials Research Society, E-MRS, Lille, France, May 2014. 
7. Eaman T. Karim, Maxim V. Shugaev, Chengping Wu, Cheng-Yu Shih, Leonid V. Zhigilei, Zhibin. Lin and Robert F. Hainsey, “Atomistic Simulation Study of Short Pulse Laser Interactions with Metal Targets under Conditions of Spatial Confinement". International High Power Laser Ablation Symposium, Santa Fe, NM, April 2014.

8. Eaman T. Karim, Maxim Shugaev, Chengping Wu, Cheng-Yu Shih, Zhibin. Lin, Robert F. Hainsey, and Leonid V. Zhigilei, "Computational and Experimental Study of Short Pulse Laser Ablation under Conditions of Spatial Confinement”. The $12^{\text {th }}$ international Conference on Laser Ablation (COLA 2013), Ischia, Italy, October 2013.

9. Leonid V. Zhigilei, Chengping $\mathrm{Wu}$, and Eaman T. Karim, "Cooling Rates and Mechanisms of Resolidification in Short Pulse Laser Processing of Metal Targets". $12^{\text {th }}$ U.S. National Congress on Computational Mechanics, USNCCM12. Raleigh, North Carolina, July 2013.

10. Chengping Wu, Eaman T. Karim, and Leonid V. Zhigilei, "Atomistic Modeling of Generation of Crystal Defects and Microstructure Development in Short Pulse Laser Processing of Metals” (Invited talk). Fundamental of Laser Assisted Micro-and Nanotechnologies (FLAMN-13)- The Satellite International Symposium of ICONO/LAT2013 Conference, St. Petersburg, Russia, June 2013.

11. Eaman T. Karim, Zhibin. Lin, and Leonid V. Zhigilei, "Atomistic Simulation Study of Femtosecond Laser Interactions with Cr Targets". 3th International Symposium on Laser Precision Microfabrication, Washington, DC, June 2012.

12. Eaman T. Karim, Zhibin. Lin, and Leonid V. Zhigilei, "Molecular Dynamic Study of Femtosecond Laser Interactions with Cr Targets". International Conference on High Power Laser Ablation, Santa Fe, NM, May 2012.

13. Eaman T. Karim, Zhibin. Lin, and Leonid V. Zhigilei, "Generation of point defects in femtosecond laser interaction with Cr targets". 2011 Joint Annual Conference of National Society of Black Physicists and National Society of Hispanic Physicists, Austin, Texas, September 2011. 
14. Leonid V. Zhigilei, Chengping Wu, Eaman T. Karim, and Zhibin. Lin, "Generation of Crystal Defects and Microstructure in Laser Processing of Metals: Molecular Dynamics Study". The $47^{\text {th }}$ Annual Technical Meeting of Society of Engineering Science, Ames, Iowa, October 2010.

15. Leonid V. Zhigilei, Zhibin. Lin, Eaman T. Karim, and Chengping Wu, "Atomistic Simulations of Laser Interactions with Metals: Melting of Nanocrystalline Films and Generation of Crystal Defects" (Invited talk). The $7^{\text {th }}$ International Conference on PhotoExcited Processes and Applications. Copenhagen, Denmark, August 2010.

16. Leonid V. Zhigilei, Zhibin. Lin, Eaman T. Karim, and Chengping Wu, "Atomic-level Simulations of Laser Interactions with Metals: Mechanisms of Melting and Resolidification, Generation of Crystal Defects". The $10^{\text {th }}$ International Conference on Computer Simulation of Radiation Effects in Solids, Krakòw, Poland. July 2010.

17. Eaman T. Karim, Zhibin. Lin, and Leonid V. Zhigilei, "Generation of point defects in femtosecond laser interaction with Cr targets". APS March Meeting, Pittsburgh, Pennsylvania, March 2009. 


\section{Chapter 1}

\section{Introduction}

\subsection{Material modification with laser}

Surface modification in a broad sense includes different types of surface treatments leading to changes in the composition and microstructure of the surface region of the material. Surface processing is commonly used to control optical, mechanical, and chemical properties of the surface and to optimize them for the needs of a given application [1]. The techniques used for surface modification include physical vapor deposition (PVD) [2], chemical vapor deposition (CVD) [3], coating via plasma spraying [4], electrospark deposition [5], ion implantation or sputter deposition [6], ion beam surface modification [7], and electrical discharge machining [8].

Over the past five decades and with the development and advancement of laser systems, such as continuous wave (cw) and pulsed mode lasers [9-14], the special characteristics of the laser light interactions with materials have been acknowledged and utilized in the development of a variety of laser processing techniques. Gradually, the ability of short laser pulses to precisely deposit a large amount of energy into a spatially confined area on a very short timescale received considerable attention and appreciation from the scientific community. Typically, laser irradiation triggers a range of processes that include structural and phase transformations resulting in modification of the properties of a surface region relative to the bulk material [15]. Surface modification of materials often involves laser ablation [16-18], i.e. the process responsible for the removal of a surface layer of the irradiated target. Practical importance of laser ablation is reflected in a broad range of applications based on the laser-induced material removal, including material modification [13, 19, 20], telecommunication [21], energy research [22], etc. The process of laser ablation is controlled by material parameters, such as the absorption mechanism, thermodynamic properties of the material, microstructure, surface morphology, and the presence of crystal defects. Moreover, laser parameters such as wavelength, fluence, spatial and temporal profiles of the laser pulse, repetition rate, and number of pulses are important factors in laser ablation [15]. In particular, the features of ablation process differ according to the laser fluence. At low laser fluences, the characteristics of the ablation process are limited to the material evaporation from the surface region of the target while at high laser 
fluences, the fast laser heating of the material surface may result in homogeneous nucleation and expansion of vapor bubbles, leading to the explosive boiling and ejection of a large amount of vapor phase atoms and liquid droplets [23, 24].

In addition to laser fluence, the pulse duration of the laser is also considered to be an important factor in the laser surface modification. Initially, surface structure has been investigated by using long, nanosecond (ns) and picosecond (ps), laser pulses [25, 26]. Gradually, with the invention of ultrashort laser techniques, such as Colliding Pulse Mode Locking (CPM laser) and Kerr Lens Mode Locking, the advantages of femtosecond (fs) laser pulses have been recognized. Comparative studies of laser material processing elucidated the important characteristics of material processing by fs laser pulses, in contrast to ns and ps laser pulses, which include high precision, minimal heat affected zones, reduction of the debris around the ablated region, and suppression of the plasma formation during the pulse, e.g. ,Fig. 1.1 [2733].
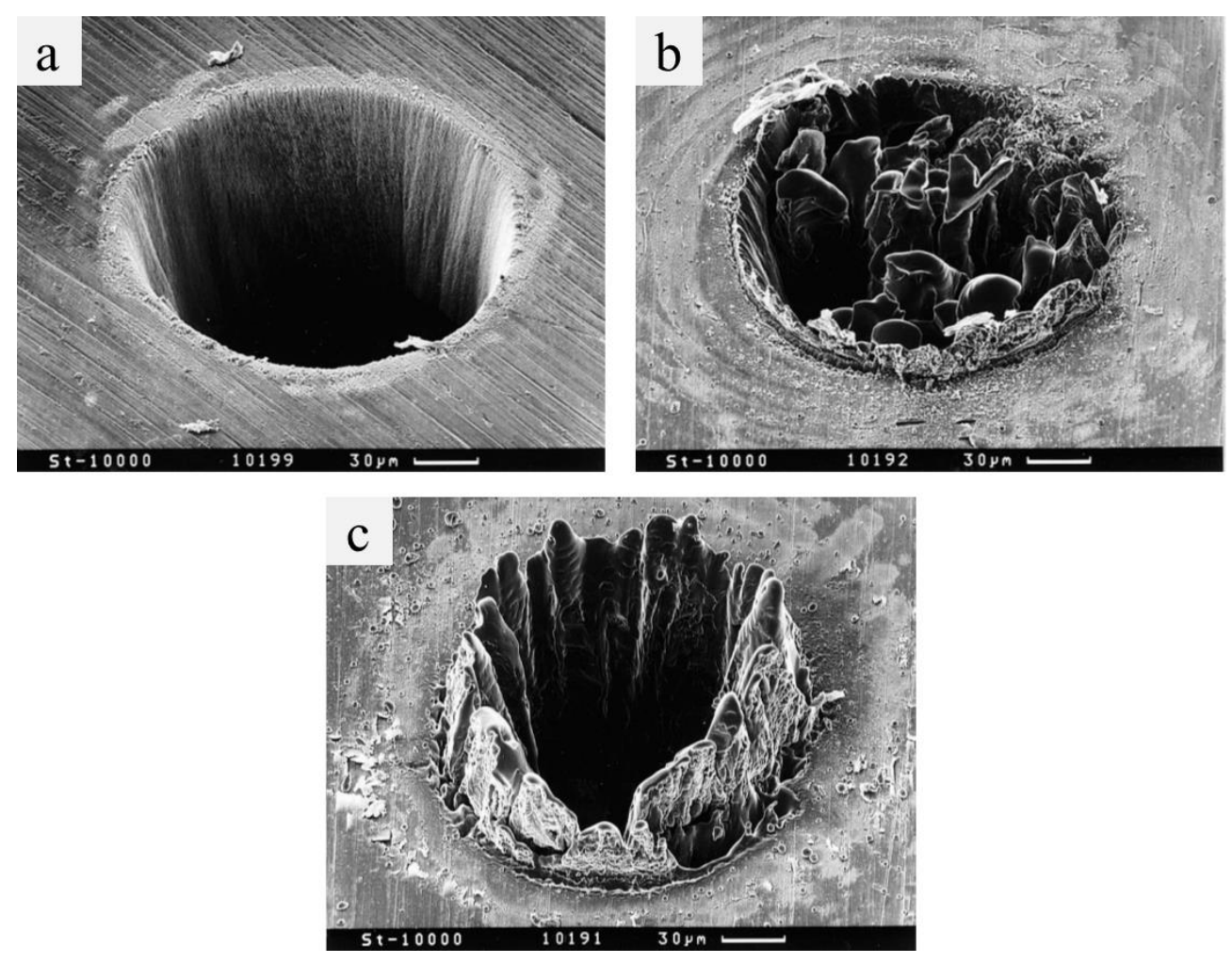

Fig. 1.1. SEM image of holes drilled in a $100 \mu \mathrm{m}$ thick steel foil with (a) $200 \mathrm{fs}, 120 \mu \mathrm{J}, F=0.5 \mathrm{~J} / \mathrm{cm}^{2}$, (b) $80 \mathrm{ps}, 900 \mu \mathrm{J}, F=3.7 \mathrm{~J} / \mathrm{cm}^{2}$, and (c) $3.3 \mathrm{~ns}, 1 \mathrm{~mJ}$, and $F=4.2 \mathrm{~J} / \mathrm{cm}^{2}$. All the three cases are irradiated by laser pulses at wavelength of $780 \mathrm{~nm}$. Figures are from Ref. [28]. 


\subsection{Experimental methods of laser material modification}

A wide spectrum of traditional methods has been developed for surface modification in order to control optical, mechanical, wetting, chemical, and other material properties. Immediately after the introduction of the first ruby laser by Maiman in 1960 [9-11], laser systems have been rapidly introduced in different material applications such as cutting, welding, drilling, etc. due to the laser's ability to process most types of material.

Laser processing techniques have been gradually developed with the aim of tailoring surface properties of the material. These techniques include mask projection technique [29], near-field ablation [34-37], laser assisted chemical etching [38], nanotexturing by deposition from a femtosecond laser ablation plume, [33, 39-42], direct femtosecond laser ablation [33, 43, 44], nanostructuring of thin metal films by femtosecond laser-induced melt dynamics [45, 46], plasmonic nanoablation [47], and interferometric femtosecond laser ablation [19, 48-51]. The generation of laser induced periodic surface structures (LIPSSs), which leads to the formation of periodic nano-grooves, is also considered to be one of the important applications in the area of nanoscale surface modification $[52,53]$.

In order to elucidate the fundamental physics of the surface nanostructuring, the laserinduced formation of nanostructures has been investigated in different environments, like vacuum [54], background gas [39], or liquid [55], as well as under conditions of spatial confinement by a transparent overlayer [56]. One of the numerous examples that can be addressed here is the work by Stratakis et al [55] where the formation of nanostructures by short pulse laser ablation of Aluminum (Al) in liquids, water, and ethanol was investigated. Although, they noticed that the periodic nanostructure does not directly depend on the laser parameters such as the wavelength and the pulse duration, the formation of this nanostructure results in the improvement of the $\mathrm{Al}$ absorption in the UV-vis range. In another study, Klein-Wiele and Simon investigated the effect of the PMMA confinement layer on the quality of laser processing of $\mathrm{Ni}$ and Si by 500 fs laser pulses [57]. The results illustrate that splashing and ejection of the material from the surface is remarkably reduced in the presence of the confinement layer, which results in the improvement of the quality of surface nanostructuring. 
With the rapid development of laser systems and the complexity of the material response to the laser energy deposition, the need for computer modeling is increasing due to its ability to clarify difficult questions which are generated through theory and experiment.

\subsection{Computational modeling of laser-metals interactions}

Computational modeling techniques are used to promote the theoretical understanding of the laser-induced processes and facilitate the advancement of laser applications. We can classify the computational modeling of laser-induced processes into three main categories: the quantum mechanics based ( $a b$ initio) electronic structure calculations [58], the classical molecular dynamics (MD) simulations [59, 60], and the continuum-level kinetic and hydrodynamic modeling [59]. Each one of these categories is operating at specific length and time scales. For instance, the electronic structure calculations are capable of reproducing the initial material response to the laser excitation, including transient changes in interatomic bonding and corresponding modifications of material properties [61, 62], non-thermal structural transformations $[63,64,65]$, bond-breaking/rearrangement and atomic ejection under conditions of strong electronic excitation [66, 67, 68], as well as relaxation of excited states and electronphonon equilibration [69, 70]. On the other hand, the hydrodynamic (HD) modeling adopts multi-phase equations of state to describe the evolution of thermodynamic parameters of the material. The HD modeling is used to draw a clear physical understanding of two specific characteristics: the mechanisms of short pulse laser interactions with material and the dynamics of the material ejection from the target [71-76]. Accordingly, the HD modeling has been used in several areas, which include: investigation of the mechanisms of short pulse laser interaction with metal nanoparticles in the ambient liquid [76, 77], the study of the bubble formation and shock wave generated in the melanosome system while immersed in liquid ambient and irradiated by a laser pulse [78], analysis of the heterogeneous and homogeneous melting mechanisms under the non-equilibrium condition induced by fast laser energy deposition [79], and investigation of the physical processes that lead to the plasma formation in short pulse laser interaction with solid material $[72,80]$.

With the introduction of the multiscale modeling, that combines two or more different length and time scales, the predicted power of the computational modeling have been further increased, making it possible to address the most complex problem. Below we will give a brief 
introduction to the important modeling techniques that are commonly used in the area of shortpulse laser processing, and then will draw specific attention to the models that are used in our studies.

\subsubsection{Continuum modeling}

The irradiation of metal target by short pulse laser (fs up to $10 \mathrm{ps}$ ) has the ability to bring material to a state of strong electronic, thermal, and mechanical non-equilibrium and trigger coupled transient processes that include non-equilibrium energy transport, electron-phonon thermalization, as well as fast non-equilibrium structural and phase transformations that arise from the extreme and dynamic pressure-temperature conditions [59, 81]. Generally, with the deposition of the laser energy in the surface region of the irradiated metal targets, the conduction band electrons can be selectively excited. The electrons are thermalized rapidly and then cooled by slowly transferring their energy to the atomic vibration via the electron-phonon scattering. The latter process is governed by the strength of the electron-phonon coupling that takes from just a few to tens of picoseconds; ultimately, a thermal equilibrium is established between the electron and the lattice.

It has been reported that there are great differences in the laser processing conditions in short pulse laser (fs up to $10 \mathrm{ps}$ ) and long pulse laser (several tens of ps, ns) material interaction. For the long pulse, when the pulse duration is longer than the time required for transferring the energy from the excited electrons to the phonons, the electrons and phonons remain in thermal equilibrium during the laser energy deposition. Consequently, the flow of the heat from the surface region into the bulk of the irradiated target can be described by a heat conduction equation formulated for a single temperature, i.e., the common temperature of the electrons and phonons [82-85]:

$$
C \frac{\partial T}{\partial t}=K \nabla^{2} T(z, t)+S(z, t)
$$

where $C$ is the heat capacity, $K$ is the thermal conductivity, and $S(z, t)$ represents the energy deposition by the laser pulse.

On the contrary, three stages can be identified in the interactions of short pulse laser with metals before the metal returns to its equilibrium state, as illustrated in Fig. 2.1. The first stage is initiated immediately after the absorption of the laser light, which results in thermal excitation of 
the lower band electrons to levels above the Fermi level. The excitation process has a direct effect on the thermophysical properties of the material and it is quite sensitive to the material's electronic structure. For example, in noble metals such as Silver (Ag), Gold (Au), and Copper $(\mathrm{Cu})$, the d-bands electrons are located $\sim 2$ to $3 \mathrm{eV}$ below the Fermi level. After the optical excitation, the excited electrons can penetrate ballistically deeper ( 100-150 nm) into the bulk metal with a velocity close to the Fermi velocity $\sim 10^{6} \mathrm{~m} / \mathrm{s}[86,87]$. In contrast, the Fermi level cuts through the d-band of the transition metals such as Nickel $(\mathrm{Ni})$, and Platinum $(\mathrm{Pt})$, which have almost full d-bands. The combined effect of the laser excitation with the position of the Fermi level in the transition metals creates a high density of electron states at the Fermi level and causes a strong electron-electron interaction, which limits the ballistic motion of the electrons to a range comparable to the optical penetration depth $(\sim 10-20 \mathrm{~nm})[87,88,89]$.

The second stage of equilibration of the electronically-excited material begins after the thermal equilibrium state is achieved among the hot excited electrons. This stage is characterized by the Fermi-Dirac distribution of the electrons with a well-defined high electron temperature $T_{e}$. The electron temperature in such a case can reach tens of thousands of Kelvins, while the lattice temperature still remains within hundreds of Kelvins. This state of strong non-equilibrium between the electron and the lattice temperature can be characterized as a state with two coupled subsystems. The hot electrons are initially localized either within the ballistic range or the optical penetration depth. Accordingly, the hot electrons diffuse deeper into the bulk of the metal target driven by the electron temperature gradient with a diffusion length that is determined by the electron-phonon coupling. During the diffusion process, the energy transfers from the excited electrons to the phonons due to the electron-phonon scattering process. In noble metals, the weak electron-phonon coupling results in a relatively large electron diffusion length and slow the thermal relaxation process, which is in contrast to the transition metals where the thermal relaxation process is faster because of the strong electron-phonon coupling and the shorter length of the electron diffusion. Typically, the thermal equilibration between the electrons and phonons can take anywhere from a fraction to several tens of picoseconds. Finally, the third stage is launched, when the thermal equilibrium between the two subsystems is achieved and, gradually, the whole system returns back to its initial state before the laser excitation. 

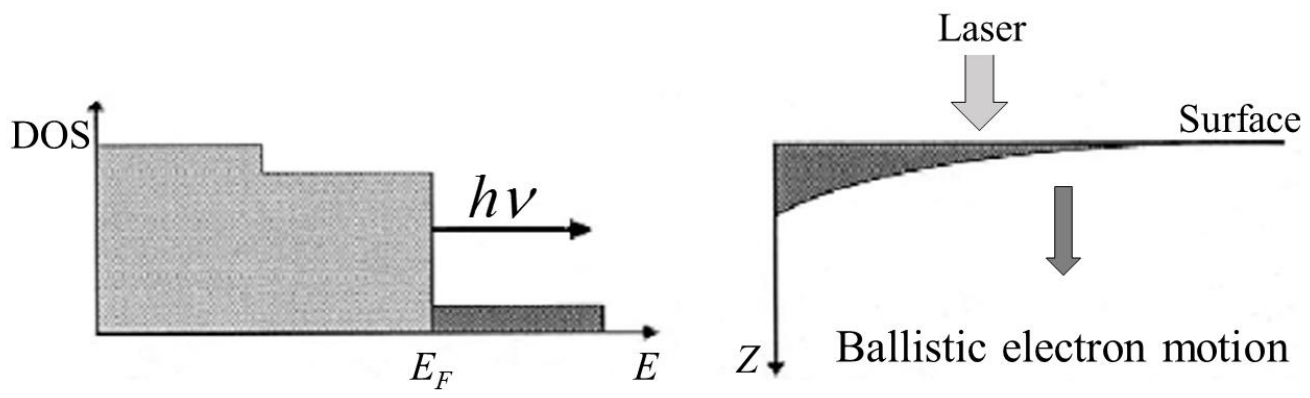

(a) Initial stage : at $t=0$, laser light is absorbed by the conduction band electrons resulting in creating a non-equilibrium state between the electrons. The electrons are moving ballistically with velocity close to Fermi velocity, $v_{F}=\left(\frac{\hbar}{m}\right) k_{F} \approx 10^{6} \mathrm{~m} / \mathrm{s}$.
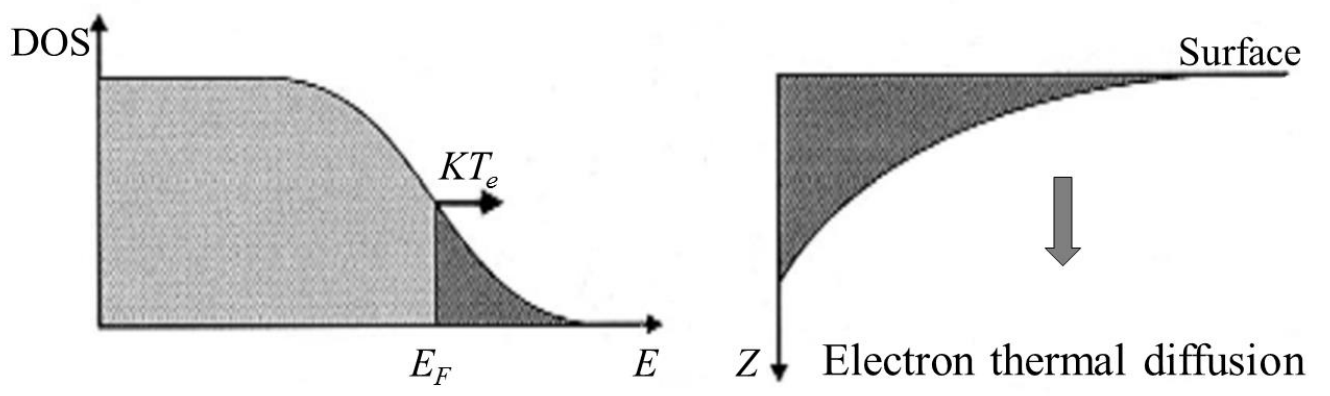

(b) Second stage: this stage is characterized by Fermi-Dirac distribution of the electrons, where a thermal equilibrium state is achieved among the electrons. The electron temperature is much higher than the lattice temperature, $T_{e}>T_{l}$. The electrons diffuse deeper inside the bulk of the metal target driven by the temperature gradient.
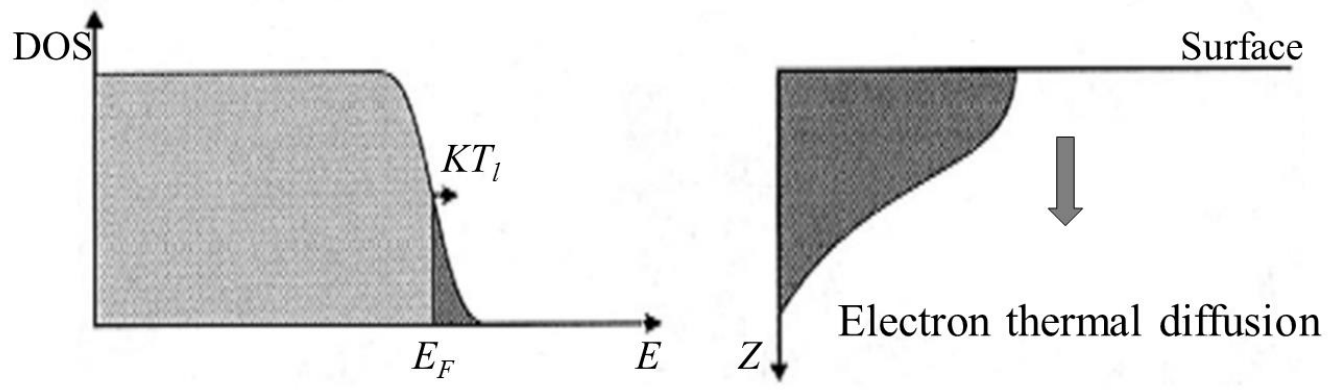

(c) Final stage: a thermal equilibrium state between the electrons and phonons is established via the electron-phonon coupling. Gradually, the Fermi distribution narrows until the whole system returns to its original state before laser excitation.

Fig. 1.2. Three distinct relaxation phases of optically excited electrons in metals. The energy distributions inside the material are shown on the right. The schematic drawing is based on the discussion of Ref. [87]. 
In 1974, Anisimov et al. proposed a relatively simple model to describe the equilibration between the temperatures of the electrons and the lattice that is associated with the second stage of equilibration of the excited electrons discussed above [90]. The model dubbed two temperature model (TTM) describes the evolution of the electron and the lattice temperatures by two coupled of nonlinear differential equations:

$$
\begin{aligned}
& C_{e}\left(T_{e}\right) \frac{\partial T_{e}}{\partial t}=\nabla \cdot\left[K_{e}\left(T_{e}\right) \nabla T_{e}\right]-G\left(T_{e}\right)\left(T_{e}-T_{l}\right)+S(z, t), \\
& C_{l}\left(T_{l}\right) \frac{\partial T_{l}}{\partial t}=\nabla \cdot\left[K_{l}\left(T_{l}\right) \nabla T_{l}\right]+G\left(T_{e}\right)\left(T_{e}-T_{l}\right),
\end{aligned}
$$

where $C_{e}, C_{l}$, represent the heat capacity, $K_{e}, K_{l}$ represent the thermal conductivity for the electrons and the lattice, respectively, $G$ is the electron-phonon coupling factor, and $S(z, t)$ represents the spatial and temporal energy deposition by the laser pulse. Eq. (2) describes the evolution of the electron temperature due to diffusion, coupling to the lattice, and the laser irradiation. In Eq. (3), the evolution of the lattice temperature is represented, and since the heat diffusion is much more rapid in the electrons than the lattice, the lattice heat conductivity (the first term on the right side of Eq. (3)) is normally neglected. The source term $S(z, t)$ that describes the absorbed energy density is defined as:

$$
S(z, t)=I(t)(1-R-T) \frac{\exp \left(-z /\left(L_{0}+L_{\text {ball }}\right)\right)}{\left(L_{0}+L_{\text {ball }}\right)\left(1-\exp \left(-d /\left(L_{0}+L_{\text {ball }}\right)\right)\right)},
$$

where $L_{0}=\alpha^{-1}$ is the optical penetration depth, $L_{\text {ball }}$ is the ballistic length, $d$ is the thickness of the system, $I(t)$ is the variation of the incident intensity of the laser pulse, $R$ and $T$ are the reflectivity and the transmissivity $[87,91]$.

Over the past decades, TTM has become an essential model for analysis of the evolution of the electron and lattice temperatures during the laser-metal interactions. This model has been used to obtain information on thermal conductivity, the electron-phonon coupling [84, 87 , 9294], the mechanical properties of thin films [93], the effect of the grain boundaries [95, 96], and the small size of the system on the electron-phonon relaxation $[97,98]$. The model has also been used to predict the threshold fluence for the laser melting [91], laser induced desorption of 
species from metal surfaces [99], and explain the dynamics of ion ejection in laser ablation [100].

Although, the TTM model has been used successfully in many applications, there are a number of ingrained limitations of this model, which reduces its capability to investigate certain aspects of laser-metal interactions. Some of these limitations can be overcome by adding additional parameters within the framework provided by TTM. In particular, some of the modifications include incorporation of the description of the surface and grain boundary scattering [101], the energy transfer by ballistic electrons [87], combining TTM with the hot electron blast model [102], and thermoelastic equations to investigate the conditions for nonthermal damage to the thin metal films [103]. Other limitations which are hard to overcome using this conventional TTM model are the description of phase transformations occurring under highly non-equilibrium conditions induced in the target material by short pulse laser, the investigation of the generation of crystal defects, the microscopic mechanisms of laser melting, as well as nucleation and growth of voids in photomechanical spallation. Additionally, the characteristics of the explosive volume ablation and the parameters of the ejected multicomponent and multiphase ablation plume cannot be investigated with TTM. All these limitations ingrained in the conventional TTM indicate that this model has to be combined with other computational models in order to provide a realistic representation for the complex material response to the laser energy deposition.

\subsubsection{Atomistic molecular dynamics modeling}

The classical molecular dynamics (MD) simulation is a computational technique that is capable of providing an atomic-level insight into the rapid structural and phase transformations induced by short pulse laser irradiation. The MD simulations initially emerged in 1957 through Alder and Wainwright work, when they used a hard sphere model to describe melting and the dynamic behavior of liquid system consisting of 500 atoms [104]. Gradually, with the revolutionary developments in computer technology and the improvement in the simulation methodologies, MD has become a desirable and commonly used in numerous fields, such as physics, chemistry, biology, and materials science. In a MD simulation, the time evolution of a system of interaction particles (atoms, molecules, granules, etc.) is obtained via the solution of Newton's equations of motion: 


$$
m_{i} \frac{d^{2} \vec{r}_{i}}{d t^{2}}=\vec{F}_{i} \quad i=1,2, \ldots \ldots ., N
$$

where $m_{i}$ and $\vec{r}_{i}$ are the mass and position of a particle $i, \vec{F}_{i}$ is the force acting on $i^{\text {th }}$ particle at a given time. The force can be obtained from the interatomic potential $U\left(\vec{r}_{1}, \vec{r}_{2}, \vec{r}_{3}, \ldots . \vec{r}_{N}\right)$ that is a function of the position of all the particles:

$$
\vec{F}_{i}=-\vec{\nabla}_{i} U\left(\vec{r}_{1}, \vec{r}_{2}, \vec{r}_{3}, \ldots . \vec{r}_{N}\right)
$$

To integrate Eq. (5) for a system consisting of $N$ particles, the initial positions and velocities as well as the instantaneous forces acting on each particle have to be specified. Accordingly, the MD trajectories that describe the time evolution of the system in the phase space can be determine from the position and velocity vectors. Several numerical integration methods have been used in classical MD simulation in order to predict the changes in the positions and velocities of all particles in the system within a finite time interval, such as Euler's Method, Verlet algorithm, and predictor-corrector algorithms [105].

Due to its simplicity and stability, Verlet algorithm is most commonly used in the integration of the equations of motion in MD simulations. The algorithm was first used by Delambre in 1791 and then it had been rediscovered and used by Verlet in 1967 to investigate the equilibrium state for argon system described by two-body interatomic potential [106]. The basic formula for this algorithm can be derived from Taylor's expansion and has the following form:

$$
r_{i}(t+h)=-r_{i}(t-h)+2 r_{i}(t)+\sum_{j \neq i} F\left(r_{i j}(t)\right) h^{2}
$$

where, $h$ represents the time increment. The velocities, which determine the kinetic energy and the temperature in the system, are calculated from the evolving positions of the particles [106]:

$$
v_{i}(t)=\left[r_{i}(t+h)-r_{i}(t-h)\right] / 2 h,
$$

The predictor-corrector algorithms represent another class of methods commonly used in molecular dynamics simulations for the integration of the equations of motion. The importance of these methods lie in: (1) their ability to treat velocity-dependent forces, such as with NoséHoover method for constant temperature simulations, and to provide a good localized conservation of energy, (2) easy adaptation for multiple time step algorithms. These algorithms, 
which were first discussed by Gear in 1966 [107], consist of three main steps: predictor, force calculation, and corrector. Firstly, from the positions and their time derivatives at a given time, one "predicts" the same quantity at time $(t+h)$ according to the Taylor expansion. Secondly, the force acting on a given particle is evaluated at the new predicted positions. Consequently, the differences between the predicted and the calculated accelerations establishes an "error signal". In the final step, the error signal is used to correct the positions, velocities, and higher order time derivations of the positions, with all the corrections proportional to the error signal. The choice of the coefficients in the correction equations mainly depends on the values that lead to optimal stability and accuracy of the MD trajectories [107]. In the molecular dynamics method used in our code, the Nordsieck fifth-order predicted-corrector algorithm is used and the above three steps are implemented as follows:

1- Initially, variables that represent the time derivatives of the positions are defined:

$$
\begin{gathered}
q_{1}(t)=h \frac{d r(t)}{d t}, q_{2}(t)=\frac{h^{2}}{2} \frac{d^{2} r(t)}{d t^{2}}, q_{3}(t)=\frac{h^{3}}{6} \frac{d^{3} r(t)}{d t^{3}}, \\
q_{4}(t)=\frac{h^{4}}{24} \frac{d^{4} r(t)}{d t^{4}}, q_{5}(t)=\frac{h^{5}}{120} \frac{d^{5} r(t)}{d t^{5}}
\end{gathered}
$$

2- The predicted values are calculated (Taylor expansions):

$$
\begin{aligned}
& r(t+h)=r(t)+q_{1}(t)+q_{2}(t)+q_{3}(t)+q_{4}(t)+q_{5}(t) \\
& q_{1}(t+h)=q_{1}(t)+2 q_{2}(t)+3 q_{3}(t)+4 q_{4}(t)+5 q_{5}(t) \\
& q_{2}(t+h)=q_{2}(t)+3 q_{3}(t)+6 q_{4}(t)+15 q_{5}(t) \\
& q_{3}(t+h)=q_{3}(t)+4 q_{4}(t)+10 q_{5}(t) \\
& q_{4}(t+h)=q_{4}(t)+5 q_{5}(t) \\
& q_{5}(t+h)=q_{5}(t)
\end{aligned}
$$

3- Error in acceleration is given by: 


$$
\delta q_{2}(t+h)=\frac{F(t+h)}{2 m} h^{2}-q_{2}(t+h)
$$

4- The correction step is performed:

$$
\begin{array}{ll}
r^{c}(t+h)=r(t+h)+C_{0} \delta q_{2}(t+h) & C_{0}=3 / 20 \\
q_{1}^{c}(t+h)=q_{1}(t+h)+C_{1} \delta q_{2}(t+h) & C_{1}=251 / 360 \\
q_{2}^{c}(t+h)=q_{2}(t+h)+C_{2} \delta q_{2}(t+h) & C_{2}=1 \\
q_{3}^{c}(t+h)=q_{3}(t+h)+C_{3} \delta q_{2}(t+h) & C_{3}=11 / 18 \\
q_{4}^{c}(t+h)=q_{4}(t+h)+C_{4} \delta q_{4}(t+h) & C_{4}=1 / 6 \\
q_{5}^{c}(t+h)=q_{5}(t+h)+C_{5} \delta q_{2}(t+h) & C_{5}=1 / 60
\end{array}
$$

MD method is used widely in simulations of laser-material interactions to provide the microscopic insights into the mechanisms responsible for the melting process under the condition of overheating in a bulk crystal system [108] and systems with free surfaces [109], the generation and propagation of laser induced pressure waves [110,111], and laser ablation [60]. Despite the demonstrated predictive power of the MD method, there are still several limitations of this technique, such as: (1) the short time and length scales that can be treated in the simulations, (2) the absence of explicit representation of electrons, and (3) the lack of quantum effects, all are reported to be the most severe problems in MD simulations. For instance, the electrons are not explicitly represented in the classical MD method and are introduced through the potential energy surface which is a function of the atomic position only, which in turn, is approximated by an analytical function that gives the potential energy as a function of the coordinates, i.e. $U\left(\vec{r}_{1}, \vec{r}_{2}, \vec{r}_{3}, \ldots . \vec{r}_{N}\right)$ in Eq. (6). This representation of the electrons in MD method results in a nonphysical representation of some of the important processes in the area of laser-metal interactions such as the electron contribution to the thermal conductivity of the metals, the laser energy absorption by the conduction band electrons, and the transient state of the electron-lattice nonequilibrium [112-114]. In order to achieve a more realistic description of laser-induced processes, the advantages of the MD method and the TTM model are combined together in a hybrid atomistic-continuum model. In the next section, we are going to describe in more detail the hybrid computational model that is used in the simulations reported in this dissertation. 


\subsubsection{Combined continuum-atomistic modeling}

As discussed above, the TTM model provides an adequate description of the laser energy absorption by the conduction band electrons, the energy exchange between the excited electrons and phonons, and the electron contribution to the thermal conductivity. The MD method, on the other hand, is capable of modeling laser melting, explosive homogenous boiling, material ejection and ablation, and plume formation. Together, the advantages of the two methods can be combined to overcome the individual limitations of TTM and MD method. Accordingly, a hybrid atomistic-continuum (TTM-MD) model has been developed in order to accurately describe the diverse range of processes induced by short pulse laser irradiation of a metal target [115-121].

Within the TTM-MD model, the computational system consists of two parts, the atomistic TTM-MD part and the continuum TTM part as illustrated in Fig. 1.3. In the TTM-MD part, the uppermost surface region of the metal target, where the fast non-equilibrium processes of lattice superheating, melting, photomechanical spallation, and phase explosion take place, is described by MD method. The MD method fully substitutes the TTM equation for the lattice temperature. In this part, the cells in the finite difference discretization used for the numerical solution of the TTM equation for the electron temperature are related to the corresponding volumes of the MD system. The average kinetic energy of the thermal motion of the atoms, $K^{T}$, is used to calculate in the local lattice temperature, Eq. (14). Furthermore, the conventional MD equations of motion are modified by adding an extra term to account for the electron-phonon coupling:

$$
\begin{aligned}
& m_{i} \frac{d^{2} \vec{r}_{i}}{d t^{2}}=\vec{F}_{i}+\xi m_{i} v_{i}^{T} \\
& T_{l}^{\text {Cell }}=\frac{2 K^{T}}{3 k_{B}}=\sum_{i=1}^{N^{\text {cell }}} m_{i}\left(v_{i}^{T}\right)^{2} /\left(3 k_{B} N^{\text {cell }}\right) \\
& \xi=\frac{1}{n} \sum_{K=1}^{n} G V_{N}\left(T_{e}^{K}-T_{l}\right) / \sum_{i} m_{i}\left(v_{i}^{T}\right)^{2}=\frac{G V_{N}\left(T_{e}-T_{l}\right)}{2 K^{T}}
\end{aligned}
$$


In the term $\xi m_{i} v_{i}^{T}$, the thermal velocities of the atoms are distinguished from the velocities of the collective motion and one can define the thermal velocity for any atom as $v_{i}^{T}=v_{i}-v^{C}$, where $v_{i}$ is the actual velocity of an atom $i$ and $v^{C}$ is the velocity of the center of mass of the cell where the atom $i$ is located. The extra term in the MD equations of motion is employed to play the same role as does the coupling term $G\left(T_{e}-T_{l}\right)$ in the equation for the lattice temperature in TTM, Eq.(2) in Fig. 1.3. Consequently, the coupling terms in both equations (Eq.2 and Eq.15) should be consistent with each other.

In the second part, the TTM part, the diffusion equation for the electron and lattice temperatures is solved in a much deeper region of the target that is affected by thermal conduction (Eq.1 and Eq.2 in Fig. 1.3). The size of the TTM part is determined so that there are no substantial changes in the electron and lattice temperatures in the lowest region of the computational system by the end of the simulation.

The time steps in the two parts of the computational system are defined in a way that one MD time step is equivalent to an integer number of time steps in the finite difference integration of the TTM equation for the electron temperature, $\Delta t_{M D}=n \Delta t_{F D}$, where the $\Delta t_{F D}$ is estimated based on the von Neumann stability criterion, $\Delta t_{F D} \leq 0.5\left(\Delta x_{F D}\right)^{2} C_{e}\left(T_{e}\right) / K_{e}\left(T_{e}, T_{l}\right)$ and $\Delta x_{F D}$ is the spatial discretization step in the finite difference integration.

This difference in the time steps can be directly related to the electron temperature: in one MD time step, the temperature of the electron may drastically change while the lattice temperature stays approximately the same. The lattice temperature, $T_{l}$, is obtained from Eq. (14) and then enters the coupling coefficient $\xi$, Eq. (15), that is recalculated at each MD integration time step for each finite difference discretization cell. Therefore, the equations of motion depend on the changes of the local electron and lattice temperatures. In each finite difference discretization cell, the exchange of energy between the electrons and the lattice via the electronphonon coupling, $\Delta E^{e-p h}=\sum_{K=1}^{n} \Delta t_{F D} G V_{N}\left(T_{e}^{K}-T_{l}\right)$, is collected for a number of integration steps. Then, it is transferred to/extracted from the energy of the atomic motion in the corresponding part of the MD system through the coupling term added to the MD equations of motion. 


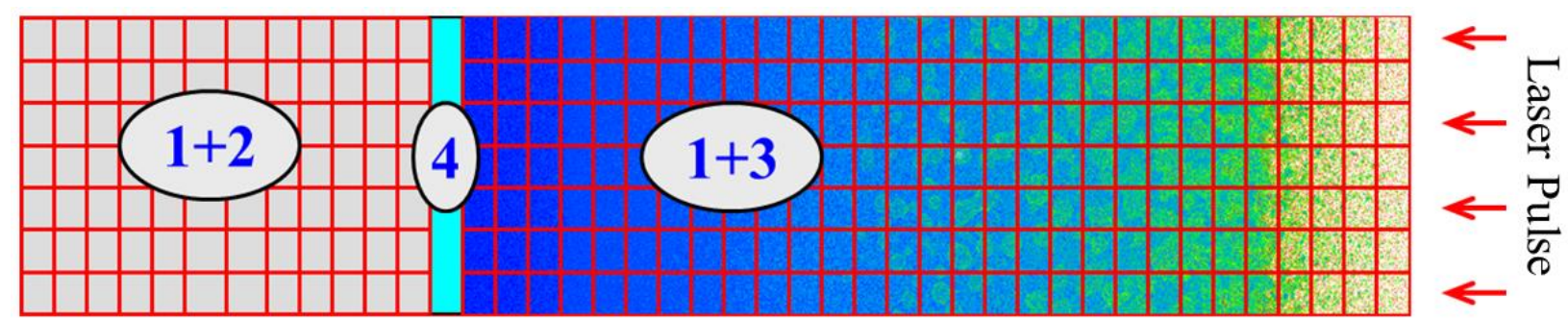

TTM:

$1-\quad C_{e}\left(T_{e}\right) \frac{\partial T_{e}}{\partial t}=\nabla \cdot\left[K_{e}\left(T_{e}\right) \nabla T_{e}\right]-G\left(T_{e}\right)\left(T_{e}-T_{l}\right)+S(z, t)$

2- $C_{l}\left(T_{l}\right) \frac{\partial T_{l}}{\partial t}=\nabla \cdot\left[K_{l}\left(T_{l}\right) \nabla T_{l}\right]+G\left(T_{e}-T_{l}\right)$

\section{TTM-MD:}

3- $m_{i} \frac{d^{2} \vec{r}_{i}}{d t^{2}}=\vec{F}_{i}+\xi m_{i} v_{i}^{T}, T_{l}^{\text {Cell }}=\frac{2 K^{T}}{3 k_{B}}=\sum_{i=1}^{N^{\text {cell }}} m_{i}\left(v_{i}^{T}\right)^{2} /\left(3 k_{B} N^{\text {cell }}\right)$

4- Pressure-transmitting, heating-conducting boundary conditions

Fig. 1.3. Schematic of the TTM-MD model for simulation of laser interaction with metal targets. The laser irradiation is directed from the right side of the figure and periodic boundary conditions are used in the lateral (parallel to the irradiated surface) direction. Spatial discretization in the continuum part of the model and the dimensions of the atomistic and continuum regions are not drawn to the scale. Figure is from Ref. [24].

The irradiation of the computational system by short pulse laser is represented by the source term $S(z, t)$ that describes the local laser energy deposition per unit area and unit time during the laser pulse:

$$
S(z, t)=I_{0}(1-R) L_{P}^{-1} \exp \left(-z / L_{P}\right) \exp \left[-\left(t-t_{0}\right)^{2} / 2 \sigma^{2}\right]
$$

In Eq. (16), $I_{0}$ is the peak intensity, $R$ is the reflectivity, and $\sigma$ is the standard deviation of the Gaussian profile that is related to the pulse duration, $\tau_{l}=\sigma \sqrt{8 \ln (2)}$. The representation of the 
laser fluence, which corresponds to the peak intensity $I_{0}$, can be described as $F=\sqrt{\frac{\pi}{4 \ln (2)}} \tau_{l} I_{0}$. In this model, the absorbed laser fluence is considered and defined as $F_{a b s}=F(1-R)$. According to Ref. [87, 91], and in order to account for the energy transport occurring before the excited electrons equilibrate, the optical penetration depth, $L_{P}$, in Eq. (16) is represented by the summation of the optical absorption depth and the ballistic range, $L_{b}, L_{P}=L_{p}+L_{b}$ where $L_{b}$ can be estimated from the Fermi velocity and Drude electron relaxation time [122], while $L_{p}$ is defined as the reciprocal of the optical absorption coefficient. Due to the small lateral size and the depth of the computational system compared to the laser spot size, a uniform distribution of the laser energy deposition is applied along the $\mathrm{X}$ and $\mathrm{Y}$ axes, and, in addition to that, a onedimensional diffusion equation is solved for the electron and lattice temperatures in the continuum part.

In order to achieve a realistic representation of laser-induced processes by the TTM model, the thermophysical properties of the material (the electron-phonon coupling, the electron heat capacity, and the heat conductivity), which are all affected directly by the thermal excitation of electrons, should be implemented correctly. As discussed in section 1.3.1, the thermophysical properties of metals are influenced by the thermal excitation of the lower band electrons. According to Ref. [86, 123], at higher electron temperature, the strength of the electron-phonon coupling can increase with increasing the electron temperature for the noble metals $\mathrm{Au}, \mathrm{Cu}$, and $\mathrm{Ag}$ ) as well as for $\mathrm{Al}$, with a positive deviation in the electron heat capacity away from the linear dependence of the electron temperature. On the other hand, the transition metals (e.g., Ni, Pt), display an opposite trend represented by the decrease in the electron-phonon coupling and a negative deviation of the electron heat capacity from the linear dependence on electron temperature. The thermophysical properties of Tungsten (W) and Titanium (Ti) show complex dependence on the electronic temperature. The different response to the increase in the electronic temperature is related to the differences in the electronic density of state for different types of metals.

Turning to the electron thermal conductivity, several methods are used to describe the electron temperature dependence of the thermal conductivity in the TTM model [124]. If the electron temperature is significantly lower than the Fermi temperature, the contribution of the electron-electron scattering process is usually neglected as it is much smaller than the one of the 
electron-phonon scattering. In this case, the electron thermal conductivity can be described by $K_{e}=K_{0} \frac{T_{e}}{T_{l}}$. With the increase of the electron temperature, the latter representation of the thermal conductivity becomes invalid. Accordingly, the calculation of the electron thermal conductivity should include both electron-phonon and electron-electron scattering, which can be done within the Drude model [122], $K_{e}=\frac{1}{3} v_{F}^{2} C_{e} \tau_{e}$, where $\tau_{e}$ is the electron relaxation time, $C_{e}$ is the electron heat capacity, and $v_{F}$ is the Fermi velocity. The value of $\tau_{e}$ results from the contribution of the electron-electron scattering time $\tau_{e-e}$ and the electron-phonon scattering time $\tau_{e-p h}, \frac{1}{\tau_{e}}=\frac{1}{\tau_{e-e}}+\frac{1}{\tau_{e-p h}}=A T_{e}^{2}+B T_{l}$. The parameters $A$ and $B$ are the material constants that can be estimated from the free electron model following the procedure suggested in Ref. [125] for $A$, and from the experimental value of the thermal conductivity of the material for $B$ [126]. Subsequently, assuming a linear dependence of the electron heat capacity on the electron temperature, $C_{e}=\gamma T_{e}$, the thermal conductivity is given by:

$$
K_{e}=\frac{1}{3} v_{F}^{2} C_{e} \tau_{e}=\frac{1}{3} v_{F}^{2} \frac{\gamma T_{e}}{A T_{e}^{2}+B T_{l}}
$$

Anisimov and Retherfeld proposed another method for the electron thermal conductivity calculation which is considered to be more suitable for a wide range of temperatures due to its capability of providing an accurate description of the electron heat conductivity at temperatures exceeding the Fermi temperature, in the state of low-density plasma [127]:

$$
K_{e}=C \vartheta_{e} \frac{\left(\vartheta_{e}^{2}+0.16\right)^{5 / 4}\left(\vartheta_{e}^{2}+0.44\right)}{\left(\vartheta_{e}^{2}+0.092\right)^{1 / 2}\left(\vartheta_{e}^{2}+b \vartheta_{l}\right)}
$$

In the above equation, $\vartheta_{e}$ and $\vartheta_{l}$ are electron and lattice parameters that depend on the electron, lattice, and the Fermi temperature.

In the TTM-MD part of the computational system, the interatomic interactions are defined by the embedded atom method (EAM) potential that describes the thermal and elastic properties of the material. The fundamental idea of the EAM potential is based on the densityfunctional theory where the electron density in the vicinity of each atom is determined by the 
aggregate of the contributions to the electron density at the location of the atom from all the surrounding atoms. The total energy of a system of interacting atoms can then be expressed as follows:

$$
\begin{aligned}
& E_{t o t}=\sum_{i} E_{i} \\
& \rho_{i}=\sum_{i \neq j} f_{i}\left(r_{i j}\right) .
\end{aligned}
$$

Eqs. (19) and (20) represent the total internal energy, $E_{t o t}$, that is equal to the sum of the energies of individual atoms $E_{i}$. For each atom $i$, the energy $E_{i}$ is defined by a contribution from the energy required to embed the atom in an electron density $\rho_{i}, F\left(\rho_{i}\right)$, plus the contribution of a two-body potential $\phi_{i j}$ between atom $i$ and atom $j$, separated by distance $r_{i j}$. The ability of the EAM potential to describe the variation of bond strength with coordination makes it more realistic in description of metals as compared to pair potentials.

In our model, the functional form and the parameterizations of $\mathrm{Cu}, \mathrm{Ag}, \mathrm{Au}, \mathrm{Ni}, \mathrm{Pd}$, and $\mathrm{Pt}$ metals are implemented in the form suggested by Foiles, Baskes, and Daw, “EAM-FBD” [128]. A cut-off function is added to the potential in order to bring the interaction energies and forces smoothly to zero at the interatomic distance of $5.5 \AA$. This " $E A M-F B D$ ” potential is fitted to lowtemperature values of the equilibrium lattice constant, sublimation energy, bulk modulus, elastic constants, and vacancy formation energy. Simultaneously, the potential provides a good description of high temperature thermodynamic properties of the metals as relevant to the conditions of the laser-induced melting and ablation. Other parameterizations of the EAM potential have been used for the Cr system according to a modification of the "EAM-Johnson" potential [129]. The $\mathrm{Cr}$ parameters are fitted to the experimental equilibrium lattice constant, cohesive energy, vacancy formation energy, bulk modulus, Voigt average shear modulus, and anisotropy ratio $C_{44} / C^{\prime}$. In the anisotropy ratio, the $\mathrm{C}_{44}$ and $C^{\prime}=\left(C_{11}-C_{12}\right) / 2$ are the two shear moduli of the cubic crystal [129]. Within this potential, no explicit cut-off function is added since the pair potential and the electron density function vanish beyond the third neighbor shell in the $\mathrm{Cr}$ bcc structure, i.e. the contribution from the fourth neighbors to the interaction energy is two orders of magnitude smaller than that from the third neighbors. 
Lastly, different types of boundary conditions are implemented in the computational system. Periodic boundary conditions are imposed in the lateral directions, parallel to the surface of the computational system. The large diameter of the laser spot $(\sim 10-100 \mu \mathrm{m})$ as compared to the depth of the sample affected by laser-induced phase transformations ( 100-500 nm) makes it possible to neglect the lateral variation of the laser intensity and represent the interaction of the atoms in the MD computational cell with the surrounding material through the periodic boundary conditions. A free boundary condition is applied in the direction normal to the irradiated surface in order to simulate the uniaxial expansion as well as the ejection of vapor atoms or liquid droplets during the ablation process. To preclude the reflection of the laser-induced pressure wave from the bottom of the MD computational cell and to ensure energy transfer to the bulk of the target, a non-reflecting boundary condition (NRB) is implemented at the boundary between the atomistic TTM-MD and the continuum TTM parts of the model, region 4 in Fig. 1.3. The atoms in the NRB region experience a combination of three types of forces: (1) $F_{0}$, the static force that acts between the atoms even if the atoms are at rest, (2) $F_{W}$, the force due to the propagation of the pressure wave through the boundary region, and (3) $F_{L}$, the force due to the direct laser energy deposition in the boundary region on the timescale shorter than the one of the mechanical relaxation $[110,130]$. For the third type of forces $\left(F_{L}\right)$, in order to account for the laser-induced pressure relaxation, a damping factor $D=L_{P}\left(1-\exp \left(t c / L_{P}\right)\right) / t c$ is introduced, where $L_{P}$ is the characteristic size of the region heated by the laser energy deposition, $C$ is the velocity of the pressure wave, and $t$ is the simulation time [110].

The combination of the advantages of the TTM and the MD method enables an adequate description of the laser energy absorption by the electronic system, the energy exchange between the electrons and phonons and the fast electron heat conduction in metals. Simultaneously, the hybrid computational model allows for investigation of the non-equilibrium processes of lattice superheating, melting and ablation.

\subsection{Objectives of this study}

Modification of surface properties by short pulse laser is attracting worldwide interest within the industry and in the scientific research community. This is directly related to the unique capability of a short pulse laser to provide high precision control over surface modification with 
minimal collateral damage, along with its ability to ablate any material with a large material removal rate. Consequently, material processing by short laser pulses is used in many current and emerging applications in the areas of photonics, plasmonic, optoelectronics, biochemistry, etc. [131,132]. The interaction of short pulse lasers with metals induces a range of interrelated processes, such as melting and solidification, generation of crystal defects, fracture and disintegration of the region of the laser energy deposition due to the photomechanical stress (front surface spallation), vaporization and explosive boiling of strongly overheated surface region, ionization and plasma formation [133].

The necessity for computer modelling increases with the development of both new laser systems and surface modification processes. The application of computer modeling provides a deeper theoretical understanding of the laser-induced processes and helps in the advancement of laser applications [24, 60, 134]. Accordingly, the objective of this study is to provide a solid understanding of the mechanisms responsible for short pulse interaction with metals by performing a detailed computational analysis of laser-induced processes in several representative metals $(\mathrm{Cr}, \mathrm{Ni}$, and $\mathrm{Ag}$ ) irradiated by femtosecond and picosecond laser pulses. Below is a list of the main objectives of this study that are reported in this dissertation.

\section{Molecular dynamics study of femtosecond laser pulse interactions with $\mathrm{Cr}$ targets}

a. In the first part of this study, a series of TTM-MD simulations of bulk bec Cr targets irradiated by 200 fs laser pulses at a broad range of absorbed laser fluences, from 430 to 3830 $\mathrm{J} / \mathrm{m}^{2}$ are performed. The absorbed laser fluences cover three distinct regimes, laser melting and solidification of the surface region of the target, photomechanical spallation of liquid layers, and phase explosion and ejection of a superheated surface region. The transitions between different regimes are identified according to the total amount of ejected material as well as the composition of the ablation plume. The results obtained in these simulations are presented in chapter 2, sections 2.3.1 and 2.3.2, and reported in Ref. [134].

b. In order to provide a realistic representation of the laser-induced photomechanical spallation, a large scale simulation of femtosecond laser pulse interactions with (001) Cr target is performed. The results reveal the microscopic mechanisms responsible for the photomechanical spallation of a liquid layer from the surface region of the target. The relaxation of the strong compressive stresses generated by the rapid laser energy deposition leads to the emergence of an 
unloading tensile wave, which in this simulation exceeds the dynamic strength of the material resulting in nucleation, growth, coalescence, and percolation of sub-surface voids. The evolution of the voids leads to a complete spallation of a molten layer from the target and generation of complex surface morphology after complete resolidification of the target. The characteristic features of the surface morphology predicted in the simulation are found to match these absorbed in laser processing experiments. The results obtained in this simulation are presented in chapter 2, section 2.3.3.

\section{Generation of crystal defects in different regimes of laser interactions with metals}

a. The detailed analysis of the surface region of $\mathrm{Cr}$ target for all the simulations discussed in section 2.3.2 reveals the formation of high density of point defects (mostly vacancies, but also some interstitials) in the region that experienced transient melting and resolidification. The generation of the large number of vacancy may have important implications on the physical, chemical, and mechanical properties of the surface layers. The variation of the vacancy concentration with depth under the irradiated surface is analyzed for each simulation and related to the normalized surface temperature at the end of the solidification process. The concentration of vacancies is also determined in bulk $\mathrm{Ni}$ with fcc crystal structure, in order to compare the results with the ones obtained for bulk bcc $\mathrm{Cr}$ targets. The analysis of the concentration of vacancies is carried out for earlier results of $\mathrm{Ni}$ target irradiated by a 1 ps laser pulse at a range of absorbed laser fluences covering the same three irradiation regimes as in the simulations for $\mathrm{Cr}$. The results of this part of the computational study are presented in chapter 3 , section 3.3.

b. The mechanisms responsible for the generation of linear defects, dislocations, and the dependence of the dislocation emission on the crystallographic orientation of the irradiated targets are investigated in large-scale parallel TTM-MD simulations of $\mathrm{Cr}$ targets with (001), (110), and (111) surfaces. The three targets are irradiated by a $50 \mathrm{fs}$ laser pulse at an absorbed laser fluence of $800 \mathrm{~J} / \mathrm{m}^{2}$, which is above the threshold for laser melting and below the threshold of spallation. The detailed analysis of the results obtained from the three simulations reveals the formation of unstable partial dislocations (island of stacking faults) in (001) $\mathrm{Cr}$ target, and a high density of perfect dislocations in (110) and (111) Cr targets. Furthermore, the formation of high density of point defects in the surface region of the targets is observed in all three simulations. The details are presented in chapter 3, section 3.4. 


\section{Generation of point defects by rapid solidification of $\mathrm{Ni}$ and $\mathrm{Cr}$ under conditions of strong undercooling}

a. A series of $\mathrm{MD}$ simulations of the solidification process in $\mathrm{Cr}$ and $\mathrm{Ni}$ systems is performed under well-controlled temperature and pressure conditions in order to identify the mechanisms responsible for the vacancies formation. The distribution of the vacancies is analyzed in each atomic plane and related to the velocity of the solidification front. Close up investigation of the interface region is also undertaken to determine the dominant mechanism responsible for the vacancy formation. The vacancies diffusivity as well as the time between the vacancy jumps are determined for each system. The simulations reveal that the dominant mechanism responsible for the vacancies formation in both systems is the generation of vacant lattice sites at the rapidly advancing solidification front during the solidification process. The results of the simulations are presented in chapter 4 , section 4.3.

b. To study the effect of homogeneous nucleation of new crystallites in the supercooled liquid on the velocity of solidification front, another series of MD simulations is performed where the interatomic interaction in the Ni system is described by the embedded atom method potential in the form proposed by Foiles, Baskes, and Daw, "EAM-FBD". In total, 52 simulations of EAM-FBD $\mathrm{Ni}$ system are performed at different specific undercooling temperatures and constant zero pressure. The temperature variation is from $0.95 T_{m}$ to $0.1 T_{m}$ with temperature step equal to 0.02 . The results obtained from these simulations are compared to the one obtained from the series of MD simulations for $\mathrm{Ni}$ system where the interatomic interactions are described by "EAM-Johnson" potential. In both cases, the curve of solidification front velocity with respect to the normalized temperature shows a linear dependence in the high temperature regime, from temperature close to the melting temperature down to $0.7 T_{m}$. This behavior change in low temperature regime. While the velocity curve for the EAM-FBD Ni decreases with the reduction of temperature, the velocity predicted for EAM-Johnson Ni remains constant down to a temperature of $0.1 T_{\mathrm{m}}$. The results for EAM-FBD Ni illustrate the nucleation of crystalline structures inside the liquid phase that grow and impact the advancement of the solidification front. The results of this part of the study are presented in chapter 4, section 4.4. 


\section{The effect of spatial confinement by transparent overlayer on the laser melting, photomechanical spallation, and phase explosion of metal targets irradiated by short laser pulse}

a. The general mechanisms of laser melting, spallation, and ablation of metals in vacuum has been extensively studied experimentally, theoretically, and computationally. On the other hand, the effect of strong spatial confinement by a solid transparent overlayer on the laserinduced processes remains largely unexplored. Accordingly, two series of large-scale atomistic simulations are performed for a Ag target, one of them carried out under a vacuum condition and another for a target covered by a transparent overlayer. The material response to the laser energy deposition is studied for a range of laser fluences that, in the absence of the transparent overlayer, covers the regimes of melting and resolidification, photomechanical spallation, and phase explosion of the overheated surface region. In contrast to the irradiation in vacuum, the spatial confinement by the overlayer facilitates generation of sustained high-temperature and pressure conditions near the metal-overlayer interface, suppresses the generation of unloading tensile wave, decreases the maximum depth of melting, and prevents the spallation and explosive disintegration of the surface region of the metal target. At high laser fluences, when the laser excitation brings the surface region of the metal target to supercritical conditions, the confinement prevents the expansion and phase decomposition characteristic for the vacuum conditions and leads to a gradual cooling of the hot compressed supercritical fluid down to the liquid phase and eventual solidification. The target modification in this case is limited to the generation of crystal defects and the detachment of the metal target from the overlayer. The results obtained in these simulations are presented in chapter 5, section 5.3, and reported in Ref. [56].

b. The short pulse laser interaction with metal surfaces covered by transparent overlayers is investigated in experiments and atomistic simulations, with a particular aim of revealing the mechanisms responsible for structural modification of the metal-overlayer interfacial regions. Experimental characterization of Al-silica targets modified by single pulse laser irradiation with the pulse duration of $10 \mathrm{ps}$ reveals the transitions from the generation of extended interfacial voids with internal nanoscale surface roughness to the partial detachment of the overlayer from the metal substrate, and to the cracking/chipping or complete removal of the overlayer as the laser fluence increases. The mechanisms responsible for the appearance, growth, and percolation 
of the interfacial voids leading to the detachment of the overlayer from the metal substrate are investigated in a large-scale atomistic simulation. The results of the simulations demonstrate that the processes of nucleation and growth of the interfacial voids are driven by the dynamic relaxation of laser-induced stresses proceeding simultaneously with rapid phase transformations and temperature variation in the interfacial region. The growth and coalescence of the interfacial voids result in the formation of liquid bridges connecting the overlayer and the metal substrate, whereas solidification of the transient liquid structures produced by breakup of the liquid bridges may be responsible for the formation of the nanoscale roughness of the interfacial voids observed in experiments. Computational analysis of the effect of pre-existing interfacial voids reveals a complex dynamic picture of the initial expansion and subsequent compaction of the surface region of the metal substrate and suggests a possible scenario for the formation of voids below the metal-overlayer interface. The experimental and computational results are presented in section 5.2, and reported in Ref. [189]. 


\section{Chapter 2}

\section{Molecular dynamics study of femtosecond laser interactions with $\mathrm{Cr}$ targets}

The results and discussion in sections 2.1, 2.2, 2.3.1, and 2.3.2 are reported in the following paper:

E. T. Karim, Z. Lin, and L. V. Zhigilei, Molecular dynamics study of femtosecond laser interactions with Cr targets, International Symposium on High Power Laser Ablation 2012, AIP Conf. Proc.1464, 280-293 (2012). 


\subsection{Introduction}

The fast advancement of laser technology makes ultrashort (picosecond and femtosecond) lasers increasingly accessible and expands the range of material processing applications that benefit from the rapid laser energy deposition, small heat affected zone, and minimal residual damage characteristic of the ultrashort pulse irradiation $[28,91,135,136]$. The ability of the ultrashort pulses to generate well-defined surface micro/nanostructures with remarkable quality and reproducibility [137-139] and to perform high precision drilling and cutting of a broad range of materials [28, 140, 135] has been demonstrated in numerous studies.

The active expansion of the area of practical applications of ultrashort laser pulses motivates experimental and theoretical studies focused on the fundamental mechanisms of lasermaterials interactions. Under conditions when laser irradiation results in permanent surface modification or material ejection (ablation), the relevant processes discussed in literature are the transient melting and solidification of a thin surface layer [86, 120, 141-143], the explosive boiling (phase explosion) of a surface region superheated beyond the limit of thermodynamic stability of the molten material [23, 114, 120,144-147], the melt expulsion or sputtering due to the action of ablation recoil pressure [120, 148], the ejection of large droplets or fractured solid fragments caused by the relaxation of photomechanical stresses [113, 117, 118, 120, 149-153], as well as non-thermal phase transformations induced by the electronic excitations [154-157], photochemical reactions [158] or charge separation and Coulomb explosion [159].

Molecular dynamics (MD) simulations are playing an increasingly important role in investigations of the complex and highly non-equilibrium processes induced by short pulse laser irradiation, see reviews $[121,160]$. In particular, MD simulations of laser interactions with metal targets have provided important insights into the mechanisms and atomic-level dynamics of laser melting $[118,120,129,141,143,161,162] . s p a l l a t i o n ~[117,118,120,152,153]$ and explosive boiling [120, 146]. While most of the MD simulations of laser interactions with metals have been performed for face-centered-cubic (fcc) metals, recent simulations of laser melting and resolidification of body-centered-cubic (bcc) metal, Cr, has also been reported [129].

In this section, initially we report an extension of the MD simulation study of the femtosecond laser melting of $\mathrm{Cr}$ targets to higher fluences that cover the irradiation regimes of 
photomechanical spallation and explosive boiling. The results of the simulations are related to the ones obtained in a study of laser interactions with fcc Ni targets [120] where strong connections between the maximum melting depth, photomechanical spallation, and phase explosion are established. Then, we provide a microscopic view of the mechanisms of laserinduced photomechanical spallation from a large scale TTM-MD simulation of a bulk Cr target irradiated by a femtosecond laser pulse. The computational models used in these simulations are described next, in Section 2.2. The results of the simulations are presented and discussed in Section 2.3, and summarized in Section 2.4.

\subsection{Computational model}

The simulations of laser interactions with $\mathrm{Cr}$ target are performed with a combined atomistic-continuum model that couples the classical MD method with a continuum-level description of the laser excitation and subsequence relaxation of the conduction-band electrons. This model is described in more details in section 1.3.3.

Two series of simulations, with small and large computational cells, are performed with the aim of investigation of the mechanisms of laser-induced melting and solidification of the surface region of the target, photomechanical spallation, and explosive disintegration and ejection of the superheated surface region. The interatomic interaction in the MD part of the model is represented by the embedded atom method EAM with functional form and parameterization developed for $\mathrm{Cr}$ [129]. The potential provides a computationally efficient yet sufficiently accurate description of real $\mathrm{Cr}$ metal. The equilibrium melting temperature, the enthalpy of melting, heat capacity, and vacancy migration energy of the EAM Cr material are within 10\% of the experimental values. A good agreement of the calculated elastic properties (including the negative sign of the Cauchy pressure characteristic of $\mathrm{Cr}$ ) with experimental data is also obtained [129]. The dimensions of the computational cells and the irradiation conditions used in the two series of simulations are given as follow:

\subsubsection{Computational setup in small-scale simulations}

The MD part of the TTM-MD model represents the top $100 \mathrm{~nm}$ surface region of the target, has lateral dimensions of $8 \times 8 \mathrm{~nm}^{2}$, and is composed of 630000 atoms. Periodic boundary conditions are used in the directions parallel to the (100) free surface of the bcc crystal. To 
ensure non-reflecting propagation of the laser induced pressure wave from the MD part of the model, a dynamic pressure-transmitting boundary condition is applied at the bottom of MD computational cell $[110,130]$. The computational system is equilibrated at $300 \mathrm{~K}$ before applying laser irradiation.

In the continuum part of the model, beyond the MD region, the electron heat conduction and the energy exchange between the electrons and the lattice are described by the conventional TTM. The size of the continuum (TTM) region is varied between $500 \mathrm{~nm}$ and $1.5 \mu \mathrm{m}$ depending on the laser fluence and the duration of the simulation, so that no substantial changes in the electron and lattice temperatures are observed at the bottom of the continuum region by the end of the simulation.

The choice of the parameters used in the TTM equation for the electron temperature is explained in Ref. [129] and the parameters are listed below. The electronic heat capacity is $C_{e}=\gamma T_{e}$ with $\gamma=194 \mathrm{Jm}^{-3} \mathrm{~K}^{-2}$, the electron-phonon coupling constant is equal to $G=4.2 \times 10^{17}$

$\mathrm{Wm}^{-3} \mathrm{~K}^{-1}$, and the thermal conductivity is $K_{e}=K_{0} \frac{T_{e}}{T_{l}}$ with $K_{0}=94 \mathrm{Wm}^{-1} \mathrm{~K}^{-1}$. The system is irradiated by a 200 fs laser pulse which is represented by a source term given by Eq. (16) and added to the TTM equation for electron temperature. An optical penetration depth of $8.9 \mathrm{~nm}$ at a laser wavelength of $400 \mathrm{~nm}$ [163] is assumed in the simulations. The reflectivity of the surface is not defined in the model since the absorbed laser fluence rather than the incident fluence is used in the discussion of the simulation results.

\subsubsection{Computational setup in large-scale simulation}

Large-scale TTM-MD simulation of laser interactions with (001) Cr target is performed for a computational cell with dimensions of $100 \mathrm{~nm} \times 100 \mathrm{~nm} \times 150 \mathrm{~nm}$, consisting of $\sim 126$ million atoms. Periodic boundary conditions are used in the directions parallel to the (100) free surface of the bcc crystal. At the bottom of each MD part, a dynamic pressure-transmitting boundary condition is imposed in order to avoid the reflection of the pressure wave propagating from the irradiated surface of the MD part into the bulk during the laser-induced stresses wave $[110,130]$. Before applying the laser irradiation, the system is equilibrated at $300 \mathrm{~K}$. 
In the continuum part of the model, the heat transfer in the deeper part of the target, which is described by the TTM equations for lattice and electron temperatures, is solved down to the depth of $1 \mu \mathrm{m}$. This choice of the size of the continuum (TTM) region is to ensure the absence of any significant increase in the electron or lattice temperatures at the bottom of the computational domain by the end of the simulation. The parameters that are used in the TTM equations are similar to the ones in the small-scale simulations described above. The system is irradiated by a $50 \mathrm{fs}$ laser pulse at an absorbed laser fluence of $1100 \mathrm{~J} / \mathrm{m}^{2}$, which is above the threshold for the transition from the melting to the photomechanical spallation regimes. An optical penetration depth of $14.95 \mathrm{~nm}$ at a laser wavelength of $800 \mathrm{~nm}$ [163] is assumed in the simulation.

\subsection{Irradiation regimes in femtosecond laser pulse interactions with $\mathrm{Cr}$ target}

In this section, the threshold values of the laser fluence that correspond to the transitions between deferent regimes of material response to the laser irradiation are first identified from the analysis of the fluence dependence of the total ablation yield and the yield of individual (vaporphase) atoms. The mechanisms of the fast laser melting and resolidification, the photomechanical spallation/sputtering of layer(s)/droplets of liquid material, and the phase explosion of an overheated surface region of the target are then discussed based on the results of three representative simulations performed in the corresponding irradiation regimes.

\subsubsection{Ablation yield and composition of the ablation plume}

The simulations of irradiation of a bulk Cr target with a 200 fs laser pulse are performed for absorbed laser fluences ranging from 430 to $383 \mathrm{~J} / \mathrm{m}^{2}$. This range of fluences covers three regimes of material response to laser irradiation: surface melting and resolidification, photomechanical spallation, and phase explosion of an overheated surface region of the target. These regimes can be identified from the fluence dependence of the total amount of material removed from the target (total ablation yield) and the number of ejected individual (vapor-phase) atoms, shown in Fig. 2.1.

At low laser fluences, between the thresholds for surface melting, $500 \mathrm{~J} / \mathrm{m}^{2}$ [129] and spallation, $\sim 950 \mathrm{~J} / \mathrm{m}^{2}$, the laser induced processes are limited to the melting and resolidification of a part of the surface region. The depth of the transiently melted surface region is increasing 
with increasing laser fluence and reaches $23 \mathrm{~nm}$ at the highest fluence of $850 \mathrm{~J} / \mathrm{m} 2$ simulated in this regime. Only 3 atoms, however, are observed to evaporate from the surface of the target at this fluence.

The increase of the laser fluence above $950 \mathrm{~J} / \mathrm{m}^{2}$ results in a separation of a layer of melted material from the bulk of the target, which is reflected in a step increase in the total ablation yield in Fig. 2.1(a). As discussed in Section 2.3.2, the ejection of the liquid layer is driven by the relaxation of the laser-induced stresses generated in the surface region of the target under conditions of stress confinement. Therefore, by analogy with the term "spallation," commonly used to describe the dynamic fracture that results from the reflection of a shock wave from a back surface of a sample $[164,165]$, the material ejection due to the laser-induced stresses is called here front-surface laser spallation [152].
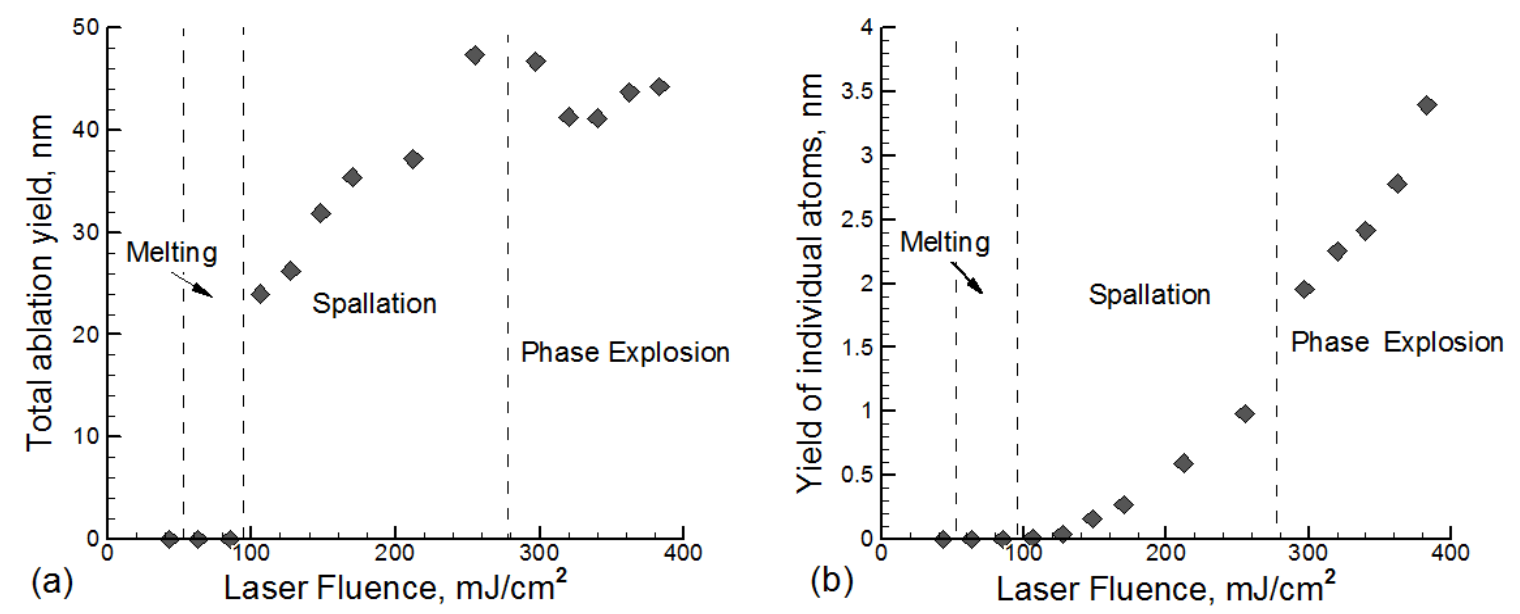

Fig. 2.1. Total ablation yield (a) and number of individual atoms in the ablation plume (b) as functions of absorbed laser fluence predicted in simulations for bulk Cr targets irradiated with $200 \mathrm{fs}$ laser pulses. The values of the total yield and the yield of individual atoms are expressed in units of depth in the initial target (number of ejected atoms is equivalent to the number of atoms in a layer of this depth in the initial target). The dashed lines mark the approximate values of the threshold fluences for surface melting, spallation and phase explosion. 
An increase of the laser fluence above the spallation threshold results in the separation and ejection of multiple layers/droplets from the target. At the higher fluences, but still in the spallation regime, the surface layer is strongly overheated and readily disintegrates at moderate tensile stresses. The number of vapor phase atoms evaporating from the ejected liquid layers and droplets also increases with fluence but remains below $2 \%$ of the total yield, Fig. 2.1(b). The temperature of the surface region of the target in the spallation regime stays below the temperature required for the onset of the explosive boiling. The threshold temperature for the onset of the phase explosion can be determined in constant-pressure MD simulations of a slow heating of a metastable liquid. This approach has been used earlier to determine the threshold temperature for the onset of the explosive boiling in a system of Ar atoms [166], a molecular system represented by the breathing sphere model [167], and a Ni material simulated with EAM potential [120], The threshold temperature for the phase explosion can be expected to be $\sim 10 \%$ below the critical temperature of the material $[23,144,145,166]$. In this work, we perform a similar set of simulations for a cubic system of $6500 \mathrm{Cr}$ atoms. Periodic boundary conditions are used in all directions to simulate a small region inside a bulk material. The change in volume of the system as a function of temperature, obtained in these simulations, is shown in Fig. 2.2. The threshold temperature for the onset of the phase explosion can be identified from a sharp increase of the volume of the system that corresponds to the explosive phase separation into liquid and vapor. This threshold temperature is found to be about $6000 \mathrm{~K}$ at zero pressure for the EAM $\mathrm{Cr}$ material.

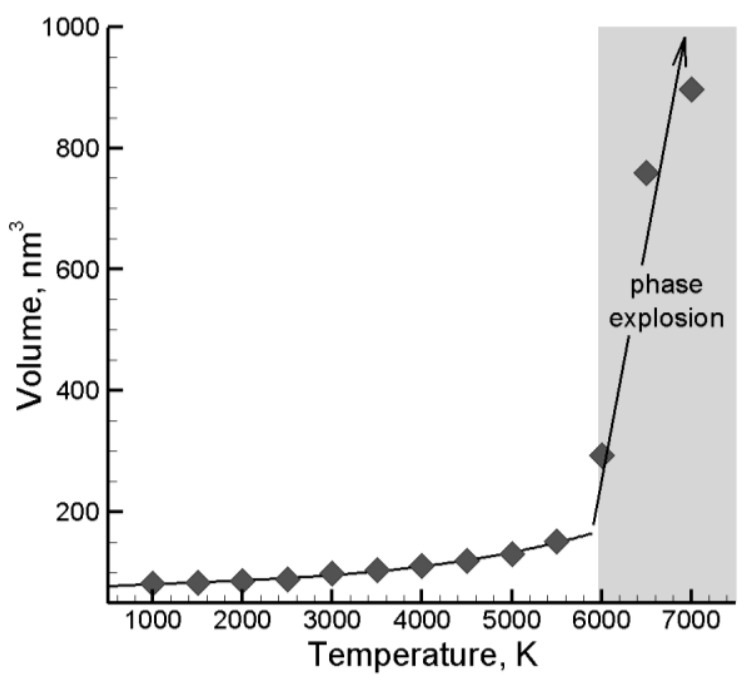

Fig. 2.2. Temperature dependence of the volume of a computational cell in MD simulations of a slow constant-pressure heating of a cubic system of liquid $\mathrm{Cr}$. The results of the simulations are used to determine the threshold temperature for the onset of phase explosion in the EAM $\mathrm{Cr}$ material. 
At laser fluences that bring the surface region of the irradiated target to temperatures that reach and exceed the threshold temperature for the phase explosion, the dominant mechanism responsible for the material ejection changes from the photomechanical spallation driven by the relaxation of the laser-induced stresses to the phase explosion driven by the rapid decomposition of the overheated material into a mixture of vapor and liquid droplets. The transition to the phase explosion regime does not result in an increase in the total amount of the ejected material, Fig. 2.1(a). Quite the reverse, the total yield decreases somewhat and saturates at fluences above the threshold for the phase explosion. The decrease of the total yield can be explained by two factors: (1) a higher energy cost of the decomposition of the surface region of the target into a mixture of vapor and small liquid droplets as compared to the ejection of larger droplets in the spallation regime, and (2) redeposition of some of the droplets ejected at end of the ablation process back to the target due to the vapor pressure from the upper part of the plume. Indeed, deceleration and redeposition of some of the large droplets is observed in simulations performed at laser fluences of $3400 \mathrm{~J} / \mathrm{m}^{2}$ and $3620 \mathrm{~J} / \mathrm{m}^{2}$.

The transition to the phase explosion regime is also signified by an increase in the number of vapor phase atoms in the ablation plume, Fig. 2.1(b). Given that the total yield saturates and even decreases upon the transition to the phase explosion regime, the fraction of the vapor phase in the ablation plume increases from $\sim 2 \%$ of the total yield right below the threshold for the phase explosion to $4-7 \%$ above the threshold. This increase is less dramatic as compared to the one observed in the simulations performed for Ni targets [120], where the jump in the fraction of the vapor-phase atoms was from $2 \%$ to more than $10 \%$ of the total yield. The difference may be related to the material-dependent characteristics of the phase explosion process in $\mathrm{Cr}$ and $\mathrm{Ni}$.

The increase in the fraction of the vapor-phase atoms upon the transition from the spallation to the phase explosion regime can be related to the results of plume imaging experiments [168], where the maximum ejection of nanoparticles in laser ablation of Ni targets is observed at low fluences (possible spallation), whereas the degree of the plume atomization increases at higher fluences (possible phase explosion regime). The transition from spallation to phase explosion has also been related [120] to the results of pump-probe experiments [151, 169], where the observation of optical interference patterns (Newton rings) can be explained by the 
spallation of a thin liquid layer from the irradiated target $[152,228,170]$ and the disappearance of the interference fringes in the central part of the laser spot [151] may be related to the transition to the phase explosion regime.

\subsubsection{Mechanisms of melting, spallation, and phase explosion}

The mechanisms of melting, spallation, and phase explosion are discussed in this Section based on the results of three representative simulations performed in each of these regimes. The process of melting and recrystallization is illustrated in Figs. 2.3 and 2.4 by the results of the simulation performed at an absorbed laser fluence of $850 \mathrm{~J} / \mathrm{m}^{2}$, about $70 \%$ above the threshold for surface melting [129].

The temporal and spatial evolution of the lattice temperature and pressure in the surface region of the target is shown in the form of contour plots in Fig. 2.3. The temperature plot in Fig. 2.3(a) shows that the electronic excitation by the $200 \mathrm{fs}$ laser pulse and the energy transfer to the lattice due to the electron-phonon coupling result in a fast lattice heating during the first several picoseconds after the laser irradiation. The maximum temperature reached by $5.5 \mathrm{ps}$ at a depth of 10 to $20 \mathrm{~nm}$ below the initial surface exceeds $3200 \mathrm{~K}$, which is $37 \%$ above the equilibrium melting temperature of the EAM Cr material, $T_{m}=2332 \mathrm{~K}$ (this value is obtained by liquidcrystal coexistence simulations performed under constant-pressure conditions and is slightly lower than the value of $2381 \mathrm{~K}$ reported earlier in Ref. [129]. At this overheating, fast homogeneous melting takes place and proceeds in a form of the fast collapse of the crystal lattice without the formation of well-defined liquid nuclei [118, 143, 161, 162]. The fast homogeneous melting of $\sim 20 \mathrm{~nm}$ surface region is followed by an additional slow propagation of the melting front, formed by the end of the homogeneous melting process, deeper into the target (heterogeneous melting). The transfer of the thermal energy to the latent heat of melting, cooling due to the expansion of the surface region, and the fast electronic heat conduction to the bulk of the target result in a rapid cooling of the surface region. The temperature near the liquid-crystal interface drops below the equilibrium melting temperature by the time of $50 \mathrm{ps}$ and the melting turns into epitaxial recrystallization of the melted region. 

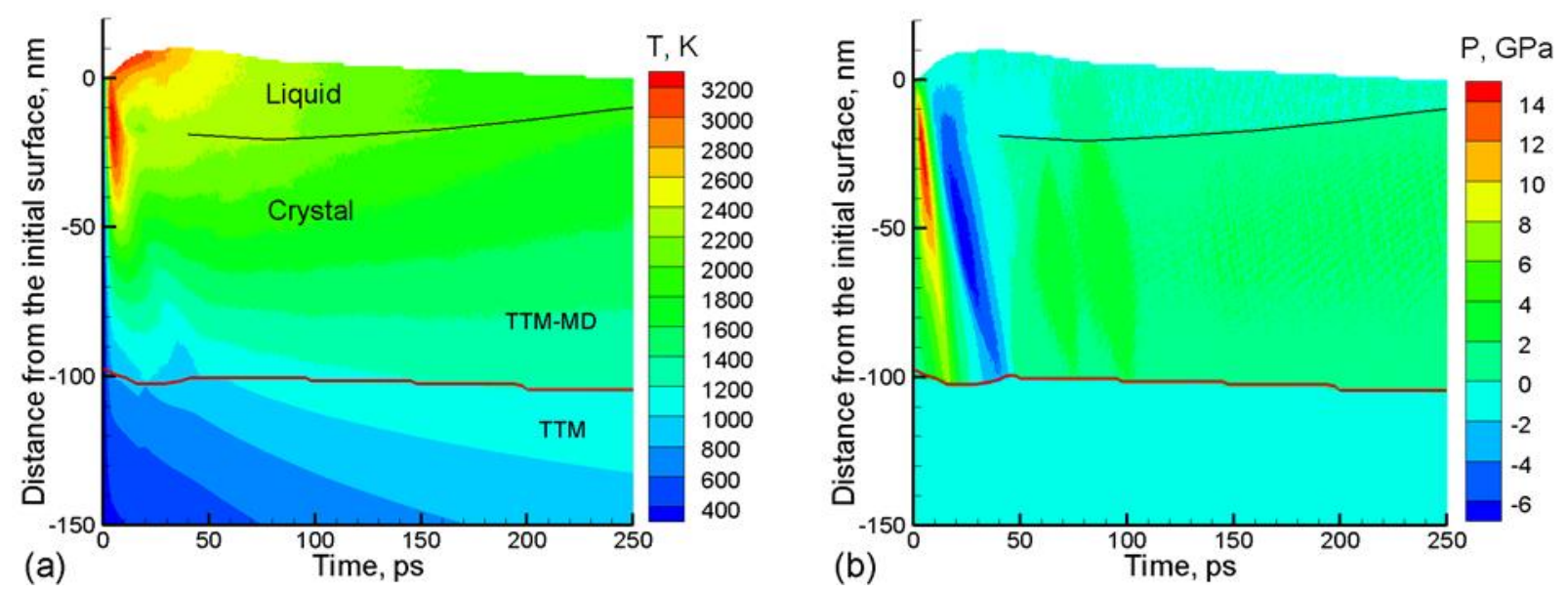

Fig. 2.3. Contour plots of the spatial and temporal evolution of (a) lattice temperature and (b) pressure in a TTM-MD simulation of a bulk Cr target irradiated with a $200 \mathrm{fs}$ laser pulse at an absorbed fluence of $850 \mathrm{~J} / \mathrm{m}^{2}$. The laser pulse is directed along the $\mathrm{Y}$ axes, from the top of the contour plots as shown by the red arrow. The black solid lines separate the melted and crystalline regions of the target. The red solid lines separate the continuum (TTM) and atomistic (TTM-MD) parts of the computational system.

The velocity of the solidification front increases with increasing undercooling below the melting temperature and reaches the maximum value of about $80 \mathrm{~m} / \mathrm{s}$ by the time when the melting front reaches the surface of the target and the temperature at the liquid-crystal interface drops down to about $0.8 T_{m}$. The values of the solidification velocity observed in the simulations are comparable to the ones estimated from pump-probe measurements performed for Ag films [142].

A visual picture of the melting and resolidification of the surface region of the target is provided by snapshots of atomic configurations shown in Fig. 2.4. The atoms in the snapshots are colored according to their potential energy and the red regions correspond to the melted part of the target, where the average atomic energy is elevated by the latent heat of melting. The melting process during the first $50 \mathrm{ps}$ is followed by resolidification, which proceeds by the propagation of a plane solidification front towards the surface. The solidification front reaches the surface by the time of $330 \mathrm{ps}$, leaving behind a region of the target supersaturated with vacancies. 


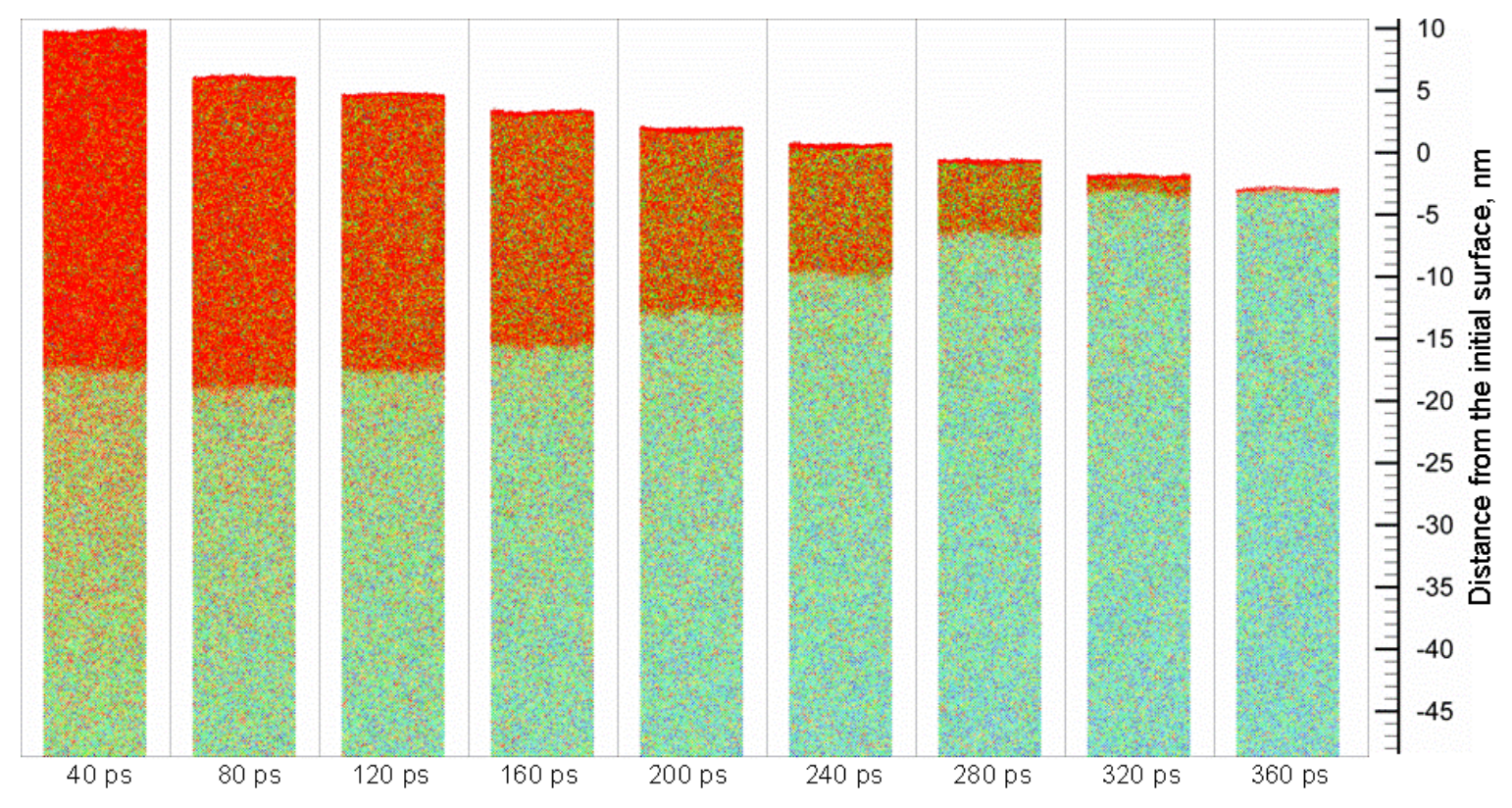

Fig. 2.4. Snapshots of atomic configurations obtained in a TTM-MD simulation of a bulk Cr target irradiated with a $200 \mathrm{fs}$ laser pulse at an absorbed fluence of $850 \mathrm{~J} / \mathrm{m}^{2}$. The snapshots are taken along the [100] view direction. The atoms are colored according to their potential energy; the red region corresponds to the elevated average atomic energy in the melted region.

The continuing shift of the system in the downward direction long after the laser pulse (e.g., the solidified surface is below the initial surface in the final snapshots shown in Fig. 2.4) is an artifact of the non-reflecting boundary condition that is not fully optimized for the potential used in the simulations. This slow downward drift of the computational cell, however, does not affect any of the results discussed in this section.

The rapid increase in the lattice temperature during the first picoseconds after the laser pulse takes place under conditions of the inertial stress confinement $[113,149,152,171]$ and results in the buildup of compressive stresses in the surface region of the irradiated target, Fig. 2.3(b). The compressive stresses increase during the time of the lattice heating and reach a maximum value of $\sim 14 \mathrm{GPa}$ by the time of $5 \mathrm{ps}$ after the laser excitation. The relaxation of the compressive stresses in the presence of the free surface of the target results in the generation of a bimodal stress wave that propagates deeper into the bulk of the target. The wave consists of a compressive component followed by a tensile one, Fig. 2.3(b). The pressure-transmitting heatconductive boundary condition, applied at the depth of $100 \mathrm{~nm}$, ensures that both the 
compressive and tensile components of the pressure wave propagate without any significant reflection from the boundary separating the TTM-MD and TTM parts of the model (Fig. 2.3b), whereas the temperature field exhibits a seamless transition at the boundary (Fig. 2.3a).

The strength of the compressive and tensile stresses generated by the laser pulses increases with increasing laser fluence and, in a simulation performed at a fluence of $1060 \mathrm{~J} / \mathrm{m}^{2}$, the tensile stresses exceed the dynamic strength of the melted material and cause spallation or separation of a melted layer from the target, Fig. 2.5. The microscopic mechanisms of spallation, which proceeds through the nucleation, growth, coalescence, and percolation of multiple voids in a surface region of the target, are discussed in detail in Ref. [152]. The general characteristics of the spallation process, including the evolution of the void size distributions, appear to be common for different materials, including molecular systems [113, 152, 172] and metals [120, $151,173]$. Some of the features of the spallation process are apparent in the contour plots shown in Fig. 2.5. In particular, the nucleation and growth of voids is observed in the melted region of the target, at a depth that is much closer to the surface as compared with the depth where the maximum tensile stresses are generated. This is explained by the reduced ability of the overheated liquid to support transient tensile stresses generated by the laser-induced wave [152]. The spallation separates a hot surface layer from the bulk of the target and substantially accelerates the resolidification of the remaining part of the target. Indeed, the solidification front reaches the surface by 200 ps in Fig. 2.5 and by 330 ps in Fig. 2.3. A similar effect of spallation on the duration of the solidification process is also reported in Ref. [120] for Ni targets. 

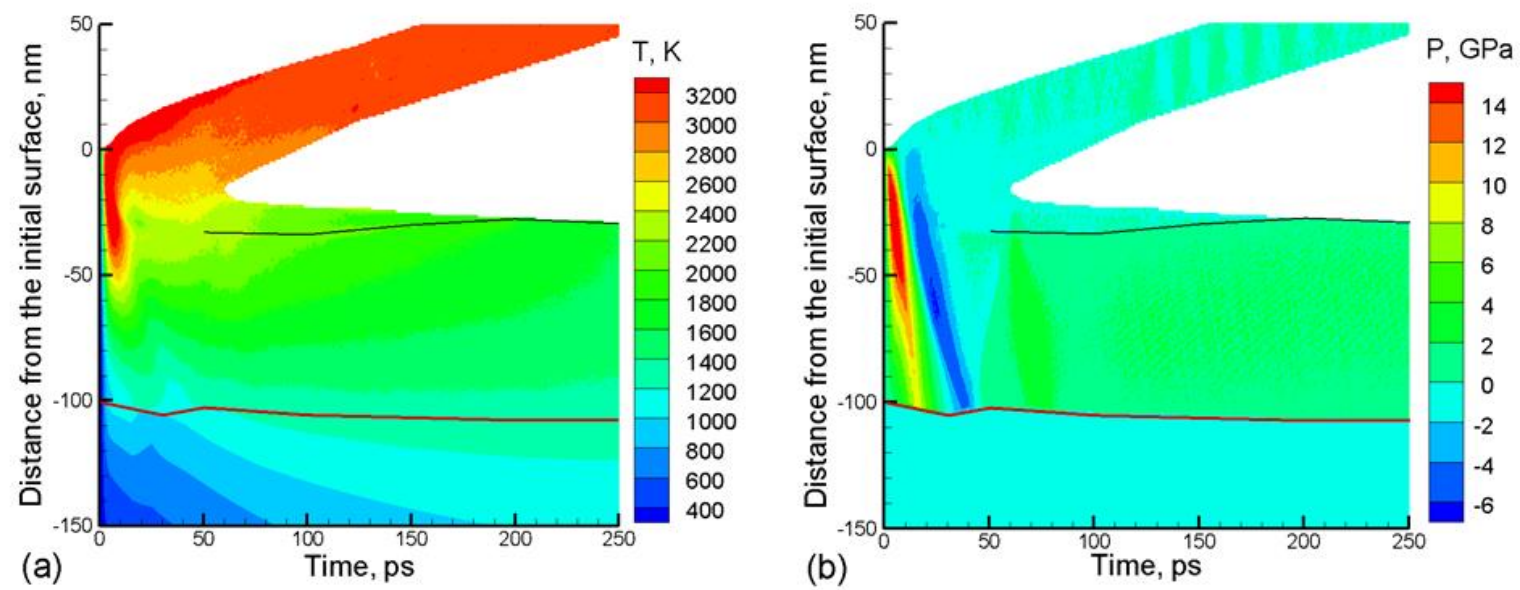

Fig. 2.5. Contour plots of the spatial and temporal evolution of (a) lattice temperature and (b) pressure in a TTM-MD simulation of a bulk Cr target irradiated with a $200 \mathrm{fs}$ laser pulse at an absorbed fluence of $1060 \mathrm{~J} / \mathrm{m}^{2}$, just above the spallation threshold. The laser pulse is directed along the Y axes, from the top of the contour plots. The black solid lines separate the melted and crystalline regions of the target. The red solid lines separate the continuum (TTM) and atomistic (TTM-MD) parts of the computational system.

Further increase of the laser fluence above the spallation threshold results in the separation and ejection of multiple layers/droplets from the target and, above $\sim 2750 \mathrm{~J} / \mathrm{m}^{2}$, leads to the transition from the regime of photomechanical spallation to the phase explosion characterized by a rapid decomposition of the overheated material into a mixture of vapor and liquid droplets. This transition occurs when the surface region of the irradiated target reaches and exceeds the threshold temperature for the onset of the phase explosion identified to be around $6000 \mathrm{~K}$ in Section 2.2.1, see Fig. 2.2. A surface region where temperature exceeds the threshold temperature for the phase explosion becomes unstable and disintegrates in an explosive manner. An example illustrating this ablation mechanism is shown in Fig. 2.6, where the temperature and pressure contour plots are shown for a simulation performed at an absorbed fluence of $2980 \mathrm{~J} / \mathrm{m}^{2}$.

The transition to the regime of phase explosion not only alters the composition of the ejected plume (increases the fraction of the vapor phase, Fig. 2.1), but also changes the dynamics of the ablation plume expansion and its interaction with the target. The vapor pressure released in the explosive decomposition of the top part of the target slows down the expansion of lower part of the ejected plume and prolongs its interaction with the new surface of the target. This interaction slows down the cooling of the surface and significantly increases the time scales of the melting and resolidification processes, compare Figs. 2.5 and 2.6. The transition from the 
spallation to the phase explosion regime also affects the characteristics of the pressure wave generated by the laser irradiation, Fig. 2.6(b). While the compressive component of the pressure wave continues to increase linearly with increasing laser fluence, the tensile component starts to decrease in the spallation and phase explosion regimes. This decrease can be attributed to the reduced ability of the strongly overheated part of the target to support the transient tensile stresses, as well as to the compressive ablation recoil pressure that partially cancels the tensile component of the wave.

To illustrate the process of the decomposition of the overheated material in the regime of phase explosion, snapshots of the ablation plum, taken at the same height above the initial surface, are shown in Fig. 2.7 for different times marked in the contour plot in Fig. 2.6(a). The first snapshot, shown for 20 ps in Fig. 2.7(a), provides an atomic-level picture of the homogeneous expansion of the strongly overheated top layer of the target. The homogeneous expansion is followed by the appearance and growth of density fluctuations and formation of a foamy transient structure of interconnected liquid regions surrounded by vapor-phase atoms, Fig. 2.7(b). The foamy transient structure subsequently decomposes into separate elongated liquid droplets, Fig. 2.7(c), which gradually develop into spherical droplets in a process driven by the minimization of surface energy of the droplets. 

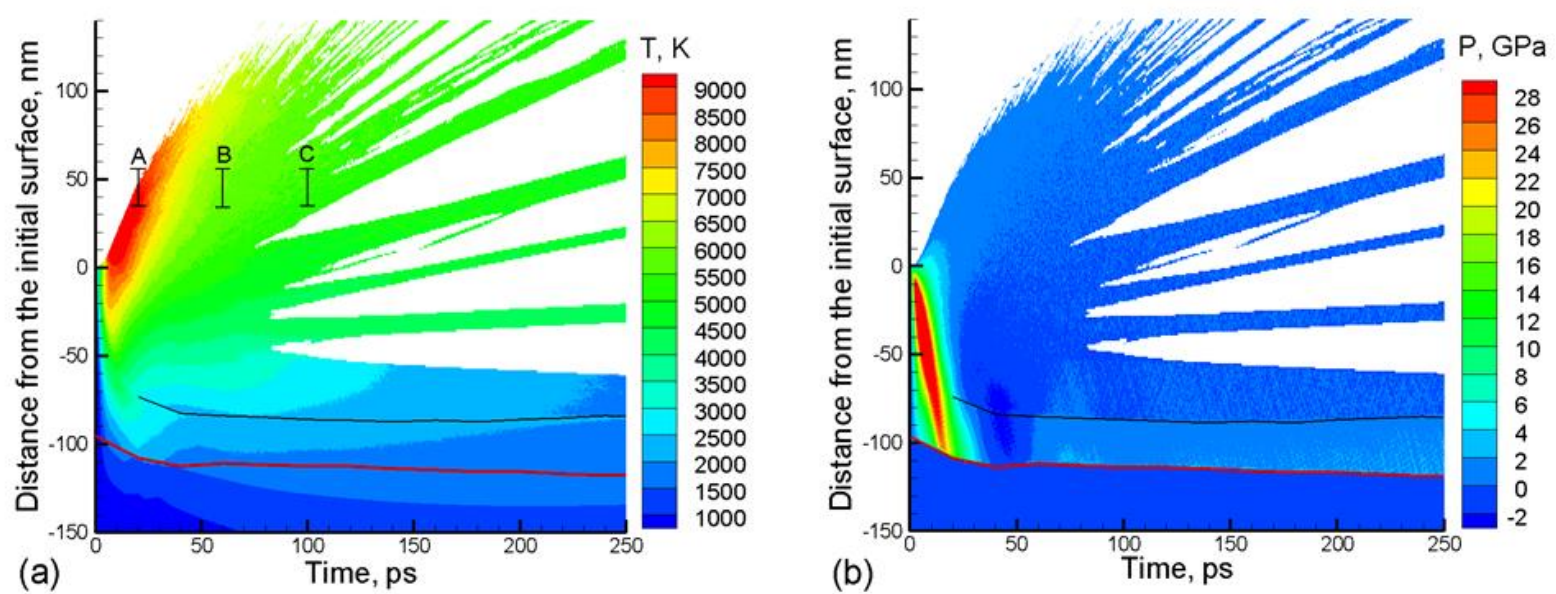

Fig. 2.6. Contour plots of the spatial and temporal evolution of (a) lattice temperature and (b) pressure in a TTM-MD simulation of a bulk Cr target irradiated with a $200 \mathrm{fs}$ laser pulse at an absorbed fluence of $2980 \mathrm{~J} / \mathrm{m}^{2}$, just above the threshold for the phase explosion. The laser pulse is directed along the $\mathrm{Y}$ axes, from the top of the contour plots. The black solid lines separate the melted and crystalline regions of the target. The red solid lines separate the continuum (TTM) and atomistic (TTM-MD) parts of the computational system. Marks A, B, and C show the time and locations in the ablation plume for which snapshots of atomic configurations are shown in Fig. 2.7.

The dynamics of the material ejection and the composition of the ablation plume predicted in the simulations performed in the phase explosion regime are affected by the conditions of the stress confinement created by the fast laser energy deposition. The phase explosion occurring under conditions of stress confinement takes place simultaneously with the relaxation of the laser-induced stresses, resulting in a more vigorous material ejection, higher ablation yields, and smaller fraction of the vapor phase as compared to the "pure" phase explosion, characteristic for longer pulses. A similar effect of the stress confinement has been observed earlier in MD simulations of laser ablation Ni targets [120] and molecular systems $[113,114]$, where a higher ablation yields, larger droplets, smaller fractions of the vapor phase, and higher ejection velocities are observed in simulations performed for irradiation conditions that correspond to the stress confinement. A relatively weak phase explosion, on the other hand, has been reported in MD simulations of nanosecond laser ablation of a Lennard-Jones system [174], where vapor phase molecules are found to account for the largest fraction of the ablation yield. 


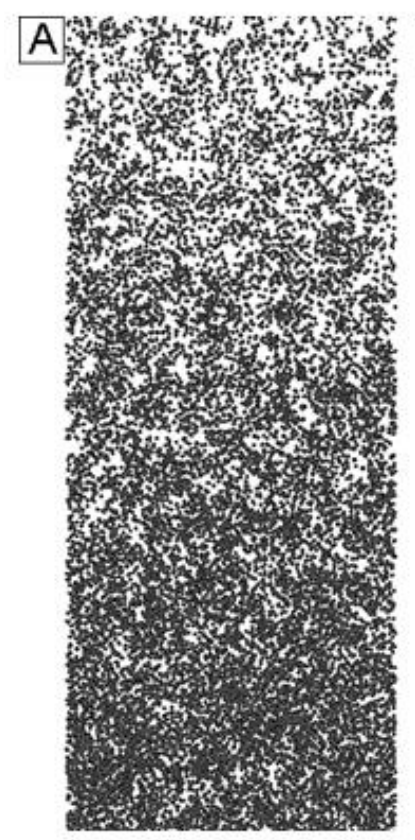

$20 \mathrm{ps}$

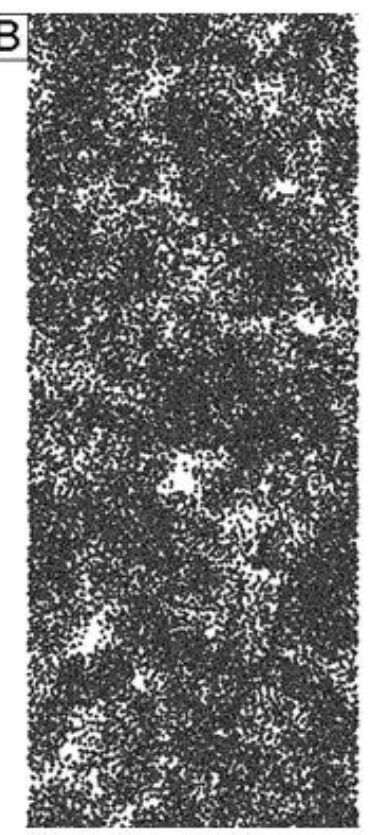

$60 \mathrm{ps}$
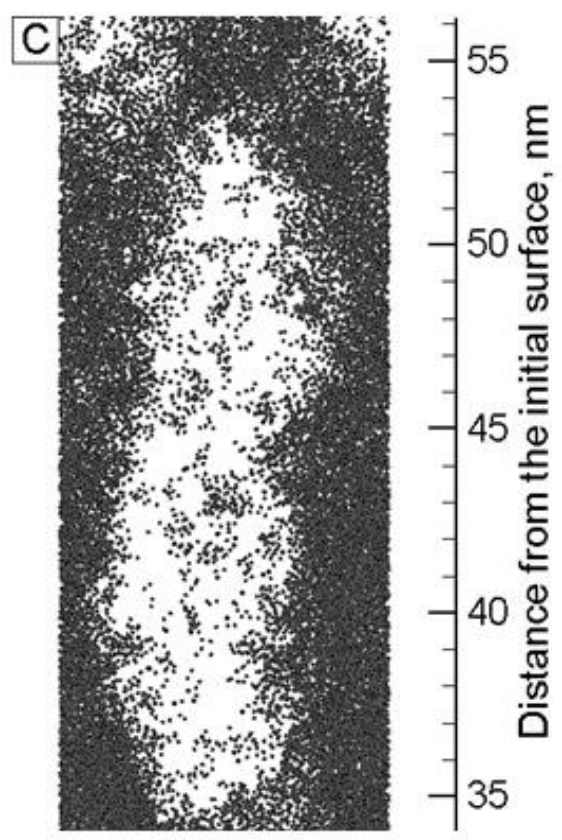

$100 \mathrm{ps}$

Fig. 2.7. Snapshots of atomic configurations obtained in a TTM-MD simulation of a bulk Cr target irradiated with a $200 \mathrm{fs}$ laser pulse at an absorbed fluence of $2980 \mathrm{~J} / \mathrm{m}^{2}$, close to the threshold for the phase explosion. Snapshots are taken at times and locations marked in the temperature contour plot in Fig. 2.6 (a). The snapshots are taken along the [010] view direction.

\subsubsection{A microscopic view of the mechanisms of laser-induced photomechanical spallation from a large-scale atomistic simulation}

Although the results of the simulations of laser-induced photomechanical spallation discussed in Section 2.3.2 have revealed the general physical picture of the spallation process, the small lateral size of the computational system used in the simulations, $8 \times 8 \mathrm{~nm}^{2}$, does not permit a realistic representation of the nucleation, growth, and percolation of voids responsible for the spallation phenomenon. Therefore, an additional large-scale MD simulation of a $50 \mathrm{fs}$ laser pulse interactions with (001) Cr target is performed for a system with lateral sizes increased to $100 \times 100 \mathrm{~nm}^{2}$. The simulation is carried out at an absorbed laser fluence of $1100 \mathrm{~J} / \mathrm{m}^{2}$, which is slightly above the threshold for the transition from melting to the photomechanical spallation regimes. 
The fundamental picture of the mechanisms responsible for the laser-induced photomechanical spallation has not been changed in the large-scale simulation of the $\mathrm{Cr}$ target irradiated by a short laser pulse as can be seen from the comparison of the contour plots of the spatial and temporal evolution of the lattice temperature, pressure and density in the surface regions of the irradiated targets shown in Figs. 2.5 and 2.8 for the small- and large-scale simulations, respectively. Succinctly, in the large-scale simulation, the fast deposition of the laser energy in the surface region of the irradiated target leads to a rapid increase of the lattice temperature from $300 \mathrm{~K}$ to $3700 \mathrm{~K}$ during the first $5 \mathrm{ps}$ after the laser pulse and causes a prompt melting of a $36 \mathrm{~nm}$ surface region of the target. The rapid lattice heating takes place under conditions of the inertial stress confinement and results in the generation of strong compressive and tensile stresses as can be seen from the pressure contour plot in Fig. 2.8(b). In this simulation, the tensile stress exceeds the dynamic strength of the melted material and leads to nucleation and growth of voids inside the melted region of the target. The appearance of voids is reflected in the density contour plot shown in Fig. 2.8(c), where the reduction of density is first observed at about $30 \mathrm{ps}$ after the laser pulse, the low-density region expands with time, and a complete separation of a melted layer from the target is observed at about $800 \mathrm{ps}$. 

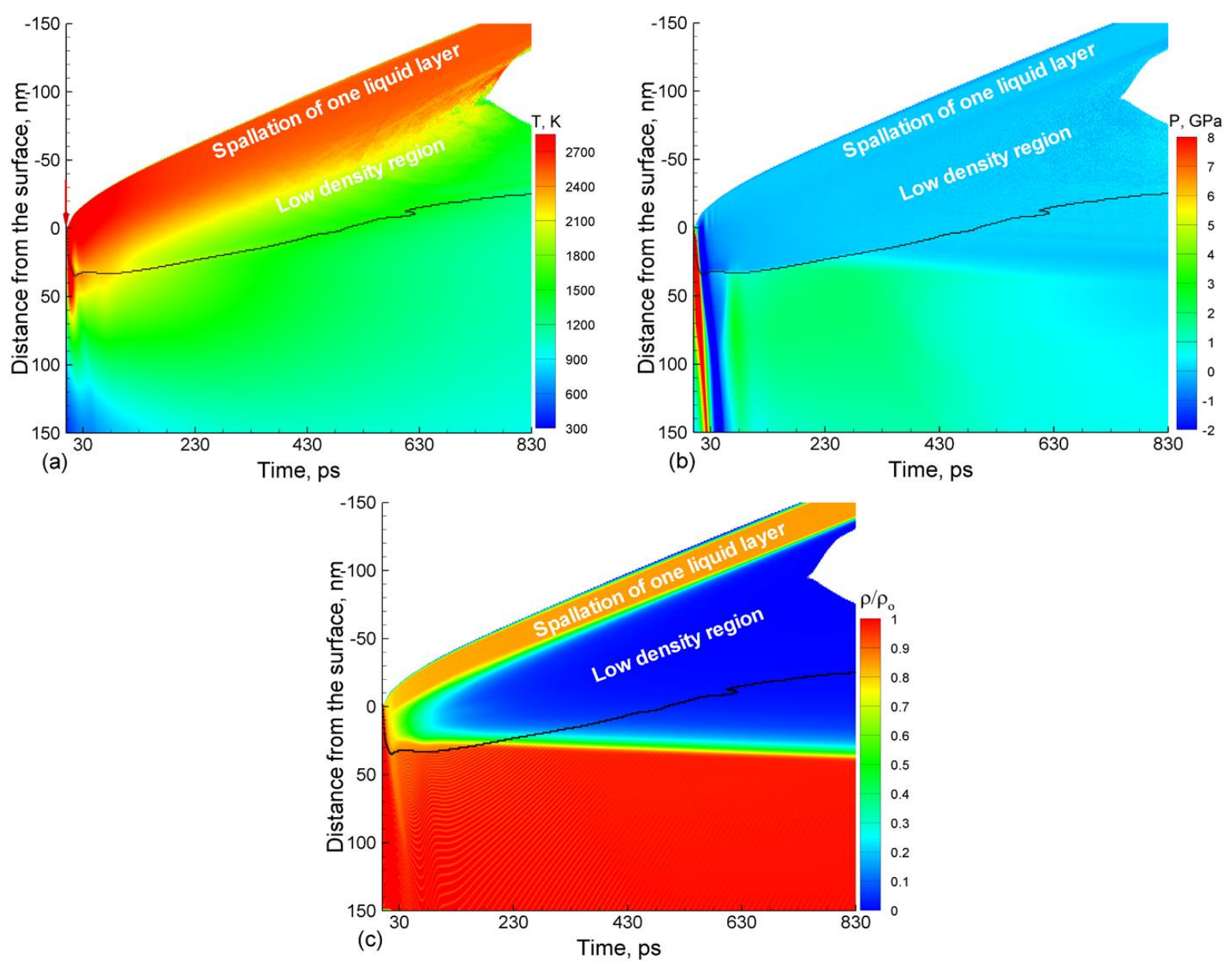

Fig. 2.8. Contour plots of the spatial and temporal evolution of (a) the lattice temperature, (b) pressure, and (c) density in a large scale TTM-MD simulation of a bulk (001) $\mathrm{Cr}$ target irradiated with a 50 fs laser pulse at an absorbed laser fluence of $1100 \mathrm{~J} / \mathrm{m}^{2}$, slightly above the spallation threshold. The laser pulse is directed along the Y-axis as shown by the red arrow in (a). The black line separates the melted region from the crystalline part of the $\mathrm{Cr}$ target.

In order to capture the evolution of multiple voids in the surface region of the target, snapshots of atomic configurations obtained in the large-scale TTM-MD simulation of Cr target are taken at different simulation times and shown in Fig. 2.9. Each atomic configuration is quenched to $0 \mathrm{~K}$ for 2 ps using the velocity damping technique. In this technique, the velocity of each individual atom is set to zero when the kinetic energy of the atom reaches its maximum value. This technique helps to reduce the thermal noise in the atomic positions and energies without introducing any changes in the structure of the atomic configuration. The atoms are then colored according to their potential energies where the dark blue region corresponds to the bcc 
$\mathrm{Cr}$ structure, green region represents the liquid phase, and the atoms at the top surface of the target and at the internal surfaces appearing in the spallation process are colored red. The light blue stripes that appear in the bulk $\mathrm{Cr}$ target by the time of $\sim 50 \mathrm{ps}$ and disappear at later time represent islands of unstable stacking faults generated in response to the uniaxial expansion caused by the dynamic relaxation of the laser-induced stresses. A detailed discussion of the transient generation of the unstable stacking faults in (001) $\mathrm{Cr}$ targets is provided in Section 3.5.1.

The snapshots shown in Fig. 2.9 illustrate nucleation of a large number of sub-surface voids followed by their growth, coalescence, and eventual percolation leading to the separation and ejection of a liquid layer. In contrast with the small-scale simulation, where the voids quickly "outgrow" the lateral size of the computational cell and result in a prompt separation of a liquid layer shortly after the void nucleation (Fig. 2.5), the evolution of voids in the larger computational system takes longer time and proceeds through the formation of a transient foamy structure of interconnected liquid regions, or bridges, connecting the upper 27-nm-thick liquid layer with the rest of the target. Gradually with the advancement of the simulation time, the liquid bridges are separated from the liquid layer that continues to move away and eventually complete separates from the target by the time 800 ps.

The detailed analysis of the snapshots in Fig. 2.9 demonstrates the release of only a small number of gas phase atoms inside the growing voids which indicates that the driving force for the nucleation and growth of the voids is the relaxation of the laser-induced thermoelastic stresses rather than the vapor pressure in the boiling process. Several molecular dynamics studies focused on investigation the mechanisms of laser-induced photomechanical spallation suggest that this phenomenon can take place in a wide range of materials and may have similar features in systems as different as molecular solids and metals [24, 60, 152]. 


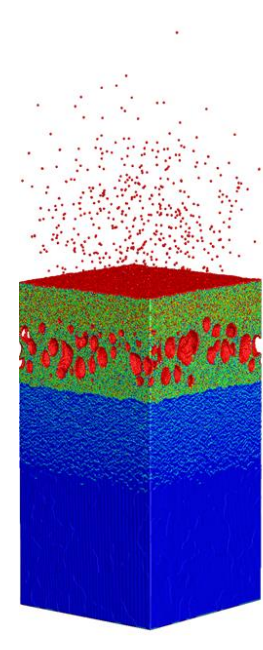

50 ps

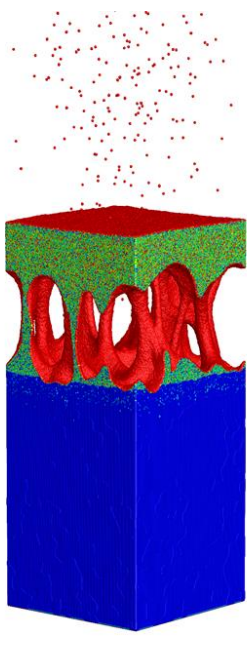

200 ps
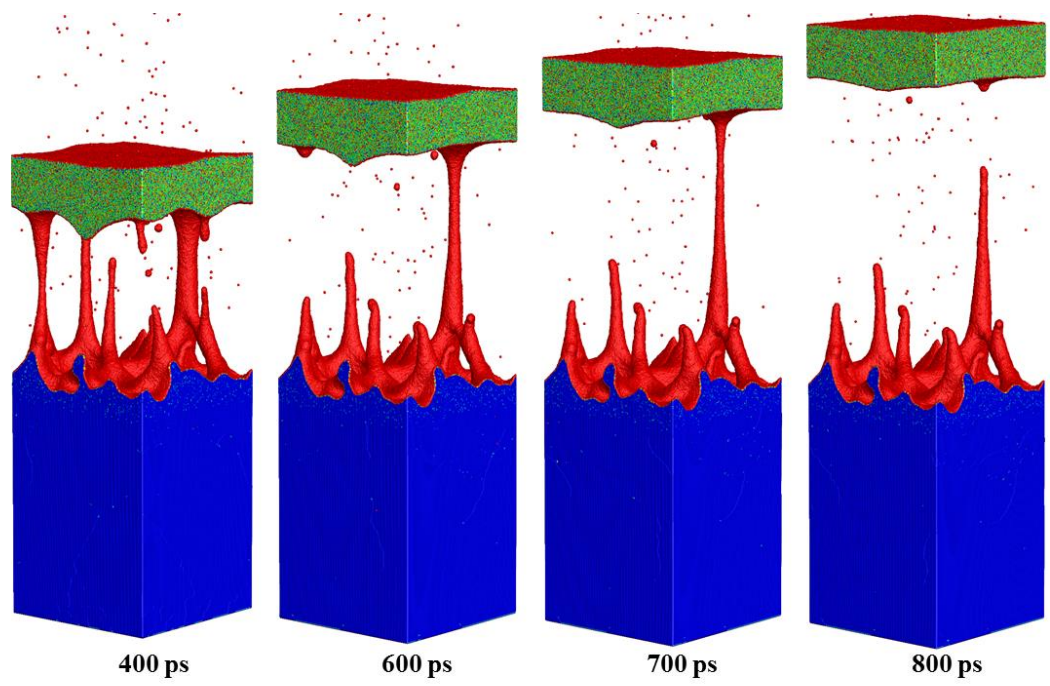

Fig. 2.9. Snapshots of atomic configurations obtained in a TTM-MD simulation of laser-induced photomechanical spallation of a bulk (001) Cr target irradiated with a 50 fs laser pulse at an absorbed laser fluence of $1100 \mathrm{~J} / \mathrm{m}^{2}$. The laser irradiation is directed from the top of the images. The atoms are colored according to their potential energies: the dark blue region represents the bcc $\mathrm{Cr}$ structure, green region represents the liquid phase, and the atoms at the top surface of the target and at the internal surfaces appearing in the spallation process are colored red. The light blue stripes that appear in the crystalline part of $\mathrm{Cr}$ target by the time of $50 \mathrm{ps}$ and gradually disappear at later time represent the unstable stacking faults transiently appearing in the system. The snapshots present the evolution of the voids up to the time of a complete spallation of a liquid layer from the bulk of the target.

At $40 \mathrm{ps}$, the temperature near the liquid-solid interface drops below the melting temperature and allows for the epitaxial resolidification to take place. The velocity of the solidification process increases with the increasing undercooling at the solidification front and reaches $\sim 77 \mathrm{~m} / \mathrm{s}$ at a time of $800 \mathrm{ps}$, the time when the melted layer is completely separated (spalled) from the bulk of the target. The fast epitaxial growth results in solidification of nine spikes that are formed during the breakup of the liquid bridges in the spallation process. The height of these spikes ranges from 12 to $130 \mathrm{~nm}$, as can be seen in Fig. 2.10. 


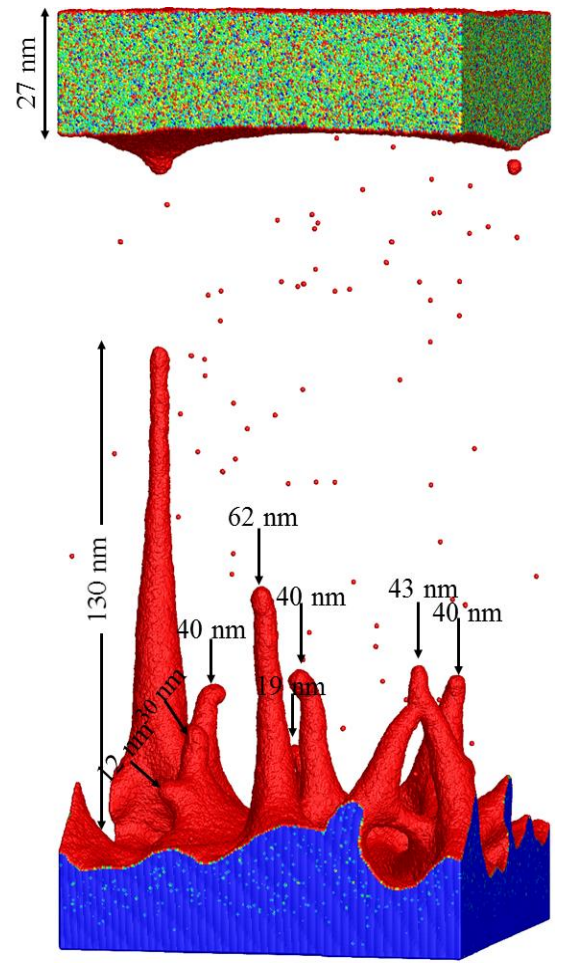

Fig. 2.10. Snapshot of an atomic configuration obtained in a TTM-MD simulation of laser-induced photomechanical spallation of a bulk (001) Cr target irradiated with a $50 \mathrm{fs}$ laser pulse at an absorbed laser fluence of $1100 \mathrm{~J} / \mathrm{m}^{2}$. The snapshot is shown for a time of $800 \mathrm{ps}$, when the top liquid layer is completely separated from the bulk of the target. The snapshot illustrates the formation of nine solidified spikes with the length ranged between 12 and $130 \mathrm{~nm}$. The atoms are colored according to their potential energies with the same coloring scale that is in Fig. 2.9.

The surface morphology of the irradiated target after the complete spallation of the one liquid layer is shown in Fig. 2.11(a). The atoms in this topographic map are colored according to their elevation with respect to the initial surface. The topography of the surface illustrates the formation of several characteristic types of nanostructure such as nanopores, nanovoids, nanoprotrusions, nanocavities, and nanorims. Several factors play a role in the formation of these nanostructures: (1) the evolution of the voids leading to the formation of complex interconnected liquid structures that are gradually detached from the top liquid layer, (2) the high surface tension of $\mathrm{Cr}$ at the melting temperature, which facilitates the formation of the nanostructure, see Fig. 2.12(d), and (3) the rapid advancement of the solidification front result in capturing (freezing) the transient liquid structures in the shapes that appear in Fig. 2.11(a). The surface morphology predicted by the TTM-MD simulation of the $\mathrm{Cr}$ target is consistent with the surface morphologies observed in experiments on short pulse laser processing of metal target. For instance, the results obtained by Vorobyev and Guo for a copper target irradiated by a 65 fs laser pulse at an absorbed laser fluence of $3500 \mathrm{~J} / \mathrm{m}^{2}$ [18, 43].,Fig. 2.11(b), illustrate the formation of surface nanostructures that are very similar to the ones predicted for $\mathrm{Cr}$ target in the atomistic simulation. 


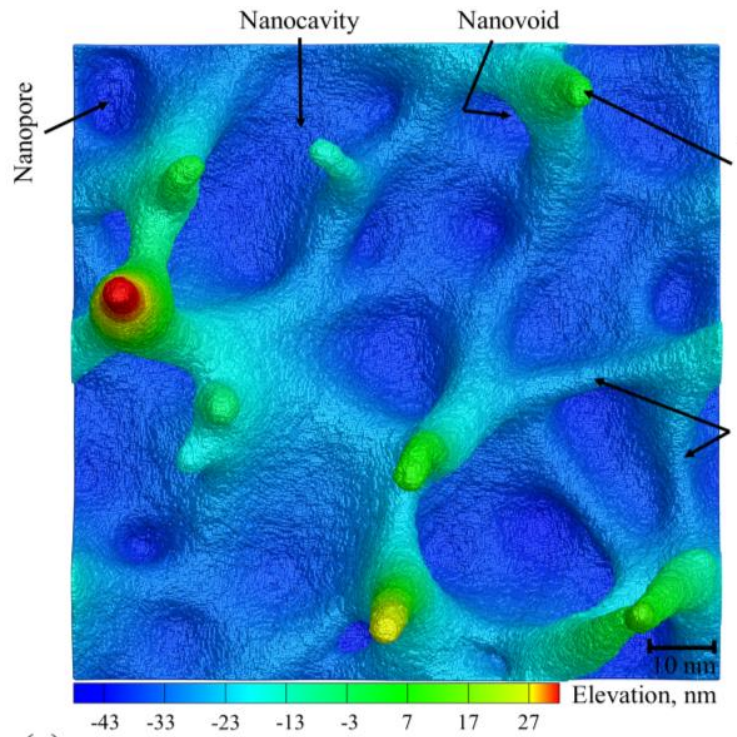

(a)

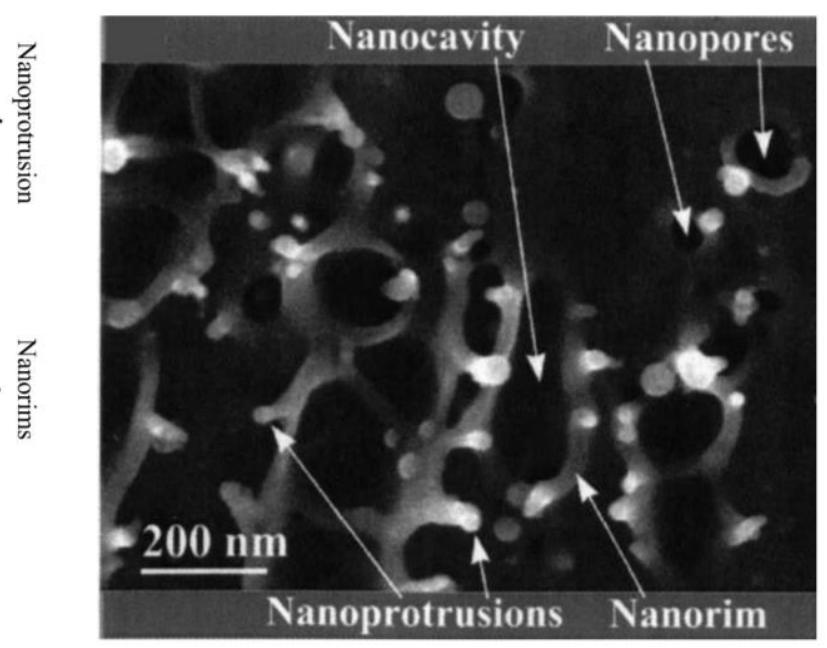

(b)

Fig. 2.11. Surface topography of metal targets obtained in (a) TTM-MD simulation of a bulk (001) $\mathrm{Cr}$ target irradiated with a $50 \mathrm{fs}$ laser pulse at an absorbed laser fluence of $1100 \mathrm{~J} / \mathrm{m}^{2}$, and (b) SEM imaging of a copper target irradiated with a $65 \mathrm{fs}$ single pulse at an absorbed laser fluence of $3500 \mathrm{~J} / \mathrm{m}^{2}[18,43]$. The computational and experimental results demonstrate the same characteristic surface features, such as nanoprotrusions, nanocavities, nanopores, and nanorims. The atoms in (a) are colored according to their elevation with respect to the initial surface.

Vorobyev and Guo in Ref. [43] explained the formation of these nanostructures on the surface of $\mathrm{Cu}$ target by the initial non-uniform distribution of the laser energy which results in the formation of localized nanoscale melted regions within the incident laser spot. The formation of the nanomelted regions causes high temperature gradients, which in turn leads to high surface tension gradients resulting in the expulsion of liquid to the adjacent colder regions. Eventually, the nanocavities, nanorims and nanoprotrusions are formed due to the fast cooling of the expelled liquid structures.

The surface morphology obtained in the TTM-MD simulation of a bulk (001) Cr target irradiated with a $50 \mathrm{fs}$ laser pulse at an absorbed laser fluence of $1100 \mathrm{~J} / \mathrm{m}^{2}$ is differ from the one obtained in earlier TTM-MD simulation of a bulk (001) Ag target irradiated with a 100 fs laser pulse at an absorbed laser fluence of $900 \mathrm{~J} / \mathrm{m}^{2}$, which is slightly above the threshold for transition from melting to photomechanical spallation, Ref. [59]. One of the main differences that can be distinguished very clearly between the two metals is the number of the solidified spikes that 
remain in the system after the complete solidification of the new surfaces. Even though the lateral size of the two computational systems is similar, $98.7 \mathrm{~nm} \times 98.7 \mathrm{~nm}$ for $\mathrm{Ag}$ and $100 \mathrm{~nm} \times$ $100 \mathrm{~nm}$ for $\mathrm{Cr}$, nine solidified spikes are present on the $\mathrm{Cr}$ surface while just one spike is observed on the surface of Ag target, as can be seen in Fig. 2.12(a) and (b). The difference in the surface nanostructures generated on the two metal targets may be related to the difference in the surface tension of liquid $\mathrm{Cr}$ and $\mathrm{Ag}$, as well as the difference in the depth of melting of the irradiated targets at the spallation thresholds. The surface tension of pure silver measured in sessile-drops experiments for a temperature range from 1253 to $1373 \mathrm{~K}$ is found to be $\sim 890 \mathrm{mN}$ $\mathrm{m}^{-1}$ [175], Fig. 2. 12 (c). At the same time, surface tension measured for pure chromium for temperatures ranging between 1920 and $2218 \mathrm{~K}$ using the levitated droplet method is found to be $1780 \pm 40 \mathrm{mN} \mathrm{m}^{-1}$ at the melting temperature [176], Fig. 2.12(d). Accordingly, the high surface tension in $\mathrm{Cr}$ at the melting temperature facilitates the breakdown of the transient liquid structures and helps to create smaller nanostructures captured by the solidification process. This process proceeds differently in the case of $\mathrm{Ag}$, where the low surface tension at melting temperature allows for a larger extension and coalescence of liquid bridges into a single spike during the movement of the spalled liquid layer away from the bulk target. Furthermore, the larger depth of melting in the case of $\mathrm{Ag}, \sim 80 \mathrm{~nm}$ versus $36 \mathrm{~nm}$ for $\mathrm{Cr}$, contribute to the growth of voids with large diameters, which results in the formation of coarser surface structures. 

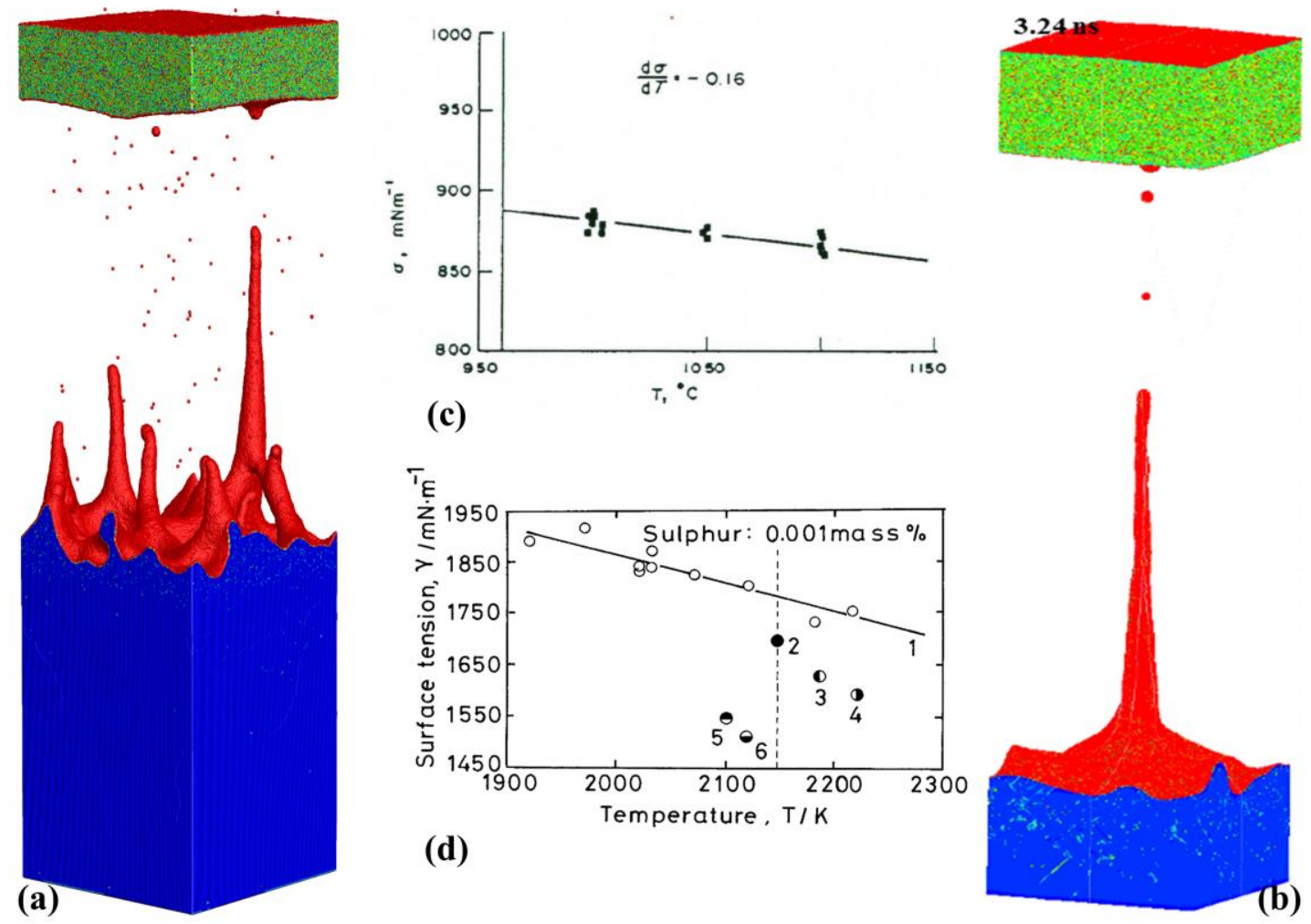

(c)

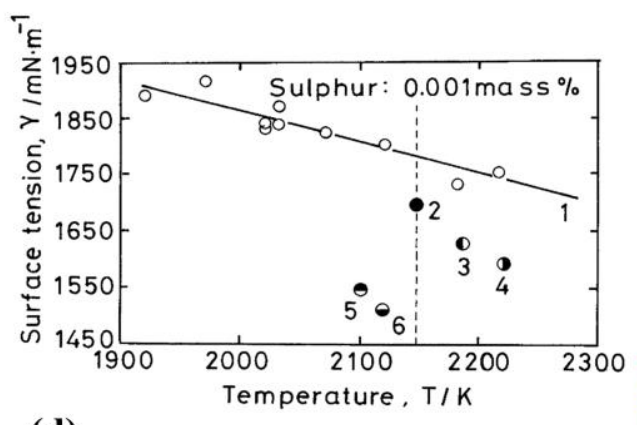

(d)

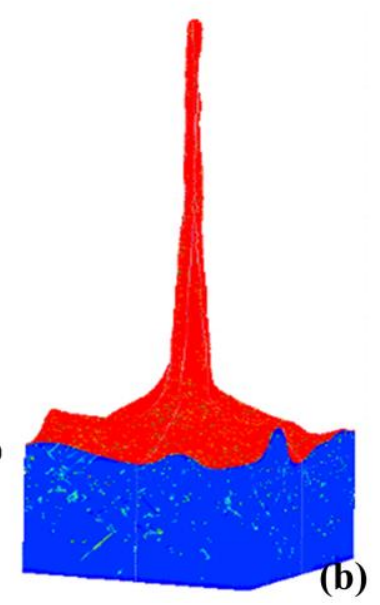

Fig. 2.12. Snapshots of atomic configurations obtained in TTM-MD simulations of (a) a bulk (001) $\mathrm{Cr}$ target irradiated with a $50 \mathrm{fs}$ laser pulse at an absorbed laser fluence of $1100 \mathrm{~J} / \mathrm{m}^{2}$, and (b) a bulk (001) $\mathrm{Ag}$ target irradiated with a $100 \mathrm{fs}$ laser pulse at an absorbed laser fluence of $900 \mathrm{~J} / \mathrm{m}^{2}$ [59]. The snapshots are taken after complete spallation of liquid layers and solidification of the surface regions of the targets. The formation of nine spikes in $\mathrm{Cr}$, as compared to only one spike on the Ag targets, may be attributed to the differences in the surface tension between the two metals. The surface tension of liquid Ag at the melting temperature is $\sim 890 \mathrm{mN} \mathrm{m}^{-1}$ (c) [175], which is almost twice smaller than the one in pure Cr system, 1780 $\mathrm{mN} \mathrm{m}^{-1}(\mathrm{~d})[176]$.

\subsection{Summary}

A series of simulations of femtosecond laser interactions with $\mathrm{Cr}$ targets is performed with a hybrid computational model that combines the atomistic MD method with the continuum TTM model. The results from small scale simulations reveal the microscopic mechanisms of laser-induced melting and solidification of the surface region of the target, photomechanical spallation, and explosive disintegration and ejection of a superheated surface region. The physical conditions that result in the transitions from the melting regime to spallation and from spallation to the regime of phase explosion are discussed based on the results of the simulations. The ejection of the liquid layers and large droplets in the spallation regime are found to provide 
an energetically-efficient way to remove material from the irradiated target. The transition from the spallation to the phase explosion regime, on the other hand, reduces the ablation efficiency and is characterized by a remarkable change in the composition of the ablation plume (smaller droplets and larger fraction of vapor phase). The timescales of melting and solidification are also affected by the dominant mechanism of the material ejection: the onset of spallation interrupts the heat conduction from the hot surface layer separated from the bulk the target, leading to a sharp drop in the maximum melting depth and the duration of the melting process, whereas the transition to the phase explosion regime increases the melting depth and the duration of the melting and solidification cycle due to the continued interaction of the ablation plume with the surface of the target. The predictions of the simulations are related to the experimental observations and the results of earlier MD simulations of laser interactions with other metal targets and molecular systems.

The analysis of the results obtained from a large-scale simulation of femtosecond laser pulse interactions with (001) Cr target provides microscopic insights into the mechanisms responsible for the photomechanical spallation of a liquid layer from the irradiated target. The relaxation of the strong compressive stresses generated by the rapid laser energy deposition leads to the formation of an unloading tensile wave that propagates from the free surface to the bulk of the target. At a certain depth under the surface, the tensile stresses exceed the dynamic strength of the melted material and causes nucleation of multiple voids in a sub-surface region of the target. The subsequent growth, coalescence, and percolation of voids results in the formation of a transient foamy structure of interconnected liquid regions that connect the upper liquid layer with the bulk of the target. Eventually, the liquid bridges breakup and the molten layer separates from the target. Shortly after the separation of the liquid layer, the liquid surface structures are captured by the advancing solidification front, leading to the formation of complex surface morphology, similar to the ones observed experimentally on metal surfaces irradiated by femtosecond laser pulses. 


\section{Chapter 3}

\section{Generation of crystal defects in different regimes of laser interactions with metals}




\subsection{Introduction}

The fast rate of energy deposition in short pulse laser processing applications may not only induce the rapid melting and resolidification of a surface region of an irradiated target, but can also result in the generation of strong compressive stresses, as discussed above in Chapter 2. The relaxation of the laser-induced stresses, taking place simultaneously with melting and resolidification of the surface region of the irradiated target, can result in the generation of a high density of crystal defects (vacancies, interstitials, dislocations, grain boundaries) and corresponding modification of physical and mechanical properties of the surface layer.

Small size of the laser-modified region (on the order 100s of $\mathrm{nm}$ ) in femtosecond and picosecond laser processing of metals enables, with appropriate design of boundary conditions, the direct MD modeling of the laser-induced generation of crystal defects. Indeed, the emission of partial dislocations from the melting front [121, 177], plastic deformation of films heated by a laser pulse [178], generation of nanocrystalline structure [179] and dislocations [180, 181] around the regions of localized laser energy deposition, strong supersaturation of a surface layer with vacancies [129], and transformation of a lattice-mismatched interface into a threedimensional periodic array of stacking fault pyramids outlined by stair-rod partial dislocations [141] have been observed in MD simulations.

In this section, we used atomistic modeling to: (1) investigate the generation of point defects in different regimes of laser-metal interactions and (2) to reveal the mechanisms responsible for the generation of dislocations in bcc $\mathrm{Cr}$ targets. The parameters of the computational model are briefly outlined in Section 3.2. The detailed structural analysis of the atomic configurations obtained in simulations of (001) $\mathrm{Cr}$ and $\mathrm{Ni}$ targets irradiated by short laser pulses is presented in Section 3.3 and used to elucidate the mechanisms responsible for the generation of point defects (mainly vacancies) that remain in the metal targets after resolidification of the transiently melted surface regions. The laser fluence dependence of the concentration of vacancies generated in the surface regions of irradiated targets is investigated in Section 3.4. Finally, in section 3.4, the analysis of the results obtained in four large-scale TTMMD simulations of (001) $\mathrm{Cr}$, (110) $\mathrm{Cr}$, and (111) Cr targets irradiated by 50 fs laser pulse is 
provided and the dependence of the dislocation emission on the crystallographic orientation of Cr target is discussed.

\subsection{Computational model}

The description of the dimensions of the computational cells and parameters of the interatomic interactions used in the simulations of laser interactions with $\mathrm{Cr}$ and $\mathrm{Ni}$ targets is provided below.

\subsubsection{Computational setup for simulations of laser interactions with $\mathrm{Cr}$ targets}

Three large-scale TTM-MD simulations of (001), (110), and (111) Cr targets irradiated by a $50 \mathrm{fs}$ laser pulse at an absorbed laser fluence of $800 \mathrm{~J} / \mathrm{m}^{2}$ are performed. The detailed description of the dimensions of the computational cell for $\mathrm{Cr}$ target in small and large-scale simulations, the parameters of the interatomic interaction in the MD part of the model, and the parameters that are used in the TTM equation is provided in Section 2.2.

\subsubsection{Computational setup for simulations of laser interactions with Ni targets}

A series of TTM-MD simulations of short pulse laser interactions with Ni target was performed earlier with the purpose of investigating the material response to the laser excitation in the three main irradiation regimes corresponding to the melting and resolidification of the surface region of the target, photomechanical spallation of a single or multiple layer(s), and ablation driven by the thermodynamic driving forces. The results of these simulations were published in Ref. [119]. In order to bring to light the generation of the crystal defects in the regions that experienced transient melting and solidification, an additional structural analysis of the final configurations generated in these simulations is performed and the results of this analysis are reported in Sections 3.3 and 3.4.

The MD part of the TTM-MD model for Ni targets represents the top $100 \mathrm{~nm}$ from the surface in the low laser fluence regime (below the threshold for laser spallation), and $200 \mathrm{~nm}$ at higher laser fluences (in the spallation and phase explosion regimes). The lateral dimensions of the MD computational cell are $3.35 \times 3.35 \mathrm{~nm}^{2}$, with the number of atoms equal to 113600 for the $100 \mathrm{~nm}$ and 227200 atoms for the $200 \mathrm{~nm}$ systems [119]. In the continuum part, the diffusion equation for the electron temperature is solved down to $1 \mu \mathrm{m}$, providing a sufficient 
representation of the electron heat conduction into the bulk of the metal target. The interatomic interaction in the MD part for the $\mathrm{Ni}$ target is described by the EAM potential in the form proposed in Ref. [182]. This EAM potential is fitted to reproduce some of the basic characteristics of $\mathrm{Ni}$ crystals, such as the lattice constant, elastic moduli, vacancy formation energy, and sublimation energy. The parameters used in the TTM equation for the electron temperature are listed below. The electron heat capacity is $C_{e}=\gamma T_{e}$ with $\gamma=1065 \mathrm{Jm}^{-3} \mathrm{~K}^{-2}$, the thermal conductivity is described by $K_{e}=K_{0} \frac{T_{e}}{T_{l}}$ with $K_{0}=91 \mathrm{Wm}^{-1} \mathrm{~K}^{-1}$, the electron-phonon coupling factor is $G=3.6 \times 10^{17} \mathrm{Wm}^{-3} \mathrm{~K}^{-1}$, and the optical absorption depth is $L_{P}=13.5 \mathrm{~nm}$. The target is irradiated by 1 ps laser pulse and, similar to the $\mathrm{Cr}$ simulations, the absorbed laser fluence rather than the incident fluence is used in the discussion of the results. The computational system is equilibrated at $300 \mathrm{~K}$ before applying the laser irradiation.

\subsection{Generation of point defects below the spallation threshold}

The laser energy deposition in the surface region of the metal target induces a rapid increase in the lattice temperature and buildup a strong compressive stresses in the surface region of the irradiated target, as has been discussed in Chapter 2. The relaxation of the compressive stresses in the presence of the free surface of the target leads to the generation of an unloading tensile stress wave that propagates deeper into the bulk target. The high lattice temperature along with the generation of compressive and tensile stresses causes melting of a surface layer of the target. The fast electron heat conduction that transfers the thermal energy deeper into the bulk of the system, along with the expansion of the surface region of the metal target, cause rapid cooling of the melted region. As soon as the temperature near the liquid-solid interface drops below the melting temperature of the target material, the melting turns into epitaxial resolidification.

The detailed structural analysis for the results obtained in the TTM-MD simulations for the $\mathrm{Cr}$ and $\mathrm{Ni}$ targets reveals the formation of a high density of crystal defects which may have a significant effect on the physical, chemical, and mechanical properties of the surface regions of the metal targets. The analysis of the atomic configurations for the $\mathrm{Cr}$ target irradiated at 850 $\mathrm{J} / \mathrm{m}^{2}$, which is $70 \%$ above the threshold for surface melting and just below the threshold for spallation, reveals the formation of a high density of point defects and a transient formation of 
planar defects. While the latter are discussed in Section 3.5.1, in this section we focus our attention on point defects. In order to provide a clear view for the crystal defects, several atomic configurations generated in the simulations are quenched for 1 ps using the velocity damping technique and shown in Fig. 3.1(a). In each snapshot, all the atoms with low potential energy that have local surroundings of the original bcc crystal structure are blanked, and only the atoms with high potential energy, which belong to the crystal defects or liquid phase, are shown.

The results for the Cr target, Fig. 3.1(a), illustrate the formation of a high density of vacancies generated by the laser irradiation of the target. Each vacancy in the snapshots is represented by a cluster of 14 atoms, which includes the first eight nearest neighbors and the second six nearest neighbors surrounding the lattice site of the missing atom. One self-interstitial defect is detected at $37 \mathrm{~nm}$ below the free surface. The interstitial adopts <110> dumbbell configuration and appears as a planar cross-like configuration oriented in one of the $\{110\}$ planes, with two red atoms in the middle. Several theoretical studies of metals with bcc crystal structure and interatomic interactions represented by either pair-wise [183] or EAM [184, 129] potentials demonstrate the ability of the interstitial to inhabit a $<110\rangle$ dumbbell configuration. The low density of self-interstitials, as compared to the high density of vacancies, is related to: (1) a high energy of self-interstitial formation, which is $~ 2-5$ times larger than the energy of vacancy formation, and (2) the high mobility of self-interstitial that allows them to diffuse quickly either to the liquid phase or to the free surface. The movement of the individual selfinterstitial, which is found in this simulation, can be observed in Fig. 3.1(a), where the interstitial changes its position and orientation during the period of $120 \mathrm{ps}$ for which the snapshots are shown in the figure. At the same time, the vacancies remain almost static in the metal target.

The total number of individual vacancies distributed in the top $38 \mathrm{~nm}$ surface layer of the $\mathrm{Cr}$ target is, 676 vacancies, which corresponds to a very high vacancy concentration of $0.47 \%$. In addition, eleven di-vacancies and six tri-vacancies are also observed between $2 \mathrm{~nm}$ and $16 \mathrm{~nm}$ below the surface. The strong temperature gradient created in the surface region of the target by the short-pulse laser irradiation combined with the rapid velocity of solidification front, which reaches a maximum value of $80 \mathrm{~m} / \mathrm{s}$ by the time when the melting front reaches the surface of the target, both provide exceptional conditions for stabilization of the highly non-equilibrium vacancy concentration in the surface region of the target. 
Fig. 3.1. Snapshots of atomic configurations obtained in TTM-MD simulations of (a) bulk EAM Cr target irradiated with 200 fs laser pulse at an absorbed laser fluence of $850 \mathrm{~J} / \mathrm{m}^{2}$, and (b) bulk EAM Ni target irradiated with 1 ps laser pulse at an absorbed laser fluence of $645 \mathrm{~J} / \mathrm{m}^{2}$. The two laser fluences correspond to the regime of melting and resolidification for the two targets.

The snapshots are quenched for 1 ps in order to reduce the thermal noise in the atomic positions and energies. The atoms are colored according to their potential energies. All the atoms that belong to bcc and fcc crystal structure in the $\mathrm{Cr}$ and $\mathrm{Ni}$ targets and have low potential energy are blanked, and only atoms with potential energy higher than $-3.99 \mathrm{eV}$ for EAM $\mathrm{Cr}$ and $-4.35 \mathrm{eV}$ for EAM Ni are shown. These atoms correspond either to the liquid phase or to the point defects that remain in the system during the solidification process.

The snapshots are taken along the [010] view direction down to the depth of 38 $\mathrm{nm}$ for $\mathrm{Cr}$ and $19 \mathrm{~nm}$ for $\mathrm{Ni}$ targets from the level of the initial surface. The defect configurations labeled as "A" are vacancies, and " $\mathrm{B}$ " is a self-interstitial, while "C" marks a cluster of 25 vacancies that are generated in the $\mathrm{Ni}$ target during the solidification process.
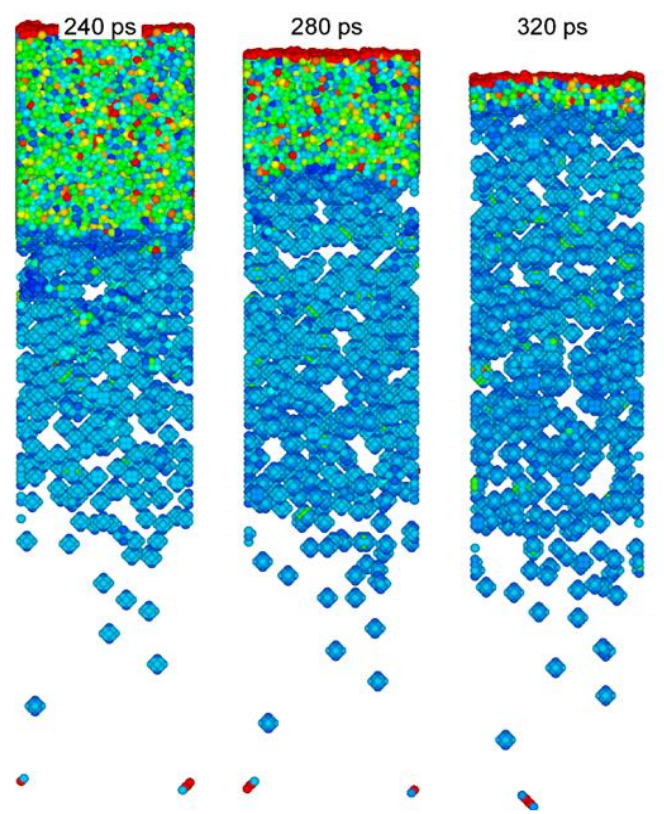

$360 \mathrm{ps}$

(a)
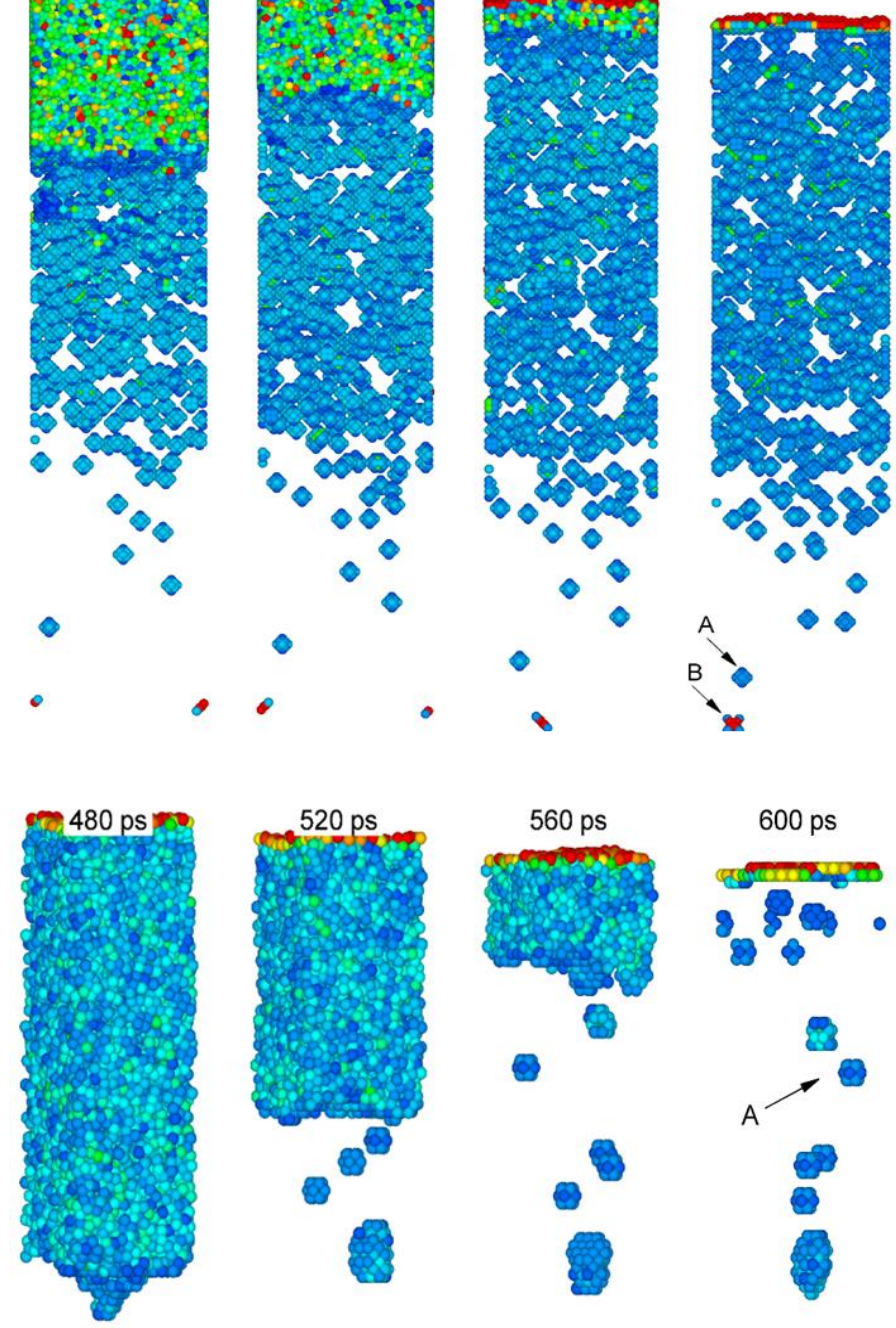

(b)

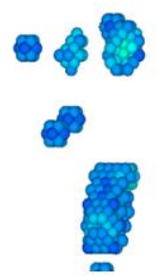

$600 \mathrm{ps}$
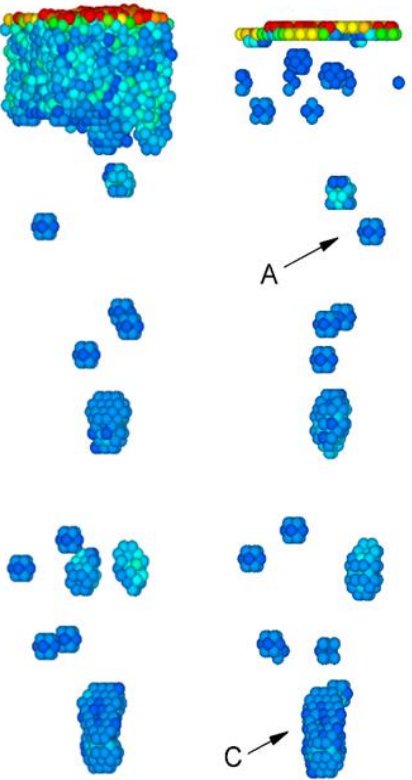
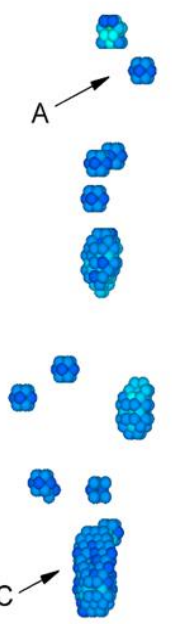
The concentration of point defects is also investigated for fcc Ni fcc targets is irradiated by short laser pulses at a broad range of absorbed laser fluences [119], in order to compare the concentration of vacancies/interstitials in two metals with different crystal structures. Fig. 3.1(b) shows snapshots of the atomic configurations that are obtained in a TTM-MD simulation for $\mathrm{Ni}$ target irradiated by 1 ps laser pulse at an absorbed laser fluence of $645 \mathrm{~J} / \mathrm{m}^{2}$, which is about $30 \%$ above the threshold for surface melting. The same visualization technique that is applied to the bcc $\mathrm{Cr}$ target is also used for the fcc Ni target. The results show the generation of high density of point defects in the surface region of the target. The mechanism responsible for the generation of vacancies is mostly the same as discussed above for $\mathrm{Cr}$, and leads to the formation of 19 vacancies in the top $32 \mathrm{~nm}$ of the Ni target. In this simulation, not a single self-interstitial is observed in the system at the end of the simulation, and the generation of point defects is limited to the formation of vacancies in the top region of the target. In the representation used in Fig. 3.1(b), each individual vacancy is represented by a cluster of 12 atoms which are the first nearest neighbors surrounding the vacant lattice site. In addition to the individual vacancies, we detected the formation of six clusters of vacancies each consisting of 5, 10, 12,12, 13, and 25 individual vacancies. These clusters of vacancies are oriented along the epitaxial growth direction, and they are located between $6.3 \mathrm{~nm}$ and $27 \mathrm{~nm}$ below the surface. The formation of clusters of vacancies in the fcc metal target has been observed experimentally in samples treated by various processing techniques such as, quenching from high temperature, electron irradiation within high-voltage electron microscope, fission and fusion neutron irradiation, and plastic deformation [185, 186].

In both bcc $\mathrm{Cr}$ and fcc $\mathrm{Ni}$ targets, the high densities of vacancies are found in the regions that had experienced transient melting and solidification processes. The distribution of the vacancies with respect to the depth under irradiated surface, presented in Fig. 3.2, confirms that a large fraction of the vacancies in bcc $\mathrm{Cr}$ and all of the vacancies in fcc $\mathrm{Ni}$ are located in the solidified part of the surface region of the target. The vacancy concentrations in these regions are very high $(0.47 \%$ for $\mathrm{Cr}$ and $0.25 \%$ for $\mathrm{Ni})$ as compared to the equilibrium concentrations of vacancies at the melting temperature $(0.0032 \%$ for $\mathrm{Cr}$ and $0.000111 \%$ for $\mathrm{Ni})$. Moreover, the concentration of vacancies in $\mathrm{Cr}$ is about two times higher than the one in the fcc Ni target. 

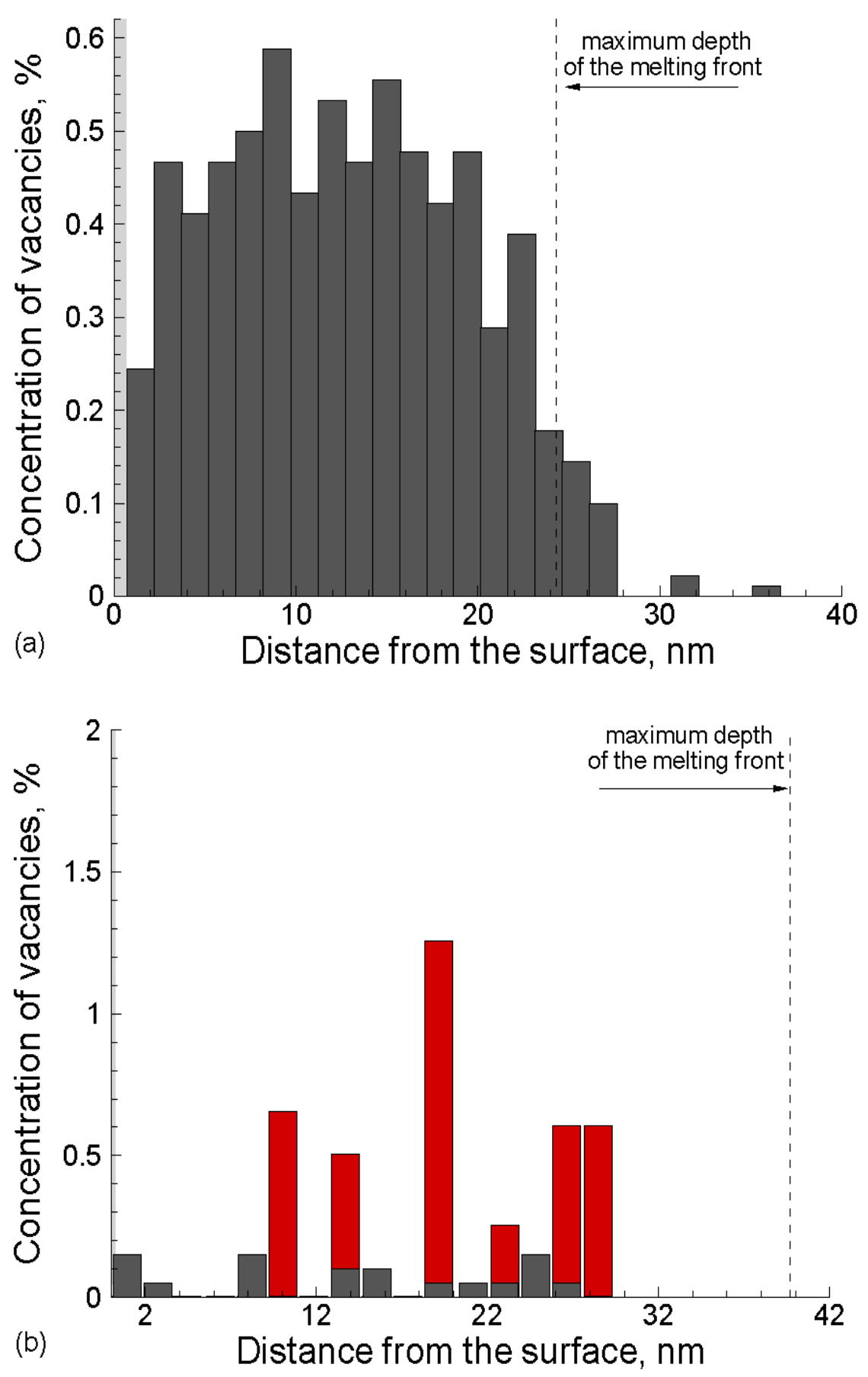

Fig. 3.2. Distribution of vacancies in the surface region of the bulk EAM Cr (a) and EAM Ni (b) targets irradiated by short laser pulses at laser fluencies above the threshold for surface melting. Snapshots of the atomic configurations used in the analysis are shown in Fig. 3.1. Each gray bar in the histograms is the result of the averaging over ten individual (001) atomic planes. The red bars in (b) represent contribution from the six clusters of vacancies that are detected in the Ni target. The dashed lines mark the depth of the region that experienced transient melting and solidification in response to the laser irradiation. 
The difference in the concentration of vacancies is mainly related to two factors: (1) the slight differences in the energies of the vacancy formation between the two metals - the energy of the vacancy formation normalized by the thermal energy the melting point, $E_{v}^{f} / k_{B} T_{m}$, is 10.3

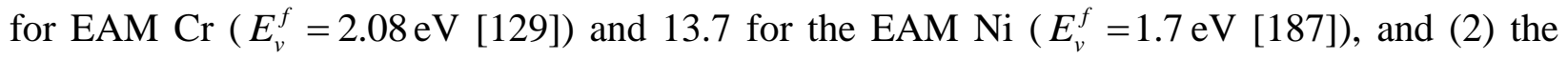
differences in the crystal structure between the close-packed fcc structure of $\mathrm{Ni}$ and a more open bcc structure of $\mathrm{Cr}$.

\subsection{Laser fluence dependence of the vacancy concentration}

We extend our analysis to the investigation of the effect of different laser fluences on the concentration of vacancies in the surface regions of $\mathrm{Cr}$ and $\mathrm{Ni}$ targets. The range of laser fluences considered in the simulations cover three distinct regimes of the material response to the laser irradiation: laser melting and solidification, photomechanical spallation, and phase explosion.

In EAM Cr, the concentration of vacancies ranges from $0 \%$ up to $\sim 0.47 \%$, as shown in Fig. 3.3. At absorbed laser fluence $426 \mathrm{~J} / \mathrm{m}^{2}$, the effect of laser energy deposition is limited to the formation of unstable stacking faults that appear within the top $23 \mathrm{~nm}$ layer below the surface in the first 10 ps. The formation of the stacking faults coincides with the propagation of the tensile component of the stress wave into the bulk target. These planar defects disappear as the tensile wave leaves the surface region of the target. More detailed discussion of the formation of planar defects in (001) $\mathrm{Cr}$ is presented in section 3.5. The maximum temperature of the surface in this simulation reaches $2100 \mathrm{~K}$, which is about $0.9 T_{m}$, and no melting or generation of point defects (vacancies or self-interstitial) is observed.

With the increase of the absorbed laser fluence to $638 \mathrm{~J} / \mathrm{m}^{2}$ and $850 \mathrm{~J} / \mathrm{m}^{2}$, which is above the melting threshold, the surface region of the target undergoes melting down to $\sim 3 \mathrm{~nm}$ and $\sim 30$ $\mathrm{nm}$, respectively [129, 134]. Accordingly, the complete solidification process of these surface layers yields vacancy concentration of approximately $0.153 \%$ at $638 \mathrm{~J} / \mathrm{m}^{2}$ and increases to $0.47 \%$ at $850 \mathrm{~J} / \mathrm{m}^{2}$. Meanwhile, the temperature of the surface at the end of the solidification process drops down $0.88 T_{m}$ for the low laser fluence and further reduces to $0.80 T_{m}$ when the fluence increases to $850 \mathrm{~J} / \mathrm{m}^{2}$. 

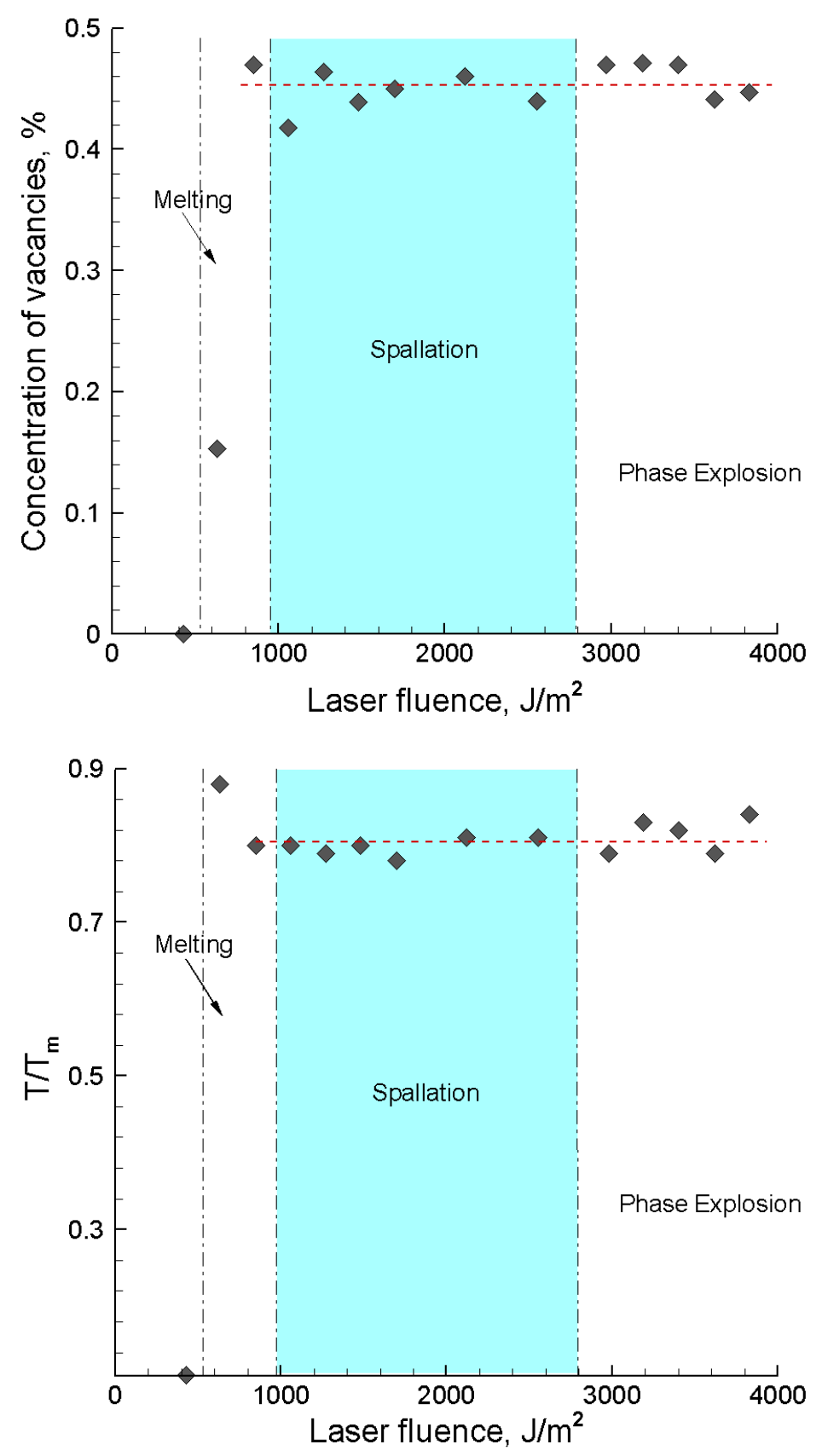

Fig. 3.3. Concentration of vacancies and the temperature of the surface region of the target at the end of the solidification process as functions of the absorbed laser fluence predicted in TTM-MD simulations of EAM Cr target irradiated by 200 fs laser pulse. The vertical dash-dotted lines mark the approximate values of the threshold fluences for the onset of laser melting, photomechanical spallation, and explosive decomposition of the surface region into vapor and liquid droplets (phase explosion). The range of fluences where the photomechanical spallation is the dominant mechanism of the material ejection is highlighted by the light blue color. The concentration of vacancies is averaged over for the region that has experienced transient melting and solidification. The red dashed lines show the mean of the data sets excluding the first two points (below and just above the melting threshold). 
The shallow depth of melting, $\sim 3 \mathrm{~nm}$, in the simulation performed at $638 \mathrm{~J} / \mathrm{m}^{2}$ can significantly contribute to the reduction of the concentration of vacancies, with the liquid phase serving as a sink for the defects.

As the irradiation conditions shift toward the photomechanical spallation regime (950 $\mathrm{J} / \mathrm{m}^{2}-2790 \mathrm{~J} / \mathrm{m}^{2}$ ), the magnitude of the tensile component of the laser-generated stress wave exceeds the dynamic strength of the material which causes the spallation or separation of one or multiple melted layers from the target [134]. The spallation of molten layer/layers interrupts the propagation of thermal energy from the surface to the bulk system and accelerates the solidification process in the remaining melted part of the $\mathrm{Cr}$ target. Although, a broad range of absorbed laser fluences, corresponding to the spallation regime has been tested for $\mathrm{Cr}$ target, the cooling rate, the velocity of the liquid-crystal interface, and the temperature of the surface region of the target at the end of the solidification process all remain within the same range. Accordingly, the solidification process in these simulations produced almost the same concentration of vacancies in the surface region of the target, between $\sim 0.42 \%$ and $0.47 \%$.

With further increase of the laser fluence above $2790 \mathrm{~J} / \mathrm{m}^{2}$, the temperature of the surface region of the target exceeds the threshold temperature for the onset of the phase explosion [134]. As mentioned earlier, in Section 2.3, the short pulse laser irradiation leads to overheating the surface region of the target and causes decomposition of the surface into a mixture of vapor atoms and liquid droplets. The ejection of numerous vapor atoms leads to the generation of a high vapor pressure above the surface of the target, an increase in the depth of melting, and deceleration of the solidification process. In spite of the increase in the laser fluence to very high values, of up to $3830 \mathrm{~J} / \mathrm{m}^{2}$, more than 6 time the melting threshold, the surface temperature at the end of the solidification process continue to be within the same range of $0.80 T_{m}-0.84 T_{m}$, which yields almost the same values of the vacancy concentration, ranging between $0.44 \%$ and $0.47 \%$.

Turning to the fcc Ni targets, the analysis of the vacancy concentration and the normalized surface temperature has been completed so far only for (1) melting and solidification, and (2) photomechanical spallation. The results from these simulations show that the concentration of vacancies for the EAM Ni target in these two regimes ranges from $0.034 \%$ to 0.058\%, as shown in Fig. 3.4. In the melting regime, the Ni target is exposed to absorbed laser fluences from $430 \mathrm{~J} / \mathrm{m}^{2}$ to $1720 \mathrm{~J} / \mathrm{m}^{2}$, the maximum depth of melting changes from $14 \mathrm{~nm}$ to 72 
$\mathrm{nm}$, and the solidification process produces concentrations of vacancies between $0.047 \%$ and $0.058 \%$. The high value of the concentration of vacancies, $\sim 0.08 \%$, observed in the simulation of $\mathrm{Ni}$ target at an absorbed laser fluence of $430 \mathrm{~J} / \mathrm{m}^{2}$ could be explained by the statistical fluctuation in the vacancy concentration value, Fig. 3.4.

The increase of the absorbed laser fluence up to $1930 \mathrm{~J} / \mathrm{m}^{2}$ leads to the transition to the photomechanical spallation regime for the EAM Ni target. This regime extends up to $3100 \mathrm{~J} / \mathrm{m}^{2}$. The magnitude of the tensile component of the stress wave in this regime becomes higher than the dynamic strength of the material, resulting in the spallation of one/multiple layers from the liquid region of the target. Meanwhile, the concentration of vacancies in the resolidified part of the target remains approximately within the same range, $0.036 \%-0.04 \%$, as does the temperature of the surface at the end of the solidification process, $0.81 T_{m}-0.86 T_{m}$. This is due to the interruption of the heat transfer from the hot spalled layers deeper into the target, which results in the rapid solidification process, as described in the discussion of the results for the $\mathrm{Cr}$ system in the photomechanical spallation regime.

In order to provide a clear physical understanding of the correlations between the undercooling temperature, velocity of solidification front and the vacancy concentration, a detailed analysis of the solidification process under well controlled temperature and pressure conditions is undertaken and is discussed in chapter 4. 

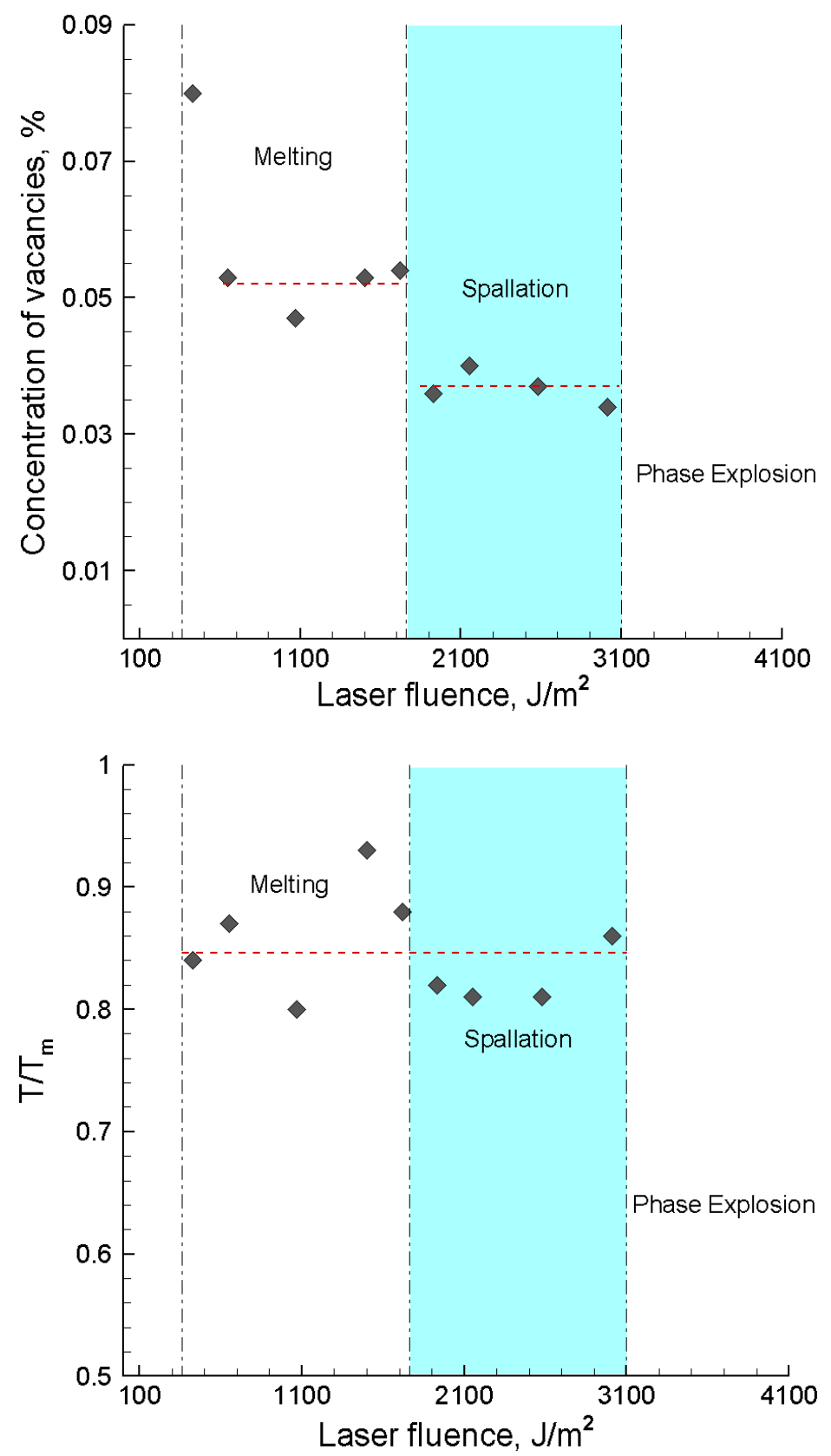

Fig. 3.4. Concentration of vacancies and the temperature of the surface region of the target at the end of the solidification process as functions of the absorbed laser fluence predicted in TTM-MD simulations of EAM Ni target irradiated by 1 ps laser pulse. The vertical dash-dotted lines mark the approximate values of the threshold fluences for the onset of laser melting, photomechanical spallation, and explosive decomposition of the surface region into vapor and liquid droplets (phase explosion). Due to the incomplete solidification process in the phase explosion regime, the results of the phase explosion regime are not shown in this figure. The range of fluences where the photomechanical spallation is the dominant mechanism of the material ejection is highlighted by the light blue color. The concentration of vacancies is average over the region that experienced transient melting and solidification. The red dashed lines show the mean of the data sets excluding the first point. 


\subsection{Generation of crystal defects in the interactions of short pulse laser with (001), (110) and (111) Cr targets}

With the rapid development in the efficiency of the computational modeling technology which involves, for example, the increase in the time and length scales accessible for the molecular dynamics simulations; it is now possible to simulate systems consisting of more than $10^{8}$ atoms, to increase the dimensions of the computational cell to several hundreds of nanometers, and to run the simulations for several nanoseconds. Indeed, opportunities for largescale MD modeling with highly efficient parallel computational codes have been successfully utilized to provide a solid understanding of the microscopic mechanisms responsible for the laser-induced melting, photomechanical spallation, and ablation of the metal targets in vacuum and under the conditions of spatial confinement [24, 188, 189].

While the detailed structural analysis of the laser-induced generation of point defects has been undertaken in small-scale MD simulations and the results provide a good understanding of the generation of vacancies in the surface regions of the irradiated targets in different irradiated regimes, the limitations on the maximum size of the systems accessible for MD simulations have limited our ability to investigate the evolution of other types of crystal defects. With the new parallel code and the increased availability of computational resources, three large-scale simulations of $\mathrm{Cr}$ targets with (001), (110), and (111) crystallographic surface orientation irradiated by a femtosecond laser pulse are performed. The goal of the simulations is to investigate the mechanisms responsible on the generation of dislocations in metal targets with bcc crystal structure and to reveal the dependence of the dislocation emission on the crystallographic orientation of the irradiated targets.

The microscopic mechanisms and kinetics of laser-induced melting in the surface region of the metal target have been discussed earlier in chapter 2. In this section, we only provide a brief overview the melting process occurring in the three systems in order to correlate it with the generation of the crystal defects in bulk $\mathrm{Cr}$ targets. Figure 3.5 shows contour plots of the temporal and spatial evolution of the lattice temperature and pressure in the surface region of (a) (001), (b) (110), and (c) (111) Cr targets. The three systems with dimensions of $100 \mathrm{~nm} \times 100$ $\mathrm{nm} \times 150 \mathrm{~nm}$ are exposed to a 50 fs laser pulse at an absorbed laser fluence of $800 \mathrm{~J} / \mathrm{m}^{2}$, which is $\sim 66 \%$ above the threshold for the transition to the melting and solidification regime. The contour 
plots illustrate the fast energy transfers from the excited electrons to the phonons resulting in a rapid increase of the lattice temperature above the melting temperature and causing prompt melting of 14, 26, and $23 \mathrm{~nm}$ thick top surface regions of (001), (110), and (111) Cr targets by the time of 11,18 , and $20 \mathrm{ps}$, respectively. As mentioned earlier in chapter 2 , the rapid increase in the lattice temperature in short-pulse laser processing takes place under the conditions of inertial stress confinement and results in the generation of strong compressive and tensile stresses in the surface region of the irradiated targets. Following the initial dynamic relaxation of the laser induced stresses that results in the propagation of a strong stress wave deeper into the bulk of the target, a quasi-static compressive stresses of with magnitude of $\sim 2$ GPa still remain in the crystalline part of the surface region of the target as can be seen from the pressure contour plots for the three systems in Fig. 3.5. The existence of the quasi-statistic compressive stress is mainly related to the large laser spot diameter, $\sim 100 \mu \mathrm{m}$, which only allows laser-induced thermoelastic stresses to relax by expansion in the direction normal to the surface. During the uniaxial expansion the stresses remain isotropic in the melted part of the target, however, the uniaxial expansion of the crystalline part results in anisotropic lattice deformation which corresponds to the anisotropic stresses. The anisotropic stresses cannot relax by the uniaxial expansion and the residual compressive stresses remain in the system long after the stress wave leaves the surface region, as can be seen in the pressure contour plot for the (001) $\mathrm{Cr}$ in Fig. 3.5(a). In contrast to the (001) $\mathrm{Cr}$ target, the residual compressive stresses in (110) and (111) $\mathrm{Cr}$ targets are partially relaxed by the emission of dislocations from the liquid-crystal interfaces. As the dislocations propagate deeper into the target, they reduces the residual compressive stresses, as can be seen in Figs. 3.5 (b) and (c). 

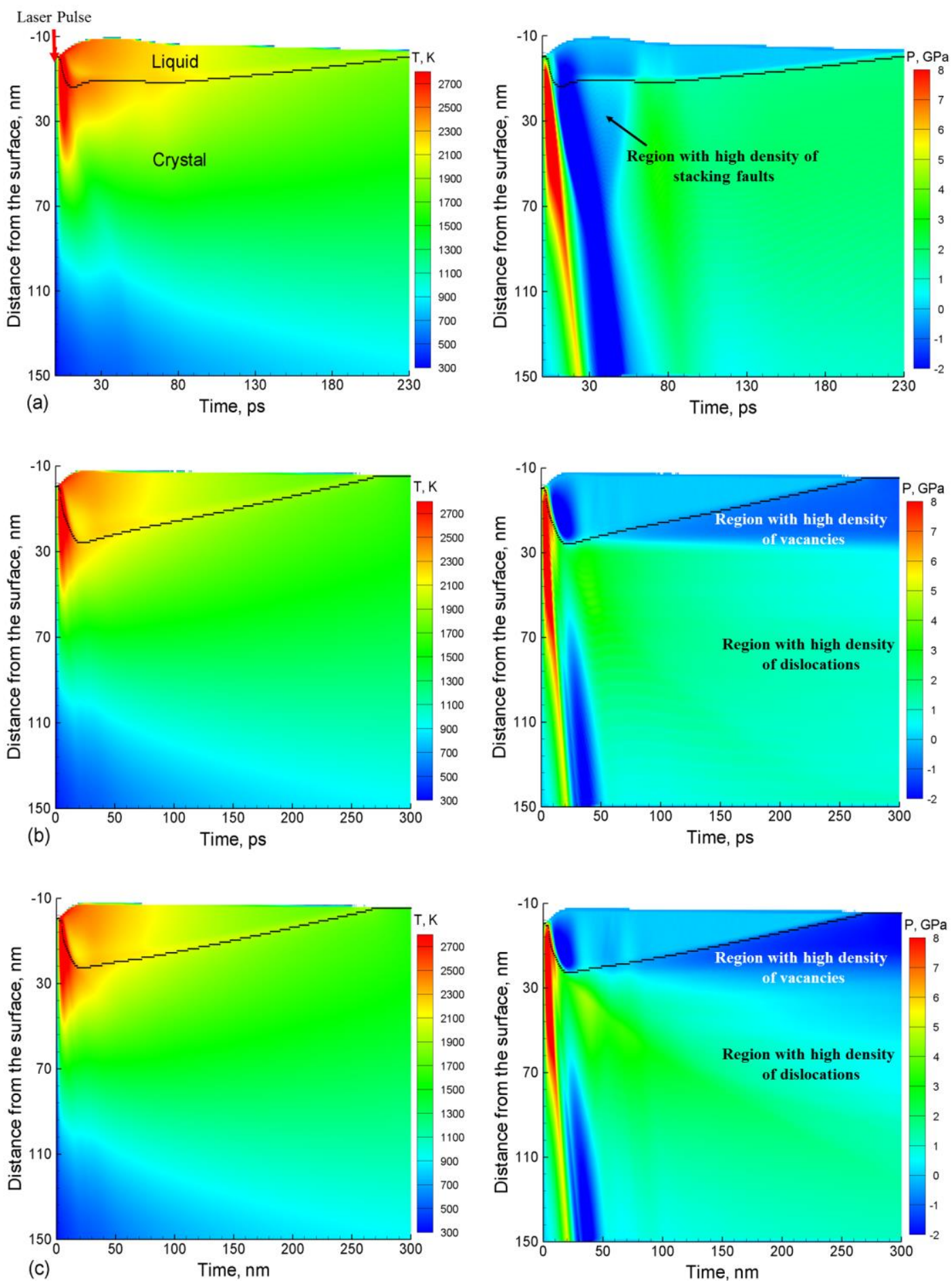

Fig. 3.5. Contour plots of the spatial and temporal evolution of the temperature (left) and pressure (right) in the simulations of bulk (a) (001) $\mathrm{Cr}$, (b) (110) $\mathrm{Cr}$, and (c) (111) Cr targets irradiated with a 50 fs laser pulse at an absorbed laser fluence of $800 \mathrm{~J} / \mathrm{m}^{2}$. The laser pulses are directed along the $Y$-axis from the top of the contour plots. Black lines separate the melted regions from the crystalline parts of the targets. 
The combined effects of the expansion of the hot surface region due to the relaxation of the laser-induced stresses and the fast electronic heat conduction that transfer the thermal energy deeper into the bulk target result in a rapid cooling of the surface region of the targets and causes rapid solidification of the transiently melted regions. The velocity of the solidification front increase with increasing undercooling and reaches $\sim 90 \mathrm{~m} / \mathrm{s}$ for the $(001), 130 \mathrm{~m} / \mathrm{s}$ for (110), and $115 \mathrm{~m} / \mathrm{s}$ for $(111) \mathrm{Cr}$ targets by the time when the melting fronts approach the surface of the targets and the surface temperature drops to $\sim 0.8 T_{m}$. The fast non-uniform solidification process leads to the formation of atomically-rough surface morphologies as represented in Fig. 3.6. To characterize the surface roughness of the targets, the surface is divided into square cells with dimensions of $0.25 \mathrm{~nm} \times 0.25 \mathrm{~nm}$ and the maximum elevation of atoms in each cell is determined and compared with other values along the surface. Ultimately, the highest and lowest elevation points are found for each surface and the standard deviation of the arithmetic mean of the elevations is calculated. The standard deviations are found to be $\sim 0.18 \mathrm{~nm}, 0.19 \mathrm{~nm}$, and 0.19 $\mathrm{nm}$, for (001), (110) and (111) Cr targets, respectively. Fig. 3.6 illustrates the surface topography of (001), (110), and (111) Cr targets at 270 ps after the laser pulse, when the solidification process is completed in all of the three simulations. The atoms in these maps are colored according their distance from the lowest elevation points which are considered to be zero.

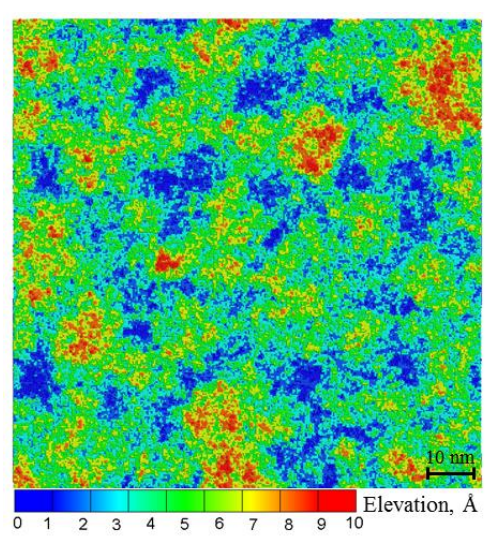

(001) $\mathrm{Cr}$

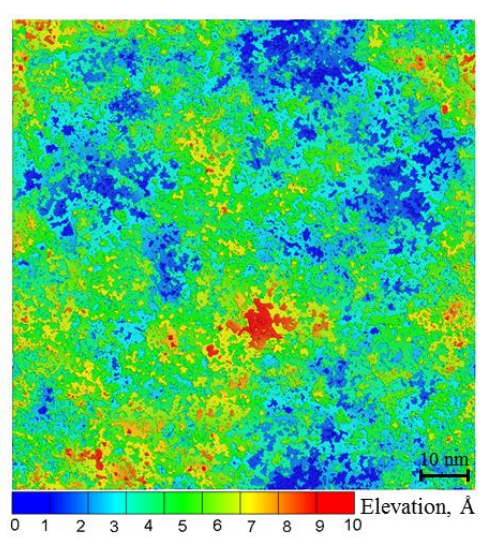

(110) $\mathrm{Cr}$

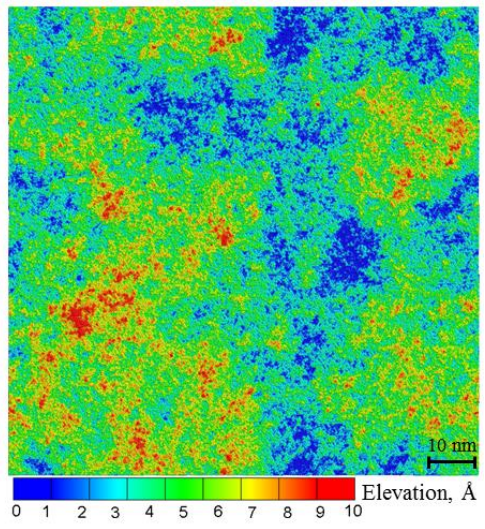

(111) $\mathrm{Cr}$

Fig. 3.6. Surface topographies of (001) $\mathrm{Cr}$, (110) $\mathrm{Cr}$, and (111) $\mathrm{Cr}$ targets irradiated by a 50 fs laser pulse at an absorbed of $800 \mathrm{~J} / \mathrm{m}^{2}$, which is $66 \%$ above the melting threshold. The surface topography is shown after complete solidification of the surfaces, for $270 \mathrm{ps}$ after the laser pulse. The atoms are colored according their distance from the lowest elevation points which are considered to be zero. 
The visual inspection of the snapshots of the atomic configurations for the three systems taken at different simulation times is used for the initial analysis of the laser-induced generation

of crystal defects in EAM Cr targets. This initial analysis reveals the formation of a high density of crystal defects in the surface regions of the metal targets. Below we provide a detailed structural characterization of the crystal defects that are formed in the three systems.

\subsubsection{Generation of partial and perfect dislocations in (001) $\mathrm{Cr}$ target}

Similar to the analysis of the results of laser-induced generation of crystal defects performed for atomic configurations obtained in the small-scale TTM-MD simulations of a femtosecond laser pulse interactions with $\mathrm{Cr}$ targets (see section 3.3), several snapshots of the atomic configurations produced in the large-scale TTM-MD simulation of a (001) $\mathrm{Cr}$ target irradiated by a 50 ps laser pulse are quenched using the velocity damping technique and shown in Fig. 3.7. The atoms in these snapshots are colored according to their potential energies and all the atoms that have energies below $-3.99 \mathrm{eV}$ and belong to the original bcc crystal structure are blanked to ensure a clear view of the crystal defects. 

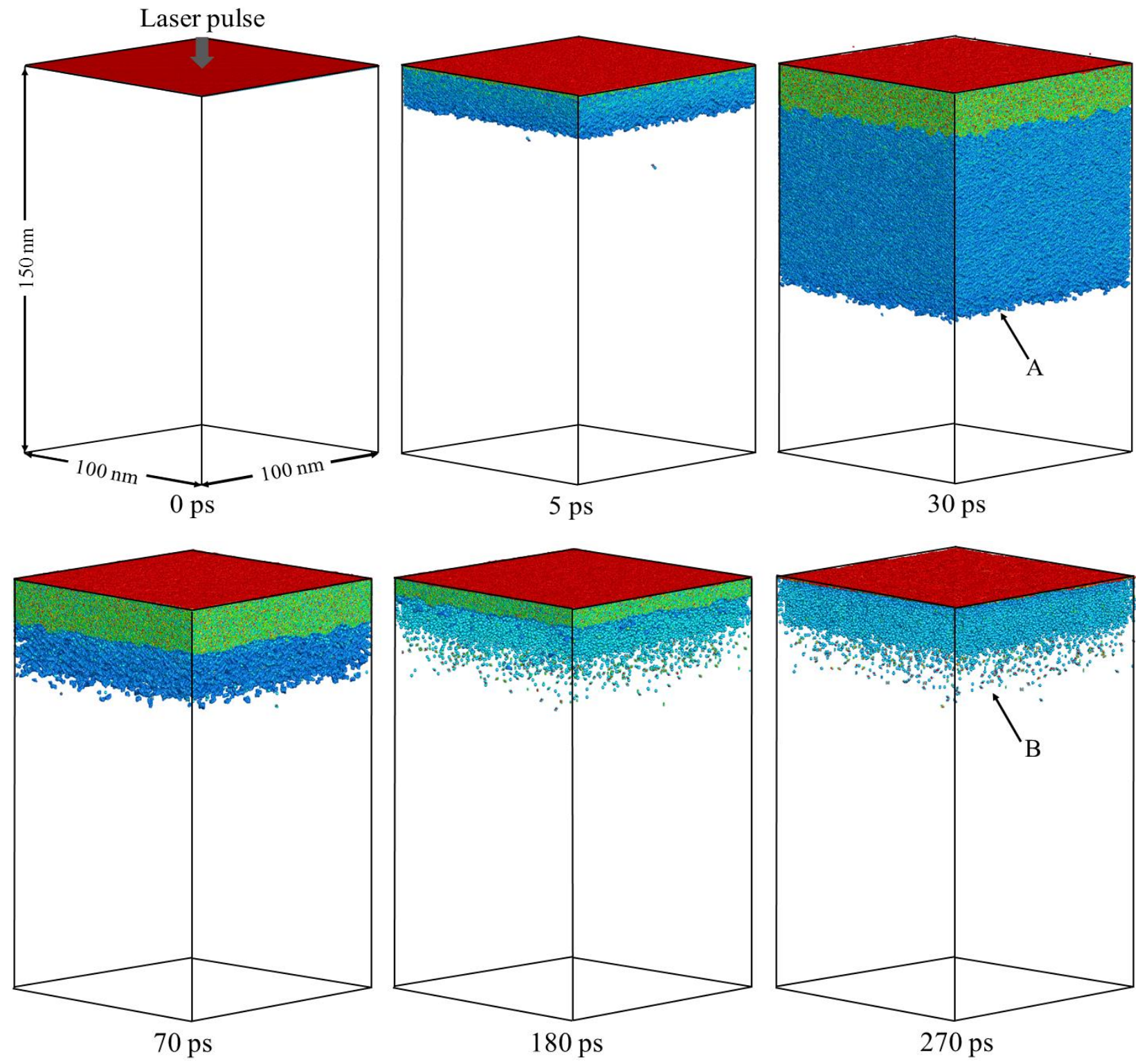

Fig. 3.7. Snapshots of atomic configurations obtained in a large-scale TTM-MD simulation of a bulk (001) $\mathrm{Cr}$ target irradiated with a 50 fs laser pulse at an absorbed laser fluence of $800 \mathrm{~J} / \mathrm{m}^{2}$, which is $66 \%$ above the threshold for surface melting. The snapshots are quenched to $0 \mathrm{~K}$ in order to reduce thermal noise in atomic positions and energies. Atoms are colored according to their potential energy and all the atoms that have energies below $-3.99 \mathrm{eV}$ and belong to the original bcc crystal structure are blanked to display the crystal defects. The red atoms in the top layer of the snapshots belong to the free surface of the target while the atoms in the green region (30, 70, and $180 \mathrm{ps)} \mathrm{represent} \mathrm{a} \mathrm{melted} \mathrm{region.} \mathrm{The} \mathrm{crystal}$ defects marked by $\mathrm{A}$ in a snapshot shown for 30 ps correspond to the stacking faults, while the ones marked by B in a snapshot shown for 270 ps are point defects, vacancies and self-interstitials, that remain in the top surface of the target after complete resolidification of the surface region. 
With this representation, we are only showing the atoms that belong to either the free surfaces (red atoms), the melted regions (green atoms), or the crystal defects (blue atoms). The visual inspection of the snapshots and the analysis of the pressure contour plot shown in Fig. 3.5(a) provide insights into the fast processes induced by the femtosecond laser irradiation. The pressure contour plot shows that rapid relaxation of the laser-induced stresses results in $2 \mathrm{~nm}$ surface expansion during the first 5 ps, which causes a reduction in the compressive stresses from $\sim 3 \mathrm{GPa}$ to $1 \mathrm{GPa}$ just below the irradiation surface. During this time, a complex pattern of stripes composed of atoms with elevated potential energy emerges directly below the surface region and propagates down to $10 \mathrm{~nm}$. As the unloading tensile stress wave propagated down from the free surface of the target, the region with the stripes follows the wave and reaches the depth of $42 \mathrm{~nm}$ by $10 \mathrm{ps}$ and the maximum depth of $85 \mathrm{~nm}$ by the time $30 \mathrm{ps}$. When the unloading wave leaves the surface region of the target, the expansion of the stripes turns into contraction until they completely disappeared by the time of $\sim 200$ ps after the laser pulse.

An enlarged view of the atomic configurations that correspond to the blue stripes is shown for a snapshot taken at 90 ps after the laser pulse in Fig. 3.8(a). The analysis of these configurations elucidates that these stripes consist of pairs of $\{011\}$ atomic planes tilted by about $\pm 45^{\circ}$ with respect to the [001] direction and shifted with respect to each other by a displacement vector of $a / 8<110\rangle$. The areas of the internal shifts correspond to the stacking faults outlined by partial dislocations with Burgers vectors equal to the displacement vectors. The transient appearance of the stacking faults has not been detected in experimental studies because of the short time for their existence. The unstable stacking faults in the bcc crystal structure, however, have been discussed theoretically, based on the hard-sphere model [190, 191, 192]. The analysis based on the hard-sphere model suggests that the stacking fault can be introduced in a bcc crystal if a part of the crystal along a $\{110\}$ plane is shifted by displacement vectors of a/ $8<110>$. Fig. 3.8(b) shows a schematic diagram of the atomic arrangement on a $(0 \overline{1} 1)$ plane of a bcc crystal structure, where the red circles represent the atoms in the top layer. It has been shown that the uniaxial expansion of the crystal can reduce the interatomic interaction beyond the first nearest neighbors, bringing the atoms to local energy minima and stabilizing the stacking faults. As first suggested by Steijen and Brick [193], the stacking faults may also appear during slip along a $<111>$ direction (e.g., [ $\overline{1} 11]$ direction shown by red dashed line in Fig. 3.8(b)), when a perfect 
dislocation dissociates into partial dislocations and follow <001> and <211> directions, e.g., $\frac{a}{2}[\overline{1} 11] \rightarrow \frac{a}{8}[011]+\frac{a}{4}[\overline{2} 11]+\frac{a}{8}[011]$, illustrated in Fig. 3.8(b).

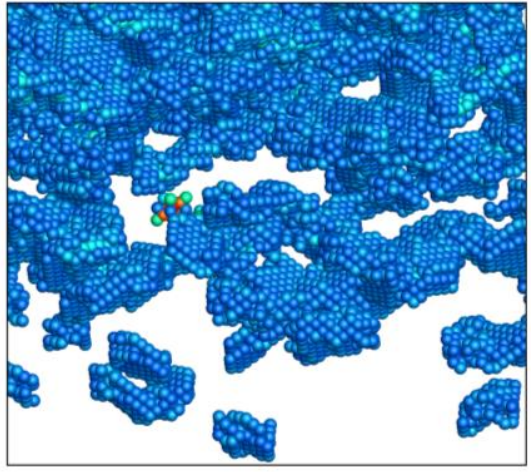

(a)

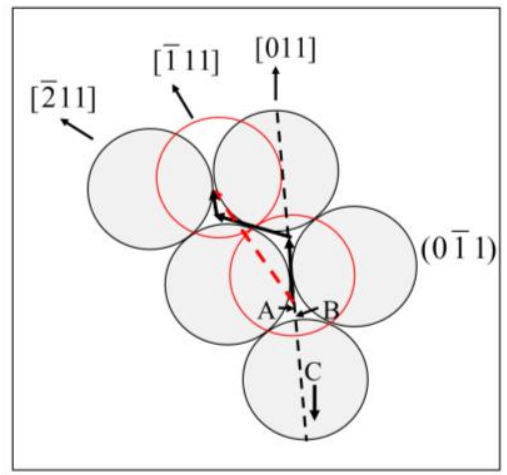

(b)

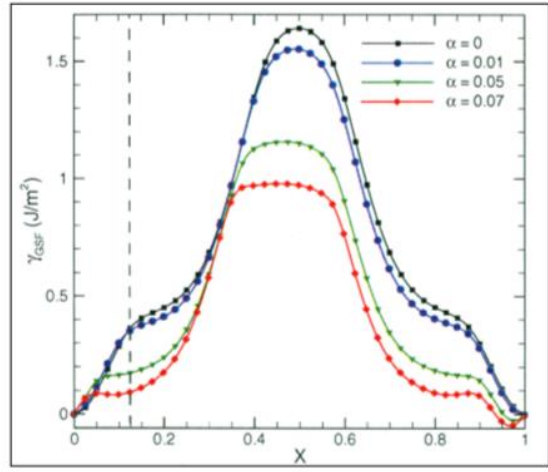

(c)

Fig. 3.8. (a) Close up view of the atomic configurations that correspond to the stacking faults observed in the TTM-MD simulation of (001) a Cr target irradiated by a $50 \mathrm{fs}$ laser pulse at an absorbed laser fluence of $800 \mathrm{~J} / \mathrm{m}^{2}$. The atoms are colored according to their potential energies and all the atoms that belong to the original bcc crystal structure and have a potential energy below $-3.99 \mathrm{eV}$ are blanked. The snapshot is taken at a time of $90 \mathrm{ps}$ after the laser pulse and shows that the stacking faults are represented by pairs of atomic planes oriented at $\pm 45^{\circ}$ with respect to the uniaxial expansion along [001] direction. The stacking faults appear in the system when a part of the crystal is shifted along $\{110\}$ planes by a displacement vectors of $\mathrm{a} / 8<011>$. The schematic diagram of the atomic arrangement on a $(0 \overline{1} 1)$ plane shown in (b), represent the prediction of the appearance of the stacking faults within the hard-sphere model, where the red circles represent the top layer. The possible formation of the stacking faults, from the hard-sphere model, is due to the dissociation of the perfect dislocation with a Burgers vector represented by the red dashed line along [ $\overline{1} 11]$ into [011] and [ $\overline{2} 11]$ displacements represented by the solid black arrows. The schematic diagram is taken from Ref. [190], the dashed black line along [011] marks the stacking faults at $X=1 / 8$, for the stacking faults that appear in the TTM-MD simulation. The diagram in (c) represent the result of the calculation of the generalized stacking faults energy, $\gamma_{\mathrm{GSF}}$, for the (100) slip plane in bcc EAM $\mathrm{Cr}$ as a function of the magnitude of fault vector, $\vec{u}=X \times[\overline{1}, 01+\alpha]$. The GSF curves are calculated for different values of the uniaxial strain, $\alpha$, applied to the bcc lattice in the [001] direction. The line marks the stacking faults at $X=1 / 8$, predicted with the hard-sphere model. The diagram in (c) is taken from Ref. [129].

The generation of unstable stacking faults have also been observed in earlier TTM-MD simulation of EAM Cr target irradiated with a 200 fs laser pulse at an absorbed laser fluence of $638 \mathrm{~J} / \mathrm{m}^{2}$ and the generalized stacking fault energy have been calculated for the EAM Cr material [129]. The results of this earlier work have demonstrated that the generation of unstable stacking 
faults in the EAM Cr target by a displacement in a $\langle 110\rangle$ direction along $\{110\}$ planes is associated with an increase of the uniaxial expansion from zero to 0.07 , which leads to the decrease the generalized stacking fault energy from $0.352 \mathrm{~J} / \mathrm{m}^{2}$ to $0.093 \mathrm{~J} / \mathrm{m}^{2}$ in the vicinity of $X=1 / 8$, where $X$ is the ratio of generalized stacking fault vector to the magnitude of a <011> vector. The results also show that the instability of the stacking faults is mainly related to the absence of the local minima in the GSF curves obtained for the strain-free lattice as shown in Fig. 3.8(c).

The results obtained from the hard-sphere model analysis and earlier MD simulations are consistent with the results of our large-scale simulation, where the appearance and propagation of the stacking faults between 3 and $30 \mathrm{ps}$ is directly correlated with the expansion of the surface region of the target due to the propagation of the unloading tensile wave. The tensile wave activates multiple shifts in the four out of six $\{110\}$ crystallographic planes oriented at $45^{\circ}$ with respect to the [001] direction of the material expansion. As mentioned earlier, the stacking faults on the $\{110\}$ planes promptly retract as the tensile stress wave leaves the surface region and the lattice deformation partially relaxes. The retraction of the stacking faults is also affected by an additional weaker unloading wave that appears between 90 and 120 ps and results in a small expansion of the stacking faults region before it continues to vanish and completely disappear by the time of $200 \mathrm{ps}$.

In addition to the transient formation of unstable planar defects in the course of the dynamic relaxation of laser-induced stresses, the melting and solidification of the top $14 \mathrm{~nm}$ results in the generation of a high density of point defects, mostly vacancies. More detailed discussion of the generation of point defects in large-scale simulations of $\mathrm{Cr}$ targets is provided in section 3.5.4. Although the generation of the point defects and stacking faults is observed very clearly in this simulation, the emission of perfect dislocations, is absent in this target. In order to explain the absence of the dislocation emission in this system, we recall the basic information about the slip systems in the body-centered cubic metals. The bcc crystal structure is not a closed-packed structure and the slip is found to occur in the closed-packed directions, $\langle 111\rangle$, on the crystallographic planes $\{110\},\{112\}$, and $\{123\}$ that contain the closed-packed directions. There are six $\{110\}$ slip planes each with two $\langle 111\rangle$ directions, twelve $\{112\}$ slip planes, and twenty-four $\{123\}$ slip planes with one $\langle 111\rangle$ direction each. Thus, in total there are 48 slip 
systems in the bcc crystal structure. Since the slip planes in the bcc structure are not closedpacked planes, it requires a high shear stress to cause the slip. The calculation of the resolved shear stress is conducted and explained in more details in section 3.5.3. In general, the results obtained from the shear stress calculations for the (001) $\mathrm{Cr}$ target indicate that the resolved shear stress below the liquid-solid interface remains below 1.23 GPa during the simulation time. This value is less than the half of the value of an estimate of the theoretical strength of a perfect $\mathrm{Cr}$ crystal (the theoretical critical shear stress in the absence of any dislocation), $\tau_{t h}=\frac{G}{30}=3.8 G P a$, where $G$ is the shear modulus equal to $115 \mathrm{GPa}$ for Cr, [194] [195], which indicates the difficulty in the generation of dislocations in this system. Our dependence on the theoretical value of the shear stress for $\mathrm{Cr}$ as a reference to compare the resolved shear stress is due to the fact that our sample is initially generated as a perfect sample, free from any crystal defects. At the same time, the generation of a strong stresses on the surface region of the target due to the rapid energy deposition of the laser pulse takes place within a very short time, which results in the rapid increase in the resolved shear stress to a value close to the theoretical values. For this reason, the resolved shear stress $1.23 \mathrm{GPa}$ is not enough to generate perfect dislocations in (001) Cr target.

In order to investigate the laser-induced generation of crystal defect in a $\mathrm{Cr}$ target at high absorbed laser fluence, we analyzed the results obtained from a large-scale TTM-MD simulation performed at an absorbed laser fluence of $1100 \mathrm{~J} / \mathrm{m}^{2}$, where the photomechanical spallation of a surface region of the target is observed are discussed above, in Section 2.3.3. A visual picture of the formation of crystal defects in the surface region of the target is provided by snapshots of the atomic configurations of the target shown in Fig. 3.9. The snapshots are taken at different simulation times and quenched to $0 \mathrm{~K}$ for $2 \mathrm{ps}$ using the velocity damping technique in order to reduce the thermal noise in the atomic positions and energies. This technique provides us with a clear view of the crystal defects that are generated in the system during the simulation and serves as a starting point in the analysis of the laser-induced generation of crystal defects. The atoms in these snapshots are colored according to their potential energies and all the atoms that belong to the original bcc crystal structure and have energies below $-3.99 \mathrm{eV}$ are blanked. Only the atoms that belong to the melted region (green region in the snapshots shown for 50, 150, and $250 \mathrm{ps)}$ ), free surface (red areas), or the crystal defects (blue atoms) are shown in these snapshots. The crystal defects marked by A, B, C, and D correspond to unstable stacking faults, point defects 
that include both vacancies and self-interstitials, perfect dislocations, and a dislocation that is formed due to the interaction of two dislocations, respectively.

Similar to lower-fluence TTM-MD simulation in the melting regime discussed above, the uniaxial expansion of the surface region of the target results in the transient appearance of a high density of the stacking faults, which completely disappear by the time of 250 ps after the laser pulse, as shown in Fig. 3.9. As it has been discussed in section 2.3.3, the rapid melting and resolidification of the top $36 \mathrm{~nm}$ surface layer of the target result in the generation of a high density of point defects, mostly vacancies, in the surface region of the target. In contrast to the lower-fluence simulation, however, the emission of perfect dislocations from the solidified surface is also detected in the simulation, starting from the first dislocation that is formed in the area marked by the black dashed square in Fig. 3.9 at a time of 250 ps, Fig. 3.9.

The combined effect of several factors may be responsible for the nucleation of the dislocation in the region outlined in Fig. 3.9. For instance: the results illustrate that the temperature at the depth where the dislocation emerged reaches $\sim 0.73 T_{m}$ at the time when the quasi-static compressive pressure approaches $\sim 2$ GPa. As has been mentioned earlier, the pressure is defined as $P=-\left(\sigma_{x x}+\sigma_{y y}+\sigma_{z z}\right) / 3$ and has contributions from the compressive stresses in the lateral directions, $\sigma_{x x}$ and $\sigma_{y y}$, where the lattice parameter is fixed at its room temperature value, $0.288 \mathrm{~nm}$, and the tensile stresses in the direction normal to the surface, $\sigma_{z z}$. The corresponding resolved shear stress at this time and depth reaches $\sim 1.4 \mathrm{GPa}$ for 12 slip systems, which may be sufficient to cause the emission of the dislocation. The other important factor that may play a role in the dislocation emission is the generation of high density of vacancies due to the raped advancement of the solidification front. 

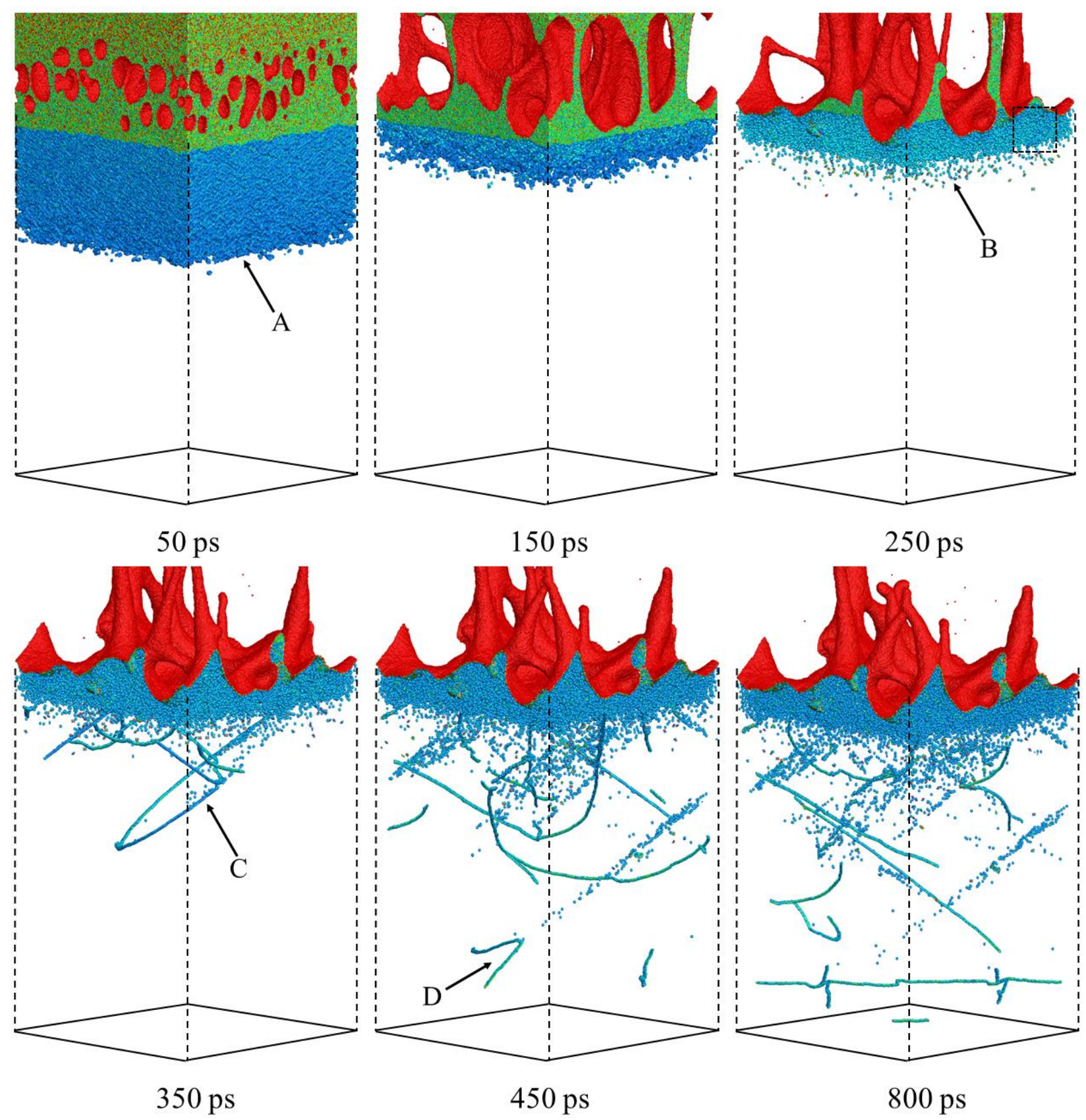

Fig. 3.9. Snapshots of the atomic configurations obtained in TTM-MD simulation of a bulk Cr target irradiated with a $50 \mathrm{fs}$ laser pulse at an absorbed laser fluence of $1100 \mathrm{~J} / \mathrm{m}^{2}$, slightly above the threshold of the photomechanical spallation. The configurations are quenched to $0 \mathrm{~K}$ for $2 \mathrm{ps}$ in order to reduce thermal noise from atomic positions and energies. The atoms are colored according to their potential energies, all the atoms that have energies below $-3.99 \mathrm{eV}$ are blanked and only the atoms that belong to either liquid phase (green), surface layer (red), or crystal defects (blue) are shown in the snapshots. The defects configuration marked by A, B, C, and D represent the stacking faults, vacancies and selfinterstitials, perfect dislocations, and a perfect dislocation resulting from the interaction of two dislocations in early time that partially annihilate and generate a tail of vacancies. The black dashed square in the snapshot shown for 250 ps represents the position the first dislocation is emitted. 
To provide a solid understanding of the nature of the dislocations emitted in this simulation, a detailed analysis of the defect structures is conducted with the help of the Dislocation Extraction Algorithm, DXA, where the dislocation lines are defined and the Burgers vectors are determined [196, 197, 198]. The results of the DXA analysis are given in Fig. 3.10 where the emission and propagation of the dislocations is presented and no other types of crystal defects are shown. Each point in the snapshots represents a dislocation segment and each segment is colored according to its Burgers vector. The blue color represents dislocation segments with $\frac{a}{2}\langle 111\rangle$ Burgers vector and while the light green color represents dislocation segments with $a\langle 100\rangle$ Burgers vector.

At a time of $250 \mathrm{ps}$, when the temperature near the resolidified surface drops to $0.73 T_{m}$, the compressive pressure approaches $\sim 1.8 \mathrm{GPa}$, and the resolved shear stress reaches $\sim 1.4 \mathrm{GPa}$ in twelve slip systems, the first perfect dislocations with Burgers vectors of $\frac{a}{2}\langle 111\rangle$ appear and start to propagate deeper into the target, Fig. 3.10. Beside the emission of several dislocations, we could follow the creation and annihilation of dislocations due to the interactions between the dislocations. For example, at a time of $400 \mathrm{ps,} \mathrm{which} \mathrm{is} \mathrm{not} \mathrm{shown} \mathrm{in} \mathrm{the} \mathrm{snapshots,} \mathrm{two}$ dislocations with Burgers vectors $\frac{a}{2}\langle 111\rangle$ and with opposite line senses are attracted to each other, combine, and annihilate the extended linear segments in each other, while the curved parts of the dislocations are connected together and marked by D in the snapshot shown for a time of 450 ps in Figs. 3.9 and 3.10. The annihilation of the linear parts of the two dislocations results in the formation of trail of vacancies that can be seen in the snapshot shown for a time of $450 \mathrm{ps}$ in Fig. 3.9. 


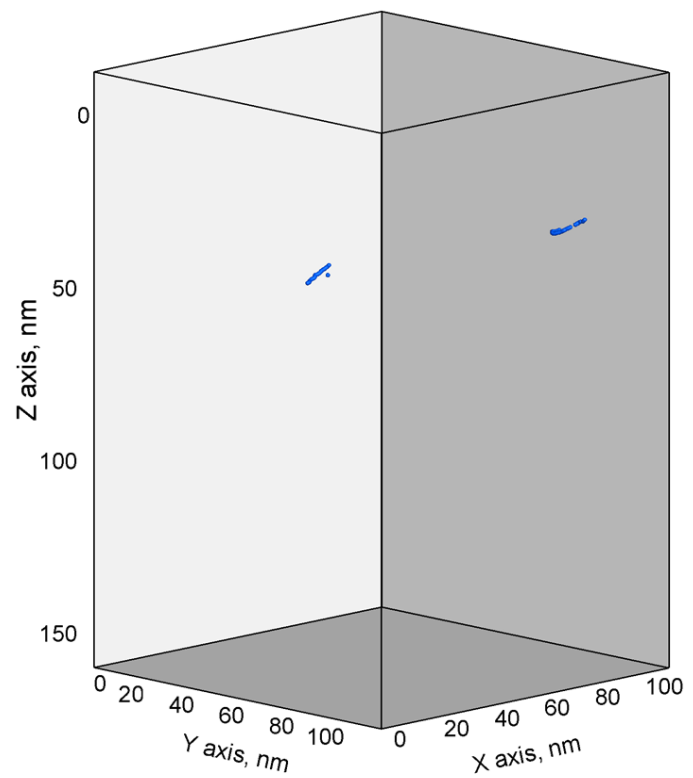

$250 \mathrm{ps}$

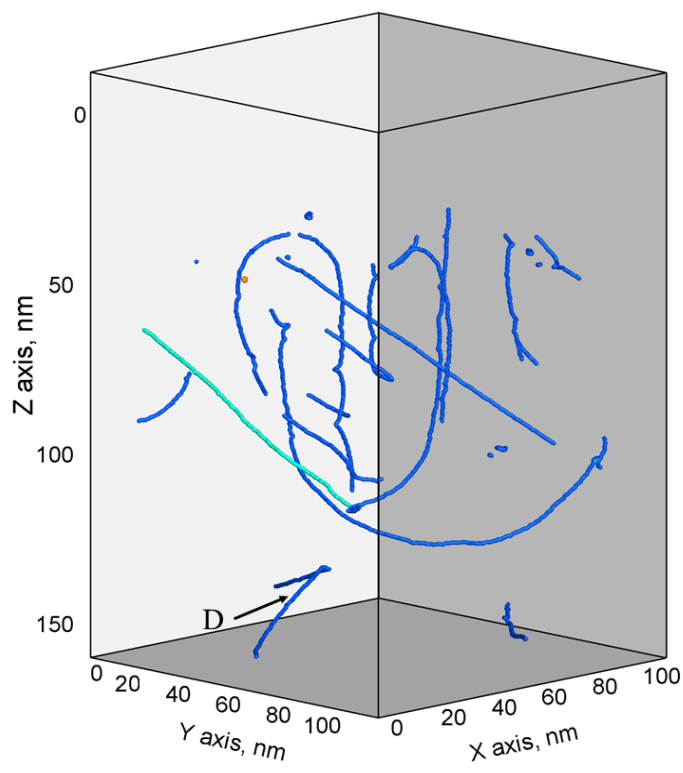

$450 \mathrm{ps}$

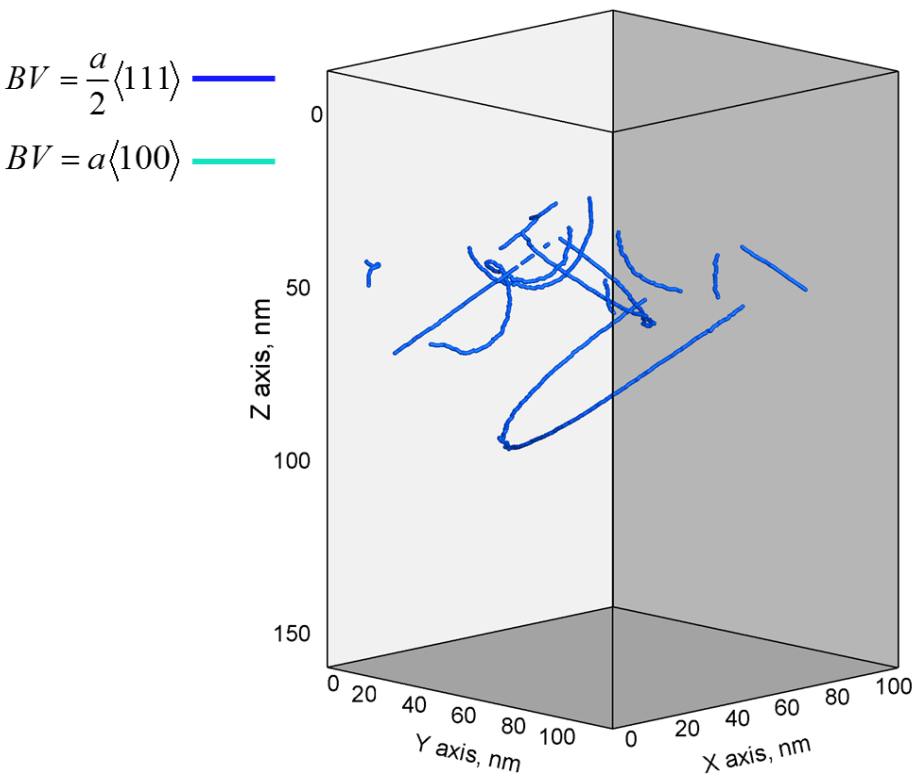

$350 \mathrm{ps}$

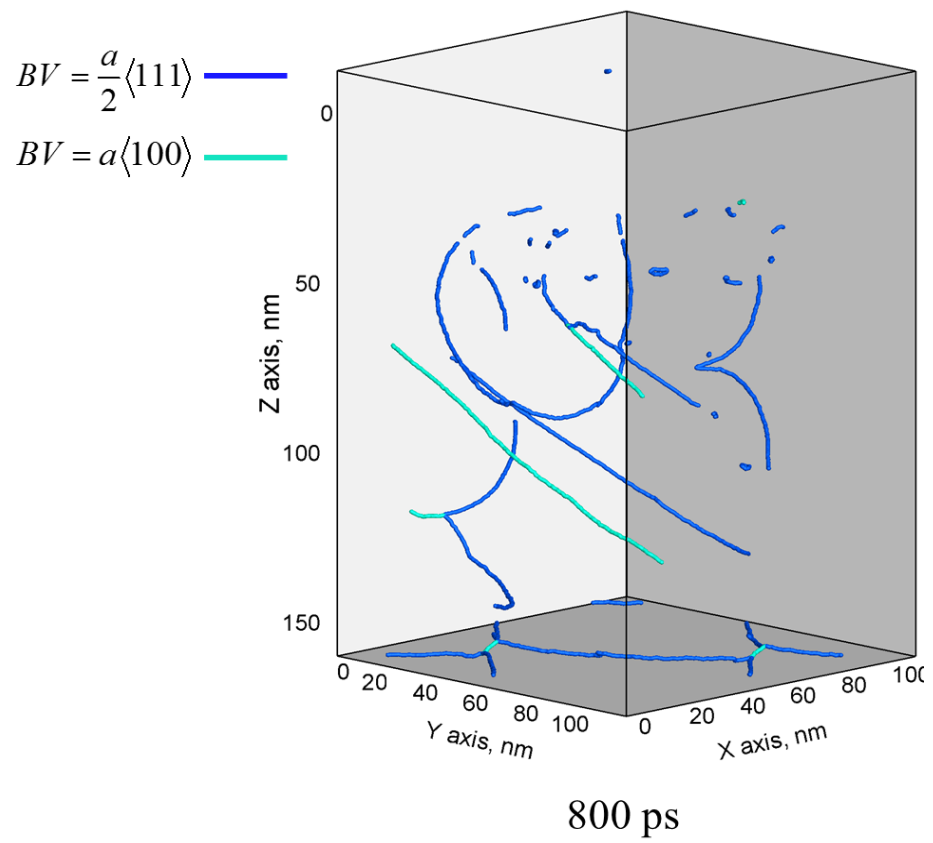

Fig. 3.10. Snapshots of defect structures generated in the surface region of a bulk (001) Cr target irradiated by a $50 \mathrm{fs}$ laser pulse at an absorbed laser fluence of $1100 \mathrm{~J} / \mathrm{m}^{2}$, slightly above the threshold for the transition from melting to the spallation regime. The snapshots are quenched to $0 \mathrm{~K}$ for $2 \mathrm{ps}$ and then the "Dislocation Extraction Algorithm", DXA, is used to define the dislocation lines and to determine their Burgers vectors. The snapshots are taken at different simulation times to illustrate the evolution of the dislocations in the system. Each point in the snapshots represents a dislocation segment and each segment is colored according to its Burgers vector, with blue and light green colors corresponding to Burgers vectors $\frac{a}{2}\langle 111\rangle$ and $a\langle 100\rangle$ Burgers vector. 
Another type of perfect dislocation that is observed in the target is the one with Burgers vectors $a\langle 100\rangle$. The dislocations of this type result from the interaction of dislocations with Burgers vectors $\frac{a}{2}\langle 111\rangle$, where the reaction can be written as $\frac{a}{2}[111]+\frac{a}{2}[1 \overline{1} \overline{1}] \rightarrow a[100]$. The dislocations of this type are unlikely to contribute in the plastic deformation but they may play a role in the nucleation of micro-cracks in the crystal [195]. At the end of the simulation, $800 \mathrm{ps,}$ the maximum lengths and density of the dislocations of type $\frac{a}{2}\langle 111\rangle$ reach $\sim 950 \mathrm{~nm}$ and $7.3 \times 10^{-4}$ $\mathrm{nm}^{-2}$ respectively, while the total length and the maximum density of the dislocations of type $a\langle 100\rangle$ approaches $172 \mathrm{~nm}$ and $1.32 \times 10^{-4} \mathrm{~nm}^{-2}$, respectively. The analysis of the crystal defects indicates the absence of dislocations in the eight spikes on the surface of the target. The spikes with diameters ranged between 10 and $30 \mathrm{~nm}$ are similar to nanowires or nanowhiskers, therefore, and the, free surfaces of the spikes can strongly attract the dislocations and leave the spikes free of crystal defects.

\subsubsection{Generation of perfect dislocations in (110) and (111) Cr targets}

In order to investigate the dependence of the dislocation emission on the crystallographic orientation of the irradiated target, two large-scale TTM-MD simulations of (110) and (111) $\mathrm{Cr}$ targets irradiated with a 50 fs laser pulse at an absorbed laser fluence of $800 \mathrm{~J} / \mathrm{m}^{2}$ are carried out and the results are compared with the ones discussed above for the (001) Cr target. The temporal and spatial evolution of the lattice temperature and pressure shown in Fig. 3.5 is mostly similar in the three targets. The main difference, however, is the distinct presence of quasi-static compressive stresses in the pressure contour plot of (001) target until the end of the simulation, Fig. 3.5(a). In contrast, this residual compressive stresses are partially relaxed in the (110) and (111) targets due to the emission and propagation of perfect dislocations in the regime of melting and solidification.

To support our hypothesis of the correlation between the dislocations emission and the relaxation of the residual compressive stresses, several snapshots of the atomic configurations obtained from large-scale TTM-MD simulations for (110) and (111) targets are quenched to $0 \mathrm{~K}$ for 2 ps by using the velocity damping technique and the results are presented in Figs. 3.11 and 3.12 for (110) and (111) targets, respectively. The atoms in these snapshots are colored 
according to their potential energies and the same coloring scale that is used in Figs. 3.7 and 3.9 is used in these snapshots. All the atoms that belong to the original bcc crystal structure and have potential energies below $-3.99 \mathrm{eV}$ are blanked and only the atoms that belong to the free surface, liquid phase or crystal defects are shown. The crystal defects in these snapshots are marked as " $A$ " for the point defects, vacancies and self-interstitials, and for the " $B$ " as perfect dislocations.

The snapshots illustrate that the rapid increase of the lattice temperature leads to the formation of liquid regions inside the overheated lattice and allows for the homogeneous melting to take place in the targets, as can be seen from the existence of liquid islands ahead of the melting front at a time of $10 \mathrm{ps}$ in both targets. Meanwhile, the overheating of the lattice below the melting front results in a thermally activated generation of several vacancy-interstitial pairs which is termed "Frenkel pair". At 20 ps from the laser pulse, the emission of dislocations from the melting fronts and their propagation deeper into the targets is observed along with the expansion of the depth of the melted region. The density of the dislocations increases with continued emission of new dislocations and the dislocation multiplication. The snapshots reveal the fast propagation of the dislocations in (110) target down to the bottom of the computational system, where they are repelled by the layer of atoms that belongs to the non-reflecting boundary. A slower motion of the dislocations is observed in the (111) target, where the dislocations reach the depth of $115 \mathrm{~nm}$ below the surface by the end of the simulation.

In addition to the perfect dislocations, the generation of high density of vacancies is also observed during the solidification process, and the formation of vacancies deeper inside the bulk of the targets due to the non-conservative propagation of the dislocations, as can be seen from Figs. 3.11 and 3.12 . 

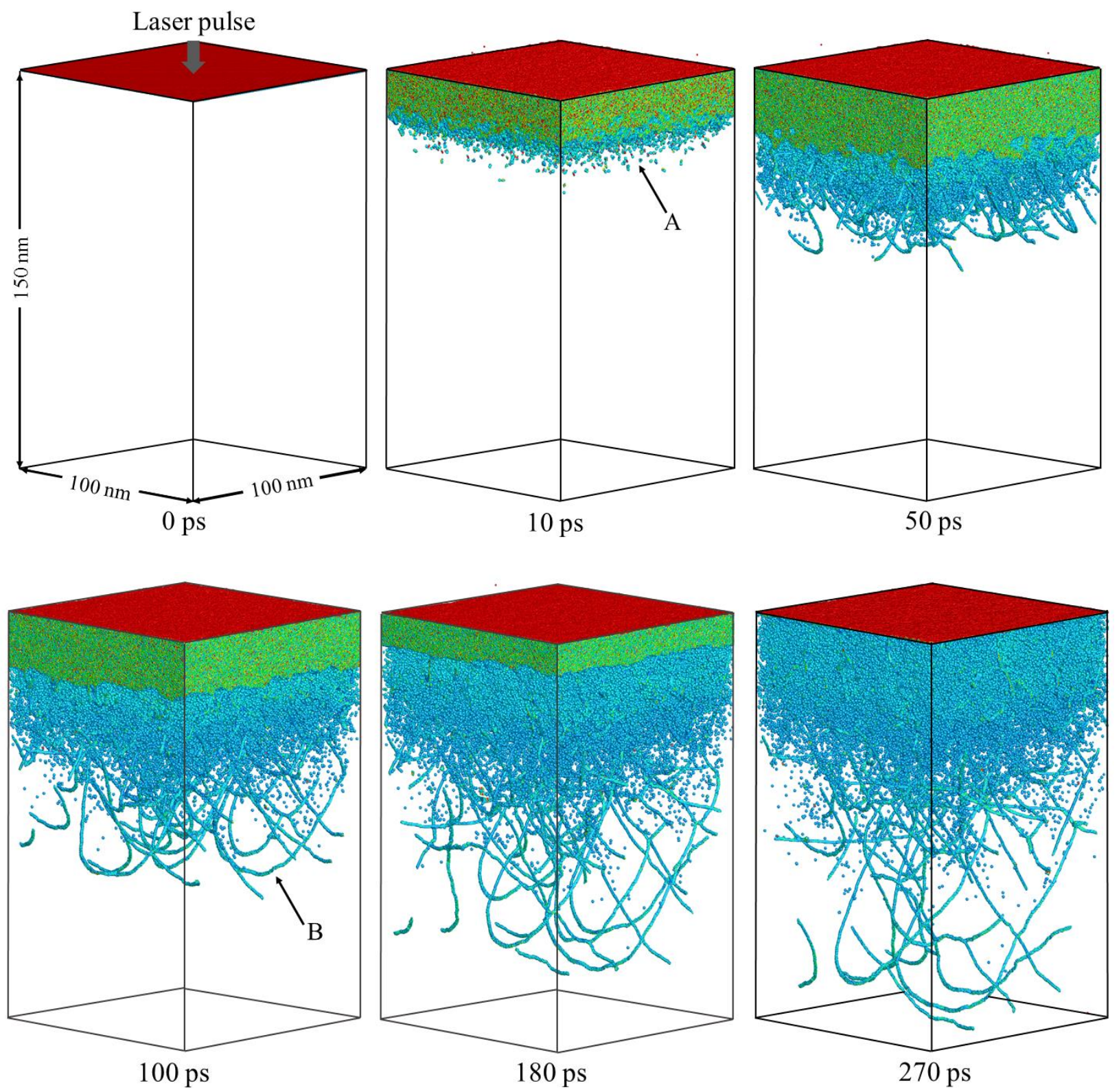

Fig. 3.11. Snapshots of the atomic configurations obtained in large-scale TTM-MD simulation of a bulk (110) Cr target irradiated with a $50 \mathrm{fs}$ laser pulse at an absorbed laser fluence of $800 \mathrm{~J} / \mathrm{m}^{2}$, which is $66 \%$ above the threshold for surface melting. The snapshots are quenched to $0 \mathrm{~K}$ for $2 \mathrm{ps}$ in order to reduce thermal noise in atomic positions and energies. Atoms are colored according to their potential energies and all the atoms that have energies below $-3.99 \mathrm{eV}$ and belong to the original bcc crystal structure are blanked to display the crystal defects. The red atoms in the top layer of the snapshots belong to the free surface of the target, the green atoms represent the melting region and the blue atoms represent the crystal defects. The crystal defects marked by $A$ in a snapshot shown for 10 ps correspond to the point defects, while the one marked by $B$ in a snapshot shown for 100 ps are the perfect dislocations. 

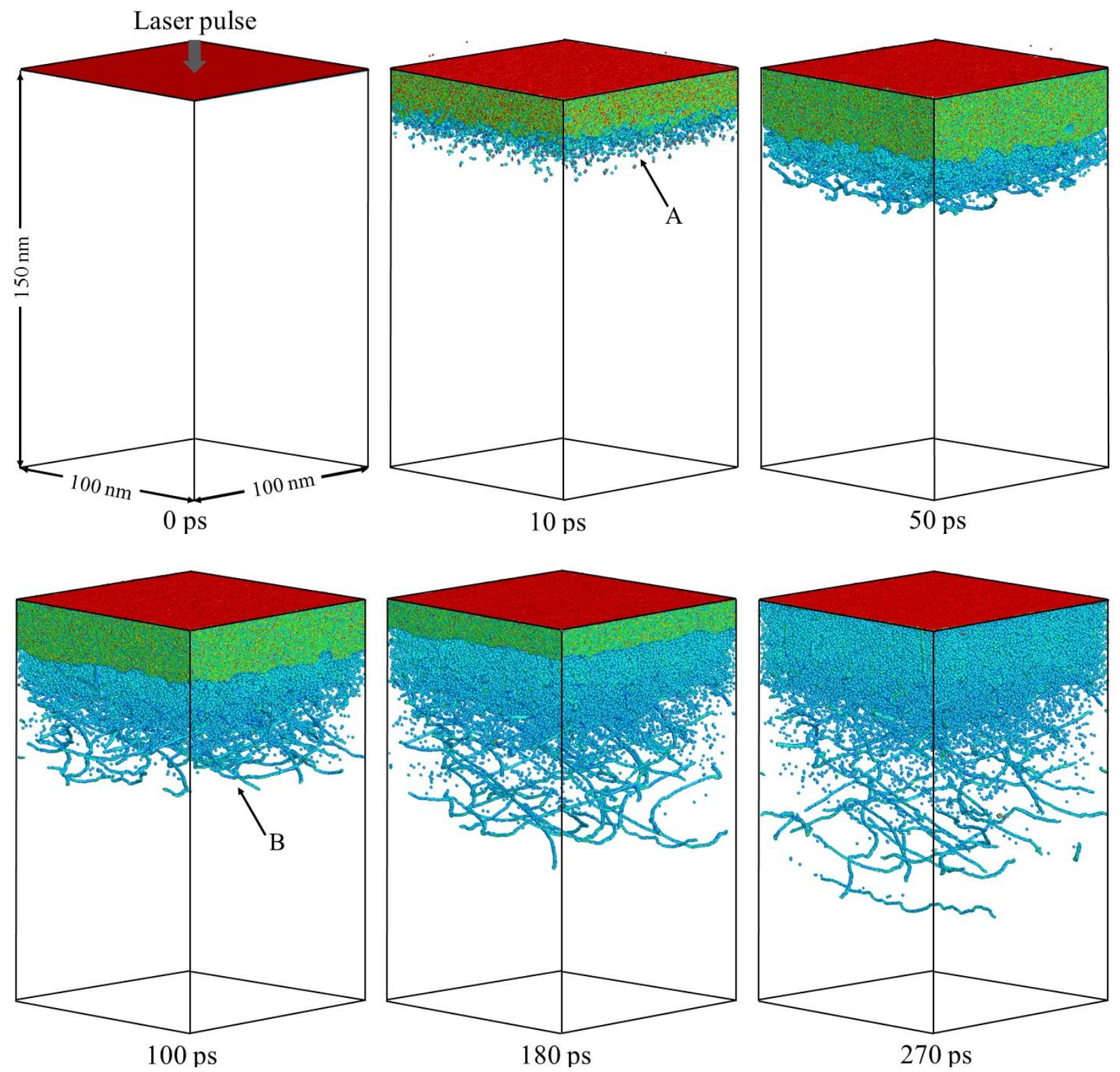

Fig. 3.12. Snapshots of the atomic configurations obtained in large-scale TTM-MD simulation of a bulk (111) Cr target irradiated with a $50 \mathrm{fs}$ laser pulse at an absorbed laser fluence of $800 \mathrm{~J} / \mathrm{m}^{2}$, which is $66 \%$ above the threshold for surface melting. The snapshots are quenched to $0 \mathrm{~K}$ for $2 \mathrm{ps}$ in order to reduce thermal noise in atomic positions and energies. Atoms are colored according to their potential energies and all the atoms that have energies below $-3.99 \mathrm{eV}$ and belong to the original bcc crystal structure are blanked to display the crystal defects. The red atoms in the top layer of the snapshots belong to the free surface of the target, the green atoms represent the melting region and the blue atoms represent the crystal defects. The crystal defects marked by $A$ in a snapshot shown for 10 ps correspond to the point defects, while the one marked by $B$ in a snapshot shown for 100 ps are the perfect dislocations. 
In order to provide a better understanding on the dislocation emission in both systems, the resolved shear stress is calculated for the 48 slip systems for atomic configurations generated in the two simulations and the results are discussed in more detailed in section 3.5.3. In general, in both targets, the increase of the resolved shear stress directly below the melting front to more than $3 \mathrm{GPa}$ at the early time of the simulations along with the high compressive stresses that ranged between 2.5 and $3 \mathrm{GPa}$, can be the key to the understanding of the appearance of the dislocations in the targets. Simultaneously, the absence of the generation of the unstable stacking faults in response to the uniaxial expansion of the two targets can be clarified by calculating the Schmid factor for the generation of unstable stacking faults along the $\{011\}$ planes in the three targets (001), (110), and (111). These results of the calculation indicate that the Schmid factor reaches its maximum value of 0.5 in four out of six $\{011\}$ planes for (001) target and reduces to 0.25 and zero for (110) and (111) targets, respectively. These results indicate that the low value of the Schmid factor prevents the generation of the stacking faults in the two targets.

The analysis of the defects structures for the two systems is also performed with the Dislocation Extraction Algorithm, DXA, and the snapshots representing the evolution of the dislocations in the two systems are shown in Figs. 3.13 and 3.14 for the (110) and (111) targets, respectively. Similar to the analysis of the results of crystal defects for the (001) target in the photomechanical spallation regime (Fig. 3.10), each point in these snapshots represents a dislocation segment and each segment is colored according to its Burgers vector, with the blue and light green colors corresponding to dislocation segment with $\frac{a}{2}\langle 111\rangle$ and $a\langle 100\rangle$ Burgers vectors. The snapshots illustrate the emission of perfect dislocations with Burgers vectors $\frac{a}{2}\langle 111\rangle$ from the melting fronts and their subsequent propagation deeper into the targets. The solidification process also introduces new dislocations into the targets, with a pronounced anisotropic orientation of the dislocation lines along the direction of the solidification front propagation. The two distinct mechanisms of the dislocation generation are discussed below. 

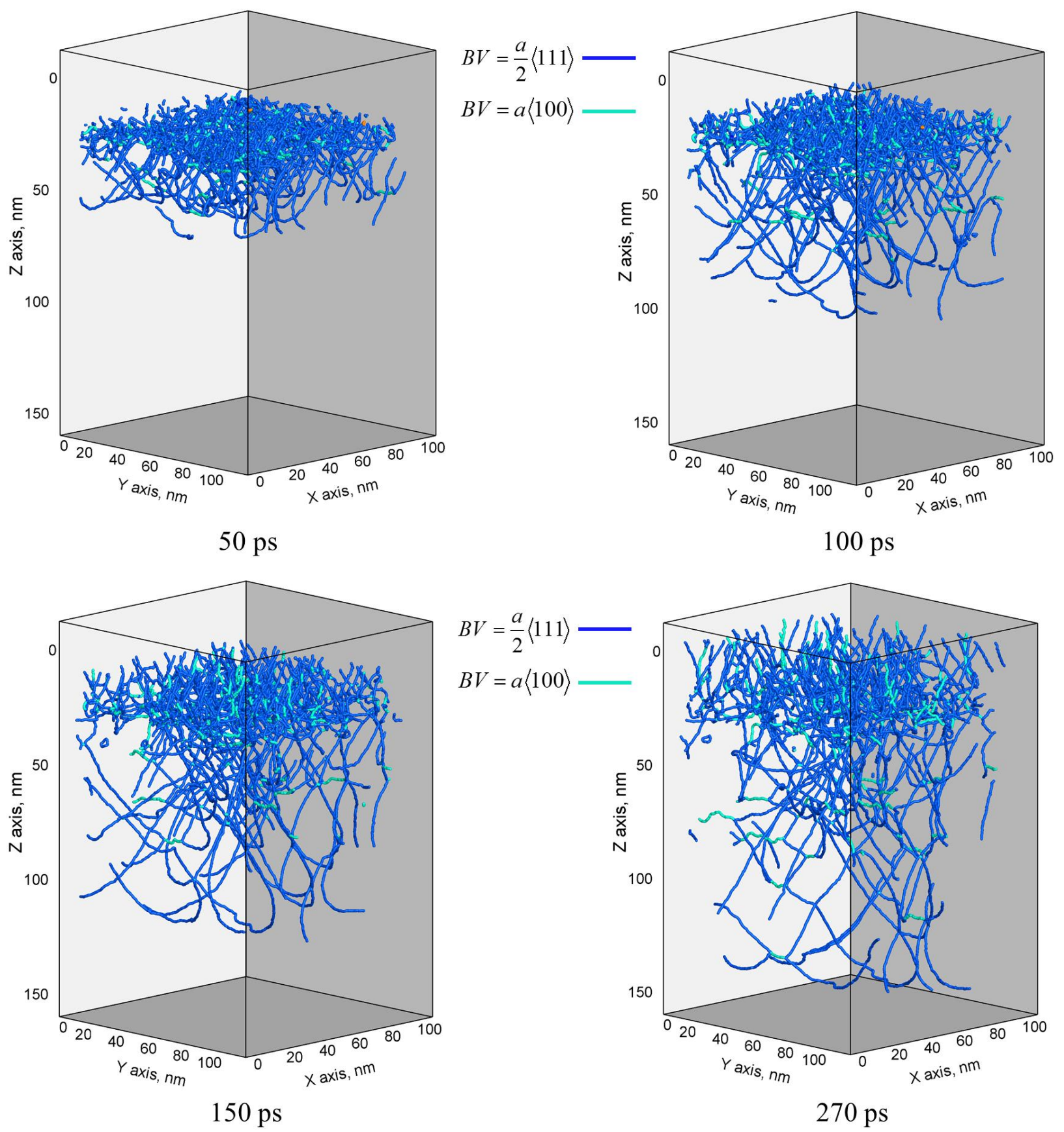

Fig. 3.13. Snapshots of defect structures generated in the surface region of a bulk (110) Cr target irradiated by a $50 \mathrm{fs}$ laser pulse at an absorbed laser fluence of $800 \mathrm{~J} / \mathrm{m}^{2}$, which is $66 \%$ above the threshold for the transition to the melting regime. The snapshots are quenched to $0 \mathrm{~K}$ for $2 \mathrm{ps}$ and then analyzed by using the "Dislocation Extraction Algorithm", DXA, in order to define the dislocation lines and to determine their Burgers vectors. The snapshots are taken at different simulation times to illustrate the evolution of the dislocations in the system. Each point in the snapshots represents a dislocation segment and each segment colored according to its Burgers vector: the blue segments have Burgers vectors of $\frac{a}{2}\langle 111\rangle$ and, the light green ones have Burgers vectors of $a\langle 100\rangle$. 


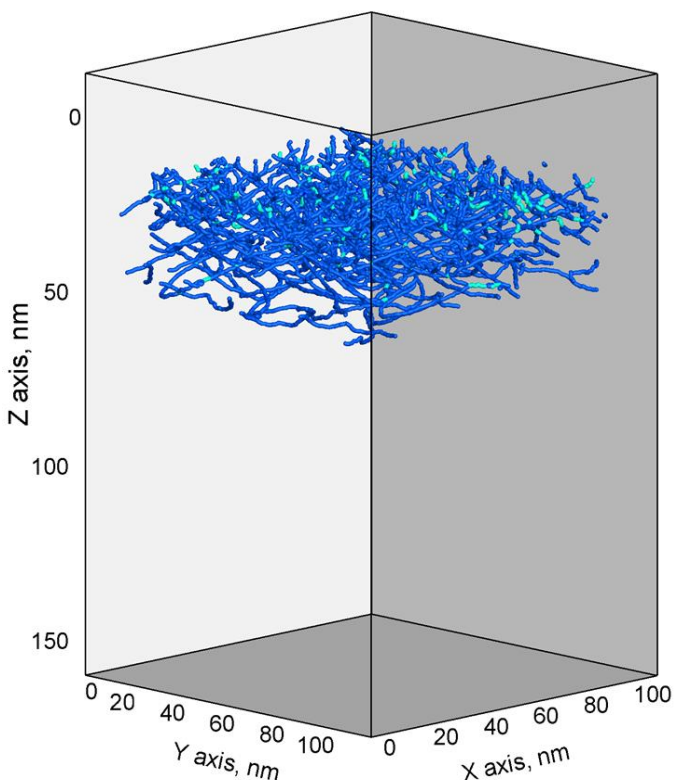

$70 \mathrm{ps}$

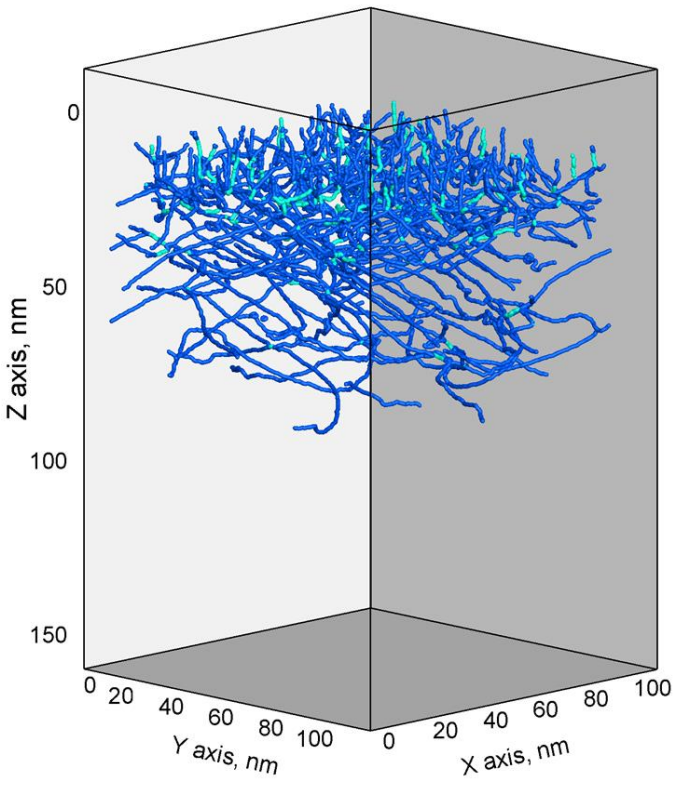

$150 \mathrm{ps}$

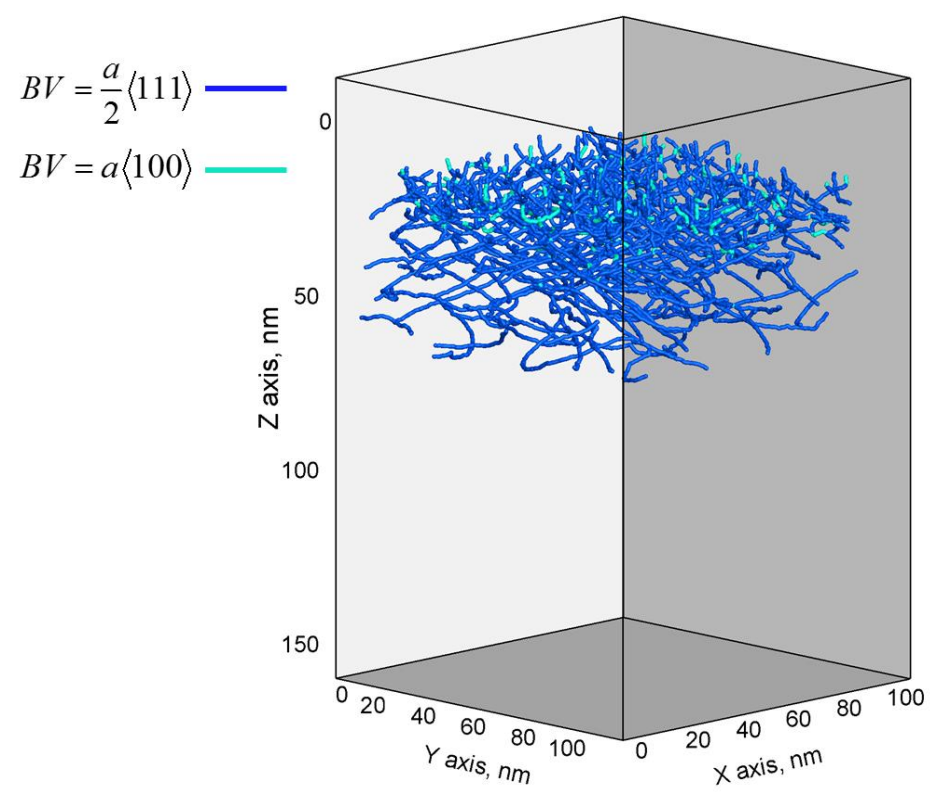

$100 \mathrm{ps}$

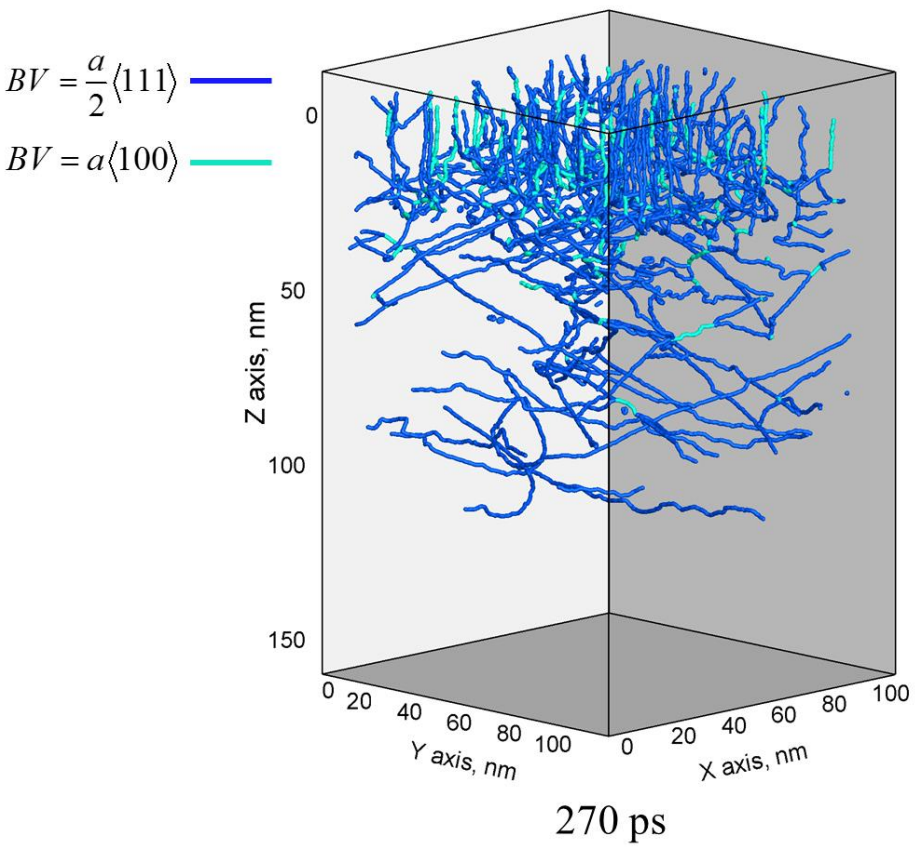

Fig. 3.14. Snapshots of defect structures generated in the surface region of a bulk (111) Cr target irradiated by a $50 \mathrm{fs}$ laser pulse at an absorbed laser fluence of $800 \mathrm{~J} / \mathrm{m}^{2}$, which is $66 \%$ above the threshold for the transition to the melting regime. The snapshots are quenched to $0 \mathrm{~K}$ for $2 \mathrm{ps}$ and then analyzed by using the "Dislocation Extraction Algorithm", DXA, in order to define the dislocation lines and to determine their Burgers vectors. The snapshots are taken at different simulation times to illustrate the evolution of the dislocations in the system. Each point in the snapshots represents a dislocation segment and each segment colored according to its Burgers vector: the blue segments have Burgers vectors of $\frac{a}{2}\langle 111\rangle$ and, the light green ones have Burgers vectors of $a\langle 100\rangle$. 
The first mechanism of the dislocation generation is related to the emission and propagation of the dislocations due to the action of the resolved shear stresses on the corresponding slip systems activated in the target during the time of the dynamic relaxation of the laser-induced stresses. As discussed in detail below, in section 3.5.3, the resolved shear stress in the two targets reaches its maximum levels of $3.5-3.9 \mathrm{GPa}$ at a depth of $\sim 30 \mathrm{~nm}$ below the surface and the time of 20 ps after the laser pulse. The high levels of the shear stress along with the high temperature into the targets of the surface region are responsible for the prompt emission and rapid propagation of the dislocations. The second mechanism is correlated to the natural requirement for the dislocations to end at the surface of the crystal (or at the liquid-crystal interface), resulting in the continuing connection of the dislocations to the solidification front during the resolidification process. As the solidification front propagates toward the surface of the target, it pulls the dislocations along, creating an effective vertically-aligned "forest" of the dislocations in the resolidified part of the target. The dislocation segments tend to be inclined with an angle in the case of (110) target as compared to the straight growth of the dislocations toward the surface for (111) target. The reason of this behavior of the dislocations in (110) target may be related to the tendency of the dislocations to grow along the closed packed direction, while in Cr with (111) surface, the crystallographic orientation of the irradiated target is set to be along $\langle 111\rangle$ and result in the straight grow of the dislocation toward the surface.

Similar to the analysis of the dislocations generated in the (001) Cr target irradiated in the photomechanical spallation regime (Fig. 3.10), the results of the two simulations performed for (011) and (111) Cr targets also reveal the formation of dislocations with Burgers vectors of $a\langle 100\rangle$. Typically, in a crystal with high density of dislocations, the dislocations tend to interact with each other in order to reduce the long-range elastic fields. The reduction of the elastic strain energy per unit length, where the energy of the dislocation is directly proportional to the square of the magnitude of its Burgers vector, can be tested by using Frank's rule in order to determine if the reaction is energetically favorable. Accordingly, the reaction between two dislocations with Burgers vectors of $\frac{a}{2}\langle 111\rangle, \frac{a}{2}[111]+\frac{a}{2}[1 \overline{1} \overline{1}] \rightarrow a[100]$, would result in the reduction of the elastic strain energy per unit length and would be energetically favorable, since the condition of $\left.b_{1}^{2}+b_{2}^{2}\right\rangle b_{3}^{2}$ is satisfied: $\left.\frac{3 a^{2}}{4}+\frac{3 a^{2}}{4}\right\rangle a^{2}$. Fig. 3.15(a) and (b) illustrate the interaction between two 
dislocations with Burgers vectors $\frac{a}{2}\langle 111\rangle$ that results in the formation of a dislocation segment with a Burgers vector of $a\langle 100\rangle$. Therefore, we can infer that the formation of dislocations with Burgers vectors $a\langle 100\rangle$ is due to the reactions between dislocations with Burgers vectors $\frac{a}{2}\langle 111\rangle$. Several dislocation loops with Burgers vectors $\frac{a}{2}\langle 111\rangle$ are also observed in the two simulations at locations with a high density of vacancies, Figs. 3.15(c), 3.13, and 3.14. With the existence of a high density of vacancies along with the rapid undercooling process, the dislocation loops are believed to form initially with Burgers vectors $\frac{a}{2}\langle 110\rangle$ on $\{110\}$ planes and then shear to become perfect dislocations following the reaction: $\frac{a}{2}\langle 110\rangle+\frac{a}{2}\langle 001\rangle \rightarrow \frac{a}{2}\langle 111\rangle$.

At a time of $270 \mathrm{ps}$ and after the complete solidification of both systems, high density of dislocations is generated in the surface regions of the (110) and (111) Cr targets. In the (110) $\mathrm{Cr}$ target, the densities of dislocations with Burgers vectors $\frac{a}{2}\langle 111\rangle$ and $a\langle 100\rangle$ are $0.72 \times 10^{-2} \mathrm{~nm}^{-2}$ and $\sim 0.1 \times 10^{-2} \mathrm{~nm}^{-2}$, respectively. Similar dislocation densities are calculated for the (111) $\mathrm{Cr}$ target: $0.69 \times 10^{-2} \mathrm{~nm}^{-2}$ for dislocations with Burgers vector $\frac{a}{2}\langle 111\rangle$ and $0.1 \times 10^{-2} \mathrm{~nm}^{-2}$ for dislocations with Burgers vectors $a\langle 100\rangle$. 


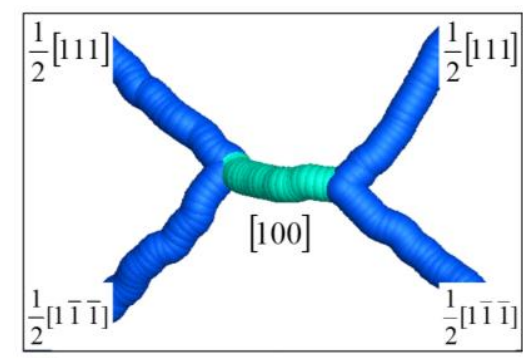

(a)

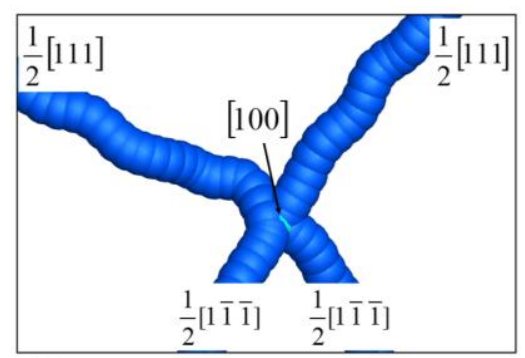

(b)

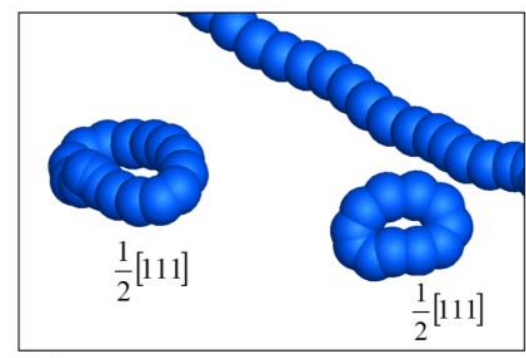

(c)

Fig. 3.15. Enlarged views of elements of dislocation structures generated in the surface region of bulk (110) and (111) Cr targets irradiated by a 50 fs laser pulse at an absorbed laser fluence of $800 \mathrm{~J} / \mathrm{m}^{2}$, which is $66 \%$ above the threshold for the transition to the melting regime. Each point in the snapshots represents a dislocation segment and each segment is colored according to its Burgers vector, with blue and light green colors corresponding to dislocations with Burgers vectors of $\frac{a}{2}\langle 111\rangle$ and $a\langle 100\rangle$, respectively. The configurations shown in (a) and (b) represent interaction between two dislocations with Burgers vector $\frac{a}{2}\langle 111\rangle$ that results in the generation of a dislocation segment with Burgers vector $a\langle 100\rangle$. The configuration in (c) represents a dislocation loops with Burgers vector $\frac{a}{2}\langle 111\rangle$.

\subsubsection{Analysis of the resolved shear stress in (001), (110), (111) Cr targets}

The contrast between the appearance of dislocations in the (110) and (111) Cr targets and their absence in (001) $\mathrm{Cr}$ target in the melting regime, as well as the appearance of dislocations in (001) Cr target in the spallation regime, call for a better understanding of the conditions that lead to the emission of the dislocations in the irradiated targets and the dependence of these conditions on the crystallographic orientation of the target surface. Accordingly, the calculation of the resolved shear stresses in the 48 slip systems is performed for the four large-scale simulations discussed in the previous sections. The calculation is done using the general form of the Schmid's law given by the following equation $\tau_{n d}=\sum_{k=1}^{3} \sum_{m=1}^{3} l_{n m} l_{d k} \sigma_{m k}$, where $n$ and $d$ refer to the normal to the slip plane and the direction of the slip, respectively, $l_{n m}$ is the cosine of the angle between the uniaxial force and the normal to the slip plane, and $l_{d k}$ is the cosine of the angle between the uniaxial force and the slip direction. 
Table 3.1 provides the results of the maximum resolved shear stress for the 48 slip systems in (001), (110), and (111) Cr targets. In this Table, all the values with maximum resolved shear stress that are above $3 \mathrm{GPa}$ are marked in order to compare the results for the three systems. The value of $3 \mathrm{GPa}$ is higher than the yield strength of the $\mathrm{Cr}$, under conditions of slow quasi-static loading, $362 \mathrm{MPa}$, and lower than the theoretical resolved shear stress for this metal, $3.8 \mathrm{GPa}$. The results illustrate that the maximum value of the resolved shear stress is 2.93 , 3.68, and 4.00 GPa for (001), (110) and (111) targets, respectively, which is observed in several slip systems of $\{112\}<111>$ type. Although, the maximum value of the resolved shear stress in the (001) target is only moderately lower than the ones in the (110) and (111) targets, the snapshots of atomic configurations along with the analysis of the pressure contour plots show only the transit generation of stacking faults in this simulation. To more shed light on the difference between the three systems, the temporal and spatial evolution of the resolved shear stress is plotted for the slip planes in Fig. 3.16. The pressure plots with the maximum tensile and compressive values denoted are also provided in the same figure. 


\begin{tabular}{|c|c|c|c|c|c|c|c|c|c|}
\hline \multicolumn{2}{|c|}{ Slip systems in $\mathrm{Cr}$} & \multirow{2}{*}{$\begin{array}{c}\text { Max. RSS. } \\
(001), \mathrm{GPa} \\
0.02\end{array}$} & \multirow{2}{*}{$\begin{array}{c}\text { Max. RSS. } \\
\text { (110), GPa } \\
-0.209\end{array}$} & \multirow{2}{*}{$\begin{array}{c}\text { Max. RSS. } \\
\text { (111), GPa } \\
\\
-3.190\end{array}$} & \multicolumn{2}{|c|}{ Slip systems in $\mathrm{Cr}$} & \multirow{2}{*}{$\begin{array}{c}\text { Max. RSS. } \\
(001), \mathrm{GPa} \\
2.880\end{array}$} & \multirow{2}{*}{$\begin{array}{c}\text { Max. RSS. } \\
(110), \mathrm{GPa} \\
2.433\end{array}$} & \multirow{2}{*}{$\begin{array}{c}\text { Max. RSS. } \\
\text { (111), GPa } \\
\\
-3.607\end{array}$} \\
\hline 1. & $(110)[\overline{1} 11]$ & & & & 1. & $(123)[11 \overline{1}]$ & & & \\
\hline 2. & $(110)[1 \overline{1} 1]$ & 0.02 & -0.218 & -3.186 & 2. & $(\overline{1} 23)[1 \overline{1} 1]$ & -2.883 & 3.931 & -2.418 \\
\hline 3. & $(1 \overline{1} 0)[111]$ & 0.014 & -0.026 & -0.036 & 3. & $(1 \overline{2} 3)[\overline{1} 11]$ & -2.880 & 3.934 & -1.212 \\
\hline 4. & $(1 \overline{1} 0)[11 \overline{1}]$ & 0.015 & 0.038 & -0.175 & 4. & $(12 \overline{3})[111]$ & 2.884 & 2.430 & -0.032 \\
\hline 5. & $(101)[11 \overline{1}]$ & 2.543 & 2.146 & -3.180 & 5. & (132)[1 $1 \overline{1} 1]$ & -1.925 & 2.617 & -3.618 \\
\hline 6. & $(101)[\overline{1} 11]$ & -2.542 & 3.472 & -3.194 & 6. & $(\overline{1} 32)[11 \overline{1}]$ & 1.919 & 1.621 & -2.407 \\
\hline 7. & $(10 \overline{1})[111]$ & 2.542 & 2.140 & -0.044 & 7. & $(1 \overline{3} 2)[111]$ & -1.924 & -1.623 & 0.029 \\
\hline 8. & $(10 \overline{1})[1 \overline{1} 1]$ & 2.543 & -3.470 & -0.185 & 8. & $(13 \overline{2})[\overline{1} 11]$ & 1.919 & -2.620 & -1.204 \\
\hline 9. & $(011)[11 \overline{1}]$ & 2.539 & 2.145 & -3.182 & 9. & $(213)[11 \overline{1}]$ & 2.881 & 2.433 & -3.606 \\
\hline 10 & $(011)\left[\begin{array}{lll}1 & 1 & 1\end{array}\right]$ & -2.544 & 3.465 & -3.194 & 10. & $(\overline{2} 13)[1 \overline{1} 1]$ & -2.883 & 3.933 & -1.214 \\
\hline 11. & $(01 \overline{1})[111]$ & 2.544 & 2.145 & -0.027 & 11. & $(2 \overline{1} 3)[\overline{1} 11]$ & -2.881 & 3.935 & -2.417 \\
\hline 12. & $(01 \overline{1})[\overline{1} 11]$ & 2.539 & -3.468 & -0.148 & 12. & $(21 \overline{3})[111]$ & 2.883 & 2.429 & -0.0412 \\
\hline 13. & $(112)[11 \overline{1}]$ & 2.933 & 2.478 & -3.672 & 13. & $(231)[1 \overline{1} 1]$ & -0.965 & 1.306 & -3.615 \\
\hline 14. & $(\overline{1} 12)[1 \overline{1} 1]$ & -2.936 & 4.000 & -1.849 & 14. & $(\overline{2} 31)[11 \overline{1}]$ & 0.959 & 0.8111 & -1.207 \\
\hline 15. & $(1 \overline{1} 2)[\overline{1} 11]$ & -2.933 & 4.000 & -1.848 & 15. & $(2 \overline{3} 1)[111]$ & -0.965 & -0.815 & 0.0313 \\
\hline 16. & $(11 \overline{2})[111]$ & 2.936 & 2.474 & -0.0370 & 16. & $(23 \overline{1})[\overline{1} 11]$ & 0.963 & -1.308 & -2.410 \\
\hline 17. & $(121)[1 \overline{1} 1]$ & -1.471 & 1.997 & -3.683 & 17. & $(312)[\overline{1} 11]$ & -1.923 & 2.626 & -3.620 \\
\hline 18. & $(\overline{1} 21)[11 \overline{1}]$ & 1.466 & 1.238 & -1.839 & 18. & $(\overline{3} 12)[111]$ & -1.922 & -1.616 & 0.044 \\
\hline 19. & $(1 \overline{2} 1)[111]$ & -1.471 & -1.241 & 0.028 & 19. & $(3 \overline{1} 2)[11 \overline{1}]$ & 1.924 & 1.623 & -2.403 \\
\hline 20. & $(12 \overline{1})[\overline{1} 11]$ & 1.467 & -2.000 & -1.840 & 20. & $(31 \overline{2})[1 \overline{1} 1]$ & 1.923 & -2.625 & -1.199 \\
\hline 21. & $(211)\left[\begin{array}{ll}1 & 11\end{array}\right]$ & -1.470 & 2.007 & -3.685 & 21. & $(321)[\overline{1} 11]$ & -0.964 & 1.315 & -3.618 \\
\hline 22. & $(\overline{2} 11)[111]$ & -1.467 & -1.233 & 0.419 & 22. & $(\overline{3} 21)[111]$ & -0.960 & -0.806 & 0.038 \\
\hline 23. & $(2 \overline{1} 1)[11 \overline{1}]$ & 1.472 & 1.240 & -1.836 & 23. & $(3 \overline{2} 1)[11 \overline{1}]$ & 0.967 & 0.812 & -1.202 \\
\hline 24. & $(21 \overline{1})[1 \overline{1} 1]$ & 1.471 & -2.006 & -1.835 & 24. & $(32 \overline{1})[1 \overline{1} 1]$ & 0.965 & -1.316 & -2.405 \\
\hline
\end{tabular}

Table. 3.1. The maximum values of the resolved shear stress calculated for the 48 slip systems in the (001), (110), and (111) Cr targets. The three targets are irradiated with a 50 fs laser pulse at an absorbed laser fluence of $800 \mathrm{~J} / \mathrm{m}^{2}$, which is $66 \%$ above the threshold for melting and solidification. All the values of the maximum resolved shear stress that are above $3 \mathrm{GPa}$ are marked with gray color. The $3 \mathrm{GPa}$ is lower than the theoretical resolved shear stress for $\mathrm{Cr}, 3.8 \mathrm{GPa}$. 
Starting with the (001) target and paying more attention to the slip system (112)[11 $\overline{1}]$, where the maximum resolved shear stress of $2.93 \mathrm{GPa}$ is observed, Table 3.1, let us consider the spatial and time evolution of the resolved shear stress and pressure in this target. The most notable observation from the plot of the resolved shear stress shown in Fig. 3.16(a) is that the values of the shear stress remain rather low in the vicinity of the melting front and do not exceed $\sim 130 \mathrm{MPa}$ during the first 20 ps from the laser pulse, which is even below the yield strength of Cr. This observation can be attributed to the presence of an alternative relaxation of the shear stresses through internal slip along $\{011\}$ planes leading to the transient appearance of the large number of stacking faults (see discussion in section 3.5.1). While retraction of the stacking faults leads to an increase in the shear stress below the liquid-crystal interface up to $\sim 1.2 \mathrm{GPa}$ in twelve slip systems, it is not high enough to cause the emission of the dislocations. The inspection of the location of the maximum resolved shear stress in this system indicates that the maximum value, $\sim 2.93 \mathrm{GPa}$, is reached at a depth of $120 \mathrm{~nm}$ below the surface at about $40 \mathrm{ps}$ after the laser pulse. The temperature at this depth is equal to $\sim 0.3 T_{m}$, as can be seen from Fig. 3.5(a) and the increase of the resolved shear stress to $2.93 \mathrm{GPa}$ is not sufficient for the homogeneous nucleation of dislocation loops inside the uniaxially expanded crystal.

A very different picture of the evolution of the pressure and the shear stress resolved for the slip system( $(\overline{1} 12)[1 \overline{1} 1]$ is observed in Fig. 3.16(b) for the (110) target. The maximum value of the resolved shear stress in this target is ranging between 3.5 and $4 \mathrm{GPa}$, with the shear stress approaching this value in several slip systems at about 20 ps from the laser pulse at a depth of about $30 \mathrm{~nm}$ below the surface. The generation of strong shear stresses in the immediate vicinity of the melting front, along with the high lattice temperature approaching and even exceeding the melting temperature of $\mathrm{Cr}$ create the conditions for the massive dislocation emission from the melting front. Meanwhile, the propagation of the dislocations deeper into the target is assisted by the pressure oscillations, the elevated lattice temperature, and the quasi-static uniaxial lattice expansion that maintains relatively high level of resolved shear stress, $\sim 1 \mathrm{GPa}$, in the region that is not yet reached by the dislocations. The propagation of the dislocations deeper into the target reduces the shear stresses and result in the relaxation of the residual compressive pressure from 1.7 GPa to less than $1 \mathrm{GPa}$ by the end of the simulation. 


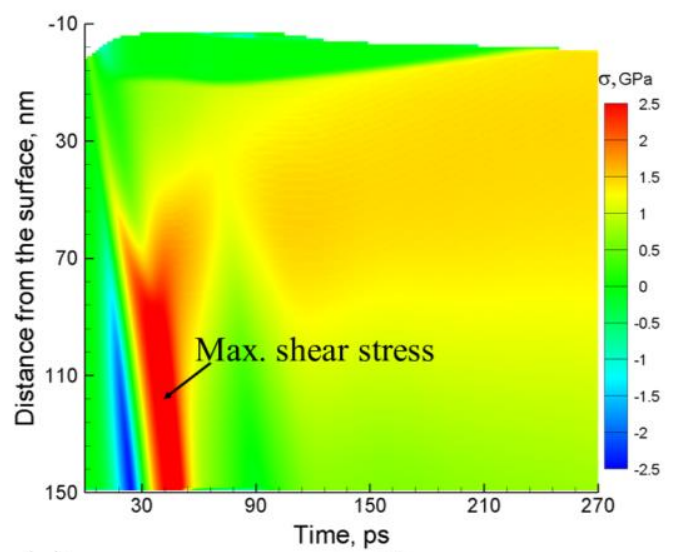

(a)

(112) [11 $\overline{1}]$

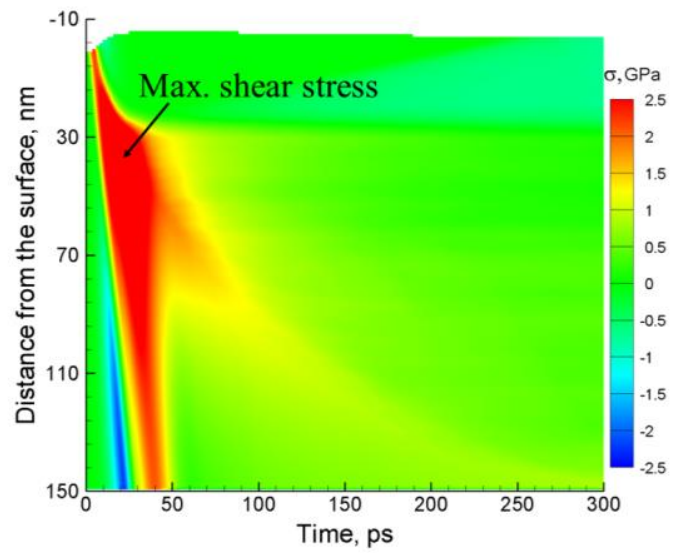

(b)

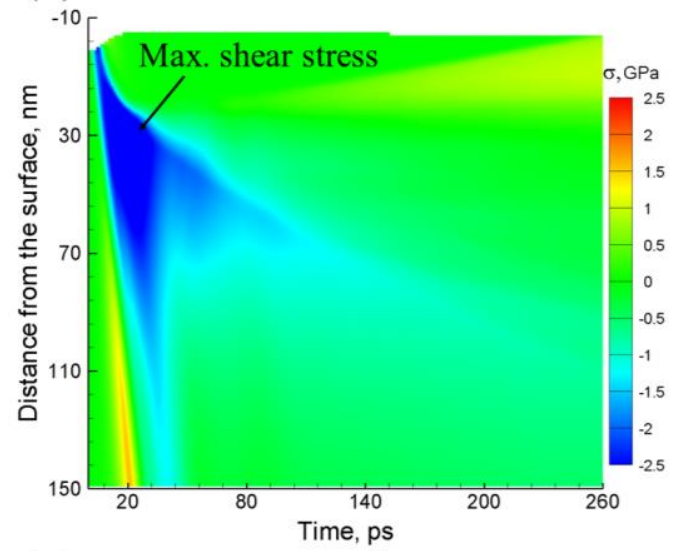

(c)
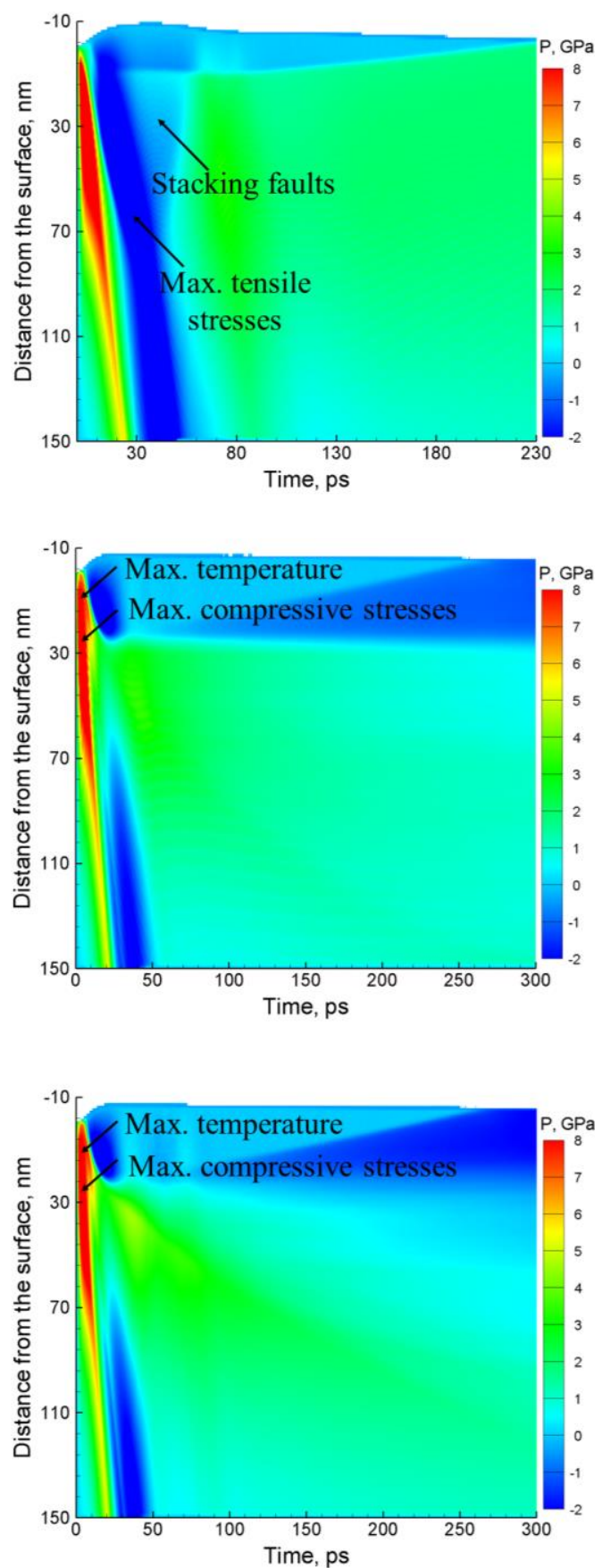

Fig. 3.16. Contour plots of the spatial and temporal evolution of the resolved shear stress (left) and pressure (right) obtained in TTM-MD simulations of (001), (110), and (111) Cr targets irradiated with a $50 \mathrm{fs}$ laser pulse at an absorbed laser fluence of $800 \mathrm{~J} / \mathrm{m}^{2}$, which is $66 \%$ above the threshold for melting and solidification. The contour plots of the shear stresses are shown for the slip systems where the highest resolved shear stress is detected, (112)[11 $\overline{1}],(\overline{1} 12)[1 \overline{1} 1],(121)[1 \overline{1} 1]$, for (001), (110), (111) $\mathrm{Cr}$ targets, respectively. 
The results of the analysis of the resolved shear stress for the (111) target are not far from the ones discussed above for the (110) target, as can be seen from the contour plots of the temporal and spatial evolution of the resolved shear stress for the (211)[1 11] slip system, where the shear stress reaches its maximum value of $3.7 \mathrm{GPa}$, Fig. 3.15(c). In the (111) target the dislocations are emitted from the melting front at a point where the maximum resolved shear stress is reached after 20 ps from the laser pulse. At the time and depth of the dislocation emission, the lattice temperature is close to the melting temperature and the compressive pressure is around $3 \mathrm{GPa}$. Despite the pressure increase to $3.2 \mathrm{GPa}$ at depth of $32 \mathrm{~nm}$ below the surface by the time $50 \mathrm{ps}$, the resolved shear stress rapidly decreases to less than $1 \mathrm{GPa}$ in most of the slip systems except for six slip systems where the resolved shear stress is close to $1.3 \mathrm{GPa}$. Because of the slower propagation of the dislocations, the residual compressive stresses are partially relaxed only down to the depth of $115 \mathrm{~nm}$ below the surface, which is the maximum depth reaches by the dislocations by the end of the simulation.

In order to explain the appearance of dislocations in the (001) target in the spallation regime (Fig. 3.10) and their absence in the melting regime (Fig. 3.7), the results of the calculation of the resolved shear stresses in these two simulations are compared with each other in Table 3.2. The table illustrate that the maximum resolved shear stress is higher in several slip systems in the photomechanical spallation regime and reaches $3.1 \mathrm{GPa}$. This maximum value, however, is reached at a depth of $\sim 140 \mathrm{~nm}$ below the surface at the time when the lattice temperature at this location is close to $\sim 0.3 T_{m}$. As discussed above, in section 3.5.1, this maximum shear stress does not result in the homogeneous nucleation of dislocation loops. After the pressure wave leaves the surface region of the target, the residual stresses related to the uniaxial expansion of the target generate shear stresses that, in the vicinity of the melting front, reach the levels of $\sim 1.3 \mathrm{GPa}$ in the melting regime and $\sim 1.4 \mathrm{GPa}$ in the spallation regime. This higher shear stress, combined with other factors discussed in section 3.5.1, is likely to be responsible for the emission of dislocations in the spallation, but not the melting regime. 


\begin{tabular}{|c|c|c|c|c|c|c|c|}
\hline \multicolumn{2}{|c|}{ Slip systems } & \multirow{2}{*}{$\begin{array}{c}\begin{array}{c}\text { Max. RSS. } \\
\text { melting, GPa }\end{array} \\
0.02\end{array}$} & \multirow{2}{*}{$\begin{array}{c}\begin{array}{c}\text { Max. RSS. } \\
\text { spallation, GPa }\end{array} \\
0.1105\end{array}$} & \multicolumn{2}{|c|}{ Slip systems } & \multirow{2}{*}{$\begin{array}{c}\begin{array}{c}\text { Max. RSS. } \\
\text { melting, GPa }\end{array} \\
2.880\end{array}$} & \multirow{2}{*}{$\begin{array}{c}\begin{array}{c}\text { Max. RSS. } \\
\text { spallation, GPa }\end{array} \\
3.054\end{array}$} \\
\hline 1. & $(110)[\overline{1} 11]$ & & & 1. & $(123)[11 \overline{1}]$ & & \\
\hline 2. & $(110)[1 \overline{1} 1]$ & 0.02 & -0.110 & 2. & $(\overline{1} 23)\left[\begin{array}{ll}1 & 1\end{array}\right]$ & -2.883 & -3.053 \\
\hline 3. & $(1 \overline{1} 0)[111]$ & 0.014 & -0.106 & 3. & $(1 \overline{2} 3)[\overline{1} 11]$ & -2.880 & -3.055 \\
\hline 4. & $(1 \overline{1} 0)[11 \overline{1}]$ & 0.015 & -0.100 & 4. & $(12 \overline{3})[111]$ & 2.884 & 3.054 \\
\hline 5. & $(101)[11 \overline{1}]$ & 2.543 & 2.692 & 5. & $(132)\left[\begin{array}{lll}1 & 1 & 1\end{array}\right]$ & -1.925 & -2.036 \\
\hline 6. & $(101)[\overline{1} 11]$ & -2.542 & -2.694 & 6. & $(\overline{1} 32)[11 \overline{1}]$ & 1.919 & 2.037 \\
\hline 7. & $(10 \overline{1})[111]$ & 2.542 & 2.695 & 7. & $(1 \overline{3} 2)[111]$ & -1.924 & -2.035 \\
\hline 8. & $(10 \overline{1})[1 \overline{1} 1]$ & 2.543 & 2.692 & 8. & $(13 \overline{2})[\overline{1} 11]$ & 1.919 & 2.037 \\
\hline 9. & $(011)[11 \overline{1}]$ & 2.539 & 2.694 & 9. & $(213)[11 \overline{1}]$ & 2.881 & 3.053 \\
\hline 10. & $(011)[1 \overline{1} 1]$ & -2.544 & -2.693 & 10. & $(\overline{2} 13)[1 \overline{1} 1]$ & -2.883 & -3.053 \\
\hline 11. & $(01 \overline{1})[111]$ & 2.544 & 2.693 & 11. & $(2 \overline{1} 3)[\overline{1} 11]$ & -2.881 & -3.055 \\
\hline 12. & $(01 \overline{1})[\overline{1} 11]$ & 2.539 & 2.694 & 12. & $(21 \overline{3})[111]$ & 2.883 & 3.055 \\
\hline 13. & $(112)[11 \overline{1}]$ & 2.933 & 3.109 & 13. & $(231)[1 \overline{1} 1]$ & -0.965 & -1.019 \\
\hline 14. & $(\overline{1} 12)[1 \overline{1} 1]$ & -2.936 & -3.109 & 14. & $(\overline{2} 31)[11 \overline{1}]$ & 0.959 & 1.020 \\
\hline 15. & $(1 \overline{1} 2)[\overline{1} 11]$ & -2.933 & -3.111 & 15. & $(2 \overline{3} 1)[111]$ & -0.965 & -1.018 \\
\hline 16. & $(11 \overline{2})[111]$ & 2.936 & 3.110 & 16. & $(23 \overline{1})[\overline{1} 11]$ & 0.963 & 1.019 \\
\hline 17. & $(121)[1 \overline{1} 1]$ & -1.471 & -1.555 & 17. & $(312)[\overline{1} 11]$ & -1.923 & -2.037 \\
\hline 18. & $(\overline{1} 21)[11 \overline{1}]$ & 1.466 & 1.557 & 18. & $(\overline{3} 12)[111]$ & -1.922 & -2.038 \\
\hline 19. & $(1 \overline{2} 1)[111]$ & -1.471 & -1.554 & 19. & $(3 \overline{1} 2)[11 \overline{1}]$ & 1.924 & 2.035 \\
\hline 20. & $(12 \overline{1})[\overline{1} 11]$ & 1.467 & 1.556 & 20. & $(31 \overline{2})[1 \overline{1} 1]$ & 1.923 & 2.036 \\
\hline 21. & $(211)\left[\begin{array}{l}\overline{1} \\
11\end{array}\right]$ & -1.470 & -1.556 & 21. & $(321)[\overline{1} 11]$ & -0.964 & -1.019 \\
\hline 22. & $(\overline{2} 11)[111]$ & -1.467 & -1.557 & 22. & $(\overline{3} 21)[111]$ & -0.960 & -1.012 \\
\hline 23. & $(2 \overline{1} 1)[11 \overline{1}]$ & 1.472 & 1.554 & 23. & $(3 \overline{2} 1)[11 \overline{1}]$ & 0.967 & 1.018 \\
\hline 24. & $(21 \overline{1})[1 \overline{1} 1]$ & 1.471 & 1.556 & 24. & $(32 \overline{1})[1 \overline{1} 1]$ & 0.965 & 1.020 \\
\hline
\end{tabular}

Table. 3.2. The maximum values of the resolved shear stress calculated for the 48 slip systems in the (001) $\mathrm{Cr}$ targets. The $\mathrm{Cr}$ is irradiated by a $50 \mathrm{fs}$ laser pulse at two absorbed laser fluences, $800 \mathrm{~J} / \mathrm{m}^{2}$, which is $66 \%$ above the threshold for melting and solidification and $1100 \mathrm{~J} / \mathrm{m}^{2}$, which is slightly above the threshold for photomechanical spallation. All the values of the maximum resolved shear stress that are above $3 \mathrm{GPa}$ are marked with gray color. The $3 \mathrm{GPa}$ is lower than the theoretical resolved shear stress for Cr target, $3.8 \mathrm{GPa}$. 


\subsubsection{Generation of vacancies and self-interstitials in large-scale simulations of (001), (110), (111) Cr targets}

The analysis of the crystal defects in the three targets discussed above also includes the analysis of the point defects and provides an opportunity for investigation of the correlation between the concentration of vacancies and the crystallographic orientation of the irradiated targets. The visual inspection of the snapshots of the atomic configuration, where the defects are identified by the potential energies of the corresponding atomic configurations in Figs. 3.7, 3.11, and 3.12 for the (001), (110), and (111) Cr targets, respectively, reveals the generation of high densities of vacancies in the regions that experienced transient melting and solidification.

The analysis of the results of the three large-scale simulations discussed in this section also confirms these earlier observations. The application of the Crystal Analysis Tool to the final atomic configurations obtained after complete solidification of the three targets allows us to detect the individual vacancies and di-vacancies present in the surface regions of the targets. The vacancies distribution in the distribution of vacancies in the surface regions of the targets are show in Fig. 3.17, where the atoms are colored according to the type of the vacancy, the dark blue, green, and red represents cluster of atoms that surrounds an individual vacancy, di-vacancy that occupied first nearest neighbors, and di-vacancy occupied second nearest neighbors, respectively. 


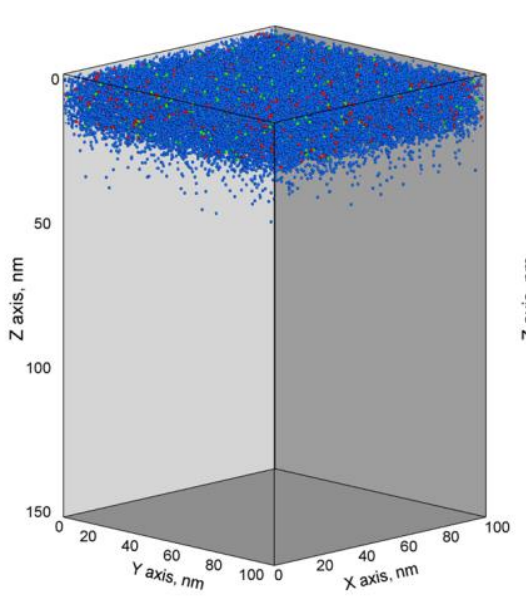

(001) $\mathrm{Cr}$



(110) $\mathrm{Cr}$

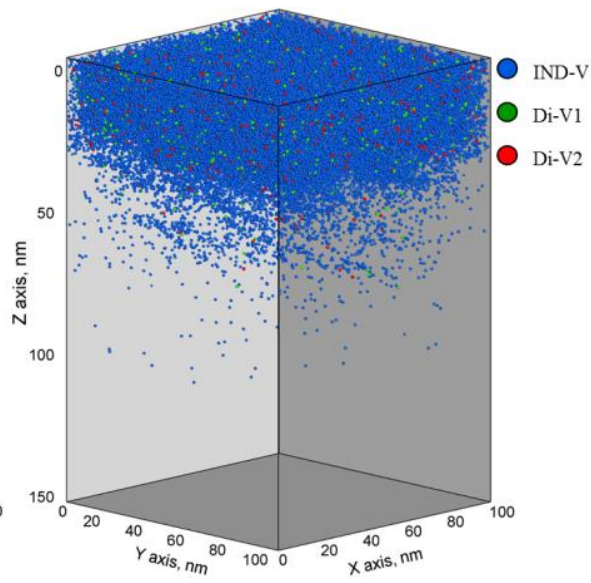

(111) $\mathrm{Cr}$

Fig. 3.17. Snapshots of the vacancy distributions in the surface regions of bulk (001), (110), and (111) $\mathrm{Cr}$ targets irradiated by a $50 \mathrm{fs}$ laser pulse at an absorbed laser fluence of $800 \mathrm{~J} / \mathrm{m}^{2}$, which is $66 \%$ above the threshold for the transition to the melting regime. The snapshots are taken at the end of the solidification process, $270 \mathrm{ps}$ after the laser pulse, and the atomic configurations are quenches to $0 \mathrm{~K}$ for $2 \mathrm{ps}$. The atoms are colored according to their type, blue atoms surrounded the individual vacancy, green atoms surrounded the di-vacancy occupied the first nearest neighbor, and red atoms surrounding the divacancies occupied the second nearest neighbor.

The concentration of vacancies with respect to the distance from the surface is shown for the three targets in Fig. 3.18. Three distinct regions are marked by color in the figure. The light blue regions represent parts of the targets that experienced melting and solidification. The narrow blue regions represent the overheated crystalline layers adjacent to the resolidified region.

This region may experience a transient appearance of several liquid islands generated in the immediate vicinity of the melting front and is defined from the calculation of the liquid fraction as a function of depth, which yields the thickness of the intermediate liquid - solid region of 4, 6 , and $4 \mathrm{~nm}$ for the (001), (110), and (111) targets, respectively. Finally, the gray region represents the rest of the crystal. The results illustrate the generation of a high concentration of the individual vacancies in the region that experienced melting and solidification process in the three targets. The average concentrations of vacancies in the melting regions are represented by red dashed lines in Fig. 3.18, and are very similar in the three targets $0.38 \%, 0.37 \%$, and $0.36 \%$ in the (001), (110), and (111) targets, respectively. The vacancy concentrations predicted in the three large-scale simulations are also close to the ones obtained in small-scale simulation for 
(001) Cr target irradiated with a $200 \mathrm{fs}$ laser pulse at an absorbed laser fluence $850 \mathrm{~J} / \mathrm{m}^{2}$, see Fig. 3.3. In contrast to the high concentration of individual vacancies, the concentration of divacancies, either occupying the first or the second nearest neighbor lattice sites, remain at much lower levels of $\sim 0.02 \%$, in the three targets. The low concentration of di-vacancies may be related to the high formation energy of di-vacancies. According to the Density-function calculation, Ref.[199], the formation energy of di-vacancies for $\mathrm{Cr}$ is equal to $E_{f(1 N N)}^{2 v} \approx 3.34-5.01 \mathrm{eV}$ and $E_{b(2 N N)}^{2 v} \approx 3.35-5.16 \mathrm{eV}$, which is higher than the vacancy formation energy $E_{f}^{v} \approx 2.64 \mathrm{eV}$ in $\mathrm{Cr}$ target.

The close values of the concentrations of individual vacancies in the three targets indicate that the concentration of vacancies is not strongly influenced by the crystallographic orientation of the targets. As discussed in the next section, the generation of vacancies in the process of laser-induced melting and resolidification takes place at the rapidly advancing solidification front and correlates with the velocity of the solidification front propagation. The results of MD simulations performed for several bcc metals indicate that the velocity of solidification front is almost the same in the three crystallographic directions, [001], [110], and [111] [206]. Accordingly, as the velocities of the solidification front in the three large-scale TTM-MD simulations ranged between $\sim 100$ and $130 \mathrm{~m} / \mathrm{s}$, the similar concentrations of vacancies in the three targets are not surprising. 

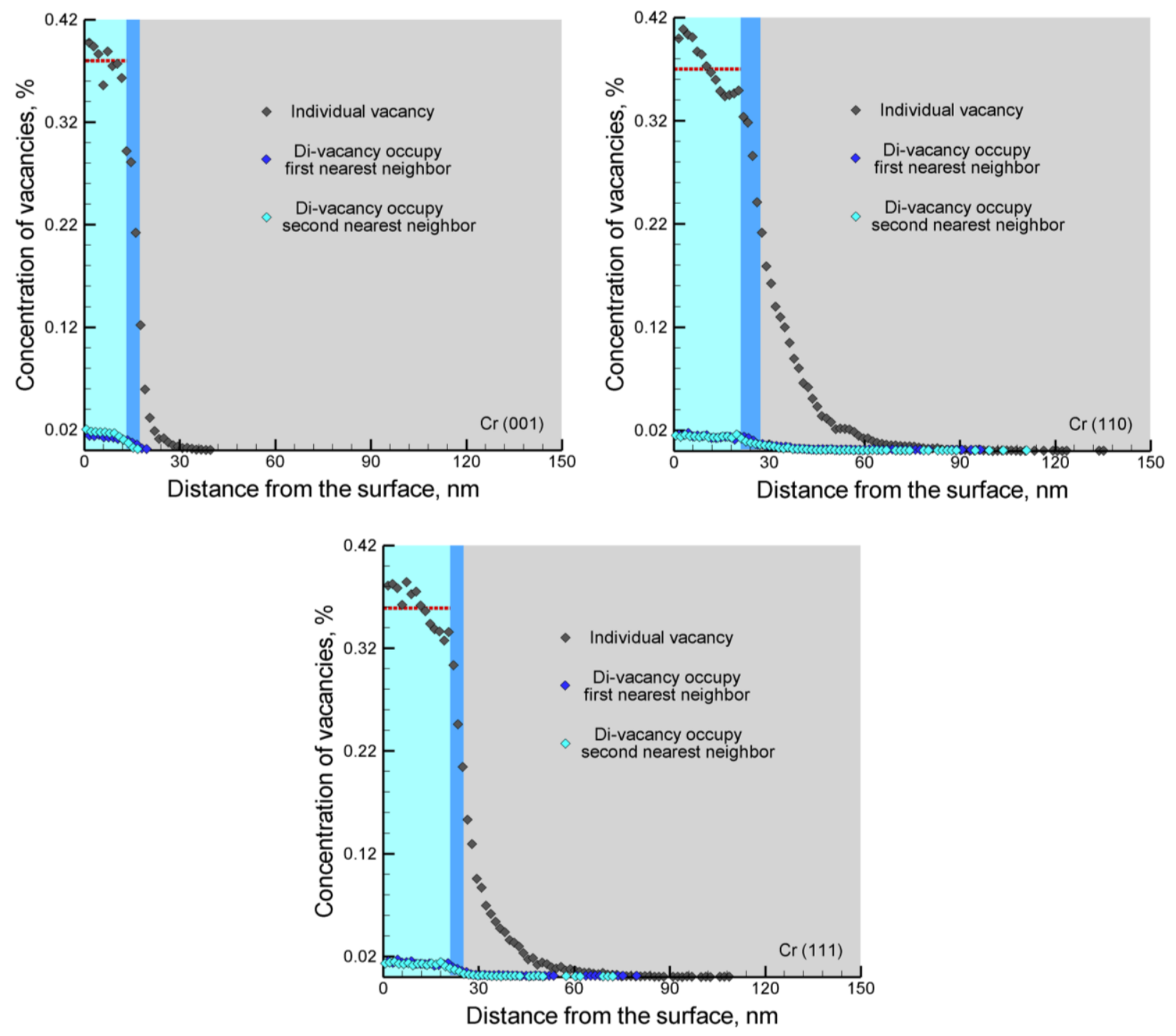

Fig. 3.18. Concentration of vacancies in the surface regions of (a) (001), (b) (110), and (c) (111) $\mathrm{Cr}$ targets irradiated with $50 \mathrm{fs}$ laser pulses at absorbed laser fluences of $800 \mathrm{~J} / \mathrm{m}^{2}$. The gray, dark blue, and light blue diamond symbols represent the concentration of individual vacancies, di-vacancies occupying the first nearest neighbor lattice sites, and di-vacancies occupying the second nearest neighbor lattice sites. The light blue, narrow blue, and gray parts of the plot represent a region that experienced melting and solidification, an intermediate layer, and the rest of the target, respectively. The red dashed lines mark the average concentrations of the individual vacancies in the melting and solidification regions. 


\subsection{Summary}

The analysis of the results obtained from the TTM-MD simulations of $\mathrm{Cr}$ targets irradiated by a 200 fs laser pulse at a broad range of absorbed laser fluences and discussed in section 2.3.2, reveals the formation of a high density of point defects, mostly vacancies with some self-interstitials, in the region that experienced transient melting and solidification. The generation of the high densities of vacancies may have important implications on the physical, chemical, and mechanical properties of the surface layers. The variation of the vacancy concentration with depth under the irradiated surface is analyzed for each simulation and is found to be almost constant within the resolidified regions. The values of the vacancy concentration are ranging between $0.42 \%$ and $0.47 \%$, much higher than the equilibrium concentration of vacancies at the melting temperature. As discussed in the next section, the high concentrations of vacancies in the surface regions of the irradiated targets are related to the fast propagation of the solidification front under conditions of strong undercooling, when the surface temperature drops down to $0.80 T_{m}$ and $0.88 T_{m}$ by the end of the simulations.

The concentrations of vacancies are also determined for bulk Ni targets with fcc crystal structure, in order to compare the results with the ones obtained for the bcc $\mathrm{Cr}$ targets. The analysis is carried out for atomic configurations generated in earlier simulations for Ni targets irradiated by a 1 ps laser pulse at a range of absorbed laser fluences covering the same three irradiated regimes as in the simulations for $\mathrm{Cr}$. The results also reveal the generation of high densities of vacancies in the regions of the targets that experienced melting and resolidification. The value of the vacancy concentration of vacancies is ranging between $0.036 \%$ and $0.058 \%$ for the $\mathrm{Ni}$ targets and the surface temperature at the end of the simulations is between $0.81 T_{m}$ and $0.86 T_{m}$

Following the analysis of the point defects, the investigation is extended to the generation of the dislocations and, in particular, the dependence of the dislocation emission on the crystallographic orientation of the irradiated target. Accordingly, three large-scale TTM-MD simulations Cr targets with (001), (110), and (111) surface orientations are performed for systems irradiated by a $50 \mathrm{fs}$ laser pulse at an absorbed laser fluence of $800 \mathrm{~J} / \mathrm{m}^{2}$, which is above the threshold for transition to the melting and solidification regime. For (001) Cr target, the generation of unstable stacking faults is found to take place during the uniaxial expansion in the 
direction normal to the irradiated surface. The region affected by the generation of the stacking faults expands with the propagation of the tensile component of the laser-induced stress wave and shrinks and disappear when the tensile wave leaves the surface region and the lattice deformation is partially relaxes. For (110) and (111) Cr targets, the emission of a high density of perfect dislocations from the melting front is observed. The calculation of the resolved shear stress for the 48 slip systems for the three simulations elucidate that the high value of the resolved shear stress in (110) and (111) targets at a depth that is just below the melting front, along with the high lattice temperature, are the reasons for the emission of the dislocations. The absence of the perfect dislocations in the (001) target may be explained by the relatively low values of the resolved shear stress near the melting front. In addition to the dislocations, the formation of high densities of point defects, between $0.36 \%$ and $0.38 \%$, is also observed in the large-scale simulations. 


\section{Chapter 4}

\section{Generation of point defects by rapid solidification of} $\mathrm{Ni}$ and $\mathrm{Cr}$ under conditions of strong undercooling 


\subsection{Introduction}

Solidification process has been in the core of materials science studies since the last century due to its importance to many aspects of materials fabrication and processing [200].In the area of laser-materials interactions, the solidification process plays an important role in creating surface nanostructured materials by changing the structure and composition of the surface layer through the formation of metastable phases, grain refinement, generation of crystal defects and the redistribution of the alloying elements. The original thoughts on the solidification process date back to Wilson [201] and Frenkel [202] who proposed that the crystal growth process is a thermally activated process due to its dependence on the mobility of atoms in the liquid phase.

For pure metals, the solidification process can be divided into two sequential steps, nucleation and growth. The first step mainly depends on the time necessary for the formation of a crystal with a certain critical size by thermal fluctuations, while the second step relies on the crystal growth mechanism which is largely defined by whether the liquid-crystal interface is atomically rough or atomically flat. Most of the experimental studies on this topic are limited to the liquid-solid phase transformation close to the equilibrium melting point. The reason for this is probably related to the difficulties of avoiding the nucleation of crystalline structures under the conditions of strong undercooling. Even with the successful elimination of the heterogeneous nucleation in small "non-communicating" liquid droplets by Turnbull, the liquid metals cannot be undercooled below $\sim 0.8 T_{m}$ due to the onset of rapid homogeneous nucleation [200]. Although years have been spent on understanding the classical nucleation theory and defining the microscopic parameters that control the nucleation phenomenon, the understanding of the microscopic mechanisms that control the movement of the liquid-solid interface far from the melting temperature is still rather poor. For instance, the nature of the atomic rearrangements resulting in the advancement of the solidification front remains unclear and requires additional investigations. A related question on the mechanisms responsible for the generation of vacancies at the rapidly advancing solidification front also remains unexplored.

Computational modeling and, in particular, atomistic molecular dynamics simulations can provide answers to some of these important questions. In this chapter, MD modeling is used 
for investigation of the mechanisms responsible for the vacancy formation during the rapid solidification process in two systems with different crystal structures, bcc and fcc. Molecular dynamics simulations are performed for liquid-solid coexistence systems under well-controlled temperature and pressure conditions. The vacancies are detected in each atomic plane and the concentration of vacancies in each simulation is related to the solidification front velocity. The diffusivity of vacancies and the time between vacancy jumps are calculated for both system at different temperatures and used in the interpretation of the simulation results. The effect of the nucleation of new crystallites inside the undercooled liquid phase on the velocity of the solidification front is also investigated for $\mathrm{Ni}$ system represented by different interatomic interaction potentials.

\subsection{Computational model}

In general, two mechanisms may be responsible for the generation of vacancies in metal target irradiated by a short laser pulse: (1) thermally activated generation of Frenkel pairs in the crystalline part of the system and (2) the formation of vacancies at a rapidly progressing solidification front during the solidification process. In order to identify the mechanism that may play the dominant role in the laser-induced generation of point defects, series of liquid-solid MD simulations are performed for EAM Cr and EAM Ni systems.

The dimensions of $\mathrm{Cr}$ computational system are $3 \times 3 \mathrm{~nm}^{2}$ for the lateral $\mathrm{x}$ and $\mathrm{y}$ directions and $100 \mathrm{~nm}$ along z-axis, and the system is composed of 70000 atoms. In Ni system, the computational cell has dimensions of $5.4 \times 5.4 \times 54 \mathrm{~nm}^{3}$ and the total number of atoms equal to 135000 . Initially, the two systems are thermalized at $300 \mathrm{~K}$ for $200 \mathrm{ps}$, and then the temperature of three quarters of each system is increased to above the melting temperature by scaling the atomic velocities to create the liquid phase region in the system. Meanwhile, the temperature of the rest of the atoms in the computational cell is maintained slightly below the melting temperature, so that this part of the system stays in the solid phase. The liquid-crystal coexistence system is then relaxed at the equilibrium melting temperature and quenched (quickly cooled) to the desired temperature below the melting temperature. A schematic diagram of the thermal treatment of the samples used in the simulations of the solidification process is shown in Fig. 4.1. 


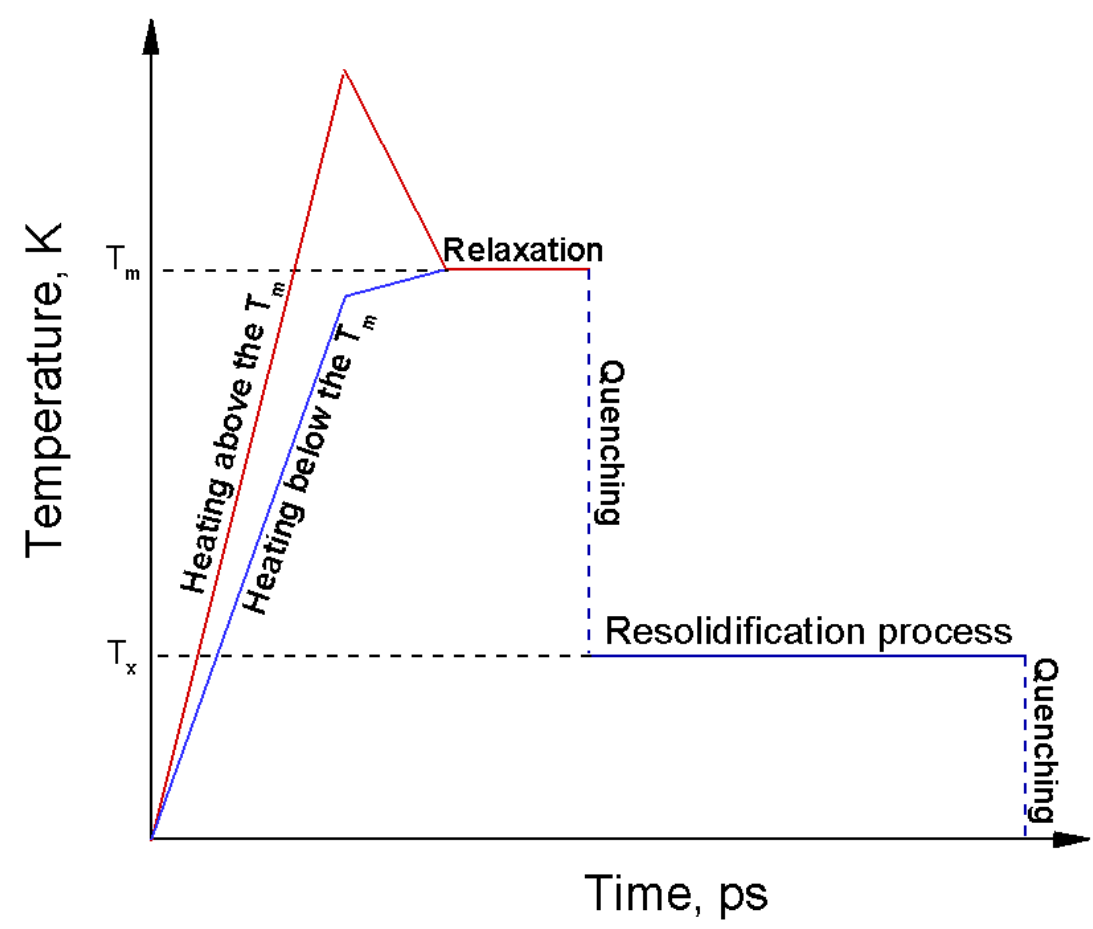

Fig. 4.1. Schematic diagram of the thermal treatment of $\mathrm{Cr}$ and $\mathrm{Ni}$ systems that is used in the MD simulation study of the mechanisms responsible for the formation of vacancies under conditions of fixed undercooling. The red line represents the three quarters of the system that is heated above $T_{m}$ while the blue one represents the remaining of the system with temperature below $T_{m}$.

Once the samples are prepared, the simulations of solidification under controlled temperature and pressure conditions are performed. In order to avoid the local increase in the temperature at the rapidly advancing interface due to the release of heat of melting, the systems are divided into 30 layers with a thickness of $3 \mathrm{~nm}$ for $\mathrm{Cr}$ and $1.8 \mathrm{~nm}$ for $\mathrm{Ni}$ and the average local temperature is controlled for each layer. This method allows us to investigate the mechanism of vacancy formation under the condition of fixed undercooling. In total, 16 simulations for the EAM Cr system and 23 simulations for the EAM Ni system are performed at different specific undercooling temperatures and constant zero pressure. The range of temperatures explored in the simulations performed for EAM $\mathrm{Cr}$ is from $1.00 T_{m}$ to $0.20 T_{m}$, with the temperature increment equal to $0.05 T_{m}$. For the EAM Ni, the temperature range is from $1.00 T_{m}$ to $0.01 T_{m}$ and the temperature increment is equal to $0.05 T_{m}$ down to $0.05 T_{m}$ and $0.01 T_{m}$ between $0.05 T_{m}$ and $0.01 T_{m}$. The interatomic interaction in these simulations is described by the EAM potential in the form and parameterization developed for Cr in Ref. [129] and for Ni in Ref. [182]. 
The melting temperature for the two metals is obtained from a series of simulations performed for liquid-solid systems under conditions of constant pressure and temperature. The melting temperatures for both systems are determined when the solidification front velocity reaches zero, and it is equal to $2332 \mathrm{~K}$ for EAM Cr [134], and $1445 \mathrm{~K}$ for EAM Ni. To provide a clear view of the vacancies formation in the system after the complete solidification, the same visualization method that is used in section 2.3.3 is used in this work. Lastly, in order to define the error bars which represent the standard deviation for the concentration of vacancies, we performed three sets of the 16 and 23 simulations for the two metals, where each set started with different initial conditions.

\subsection{Results and discussion for (001) interfaces}

The mechanisms of the vacancy formation at a rapidly advancing solidification front are discussed in this section based on the results obtained in a series of MD simulations performed for EAM Cr with bcc crystal structure and EAM Ni with fcc crystal structure.

\subsubsection{Generation of vacancies in $\mathrm{Cr}$ and $\mathrm{Ni}$ during a solidification process}

After creating the coexisting liquid and solid phases in the $\mathrm{Cr}$ and $\mathrm{Ni}$ systems as explained above, two different methods are used to stabilize the solidification front at the melting temperatures. For EAM Cr, the creation of the liquid phase at temperature $T_{l}=2400 \mathrm{~K}$ and the solid phase at temperature $T_{s}=2200 \mathrm{~K}$ results in establishment of a non-equilibrium system. In order to acquire the liquid-solid coexistence condition, the system passes through an adiabatic process under a constant pressure. This process allows for cooling of the liquid phase, and heating of the solid phase so that the temperature of the system gradually reaches the equilibrium melting temperature and the velocity of the liquid-crystal interface approaches zero. During this adiabatic process, $\sim 26 \mathrm{~nm}$ thick layer is transferred from the liquid to the solid phase resulting in the formation of some vacancies before quenching the system to the desirable temperature. This region is excluded from the analysis of the vacancy concentration in the solidified system. In contrast to $\mathrm{Cr}$, the simulations for EAM Ni are performed for systems prepared under conditions of constant zero pressure and melting temperature. The velocity of the solidification front is changing in these simulations from negative to positive values, depending on the temperature, and the equilibrium melting temperature of the EAM Ni system is determined as the temperature 
that produces the solidification front velocity approximately equal to zero. After the melting temperature is determined, the system with approximately zero solidification front velocity is equilibrated at the melting temperature, $T_{m}=1445 \mathrm{~K}$, and then quenched to different desirable undercooling temperature below $T_{m}$. This process is considered to be more favorable than the one used in the $\mathrm{Cr}$ simulations because it yields a well-defined region where the solidification took place under desired undercooling condition.

The visual inspection of snapshots of atomic configurations taken at different simulation times serves as a starting point in the analyses of the formation of vacancies under the predefined undercooling conditions. Figure 4.2 provides two examples of MD simulations for $\mathrm{Cr}$ and Ni systems where the two systems are exposed to an undercooling temperature of $0.75 T_{m}$. To produce these snapshots, the systems are quenched to $0 \mathrm{~K}$ for $1 \mathrm{ps}$ in order to reduce the thermal noise in the atomic positions and energies, as explained previously. In addition, the atoms that belong to the original bcc and fcc crystal structure with energies below $-3.99 \mathrm{eV}$ for EAM Cr and $-4.35 \mathrm{eV}$ for EAM Ni are blanked while all the atoms with higher potential energies are shown in these snapshots. This approach provides a clear view of the liquid phase and the crystal defects generated in the solidified parts of the systems.

Similar to the snapshots discussed in section 3.3, each vacancy in $\mathrm{Cr}$ appears as a cluster of 14 atoms representing the first and second nearest neighbor shells around the vacant lattice site, whereas in Ni each individual vacancy is represented by a cluster of 12 atoms of the first nearest neighbor shell around the vacancy site. The dashed black rectangles correspond to the regions where the two solidification fronts meet each other and create a region with high density of defects. Accordingly, these regions are excluded from the calculation of the vacancy concentration. Ultimately, after completing the solidification process, the number of vacancies in each atomic plane is calculated and plotted as represented in Fig. 4.3. In this figure, each bar in the histogram represents the average of ten individual (001) atomic planes. The analysis of the results obtained in the MD simulations indicates that almost all the vacancies in both systems are located in the regions that had experienced solidification process. In the simulation of the EAM Cr system, the formation of some vacancies at $\sim 0.9 \mathrm{~nm}$ and between $78 \mathrm{~nm}$ to $84 \mathrm{~nm}$ is related to the high initial temperature of the liquid phase, which resulted in the movement of the interface in the direction opposite to the solidification process before the steady-state solidification process is established. 

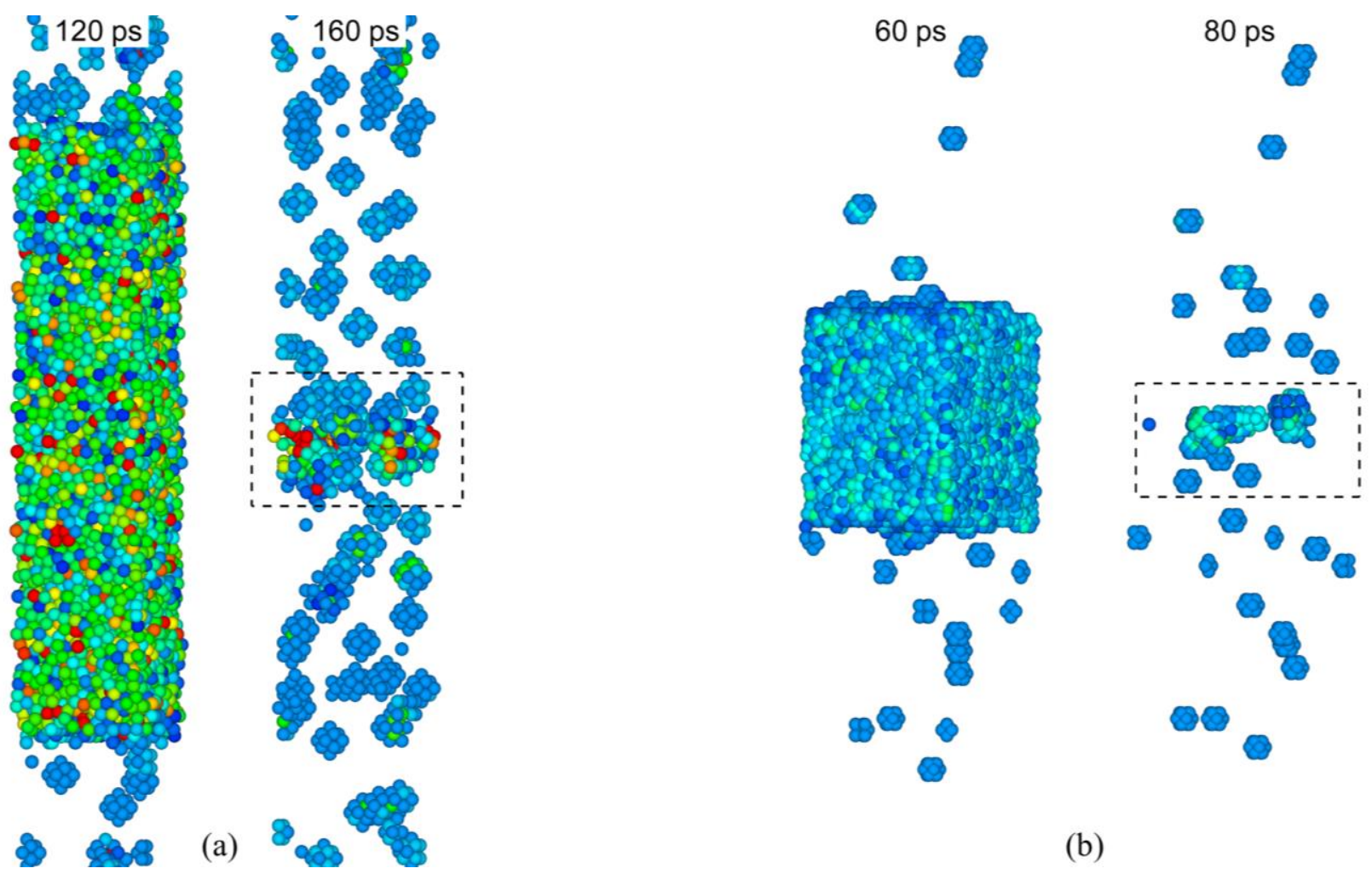

(b)

Fig. 4.2. Snapshots of the atomic configurations obtained in MD simulations of the solidification of liquid-crystal EAM Cr (a) and EAM Ni (b) systems. After the equilibration at the melting temperature, the two systems are rapidly cooled to $0.75 T_{m}$ and the snapshots are shown for two times during the constant-temperature solidification process. The configurations are quenched to $0 \mathrm{~K}$ for $1 \mathrm{ps}$ in order to reduce thermal noise in the atomic positions and energies, and to provide a clear view of the vacancies remaining in the system. The atoms are colored according to their potential energies and all atoms that have low potential energy and belong to bcc or fcc crystal structure are blanked. Only the atoms with potential energy higher than $-3.99 \mathrm{eV}$ for EAM $\mathrm{Cr}$ and $-4.35 \mathrm{eV}$ for EAM Ni are shown in the snapshots. The dashed black rectangles in (a) and (b) represent the regions where the two crystallization fronts meet each other. The snapshots are taken along the $[1 \overline{1} 0]$ view direction. The time in each snapshot represents the time after quenching to the required temperature. In (a), an individual vacancy is represented by a cluster composed of 14 atoms that includes the first eight and the second six nearest neighbors of the empty lattice site. In (b), an individual vacancy is represented by a cluster of 12 atoms that include the first neighbor shell surrounding the vacancy site. 

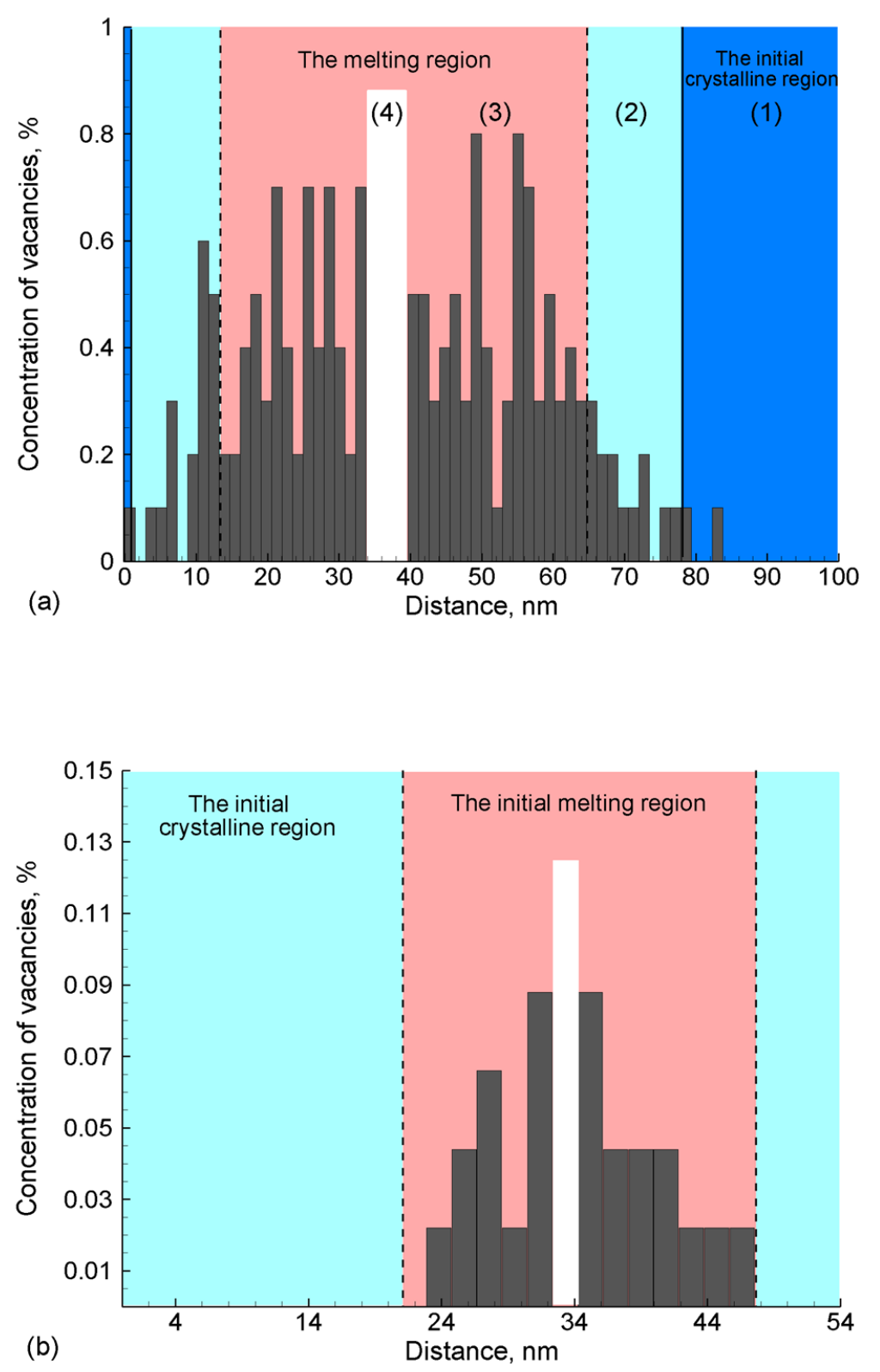

Fig. 4.3. Distribution of vacancies in the EAM Cr (a) and EAM Ni (b) systems solidified at a temperature of $0.75 T_{m}$. Each bar in the two histograms represents the vacancy concentration averaged over ten individual (001) atomic planes. In (a), the dark blue region, (1), marks the solid part of in the initial EAM $\mathrm{Cr}$ system, and the light blue regions, (2), correspond to the parts that solidified during the adiabatic process used for the generation of liquid-solid coexistence state at $T_{m}$, before quenching the system to the desired temperature. Regions (2) and (3) and (4) represent the liquid phase in the initial liquid-solid $\mathrm{Cr}$ system. In (b), light blue region represents the initial solid phase in the liquid-solid EAM Ni system and the light pink region represents the initial liquid phase. In the two systems, the white region, which is also marked as (4) in (a), corresponds to the region where the two solidification fronts meet each other. The solid black lines in (a) represent the initial positions of the two interfaces during the formation of the liquid-solid system, while the dashed lines in (a) and (b) represent the positions of the two interfaces at the liquid-solid coexistence condition, before quenching the system to the desired temperature. 


\subsubsection{Effect of the solidification front velocity on the concentration of vacancies}

The analysis of the vacancy formation in EAM $\mathrm{Cr}$ and EAM Ni includes the results obtained from simulations performed in a temperature range between $0.95 T_{m}$ and $0.20 T_{m}$ for $\mathrm{Cr}$ and between $0.95 T_{m}$ and $0.05 T_{m}$ for Ni. For each temperature, the average vacancy concentration in the solidified parts of the system and the steady-state solidification front velocity are calculated and is plotted with respect to the normalized temperature in Figs. 4.4 and 4.5 for EAM $\mathrm{Cr}$ and $\mathrm{EAM} \mathrm{Ni}$, respectively.

In the EAM Cr simulations, the behavior of solidification front velocity as a function of temperature can be divided into two regions. The first region corresponds to the rapid increase of the velocity of the solidification front with increasing undercooling and extends from the melting temperature down to $0.75 T_{m}$, while the second region, below $0.75 T_{m}$, is characterized by a gradual decrease of the front velocity. This behavior can first be discussed in terms of the basic thermodynamics and kinetics concepts. In general, the movement of the liquid-solid interface is controlled by the rearrangement of the atoms from the liquid to the solid phases where the liquid phase is considered to be the source and the sink for the defects in the system [203]. After the equilibration of the liquid-solid system at the melting temperature, the free energies of both phases are equal and the system is in the coexistence state. As the system is brought to a state of undercooling by $\Delta T$ below $T_{m}$, the free energy of the two phases are changed in a way that the free energy of the solid phase becomes lower than the one of the liquid phase. Thus, undercooling condition creates the driving force for the solidification process.

At a high temperature, close to the melting point, the system still acts as if in a quasiequilibrium state. Furthermore, the driving force for the crystallization at this high temperature is very sensitive to the variation of temperature. This is in contrast to the atomic mobility, which is not highly affected by the small changes in the temperature values. Due to the high thermal energy of the atoms in this region, the simulations performed for temperatures of $0.95 T_{m}$ and $0.90 T_{m}$, take longer time, between $230 \mathrm{ps}$ and $336 \mathrm{ps}$, in order to complete the solidification process as compared, for instance, to the time of the solidification process in the simulation at $0.75 T_{m}, \sim 160 \mathrm{ps}$. The results illustrate that the velocity of the solidification front at $0.95 T_{m}$ and $0.90 T_{m}$ are $69 \mathrm{~m} / \mathrm{s}$ and $103 \mathrm{~m} / \mathrm{s}$, respectively, while the concentration of vacancies stays at a constant level of about $0.49 \%$ Fig. 4.4 . 

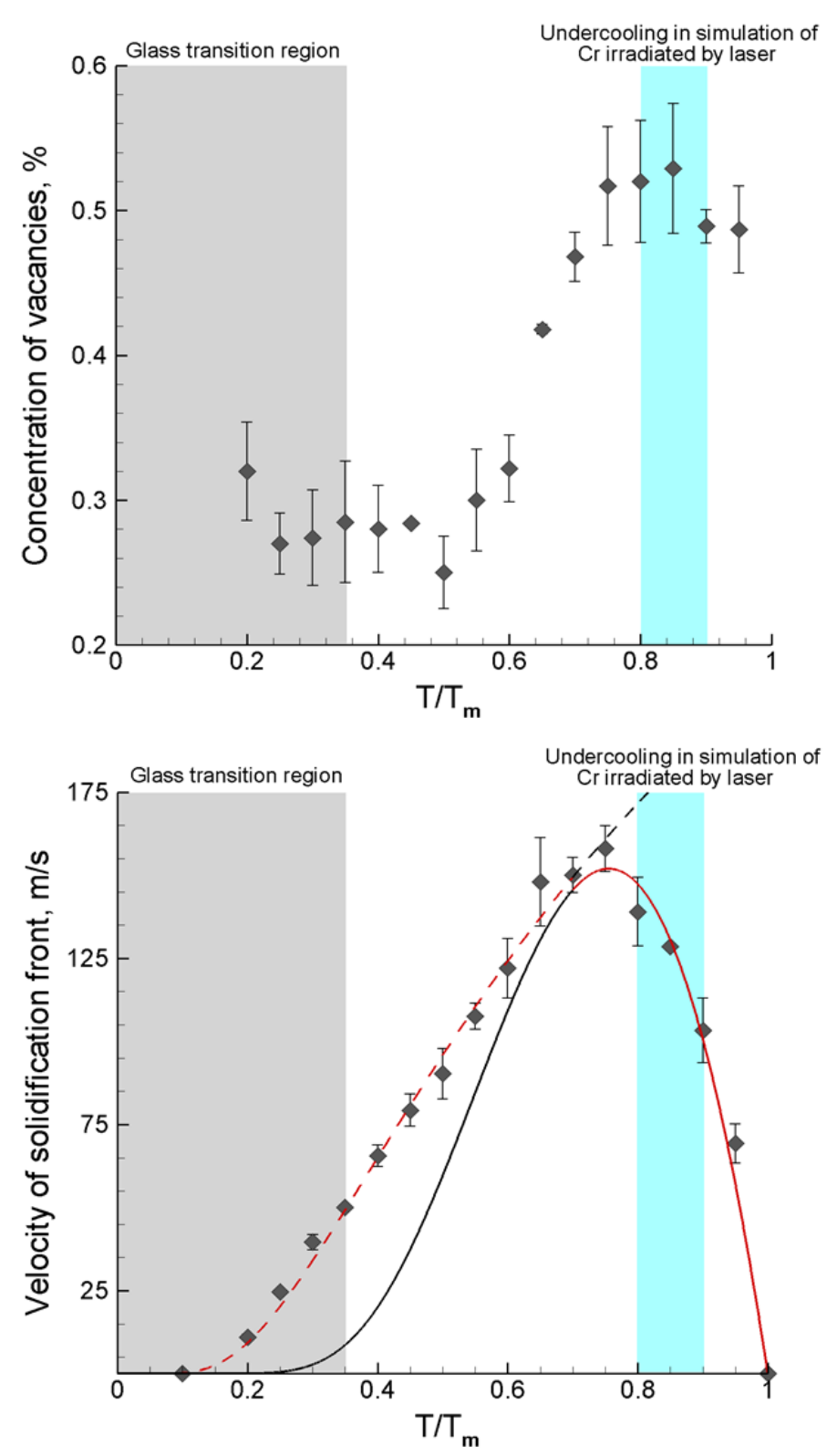

Fig. 4.4. Concentration of vacancies and velocity of solidification front as functions of normalized temperature in EAM Cr system obtained in a series of MD simulations performed at different levels undercooling below the equilibrium melting temperature. The light blue region in both figures corresponds to the undercooling conditions realized in the simulations of laser interactions with the $\mathrm{Cr}$ targets. The gray region represents the amorphous state of the EAM Cr material, where the glass transition temperature is found to be $T_{g}=810 \mathrm{~K}$. The error bars represent the standard deviations of the concentration of vacancies and the solidification front velocity in the upper and lower figures, respectively. The error bars in both cases are obtained from the results of three sets of simulations, each starting at different initial conditions. The red solid line represents the fitting of the MD results to Eq. (21), while the dashed red line represents the fitting of the MD results to Eq. (22). The black solid and dashed lines represent regions where Eqs. (21) and (22) do not match the results of the simulations. 
At lower temperatures, in the range from $0.85 T_{m}$ to $0.75 T_{m}$, the velocity of the solidification front increases from $129 \mathrm{~m} / \mathrm{s}$ up to the maximum value of $158 \mathrm{~m} / \mathrm{s}$, while the concentration of vacancies experiences only a moderate increase to about $0.52 \%-0.53 \%$.

Progressively, with increasingly stronger undercooling (and the corresponding increase in the driving force for the solidification), we observed a decrease in the velocity of solidification front, along with a sharp drop in the concentration of vacancies. This behavior can be explained by two main factors: changes in the atomic mobility, and the homogeneous nucleation of new crystallites inside the undercooled liquid phase. Undoubtedly, the atomic mobility is directly affected by the reduction of temperature, which results in the deceleration of the solidification front velocity. Consequently, the atoms near the interfacial region from the liquid side have more time to move and occupy correct lattice sites in the bcc crystal structure. Moreover, some of the atoms that join the solid phase can diffuse inside the solid and annihilate some of the vacancies that are left behind the solidification front, as demonstrated in section 4.3.3 below, which eventually results in the reduction of the concentration of vacancies.

The homogeneous nucleation of new crystallites within the liquid regions can also play a significant role in the deceleration of the liquid-solid interface, as explained in more detail in section 4.4. We observe homogeneous nucleation of small solid structures inside the liquid phase in $\mathrm{Cr}$ simulations for $\mathrm{Cr}$, at $0.60 T_{m}$ and $0.65 T_{m}$, where the new crystallites grow and combine together during the solidification process. Ultimately, the formation of the solid phase inside the liquid impedes the advancing of the interface and reduces the solidification front velocity. Due to the differences in the mechanisms of the vacancy formation in the regions of the homogeneous nucleation and the ones that solidified through the rapid movement of the solidification front, we exclude the regions of the homogeneous nucleation from the calculations of the vacancy concentrations. With the continued increase of the driving force but decreasing atomic mobility, the velocity of the solidification front is further reduced for temperatures between $0.55 T_{m}$ and $0.20 T_{m}$ from $107 \mathrm{~m} / \mathrm{s}$ to $11 \mathrm{~m} / \mathrm{s}$, while the concentration of vacancies continue to fluctuate between $0.30 \%$ and $0.32 \%$.

The analysis of the solidification front velocity is also expanded to include a simulation at $0.10 T_{m}$. Although the simulation takes more than $10 \mathrm{~ns}$, the position of the solidification front reveals that the velocity of the interface is approximately zero. This result indicates that the 
liquid phase in this simulation is converted to the amorphous glass structure and the crystalliteglass interface remains stable at this temperature. We determined the glass transition temperature for the EAM Cr system by method used in Ref. [204]. Consequently, a series of MD simulations for EAM $\mathrm{Cr}$ liquid system is performed under well controlled temperature and pressure conditions for a system with a total number of atoms of 6750 . The temperature dependence of the second moment of the atomic-level hydrostatic stress distribution is calculated in the simulations and the glass transition temperature is determined from the intersection of the linear extrapolations of the dependences for the liquid phase and amorphous structure. This approach yields glass transition temperature of $810 \mathrm{~K}$, which is equal to $0.35 T_{\mathrm{m}}$.

Turning to the EAM Ni system and following the same method that is used to analyze the results for the EAM Cr system, the concentration of vacancies and the velocity of solidification front are obtained from a series of MD simulations of the liquid-solid Ni system and plotted as functions of normalized temperature in Fig. 4.5. In general, the curve of the solidification front velocity for the Ni system shows both similarities and differences from the one discussed above for $\mathrm{Cr}$. The overall picture of the solidification front velocity curve can be described as follows: At temperatures near the melting point down to $0.80 T_{m}$, the results indicate an approximately linear dependence of the solidification front velocity on temperature, with the velocity increasing from zero up to $171 \mathrm{~m} / \mathrm{s}$. At lower temperatures, between $0.10 T_{m}$ and $0.70 T_{m}$, the interface velocity remains nearly constant at a level of $164 \mathrm{~m} / \mathrm{s}-174 \mathrm{~m} / \mathrm{s}$. Eventually, the velocities values decrease from $167 \mathrm{~m} / \mathrm{s}$ to $137 \mathrm{~m} / \mathrm{s}$ in simulations performed at temperatures ranging between $0.05 T_{m}$ and $0.01 T_{m}$

The temperature-independence of the interface velocity observed for a wide range of temperatures, Fig. 4.5, for Ni described by "Johnson EAM potential" [182] is larger confirmed in additional simulations performed with a different EAM potential in the form proposed by Foiles, Baskes, and Daw, “EAM-FBD” [128]. The quantitative differences between the results obtained with the two potentials are largely defined by the higher propensity for homogeneous nucleation of crystallites in the supercooled liquid described by the EAM-FBD potential as compared to the Johnson EAM potential. 

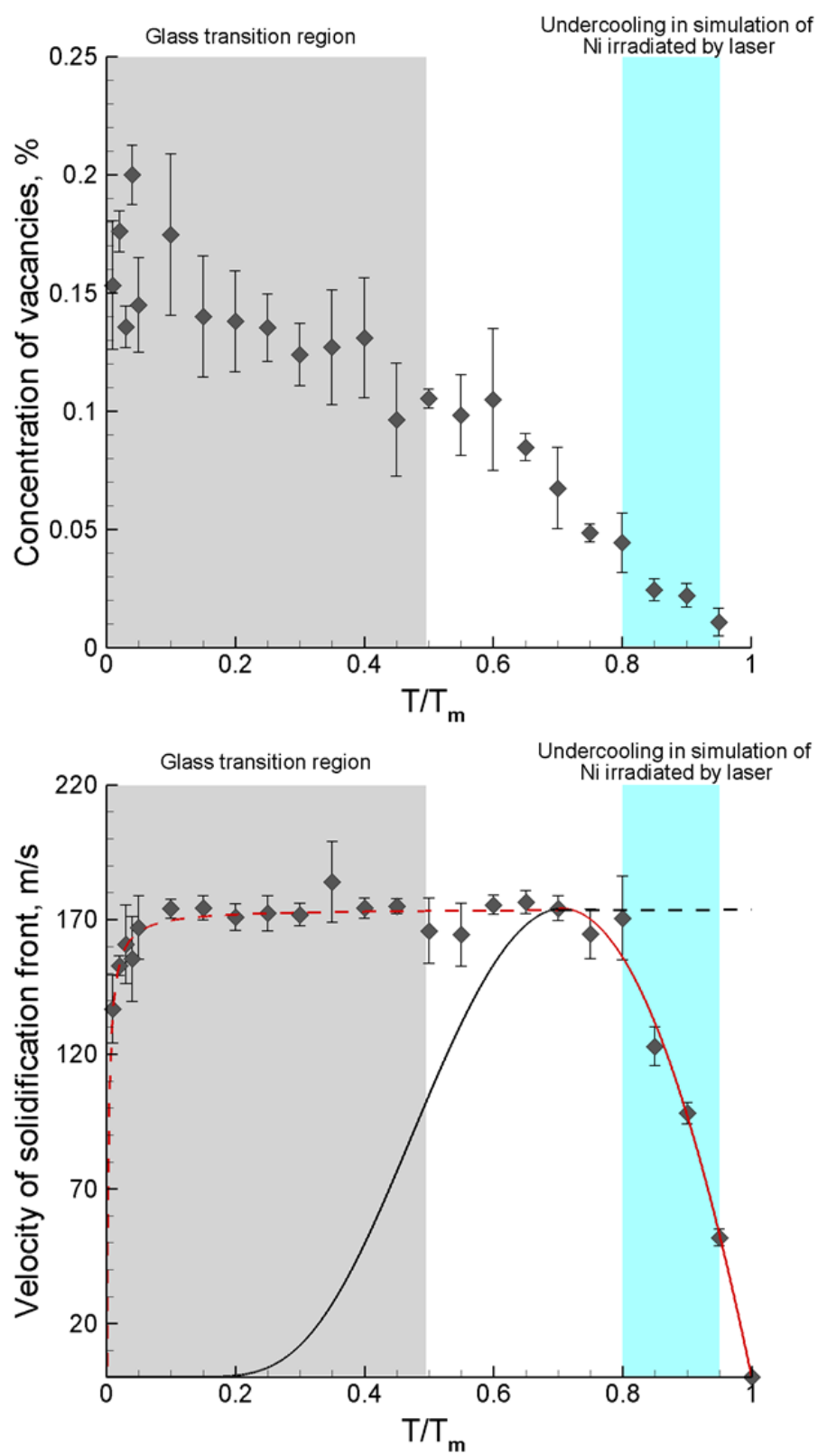

Fig. 4.5. Concentration of vacancies and velocity of solidification front as functions of normalized temperature in EAM Ni system obtained in a series of MD simulations performed at different levels of undercooling below the equilibrium melting temperature. The light blue regions in both figures correspond to the undercooling conditions realized in the simulations of laser interactions with Ni targets. The gray region represents the amorphous state of the EAM Ni material, where the glass transition temperature is $T_{g}=717 \mathrm{~K}$ according to Ref. [179]. The error bars represent the standard deviation of the concentration of vacancies and the solidification front velocity in the upper and lower figures, respectively. The error bars in both cases are obtained from the results of three sets of simulations, each starting with different initial conditions. The red solid line represents the fitting of the MD results to Eq. (21), while the dashed red line represents the fitting of the MD results to Eq. (22). The black solid and dashed lines represents the regions where Eqs. (21) and (22) do not match the results of the simulations. 
The nucleation of small crystallites in front of the advancing liquid-crystal interface slows down the interface and results in lower apparent solidification front velocities in simulations performed with the EAM-FBD potential. A detailed discussion of the correlation between the homogeneous nucleation of crystallites inside the liquid phase and the velocity of the solidification front is provided section 4.4. In contrast with the nearly constant solidification front velocity, a gradual increase in the concentration of vacancies is detected in the simulations performed for $\mathrm{Ni}$, where the concentration is raising from $0.11 \times 10^{-2} \%$ to the maximum value $\sim 0.2 \%$. As explained for the EAM Cr system, at high temperature close to the melting point, the slow movement of the solidification front provides a sufficient time for the atoms to occupy the correct fcc lattice sites in the crystal structure and reduces the probability of vacancy formation. In the case of $\mathrm{Ni}$, there is no significant decrease in the solidification front velocity with increasing undercooling and the drop in the atomic mobility is contributing to the increase in the number of vacancies that are generated and trapped by the advancing solidification front. While the concentration of vacancies in EAM Ni system are still very high compared to the equilibrium value at the melting temperature, one vacancy per $10^{6}$ lattice sites, the comparison of the results obtained for the two metals show that the concentration of vacancies in bcc EAM Cr is twice higher as compared to fcc EAM Ni. As discussed earlier, this difference in the concentration is mostly related to the differences in the crystal structure between the two metals.

At this stage, the results that are obtained from the series of MD simulations for both metals can be compared to the ones obtained in the TTM-MD simulations of laser-induced generation of vacancies discussed in section 3.4. As illustrated in Fig. 4.4, for EAM Cr, the increase in the solidification front velocity from $103 \mathrm{~m} / \mathrm{s}$ to $139 \mathrm{~m} / \mathrm{s}$ in the range of temperatures from $0.95 T_{m}$ to $0.80 T_{m}$ results in the increase in the concentration of vacancies from $0.49 \%$ to $0.52 \%$. Meanwhile, for EAM Ni, the acceleration of the solidification front velocity from $52 \mathrm{~m} / \mathrm{s}$ to $171 \mathrm{~m} / \mathrm{s}$ leads to the increase in the concentration of vacancies from $0.011 \%$ to $0.044 \%$, Fig. 4.5. These values of vacancy concentrations are comparable to the ones obtained in TTM-MD simulations and illustrated in Fig. 3.3 and Fig. 3.4, where the surface temperature for most of the simulations covering the three regimes (melting and solidification, photomechanical spallation, and phase explosion regimes) ranges between the melting temperature and $0.80 T_{m}$ for both 
metals, and produce concentration of vacancies ranging between $0.49 \%$ and $0.52 \%$ for $\mathrm{Cr}$ and between $0.034 \%$ and $0.08 \%$ for $\mathrm{Ni}$.

The results for the velocity of solidification front obtained from MD simulations for the two metal systems are fitted to two analytical equations that have been suggested in literature for the description of the solidification kinetics $[205,206]$. In the high temperature region, where the thermodynamic driving force is very sensitive to temperature, the results of the solidification front velocity are fitted to Wilson-Frenkel expression:

$$
V(T)=C \exp \left(-Q / k_{B} T\right)\left[1-\exp \left(-\Delta G(T) / k_{B} T\right]\right.
$$

where $\Delta G$ is the difference in the free energies of the solid and liquid phases, $Q$ is the activation energy associated with the mobility of atoms in liquid phase, $k_{B}$ is the Boltzmann constant, and $C$ is a constant that depends on the interatomic spacing, the frequency for the atomic vibrations, and the average thermal velocity of atoms in liquid. In fitting this equation to the simulation results, $\Delta G$ is approximated as $\Delta G \approx L \Delta T / T_{m}$, where $L$ is the latent heat of melting and $\Delta T=T_{m}$ - $T$. The fitting of the equation to data points for $T_{m}>T>0.7 T_{m}$ yields the following parameters $C=14013 \pm 3815 \mathrm{~m} / \mathrm{s}$ and $Q=0.511 \pm 0.043 \mathrm{eV}$ for $\mathrm{Cr}$ and $C=6137 \pm 1726 \mathrm{~m} / \mathrm{s}$ and $Q=$ $0.231 \pm 0.027 \mathrm{eV}$ for $\mathrm{Ni}$.

In the low temperature region, at $T<T_{m}$ the velocity of the solidification front is dominated by the temperature dependence of the atomic mobility, which in Eq. (21) is represented by the term $\exp \left(-Q / k_{B} T\right)$. Accordingly, the results from MD simulations are fitted to a simple Arrhenius expression:

$$
V(T)=C_{1} \exp \left(-Q_{1} / k_{B} T\right)
$$

The fitting of this equation to the data points for $T<T_{m}$ gives $C_{1}=453 \pm 28.4 \mathrm{~m} / \mathrm{s}$, and $Q_{1}=$ $0.156 \pm 0.0072 \mathrm{eV}$ for $\mathrm{Cr}$ and $C_{1}=174 \pm 1.4 \mathrm{~m} / \mathrm{s}$ and $Q_{1}=0.32 \times 10^{-3} \pm 4 \times 10^{-5} \mathrm{eV}$ for Ni.

The combined application of the two equations provides a good description of the data points in Figs. 4.4 and 4.5, as shown by the solid and dashed red lines for the high and low temperature regions of the plots. 


\subsubsection{Atomic rearrangements near the liquid-solid interface}

To shed more light on the mechanisms responsible for the generation of vacancies at the rapidly advancing solidification front, an additional MD simulation is performed for EAM $\mathrm{Cr}$ following the same procedure as described in section 4.2 and the atomic rearrangement the solidification front are analyzed in detail. In these simulations, the system with the length of 100 $\mathrm{nm}$ is divided into 700 planes, each with thickness of one atomic plane and composed of 100 atoms. The atoms in this system are defined according to their identification number $\left(\mathrm{ID}_{\text {atom }}\right)$, the identification number of the atomic plane they belong to ( $\left.\mathrm{ID}_{\text {plane }}\right)$, the potential energy, and the coordinates of each atom.

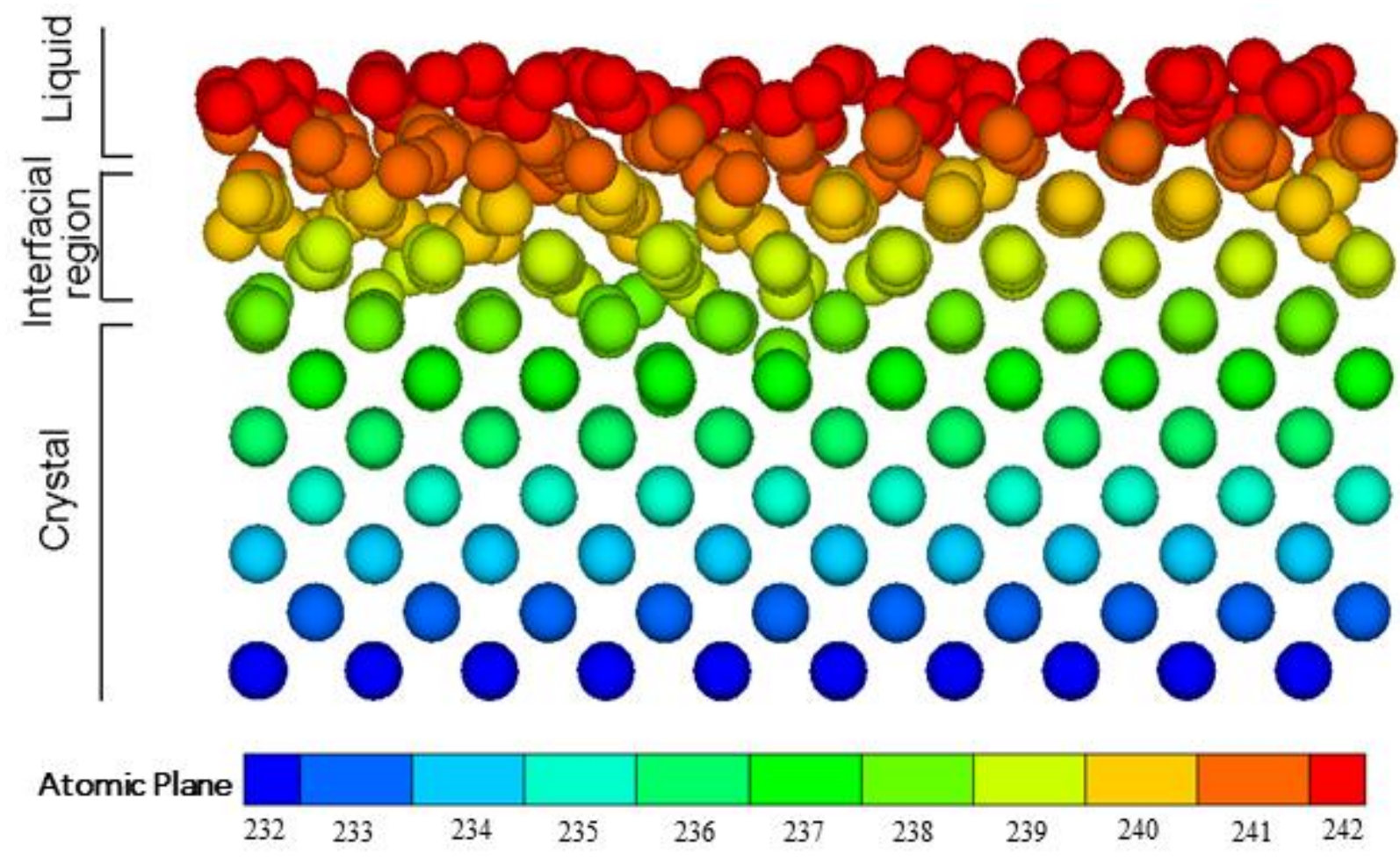

Fig. 4.6. Snapshot of an atomic configuration near the liquid-crystal interface obtained from MD simulation of solidification of the EAM Cr system at $0.50 T_{m}$. The snapshot is taken along [010] view direction and represents eleven atomic planes. The top upper plane is the liquid phase, the second two atomic planes are in the interfacial region and the following eight planes are the solid phase. The atomic configuration is quenched to $0 \mathrm{~K}$ in order to reduce the thermal noise in the atomic positions and energies. The atoms are colored according to the atomic planes they are associated with. The snapshot of the atomic configuration is taken at $102 \mathrm{ps}$ after quenching to $0.50 T_{m}$. 
Figure 4.6 shows a snapshot of atomic configuration near the liquid-crystal interface obtained in an MD simulation of EAM Cr performed at $0.50 T_{m}$. The atoms are colored according to the atomic planes they are associated with. The visual inspection of four atomic planes with identification numbers of 242 (liquid phase), 241 and 240 (interfacial region), and plane (crystal phase) at the time of 102 ps reveals the existence of 33 vacancies which are distributed as 6 vacancies in plane 239, 3 vacancies in plane 240, 14 vacancies in plane 241, and 10 vacancies in plane 242 (Fig. 4.7). Consequently, with the time progression, some of the atoms jumped from atomic planes with larger $\mathrm{ID}_{\text {plane }}$ to atomic planes with smaller $\mathrm{ID}_{\text {plane, }}$ in the direction of the solid phase. For example, at time 102 ps, plane 239 has 6 vacancies; and between time 102 ps and 104 ps three atoms from plane 239 are moved and annihilated vacancy sites in plane 238 (not shown in Fig. 4.7). During the same period of time (from 102 ps to 104 ps), 8 atoms from plane 240 jump to occupy vacancy sites in plane 239, which results in decreasing the number of vacancies in this plane from nine to only one vacancy at time $104 \mathrm{ps}$, and to zero vacancies at time 105.5 ps.

This analysis demonstrates that the annihilation of vacancy sites takes place due to the jumps of atoms from the nearest upper atomic plane. As represented in Fig. 4.7, we labeled the atoms that are going to move to their new position in an adjacent atomic plane by a cross ribbons colored by the color of their new atomic plane. Meanwhile, we encircle the atoms that have jumped to a new plane with a ribbon colored according to the color associated with their initial atomic plane. For instance, at time 102 ps, some atoms in plane 240 are surrounded by green cross ribbons to give an indication that these atoms are going to move and annihilate vacancies in plane 239 at time 104 ps. Simultaneously, at time 104 ps, the same atoms that had green cross ribbons in plane 240 at 102 ps are now marked by a yellow ribbon in plane 239 to denote their new position. On a continuing basis, the process of formation and annihilating of vacancies is monitored for longer than $12 \mathrm{ps}$ in order to observe the transformation of plane 242 from liquid to solid phase, and to identify any vacancy stabilization in the solid phase. Ultimately, in spite of the fact that the liquid-solid interface has progressed to plane 248 , and moved $\sim 0.9 \mathrm{~nm}$ from plane 242, one stable vacancy is left behind in plane 241. 


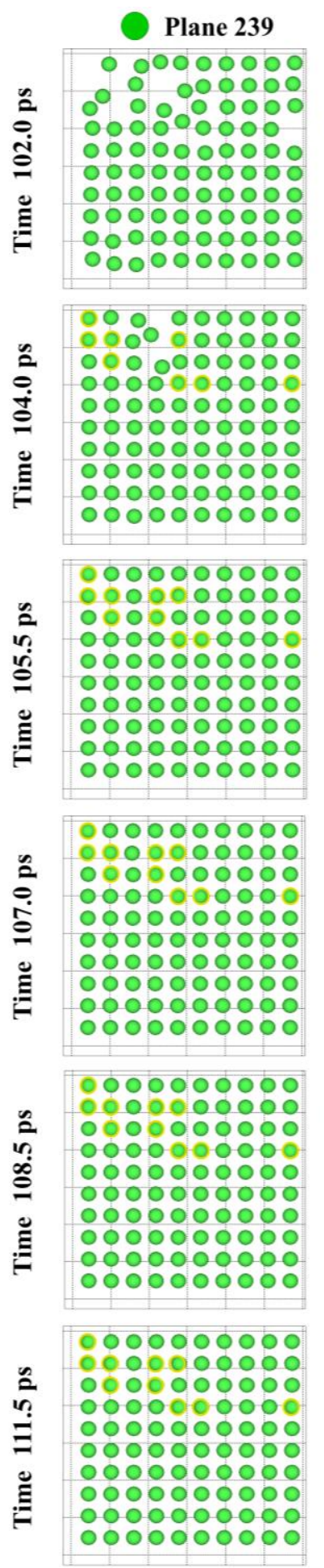

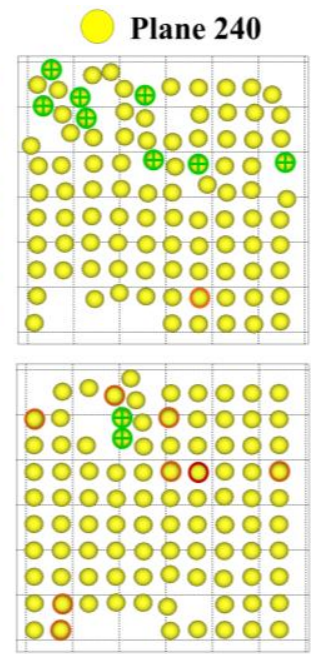

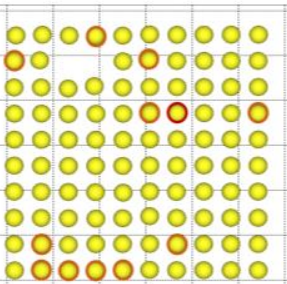

0000000000

\section{0 \\ 000000000 \\ 0000000000 \\ 0000000000 \\ 0000000000 \\ 0000000000 \\ 0000000000 \\ 0000000000 \\ 0000000000 \\ 0000000000}

\section{0}

0000000000

0000000000

0000000000

0000000000

0000000000

0000000000

0 000000000

0000000000

0000000000

\section{0 \\ 0000000000 \\ 0000000000 \\ 0000000000 \\ 0000000000 \\ 0000000000 \\ 0000000000 \\ 0000000000 \\ 0000000000}

0000000000
Plane 241

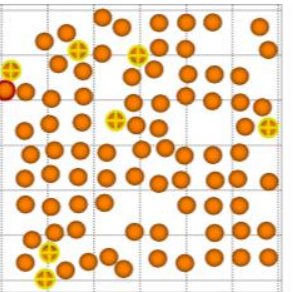

0.0000000

0000000 000000000 0000000000 - o o o ० O००

0000000000 0000000 00000000 000000000 - $90^{\circ} 0^{\circ} 000$

0000000000 0000 000000000 0000000000 ००00000000 0000000000 000000000 10000000 000000000 0000000000

0000000000 000000000 0000000 0000000000 0.0000000 0000000000 0000000000 0000000000 0000000 0000000000

0000000000 0000000000 0000000000 0000000000 0000000000 0000000000 0000000000 0000000000 100 ००००००००००

0000000000 0000000000 0000000000 0000000000 - 0000000०० 0000000000 0000000000 0000000000 000000000 ०००००००००
Plane 242
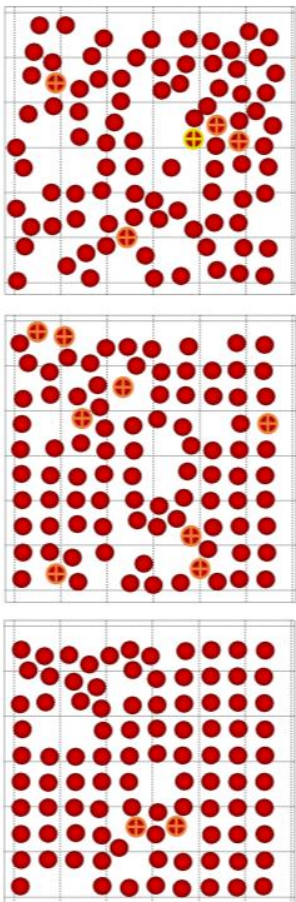

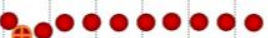

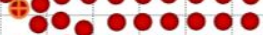

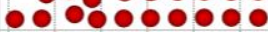

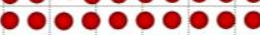

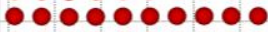

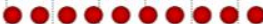
$\bullet \bullet \bullet \bullet \bullet \bullet \bullet \bullet \bullet$ $\bullet \bullet \bullet \bullet \bullet \bullet \bullet \bullet \bullet$ $\bullet \bullet \bullet \bullet \bullet \bullet \bullet \bullet \bullet \bullet ~$

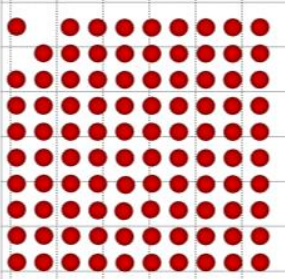

(1)

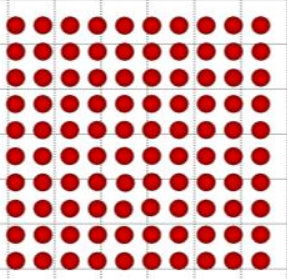

Fig. 4.7. Close up view of four atomic planes that, at the initial time of 102 ps include liquid phase plane (plane 242), two interfacial planes (planes 241 and 240), and solid phase plane (plane 239). The atoms are colored according to the atomic planes they are associated with. The atoms with cross ribbons represent the atoms that are going to move to the new atomic plane at a later time and the color of the cross ribbons represent the color of their new atomic plane. The atoms surrounded with ribbons represent the atoms that changed their positions to a new atomic plane, and the color of the ribbons correspond to the color of their initial atomic plane. 
Additionally, our investigation shows that some of the atoms can jump back from the lower to the upper atomic planes and occupy a position close to the vacancy sites they left behind, and stay there from 0.2 up to 2 ps before they move back to their initial site and annihilate the corresponding vacancy site. This probably is related to the interstitial dumbbell configuration where atom can jump and occupy a position along $\langle 110\rangle$ direction. One can infer several points from the analysis provided in this section. First, as was mentioned earlier, the transition of atoms from the liquid to the solid phase results in the advancement of the liquidsolid interface. The detailed investigation of the atomic rearrangements near the interface region confirms this mechanism and shows that the direction of the atomic flow is opposite to the direction of the vacancies flow. Second, the dominant mechanism responsible on the vacancy formation is found to be related to the rapid movement of the solidification front during the solidification process. The number of vacancies generated at the advancing solidification front is defined by the ability of the atoms to migrate between the planes and to fill the vacant sites in the new planes generated in the solidification process. The temperature controls the rate of the thermally-activated atomic rearrangements at the interface, while the velocity of the interface defines the time available for the rearrangements.

In order to further support the conclusion on the key role of the rapid advancement of the solidification front in the generation of vacancies, an MD simulation at a temperature of $0.98 T_{m}$ is performed for a fully crystalline EAM $\mathrm{Cr}$ system with the same computational size as described in section 4.2. The analysis of the simulation results indicates that not a single vacancy is generated through the mechanism of thermally activated generation of Frenkel pairs during the simulation time of $100 \mathrm{ps}$.

\subsubsection{Vacancy diffusion coefficient and time between the vacancy jumps}

To evaluate the kinetics of vacancies diffusion in the EAM Cr and the EAM Ni systems, the diffusivity of vacancies is calculated. To calculate the diffusion coefficient of a vacancy, $D_{v}$, a series of MD simulations for bcc and fcc crystal structures each contain a single vacancy are performed at different temperatures ranging between $0.95 T_{m}$ and $0.10 T_{m}$. At each specific temperature, the diffusion coefficient is calculated according to the Einstein relation, $D_{v}=\frac{1}{6 t}\left\langle\left|\vec{R}_{i}\left(t_{0}+t\right)-\vec{R}_{i}\left(t_{0}\right)\right|^{2}\right\rangle_{i, t_{0}}$, where $\vec{R}_{i}(t)$ is the vacancy's position at time $t$ in the $i^{t h}$ 
simulation. In order to improve the statistical accuracy of the calculation, an average over the starting times $t_{0}$ along the MD trajectories is considered. The values of the diffusion coefficient obtained for different temperatures are fitted to the Arrhenius dependence, $D_{v}(T)=D_{v}^{0} \exp \left(\frac{-E_{v}^{m}}{k_{B} T}\right)$, yielding the vacancy migration energy $E_{v}^{m}=0.81 \mathrm{eV}$, and preexponential factor $D_{v}^{0}=0.39 \times 10^{-2} \mathrm{~cm}^{2} / \mathrm{s}$ for the EAM Cr, Ref. [129], while for the EAM Ni, $E_{v}^{m}=1.49 \mathrm{eV}$, and $D_{v}^{0}=0.137 \times 10^{-2} \mathrm{~cm}^{2} / \mathrm{s}$. The knowledge of the vacancy diffusion coefficient allows us to compute the time between the vacancy jumps at each temperature, through the calculation of the vacancy jump rate, $\frac{1}{\tau}=6 D_{v} / r_{1}^{2}$, where $r_{1}$ is the nearest neighbor distance equal to $r_{1}=\sqrt{3 / 4} a$ in bcc lattice structure and $r_{1}=\frac{a}{2 \sqrt{2}}$ in fcc lattice structure, and $a$ represents the lattice parameter.

The results of the calculations of the vacancy diffusion coefficient and the time between jumps are plotted as a function of the normalized temperature in Fig. 4.8. Although the overall picture for both systems is similar at the first sight, the results illustrate differences in the magnitude of the diffusivity between $\mathrm{Cr}$ and $\mathrm{Ni}$. For the EAM $\mathrm{Cr}$, at a high temperature close to $T_{m}$, the diffusivity reaches a maximum value of $\sim 0.69 \times 10^{-4} \mathrm{~cm}^{2} / \mathrm{s}$, while the time between jumps is at its minimum value $\sim 1.5 \times 10^{-3} \mathrm{~ns}$. As the temperature decreases, the diffusivity drops and the time between jumps increases by many orders of magnitude, e.g., $D_{v} \approx 1.4 \times 10^{-20} \mathrm{~cm}^{2} / \mathrm{s}$ and $\tau \approx$ $7.4 \times 10^{12} \mathrm{~ns}=7400 \mathrm{~s} \approx 2$ hours $0.10 T_{m}$. These results are consistent with the ones obtained in TTM-MD simulation for a Cr target irradiated by 200 fs laser pulse [129], where the diffusivity of $\sim 10^{-7} \mathrm{~cm}^{2} / \mathrm{s}$ and the time between jumps of $27 \mathrm{ps}$ are estimated for the surface temperature of $1180 \mathrm{~K}$. In contrast to EAM Cr, the maximum value of the diffusivity at $T_{m}$ is much lower in EAM Ni, $D_{v}\left(T_{m}\right) \approx 8.6 \times 10^{-9} \mathrm{~cm}^{2} / \mathrm{s}$, which corresponds to $\tau \approx 3 \mathrm{~ns}$. These values change to $D_{v} \approx$ $1.6 \times 10^{-38} \mathrm{~cm}^{2} / \mathrm{s}$ and $\tau \approx 1.7 \times 10^{30} \mathrm{~ns}$ at $0.15 T_{m}$. This big difference in the diffusivity between the two systems is related to the difference in the crystal structure between the close-packed fcc $\mathrm{Ni}$ and a more open bcc structure of $\mathrm{Cr}$. 

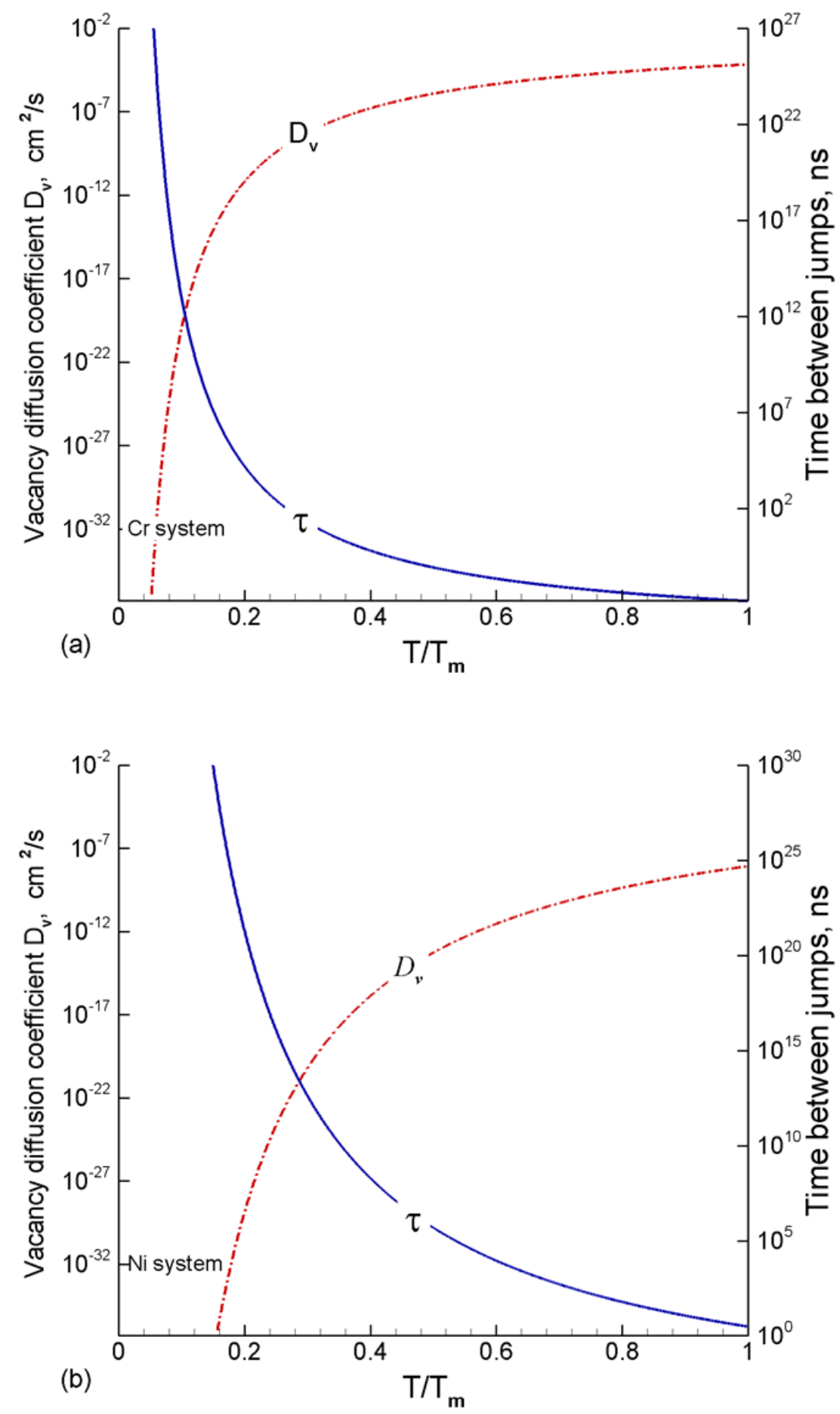

Fig. 4.8. The vacancy diffusion coefficient, $D_{v}$, and the time between jumps, $\tau$, predicted in MD simulations for (a) EAM Cr, and (b) EAM Ni systems. The temperature is normalized by the melting temperatures for both systems. 
The differences in the diffusivity between the two metals is also supported by the available experimental data indicating that the mobility of the vacancies in bcc metals is much higher than that in fcc metals at the same normalized temperature $T / T_{m}$. In particular, the ratio of the monovacancy migration to the formation enthalpy, $H_{1 V}^{M} / H_{1 V}^{F}$ is between 0.1 and 0.5 for bcc metals and between 0.75 and 0.95 for fcc metals [207], confirming that the mobility of vacancies is substantially higher in bcc metals as compared to fcc metals.

\subsection{The effect of homogeneous nucleation in the liquid phase on the solidification front velocity}

The dependence of the solidification front velocity on temperature for EAM Ni, which is discussed in section 4.3.2, exhibits substantial difference from the one for Cr, especially at low temperatures. As illustrated in Fig. 4.5, at temperature close to the melting temperature, the solidification front velocity shows an approximately linear dependence on temperature down to $0.8 T_{m}$, while at lower temperatures this behavior changes and the velocity remains nearly constant with the continued reduction of temperature down to $0.1 T_{m}$. On the other hand, Ashkenazy and Averback presented slightly different results for the temperature dependence of the solidification front velocity for $\mathrm{Ni}$ [208], where the interatomic interaction is described by the EAM potential in the form suggested by Foiles, Baskes, and Daw (FBD) [128]. According to their results, at high temperatures, the solidification front velocity in Ni exhibits the same trend that has been observed in our results, i.e., the linear dependence on the temperature down to $\sim 0.8 T_{m}$. The difference between the results emerges at low temperature, where the solidification front velocity in Ref. [208] decelerates with the continued reduction of temperature.

To elucidate the reasons for the divergence between the results obtained for $\mathrm{Ni}$ with the EAM-Johnson and EAM-FBD potentials, another series of MD simulations is performed for Ni using the same computational approach and the same size of the computational system that is described in section 4.2. In these simulations, the interatomic interaction in $\mathrm{Ni}$ is described by EAM-FBD potential [128], and a cut-off function is added to this potential in order to smoothly bring the interaction energies and forces to zero at interatomic distance of $5.5 \AA$ [209]. The EAM-FBD potential is fitted to low-temperature values of the equilibrium lattice constant, sublimation energy, bulk modulus, elastic constants, and vacancy formation energy. The 
equilibrium melting temperature, $T_{m}$, of pure EAM-FBD $\mathrm{Ni}$ is calculated in a series of liquidsolid coexistence MD simulations at zero pressure, with the same method that is used to determine the melting temperature for $\mathrm{Ni}$ with the EAM-Johnson potential. The results of the calculations yield that $1719 \mathrm{~K}$ as the equilibrium melting temperature for EAM-FBD Ni, which is about $0.3 \%$ below the experimental value of the melting temperature of $\mathrm{Ni}, 1725 \mathrm{~K}[210]$.

In these simulations, the computational cell is divided into 30 layers with a thickness of each layer equal to $1.8 \mathrm{~nm}$. The average local temperature of each layer is controlled independently in order to avoid the local temperature increase at the rapidly advancing interface due to the release of the heat of melting. In total, 52 simulations of the EAM-FBD Ni system are performed at different undercooling temperatures and constant zero pressure. The temperature variation is from $0.95 T_{m}$ to $0.1 T_{m}$ with a temperature increment equal to 0.02 . In order to define the error bars which represent the standard deviation of the solidification front velocity, three sets of the 52 simulations are performed, each starting with different initial conditions.

\subsubsection{The differences in the solidification front velocity in $E A M-F B D \mathrm{Ni}$ and EAM-Johnson Ni}

The procedure that is used in this section for the purpose of determining the velocity of solidification front is similar to the ones used to define the velocity of solidification front for EAM Cr and EAM-Johnson Ni. Initially, the system is thermalized at a temperature close to the melting temperature for $200 \mathrm{ps}$. Then the temperature of three quarters of the system is increased to above the melting temperature by scaling the velocity of each atom, so that liquid phase is created in this region while the rest of the system remains at a temperature slightly below $T_{m}$. Following this step, the liquid-solid coexistence system is relaxed at the equilibrium melting temperature before quenched to different target temperatures. In order to avoid any artifacts due to the rapid change in volume of the system quenched from the melting to the target temperature, we exclude the first 10 ps from our calculation for the solidification front velocity. 

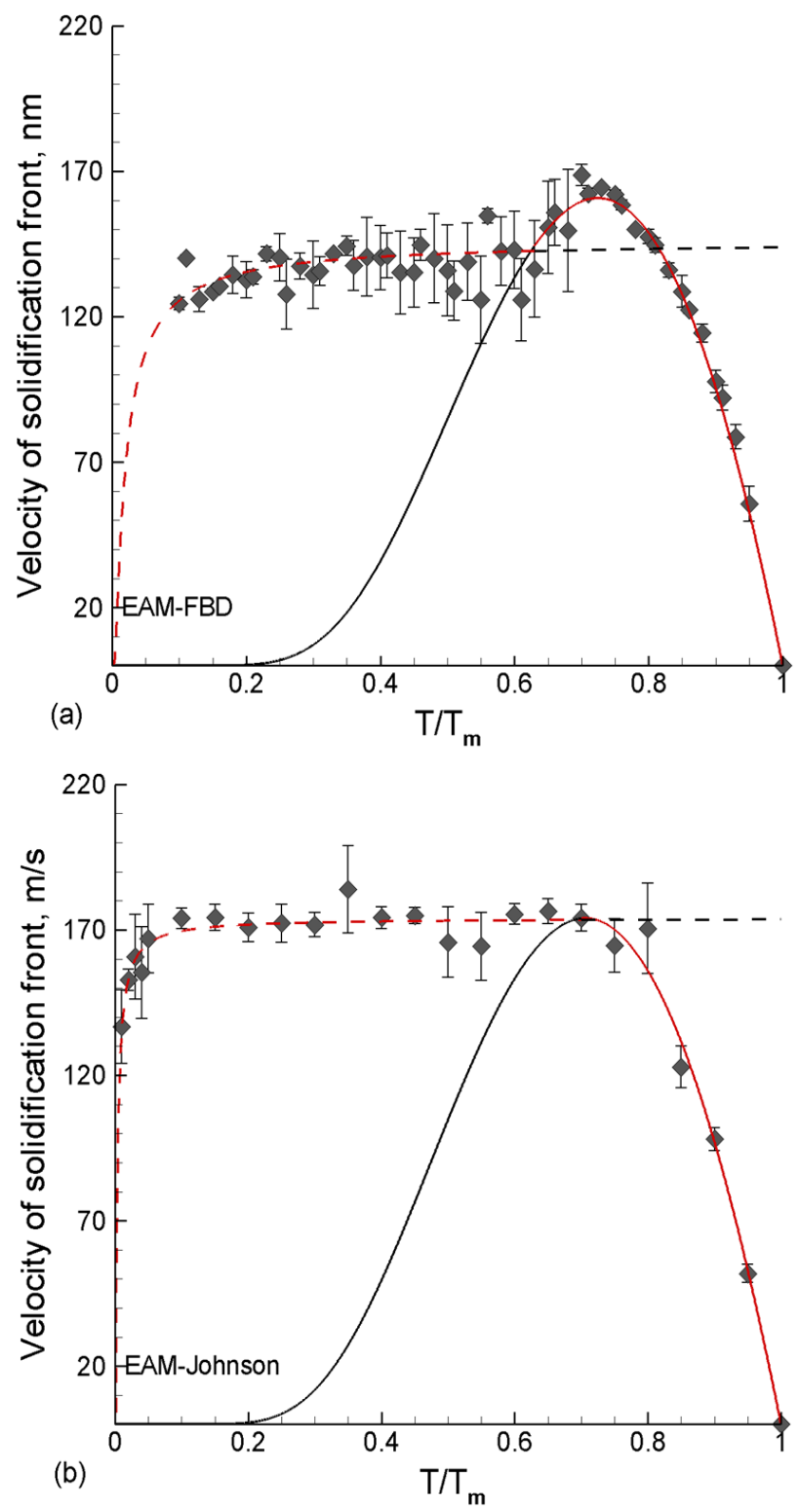

Fig. 4.9. Solidification front velocity as a function of normalized temperature for Ni system where the interatomic interaction between $\mathrm{Ni}$ atoms is described by the EAM-FBD [128] (a) and EAM-Johnson [182] (b) potentials. The error bars in both (a) and (b) represent the standard deviations of the solidification front velocity predicted in three sets of MD simulations, each starting with different initial conditions. The red solid lines represent the fitting of the MD results to Eq. (21), while the dashed red lines represent the fitting of the MD results to Eq. (22). The black solid and dashed lines correspond to regions where Eqs. (21) and (22) cannot provide an adequate description of the computational results. 
As described earlier, at melting temperature the free energy of the solid and liquid phases are equal and the system is in the liquid-crystal coexistence state. As the system goes through undercooling process, the free energy of the two phases is changing and the difference in the free energy between the two phases creates the driving force for the solidification process. Accordingly, the average velocities of solidification front obtained from the series of MD simulations of EAM-FBD $\mathrm{Ni}$ are plotted in Fig. 4.9(a) with respect to the temperature normalized by the melting temperature of the model material.

The curve of the velocities in Fig. 4.9(a) shows similarity with the one presented by Ashkenazy and Averback [208] and, at the same time, exhibits both similarities and differences from the one obtained for EAM-Johnson Ni. In both cases, for EAM-FBD Ni and EAM-Johnson $\mathrm{Ni}$, one can define the high and low temperature regions in the curves of the solidification front velocities. In the high temperature region, in the temperature range extending from the melting temperature down to $0.7 T_{m}$, the velocity of solidification front increases approximately linearly from zero up to the maximum value of slightly above $170 \mathrm{~m} / \mathrm{s}$. The differences in the velocity curves start to appear below $0.7 T_{m}$. In particular, in EAM-FBD Ni, the velocity decreases from $\sim 174$ to $125 \mathrm{~m} / \mathrm{s}$ as the temperature reduces from 0.7 to $0.6 T_{m}$ and continues to fluctuate between 154 to $124 \mathrm{~m} / \mathrm{s}$ as the temperature is further reduced down $0.1 T_{m}$. This behavior in the velocity in the low temperature region is different from what is observed for EAM-Johnson Ni, where the velocity fluctuates around the maximum value, $\sim 174 \mathrm{~m} / \mathrm{s}$, in a broad range of temperature, from 0.7 to $0.1 T_{m}$, Fig. 4.9(b). The results obtained from this series of MD simulations are also fitted to the analytical equations discussed above, Eqs.(21) and (22), and the fitting parameters for EAM-FBD Ni are $C=7657 \pm 643 \mathrm{~m} / \mathrm{s} Q=0.307 \pm 0.01 \mathrm{eV}$ in the high temperature region, where Eq. (21) is used for data points obtained for $T \geq 0.7 T_{m}$, and $C_{1}=146 \pm 2.67 \mathrm{~m} / \mathrm{s} Q_{1}=0.224 \times 10^{-}$ ${ }^{2} \pm 0.72 \times 10^{-3} \mathrm{eV}$ in the low temperature region, where Eq. (22) is fitted to the data points calculated for $T<0.7 T_{m}$. The results of the fitting are shown in Fig. 4.9(b). 

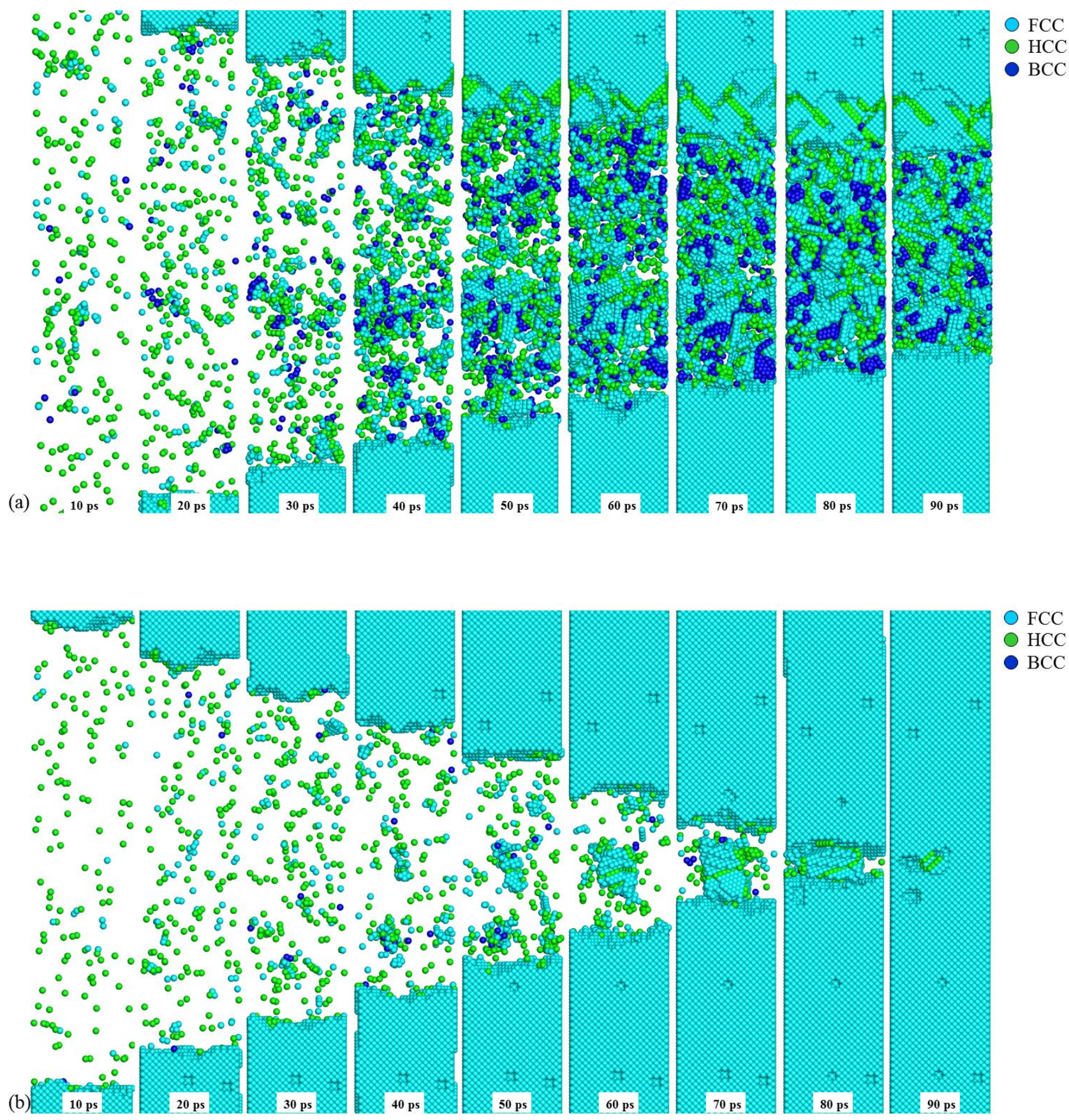

Fig. 4.10. Snapshots of the atomic configurations obtained in MD simulations of solidification at $0.6 T_{m}$ in a Ni system described by EAM-FBD (a) and EAM-Johnson interatomic potentials. The snapshots are taken along the [010] view direction. The atomic configurations are quenched to $0 \mathrm{~K}$ for $1 \mathrm{ps}$ to reduce thermal noise in atomic positions and energies. The atoms are colored according to their local structure environment, with the light blue, dark blue and green colors corresponding to the fcc, bcc, and hcp structures, respectively. All the atoms that belong to the liquid phase and have unknown structure environment are blanked. 
In order to provide a clear understanding of the origin of the differences in the solidification front velocities predicted for $\mathrm{Ni}$ with the two interatomic potentials, several snapshots of the atomic configurations obtained in simulations performed at $0.6 T_{m}$, are shown in Fig. 4.10. The atoms in these snapshots are colored according to their local structure environment [211], where the light blue, dark blue and green atoms belong to fcc, bcc, and hcp crystal structures, respectively, while, all atoms that belong to the liquid phase and have unknown local structure environment are blanked. The analysis of the atomic configurations in the EAM-FBD Ni system reveals the formation of some crystalline structures inside the liquid phase just $10 \mathrm{ps}$ after quenching to $0.6 T_{m}$. The nucleation of solid structures inside the liquid phase is called "homogeneous nucleation" and can be described by the classical nucleation theory (CNT) [212, 213, 214], where the Gibbs free energy changes upon the formation of the solid nucleus of radius $r$ in an undercooling liquid is described as

$$
\Delta G_{r}=-\frac{4}{3} \pi r^{3} \Delta G_{v}+4 \pi r^{2} \gamma_{S L}
$$

where $\Delta G_{v}$ is the Gibss free energy difference between the solid and liquid phases per unit volume and $\gamma_{S L}$, is the liquid-solid interficial energy. The general relationship between the critical size of the solid nucleus and the undercooling tempertaure can be obtained by differntiation of Eq. (23):

$$
r^{*}=\frac{2 \gamma_{S L}}{\Delta G_{v}}=\left(\frac{2 \gamma_{S L} T_{m}}{L_{v}}\right) \frac{1}{\Delta T} .
$$

Following the relationship in Eq. (24), if a solid cluster is formed inside the liquid with radius smaller than $r^{*}$, this cluster is considered to be unstable and it decays spontaneously in order to lower the free energy of the system. The clusters with radii larger than $r^{*}$, on the other hand, will grow in order to decrease the free energy of the system. Eq. (24) can also be reformulated in order to obtain the temperature of unstable equilibrium for a cluster of a given radius $r$ :

$$
T^{*}=T_{m}\left[1-\frac{2 \gamma_{S L}}{L} \frac{1}{r}\right] .
$$


According to this equation, if the temperature of the liquid phase, $T_{L}$, is larger than $T^{*}$, the system decreases its free energy by gradually dissolving the solid cluster, but if $T_{L}$ is less than $T^{*}$ the cluster continues to growth.

In order to relate the discussion of CNT to our results, the fraction of the crystalline structures that are formed inside the liquid phase has been determined. The results show that as the total molar fraction of atoms in the solid clusters reaches $\sim 3 \%$ the cluster continue to grow inside the undercooled liquid, whereas below this fraction the clusters slowly dissolve. As it is shown in Fig. 4.10 (a) for EAM-FBD Ni, the first solid clusters appear in the upper and middle parts of the liquid region as early as $10 \mathrm{ps}$ after quenching to $0.6 T_{m}$. Some of the small clusters are captured by the advancing liquid-solid interface, while yet others continue to grow in the undercooled liquid.

Turning to the implications of the homogeneous nucleation of new crystallites on the solidification front velocity, the snapshots illustrate that the fast growth of the crystallites gradually impedes the movement of the primary liquid-solid interface and results in deceleration of the velocity of the solidification front from 180 down to $\sim 126 \mathrm{~m} / \mathrm{s}$ during $30 \mathrm{ps}$. These observations can be compared to the results obtained for EAM-Johnson $\mathrm{Ni}$, where the same structural analysis reveals a much less active homogeneous nucleation and growth of new crystallites, Fig. 4.10(b). The first crystalline structures are formed at around $30 \mathrm{ps}$ and continue to growth until they are incorporated into the advancing solidification front. The formation of the small solid clusters in this case does not strongly affect the advancement of the liquid-solid interface and the velocity of solidification front stays at almost the same value, $\sim 174 \mathrm{~m} / \mathrm{s}$, until the complete solidification of the system.

To further investigate the effect of the homogeneous nucleation on the solidification front velocity, the structural analysis of atomic configurations is extended to $0.3 T_{m}$, which is below the glass transition temperature of $\mathrm{Ni}$ system described by both potentials. The results of the analysis are shown in Fig. 4.11. Although the overall picture of the nucleation of small crystallites in the supercooled liquid is similar to the one discussed above for $0.6 T_{m}$, the lower atomic mobility at $0.3 T_{m}$ is reflected in a much slower growth of the new crystallites. For example, while first crystallites can be identified in the EAM-FBD Ni as early as 15 ps, Fig. 4.11(a), the total fraction of the crystalline atoms inside the undercooled liquid grows slowly from $\sim 2 \%$ at 15 ps to $\sim 11.4 \%$ at 120 ps just before the two interfaces meet each other. 


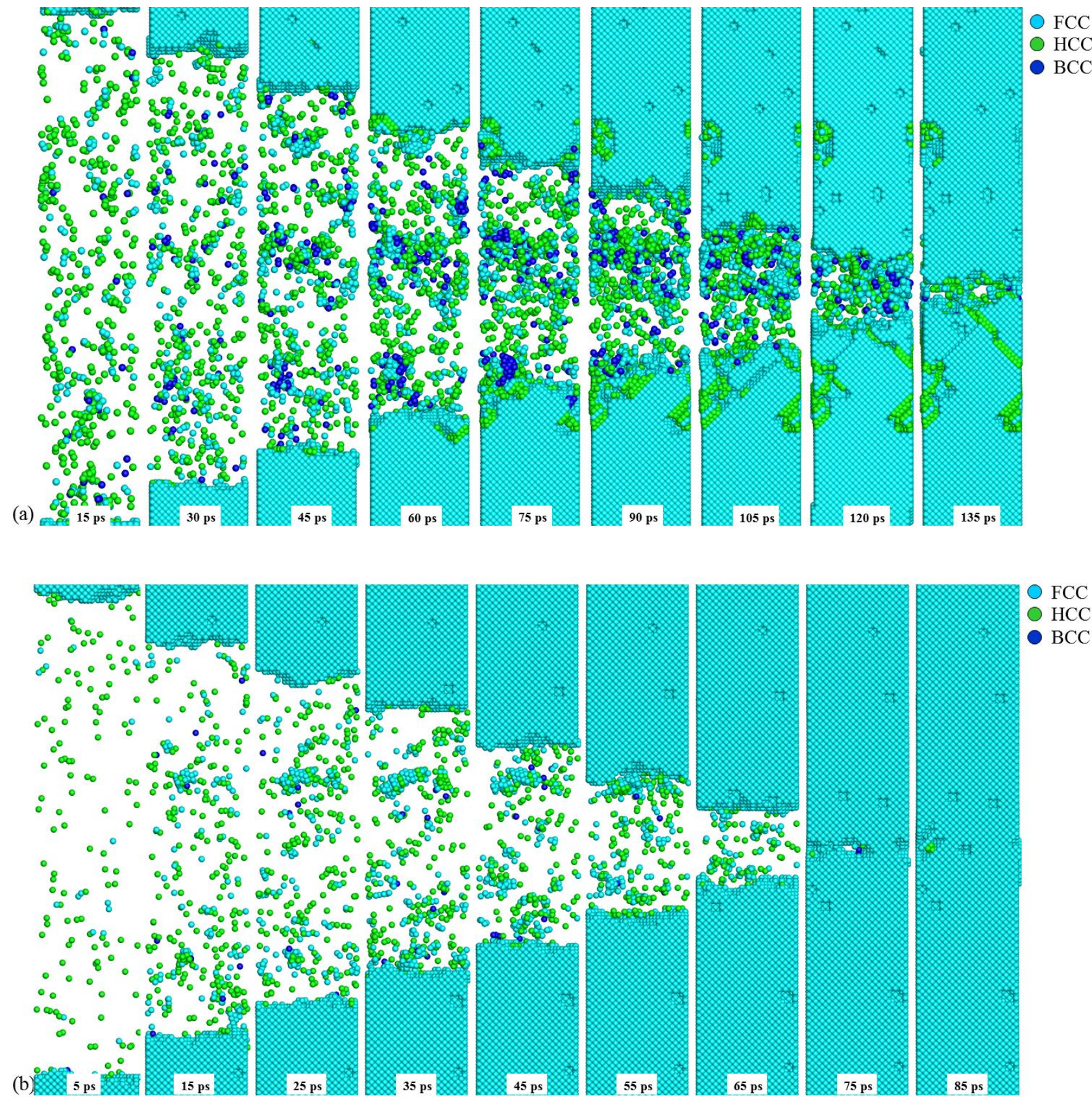

Fig. 4.11. Snapshots of the atomic configurations obtained in MD simulations of solidification at $0.3 T_{m}$ in a Ni system described by EAM-FBD (a) and EAM-Johnson interatomic potentials. The snapshots are taken along the [010] view direction. The configurations are quenched for 1 ps to reduce thermal noise in atomic positions and energies. The atoms are colored according to their local structure environment, with the light blue, dark blue and green colors corresponding to the fcc, bcc, and hcp structures, respectively. All the atoms that belong to the liquid phase and have unknown structure environment are blanked. 
In contrast, at $0.6 T_{m}$, the fraction of the crystalline atoms in the liquid phase growth rapidly from $\sim 2 \%$ at 10 ps to $\sim 30 \%$ at 90 ps. Accordingly, the formation of the solid structures inside the liquid phase results in a smaller deceleration of the solidification front at this stronger undercooling, as discussed in more detail below.

In the simulation performed at $0.3 T_{m}$ with EAM-Johnson potential, the density of the crystalline atoms is much smaller. In Fig. 4.11(b), we can see the formation of a small solid structure inside the liquid phase at $15 \mathrm{ps}$. This cluster slowly grows in size until it merges with one of the advancing liquid-solid interface at $\sim 55 \mathrm{ps}$. Similarly to the results discussed above for $0.6 T_{m}$, the velocity of solidification front at $0.3 T_{m}$ remain roughly constant during the solidification process, at the level of $\sim 170 \mathrm{~m} / \mathrm{s}$, and is apparently unaffected by the homogeneous nucleation of solid clusters.

At this stage we realized the influence of the homogeneous nucleation on the velocity of solidification front and, consequently, extended the analysis of the nucleation kinetics to all simulations performed for EAM-FBD Ni and EAM-Johnson Ni. Fig. 4.12 represents the fraction of crystalline regions in the liquid phase during solidification process for six MD simulations performed at target temperatures of $0.9,0.8,0.7,0.6,0.5$, and $0.3 T_{m}$ for (a) EAM-FBD and (b) EAM-Johnson potentials. For EAM-FBD Ni, Fig. 4.12(a), at 0.9, 0.8, and $0.7 T_{m}$ the fraction of the crystalline region in the liquid phase remains within almost the same value, $0.67 \%$, during the first $90 \mathrm{ps}$. The low fraction of crystalline regions in these three simulations indicates that although small crystalline embryos can form spontaneously in the undercooled liquid, the radii of these embryos remain below the critical radius and the system lower its free energy by dissolving these embryos. The remarkable increase in the fraction of the crystalline regions is observed in the simulation with $0.6 T_{m}$, where it immediately increases up to $\sim 1.8 \%$ just after 10 ps and continue to growth in a fast manner until the fraction reaches $\sim 40 \%$ after 155 ps. 

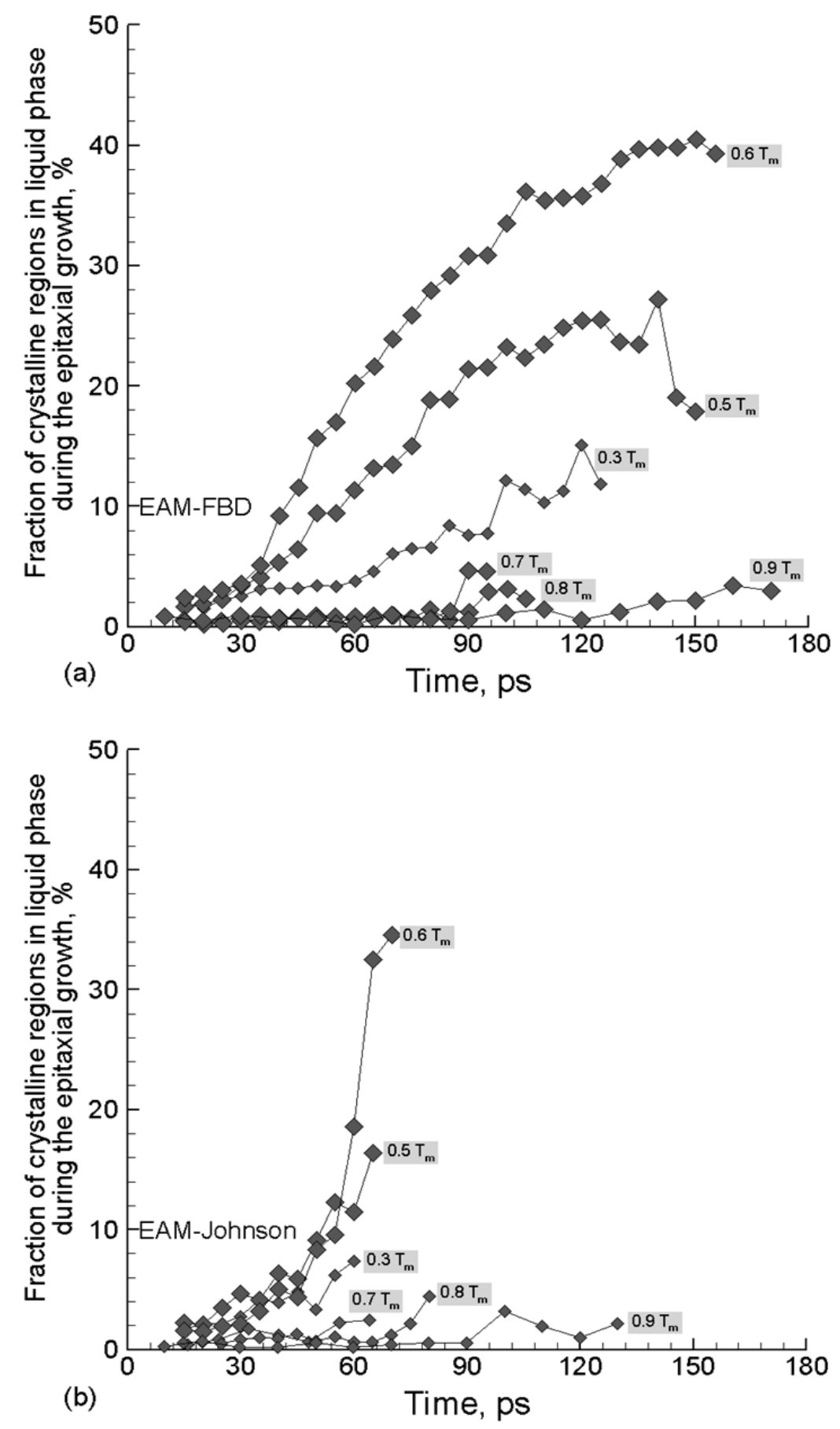

Fig. 4.12. The evolution of the fraction of crystalline regions in the liquid phase predicted in MD simulations of solidification of Ni systems described by (a) EAM-FBD and (b) EAM-Johnson potentials. Initially, the two liquid-solid Ni systems are well equilibrated at melting temperature and then quenched to different desired temperatures. The results are shown for six target temperatures: $0.9,0.8,0.7,0.6,0.5$, and $0.3 T_{m}$. 
This simulation is technically stopped at 160 ps after the solidification time due to the sluggish movement of the liquid-solid interface that is impeded by the solid volume which is formed and grew inside the liquid. With further decrease of the solidification temperature, the atomic mobility and the rate of growth of the crystalline clusters decrease, leading to the corresponding drop in the maximum fraction of the crystalline regions to $25 \%$ and $15 \%$ for 0.5 and $0.3 T_{m}$, respectively. In line with the above results, one can consider $0.6 T_{m}$ is the vertex of the Time Temperature Transformation (TTT) diagram for pure Ni system.

In the case of EAM-Johnson $\mathrm{Ni}$, at high temperature simulations, $0.9,0.8$, and $0.7 T_{m}$, the fraction of crystalline regions remains below $0.6 \%$ during the first 50 ps after the quenching, which is similar to what is observed in the EAM-FBD $\mathrm{Ni}$ simulations at these target temperatures. The fraction of the crystalline regions increases more rapidly at $0.6 T_{m}$ and approaches $\sim 9 \%$ at $50 \mathrm{ps}$. The sharp increase in the fraction of crystalline regions during the last $15 \mathrm{ps}$ of the simulation is related to the nucleation and growth of an individual cluster inside the liquid phase, which eventually comes in contact with the two solidification fronts at $\sim 70$ ps as shown in Fig. 4.10(b). The contrast between the relatively slow growth of this cluster and the sharp increase in the fraction of the crystalline regions during the last 20 ps of the simulation can be explained by the fast reduction of the liquid part of the system due to the propagation of the solidification fronts. The values of the fraction of the crystalline structures remain within the same level, $\sim 9 \%$ and $4 \%$ for 0.5 and $0.3 T_{m}$, as the temperature is further reduced, Fig. 4.12(b).

The interconnection between the homogeneous nucleation and the velocity of the solidification front is also investigated in more detail by measuring the fraction of liquid volume (FLV) which is directly related to the advancement of the liquid-solid interface. The FLV has been measured in all simulations performed with the EAM-FBD and EAM-Johnson potentials and the results are plotted with respect to the solidification time for the six of the simulations in Fig. 4.13. The comparison between the results from the two series of MD simulations indicate that: 

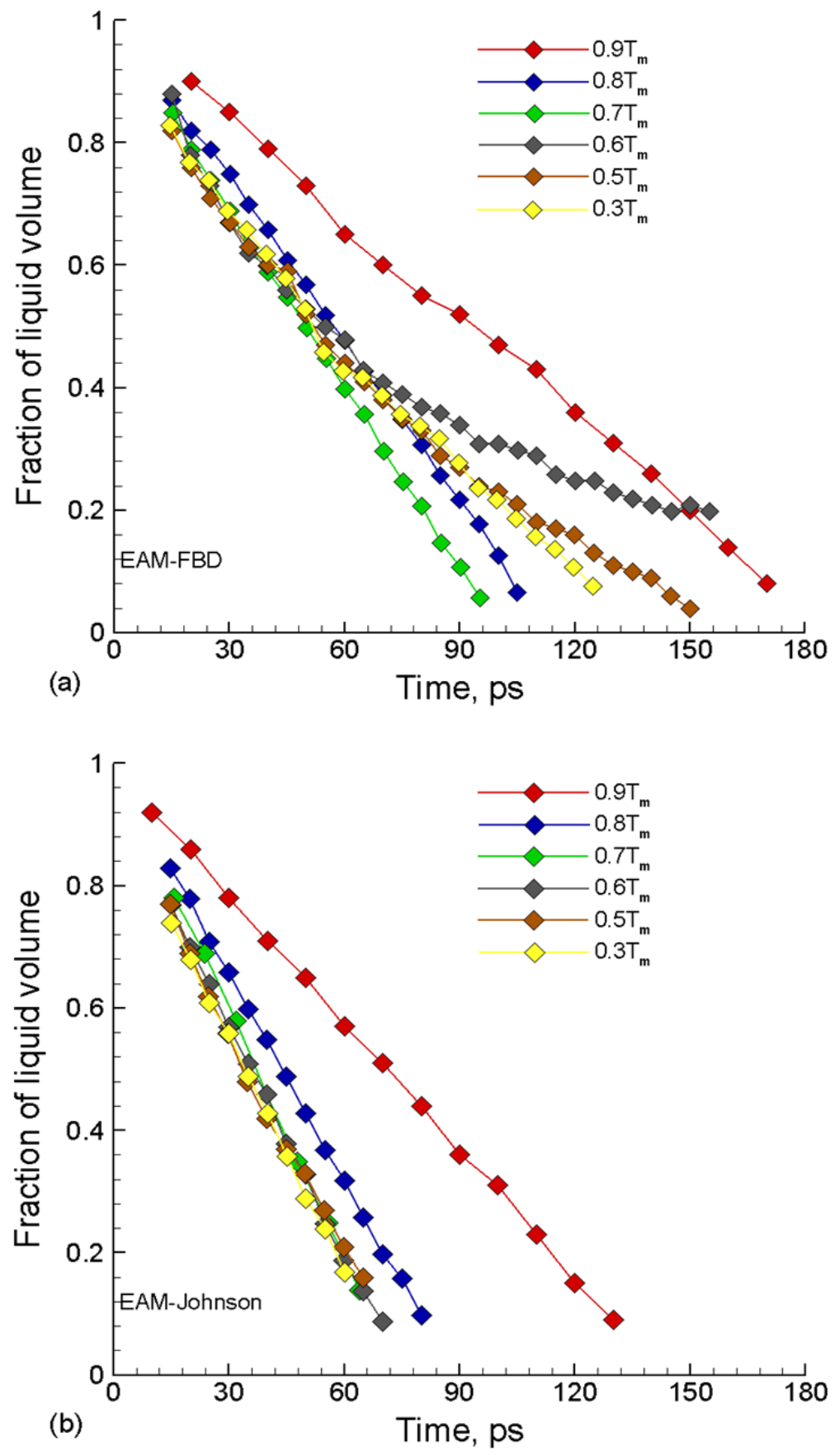

Fig. 4.13. The evolution of the fraction of liquid volume predicted in MD simulations of solidification of $\mathrm{Ni}$ systems described by (a) EAM-FBD and (b) EAM-Johnson potentials. Initially, the two liquid-solid Ni systems are well equilibrated at melting temperature and then quenched to different desired temperatures. The results are shown for six target temperatures: $0.9,0.8,0.7,0.6,0.5$, and $0.3 T_{m}$. 
(1) At a high temperature of $0.9 T_{m}$, and due to the relatively low driving force for the phase transformation, the solidification process takes longest time to complete, 174 ps and 134 ps for EAM-FBD $\mathrm{Ni}$ and EAM-Johnson $\mathrm{Ni}$, respectively. The time of the solidification process is gradually decreasing with the reduction of temperature to 0.8 and $0.7 T_{m}$. Owing to the fact that these simulations are relatively close to the melting temperature, the formation and dissolution of solid-like structures "embryos" is still possible, but without a direct impact on the FLV. (2) The difference between in the results obtained in the two series of simulations emerges at $0.6 T_{m}$. Although the solidification process for EAM-Johnson $\mathrm{Ni}$ is completed within $70 \mathrm{ps}$ as it can be seen from the steep slope of the corresponding line in Fig. 4.13(b), the same process takes more than $160 \mathrm{ps}$ in the EAM-FBD Ni simulation, where the slope is gradually changing after $70 \mathrm{ps}$ from the quenching time, Fig. 4.13(a). The change in the slope of the FLV in the EAM-FBD Ni simulation is mainly related to the nucleation and the fast growth of the solid structures inside the liquid phase, which progressively delays the decrease of the melted region. (3) In the low temperature region, from 0.5 down to $0.1 T_{m}$, the reduction in the FLV, in EAM-Johnson Ni is approximately similar to those observed at 0.6 and $0.7 T_{m}$, Fig. 4.13(b). In all these simulations, the time needed for complete solidification is approximately $70 \mathrm{ps}$, which indicates that the velocity of the solidification front remains within almost the same value in all these simulations. On the other hand, the decrease in the FLV for the EAM-FBD Ni at 0.5 down to $0.1 T_{m}$, is similar to the ones at 0.6 and $0.7 T_{m}$ during the first $50 \mathrm{ps}$ and then the divergence between the FLV results takes place due to the nucleation and growth of the crystalline structures in these simulations, Fig. 4.13(a).

In order to support our hypothesis about the impact of the homogeneous nucleation on the advancement of the solidification front, the average instantaneous velocity (AIV) of the solidification front, along with the average fraction of crystalline regions in the liquid phase adjacent to the interfaces (AFC) have been analysis for every 5 ps for the two series of MD simulations for Ni system. The main purpose from this analysis is to determine the influence of the nucleation of the crystalline structures, which are formed close to the liquid-solid interface, on the movement of the solidification front. Figures $4.14,4.15$, and 4.16 show the results of this analysis for three representative target temperatures: $0.8 T_{m}$ represents the simulations in the high temperature region, $0.6 T_{m}$ is the vertex of the $T T T$ diagram, and $0.3 T_{m}$ represents the low temperature region. 

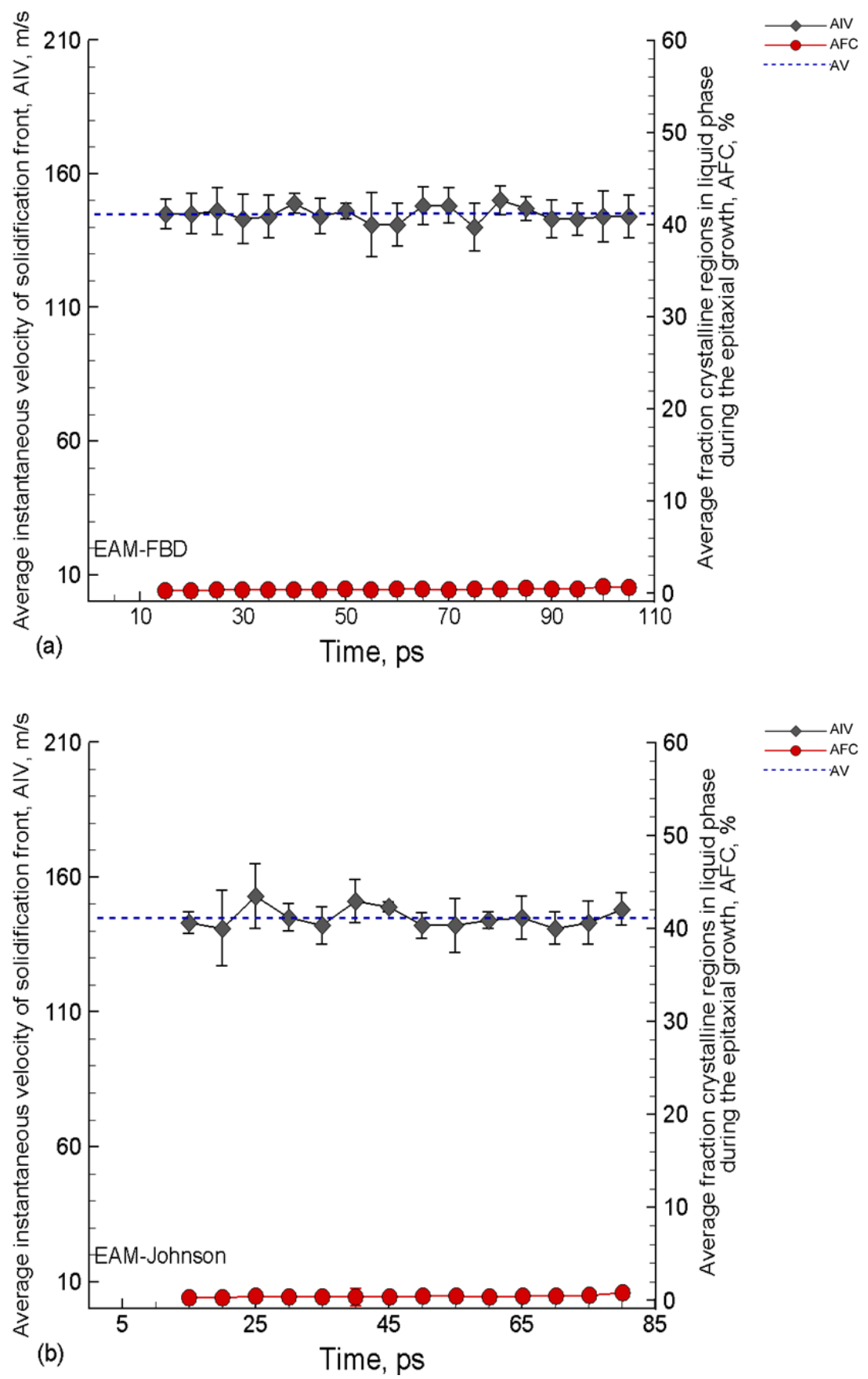

Fig. 4.14. The evolution of the average instantaneous velocity of solidification front (AIV) and average fraction of crystalline regions in the liquid phase (AFC) during the solidification of $\mathrm{Ni}$ systems represented by (a) EAM-FBD and (b) EAM-Johnson potentials at a target temperature $0.8 T_{m}$. The error bars in the AIV and AFC represent the standard deviations obtained from three series of MD simulations, each starting with different initial conditions. The dashed blue line represents the average instantaneous velocity during the simulation time. 
In each example, we compare the results obtained in MD simulations for EAM-FBD Ni and EAM-Johnson Ni. The error bars in all these figures represent the standard deviation for AIV and AFC obtained from three series of MD simulations, each starting with different initial conditions. The blue dashed lines represent the average instantaneous velocity during the time of solidification.

In the simulations performed with both potentials at $0.8 T_{m}$, the instantaneous displacement, $\sim 0.6 \mathrm{~nm}$ for every $5 \mathrm{ps}$ or $145 \mathrm{~m} / \mathrm{s}$, and the instantaneous AFC, fluctuating between $0.3 \%$ and $0.8 \%$, remain roughly constant during the solidification process, Fig. 4.14 (a) and (b). The influence of the AFC appears clearly on the AIV of the solidification front in the MD simulations performed at $0.6 T_{m}$, as can be seen from Fig. 4.15. For EAM-FBD Ni, the impact of the nucleation of the crystalline structures on the velocity of the liquid-solid interface becomes obvious at 30 ps. During the time between 25 ps to $30 \mathrm{ps}$, the fraction of the crystalline structures increase from $2 \%$ to $3.2 \%$, resulting in the decrease of the instantaneous velocity from $167 \mathrm{~m} / \mathrm{s}$ to $154 \mathrm{~m} / \mathrm{s}$. Within the next $10 \mathrm{ps}$ the velocity further decreases to $\sim 134 \mathrm{~m} / \mathrm{s}$ as the fraction of the crystalline regions increases to $\sim 8 \%$. As can be seen from Fig. 4.15(a), for every 5 ps, the increase in the AFC near the interface region correlates directly with the reduction in the velocity of the advancing solidification front. At the end of the simulation, the results illustrated that between 15 and $140 \mathrm{ps}$, the reduction in the instantaneous velocity from 181 to $25 \mathrm{~m} / \mathrm{s}$ coincides with the increase in the fraction of the crystalline regions near the interface from $0.8 \%$ to $\sim 50 \%$. The average instantaneous velocity is $82.2 \mathrm{~m} / \mathrm{s}$.

Although the results obtained for the solidification temperature of $0.6 T_{m}$ with EAMJohnson potential are very different from the ones discussed above for the EAM-FBD Ni, but they strongly support our hypothesis on the role of the homogeneous nucleation in the slowdown of the solidification front. In this simulation, the instantaneous velocity of the solidification front oscillates between $186 \mathrm{~m} / \mathrm{s}$ and $170 \mathrm{~m} / \mathrm{s}$ during the first $30 \mathrm{ps}$ of the simulation, the fraction of crystalline regions close to the interface slowly increases from $0.56 \%$ and $0.95 \%$. The small increase in the fraction of crystalline regions does not impact on the instantaneous velocity of solidification front and the velocity fluctuated around $174 \mathrm{~m} / \mathrm{s}$ between $25 \mathrm{ps}$ and $45 \mathrm{ps}$ at the time when the fraction of the crystalline regions further increases from $0.8 \%$ to $2 \%$. 

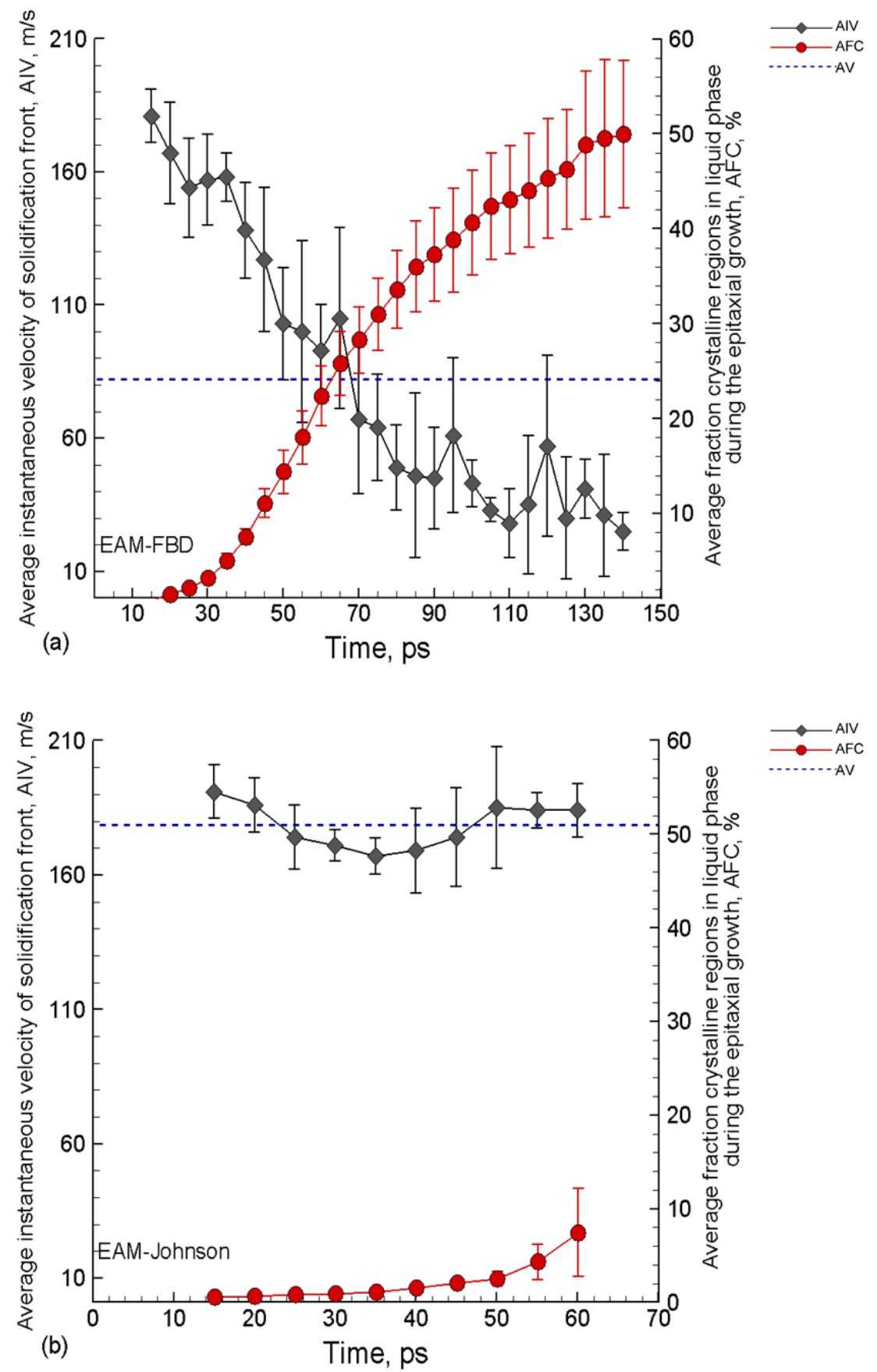

Fig. 4.15. The evolution of the average instantaneous velocity of solidification front (AIV) and average fraction of crystalline regions in the liquid phase (AFC) during the solidification of $\mathrm{Ni}$ systems represented by (a) EAM-FBD and (b) EAM-Johnson potentials at a target temperature $0.6 T_{m}$. The error bars in the AIV and AFC represent the standard deviations obtained from three series of MD simulations, each starting with different initial conditions. The dashed blue line represents the average instantaneous velocity during the simulation time. 
The fraction of the crystalline regions reaches its maximum of $7 \%$ by the end of the simulation and does not affect the velocity of the solidification front, which remains at $\sim 184 \mathrm{~m} / \mathrm{s}$. Just for the clarification and as mentioned earlier, the calculated values of the instantaneous velocity and the fraction of the crystalline regions are the average values obtained from three series of MD simulations, each starting with different initial conditions. For instance, in the EAM-Johnson $\mathrm{Ni}$ simulations at $0.6 T_{m}$, the formation of a small individual crystalline structure inside the liquid phase at $40 \mathrm{ps}$, as it presented in Fig. 4.10(b), was not observed in the other two EAM-Johnson Ni simulations at the same temperature. As a result, the average fraction of the crystalline regions does not show the real influence on the average instantaneous velocity of the solidification front. These results are in contrast with the ones obtained from EAM-FBD Ni simulations at $0.6 T_{m}$, where the nucleation and fast growth of the crystalline structures inside the liquid phase appears immediately after quenching to the target temperature in all three simulations, and the fraction of the crystalline structures has a significant impact on the average instantaneous velocity of the solidification front.

The analysis of the results obtained from series of MD simulations for $\mathrm{Ni}$ system at $0.3 T_{m}$, which is below the glass transition temperature for $\mathrm{Ni}$, are presented in Fig. 4.16. For EAM-FBD Ni, Fig. 4.16(a), the nucleation of crystalline structures near the liquid-solid interface is noticed after $20 \mathrm{ps}$ from the quenching time, when the AFC slowly grows from $0.7 \%$ to $1.5 \%$ during the first $15 \mathrm{ps}$ and the instantaneous velocity decelerates from $174 \mathrm{~m} / \mathrm{s}$ to $153 \mathrm{~m} / \mathrm{s}$. A combination of two factors plays a role in the deceleration of the instantaneous velocity of the interface at this early stage of the solidification process, the nucleation of crystalline regions near to the interface and the reduction of the atomic mobility in this simulation if compared to the ones at high temperature region. The effect of the average fraction of the crystalline regions close to the liquid-solid interface progressively dominates during the last $20 \mathrm{ps}$ of the simulation due to the reduction in the liquid volume and the existence of the solid structure, where the AFC increases from $0.7 \%$ to $\sim 5 \%$ during the $85 \mathrm{ps}$. The results for EAM-Johnson $\mathrm{Ni}$ at $0.3 T_{m}$, Fig. 4.16(b), on the other hand, show the minimal effect of the low fraction of the crystalline regions on the solidification front velocity. The velocity oscillates between 160 and $190 \mathrm{~m} / \mathrm{s}$ during the $60 \mathrm{ps}$ of the simulation time, whereas the average fraction of the crystalline regions reaches $\sim 2.5 \%$ by the end of the simulation. 

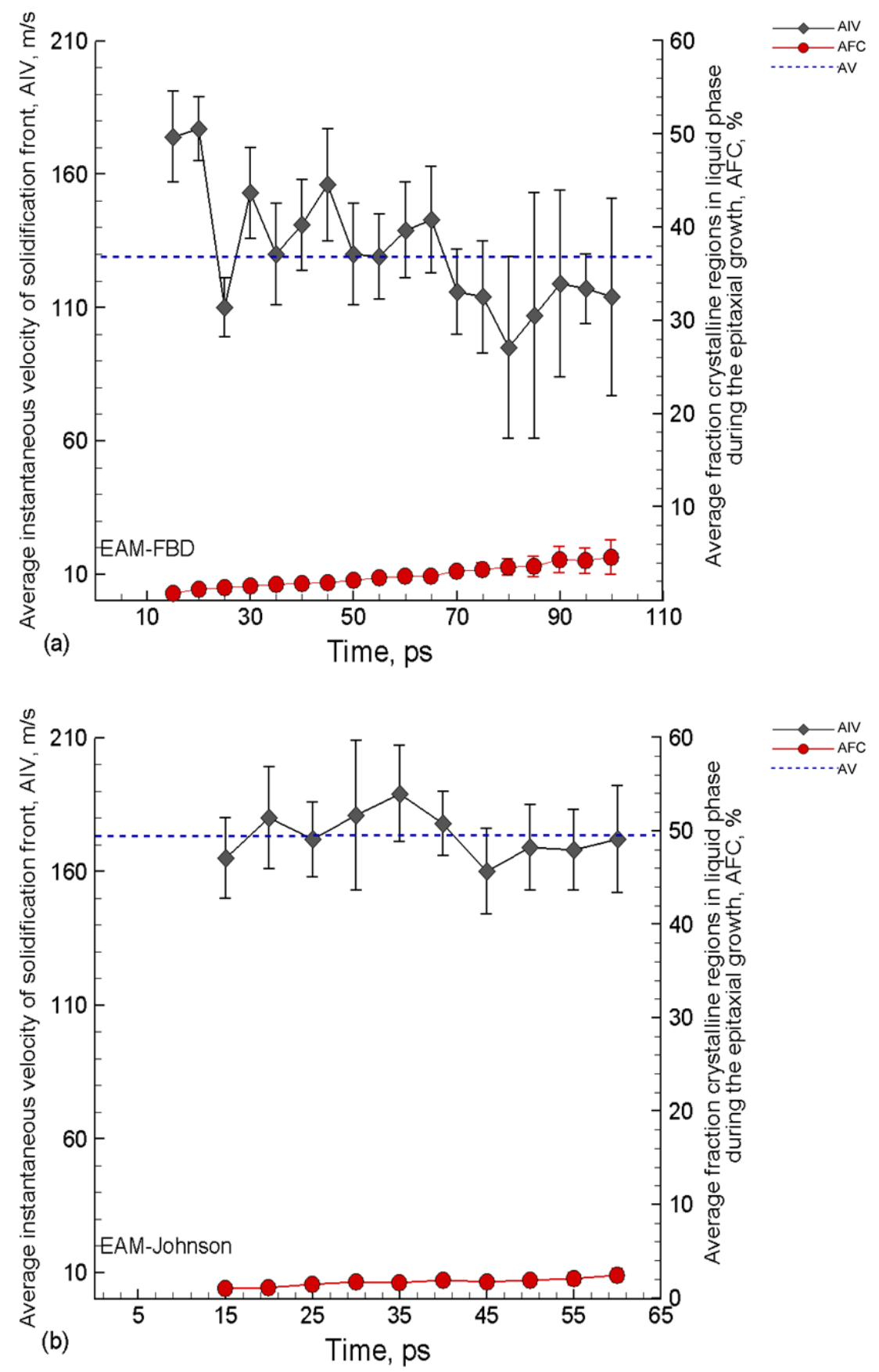

Fig. 4.16. The evolution of the average instantaneous velocity of solidification front (AIV) and average fraction of crystalline regions in the liquid phase (AFC) during the solidification of $\mathrm{Ni}$ systems represented by (a) EAM-FBD and (b) EAM-Johnson potentials at a target temperature $0.3 T_{m}$. The error bars in the AIV and AFC represent the standard deviations obtained from three series of MD simulations, each starting with different initial conditions. The dashed blue line represents the average instantaneous velocity during the simulation time. 
The small effect of the crystalline structures on the advancement of the liquid-solid interface is reflected in a relatively large average velocity of the interface, $173 \mathrm{~m} / \mathrm{s}$, as compared to smaller value of $\sim 129 \mathrm{~m} / \mathrm{s}$ predicted for EAM-FBD Ni.

The comprehensive picture of the microscopic correlation between the nucleation of crystalline structures and the advancement of the solidification front emerging from analysis of the results of MD simulations performed for $\mathrm{Ni}$ with the two different potentials is depicted in Fig. 4.17, where the instantaneous velocity of the solidification front is plotted with respect to the normalized temperature for (a) EAM-FBD Ni and (b) EAM-Johnson Ni. The plots are divided into three regimes that describe the main characteristics that define the mobility of the solidification front: (1) solidification, (2) homogeneous nucleation, and (3) amorphous. For EAM-FBD Ni, Fig. 4.17(a), the solidification regime extends from the melting temperature down to $0.70 T_{m}$. The advancement of the solidification front in this regime is mainly controlled by the undercooling condition, $\Delta T$, which defines the thermodynamic driving force for the solidification process. In this regime, the results failed to provide any evidence of the formation of stable crystal nuclei inside the liquid phase due to the large energy barrier for the nucleation, while the formation and dissociation of sub-critical embryos do not influence the movement of the solidification front.

In the homogeneous nucleation regime, the nucleation and growth of crystalline structures are clearly observed in several simulations performed in a temperature range between 0.65 and $0.25 T_{m}$. The effect of the nucleation of crystalline structures on the instantaneous velocity of the solidification front can be illustrated by measuring the velocity at different stages of the solidification process. First, the instantaneous velocity of the solidification front is measured before the nucleation of the crystalline structures, yielding the values between 125 and $175 \mathrm{~m} / \mathrm{s}$ shown as gray diamonds in Fig. 4.17(a). In the simulation performed at 0.60 and 0.55 $T_{m}$, the fast nucleation of the crystalline structures, combined with the exclusion of the first $10 \mathrm{ps}$ from the analysis, prevents us from finding the initial instantaneous velocity. Second, the instantaneous velocity is measured during the time when the fraction of crystalline structures inside the liquid phase reaches $3 \%$. 

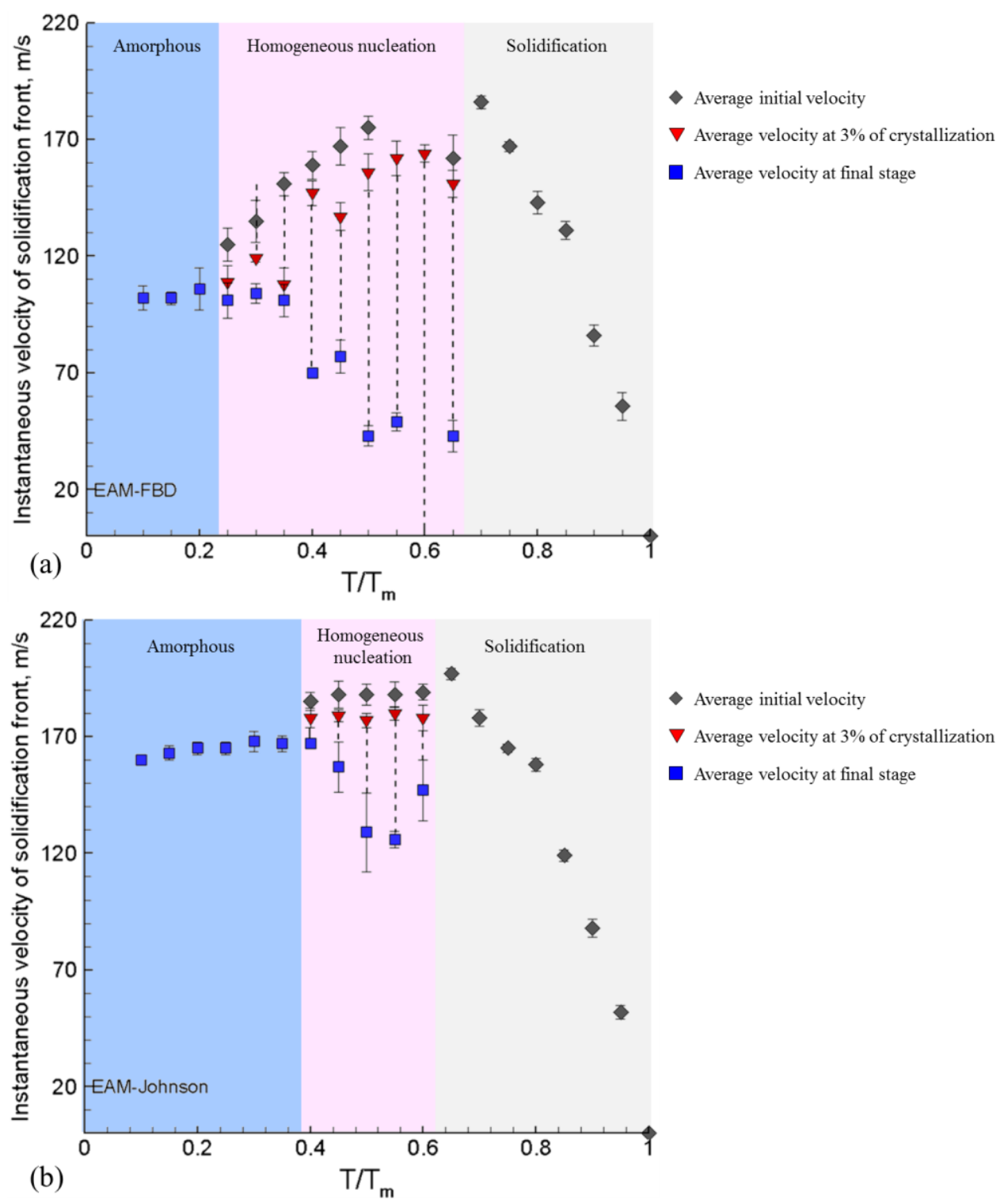

Fig. 4.17. Instantaneous velocities of the solidification front with respect to the normalized temperature obtained from MD simulations of solidification of Ni systems described by (a) EAM-FBD and (b) EAMJohnson potentials. Each diagram is divided into three regimes, depending on the major factors affecting the advancement of the solidification front: solidification, homogeneous nucleation, and amorphous regimes. The gray diamonds show the velocity of the solidification front in the regions of undercooled liquid that are free of any crystalline nuclei. The red delta symbols represent the velocity of solidification front at a time when the fraction of crystalline structures in the liquid phase reach $3 \%$. The blue square symbols represent the solidification front velocity during the last $10 \mathrm{ps}$ of the simulation time. The error bars represent the standard deviation obtained from three series of MD simulations, each starting with different initial conditions. 
The effect of the nucleation of the crystalline structures on the solidification front velocity leads to the reduction of the instantaneous velocity from its initial levels at the first stage of the solidification process to lower values ranging between 108 and $164 \mathrm{~m} / \mathrm{s}$, as shown by red delta symbols in Fig. 4.17(a). Finally, the instantaneous velocity is measured during the last 10 ps of the solidification process in order to determine the impact of the evolution of the crystalline structures on the solidification front velocity. The results illustrate further deceleration of the solidification front, with the final instantaneous velocities ranging between 43 and $104 \mathrm{~m} / \mathrm{s}$, as depicted by blue square symbol in Fig. 4.17(a).

The amorphous regime represents the simulations where the temperature ranges between 0.20 and $0.10 T_{m}$ and is characterized by the formation of some small crystallites structures inside the liquid phase after $\sim 20$ ps. These structures remain almost at the same positions, slightly grow in the course of the simulations, and are finally absorbed by the advancing solidification front. The concentration of these structures increased during the final stage of the solidification process and results in the reduction of the instantaneous velocity of the solidification front during the last $10 \mathrm{ps}$ of the simulation to $\sim 102 \mathrm{~m} / \mathrm{s}$.

The same analysis is carried out for the EAM-Johnson $\mathrm{Ni}$ and the results are shown in Fig. 4.17(b). Although the curve of the solidification front is divided into the same three regimes discussed above for EAM-FBD $\mathrm{Ni}$, the ranges of temperatures and the values of the solidification front velocity are different in EAM-Johnson Ni. The range of temperatures for the solidification regime is extended to a lower temperature of $0.65 T_{m}$, where the velocity is reaching the maximum value of $197 \mathrm{~m} / \mathrm{s}$. In contrast to EAM-FBD Ni, the fraction of crystalline regions in the liquid phase remains below $0.6 \%$ during the time of the simulation and, therefore, the data point for $0.65 T_{m}$ is assigned to the solidification regime for EAM-Johnson Ni. In the following five simulations performed at $0.6,0.55,0.50,0.45$, and $0.40 T_{m}$ the fraction of crystalline structures reaches $3 \%$ after 40 to 50 ps of the simulations, which results in a slight decrease of the velocity of the solidification front, from $\sim 180$ to $177 \mathrm{~m} / \mathrm{s}$. The subsequent growth of these structures at a later time leads to further deceleration of the interface, down to the velocities between 126 and $167 \mathrm{~m} / \mathrm{s}$ in the last 10 ps of the solidification process. Finally, in the amorphous regime, we observe the formation of some solid structures in six simulations performed at 0.35 , $0.30,0.25,0.20,0.15,0.10 T_{m}$, with the fraction of crystalline structures remaining below $2 \%$. Due to the low concentration of these solid structures in the liquid phase, the velocity of the 
solidification front is not strongly affected by their presence and is ranging between 168 and 160 $\mathrm{m} / \mathrm{s}$ during the last $10 \mathrm{ps}$ of the simulations. These velocity values ranged are higher than the ones observed in the EAM-FBD Ni.

The homogeneous nucleation of crystalline structures inside the liquid phase is also observed in two simulations in EAM Cr, at 0.60 and $0.65 T_{m}$. Due to the low fractions of these structures, however, they do not affect on the movement of the solidification front in any significant way and we neglect their presence in our analysis of the simulation results.

Finally, the concentration of vacancies is also investigated in the series of MD simulations performed for EAM-FBD Ni and the results are compared to the ones obtained for EAM-Johnson Ni in Fig. 4.18. The concentration of vacancies is measured only in the regions solidified through the movement of solidification front and all the regions where the generation of vacancies is affected by the homogeneous nucleation of crystalline structures inside the liquid phase are excluded from the analysis. Similar to what has been observed in EAM-Johnson Ni, the concentration of vacancies is increasing with the increase of the undercooling and reaches the maximum value of $0.25 \%$ at $0.10 T_{m}$. This value is almost twice the maximum value of the concentration of vacancies in EAM-Johnson Ni, $0.13 \%$ at $0.10 T_{m}$. Two factors may explain the difference in the concentration of vacancies between the two systems:

(1) The difference in the vacancy formation energy between the two systems, where our calculation shows that the vacancy formation energies are $1.63 \mathrm{eV}$ and $1.69 \mathrm{eV}$ for EAM-FBD $\mathrm{Ni}$ and EAM-Johnson Ni, respectively, which is consistent with Ref. [128, 187];

(2) The difference in the melting temperature between the two systems which results in different actual undercooling temperatures if the two system are compared for the same nominal undercooling. For instance, the temperature of $0.60 T_{m}$ in EAM-Johnson is equal to $867 \mathrm{~K}$ while in EAM-FBD it is $1031 \mathrm{~K}$. This difference in the undercooling temperatures, along with the difference in the vacancy formation energy, could be the reasons responsible for difference in the concentration of vacancies in $\mathrm{Ni}$ described by different interatomic potentials. 

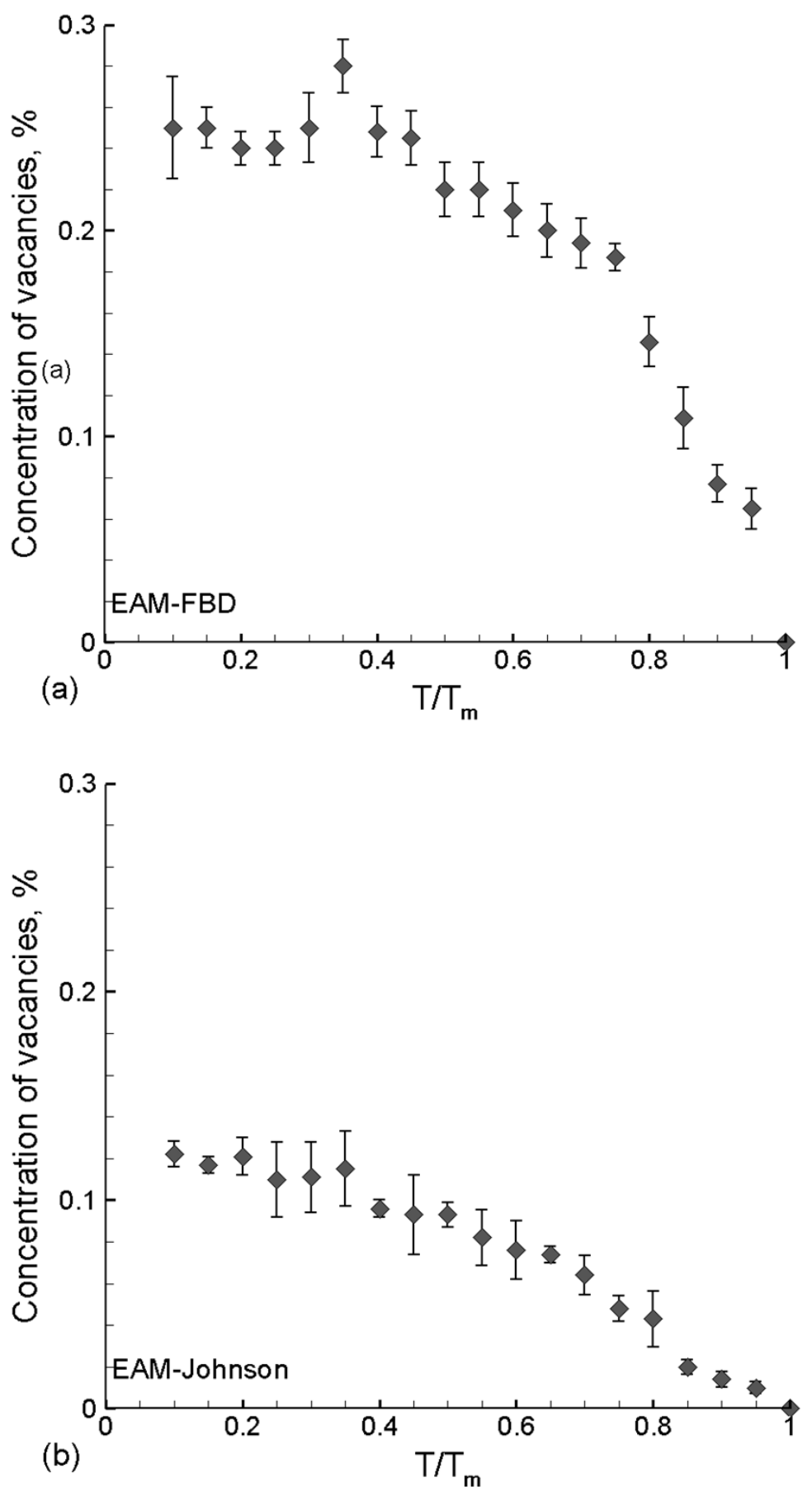

Fig. 4.18. Concentration of vacancies as a function of normalized temperature in Ni systems described by (a) EAM-FBD and (b) EAM-Johnson potentials. The error bars represent the standard deviation obtained from three series of MD simulations, each starting with different initial conditions. 


\subsection{Summary}

A series of MD simulations of solidification in $\mathrm{Cr}$ and $\mathrm{Ni}$ systems is performed with the aim of revealing the mechanisms responsible for the vacancies formation in bec and fcc crystal structures. The solidification process is simulated under well-controlled temperature and pressure conditions and the distributions of vacancies are analyzed in each atomic plane and related to the velocity of the solidification front. The detailed structural analysis of the simulation results demonstrate that the overwhelming majority of vacancies in $\mathrm{Cr}$ and all vacancies in $\mathrm{Ni}$ are generated in the regions that experienced melting and solidification process. Furthermore, the results illustrate a strong correlation between the vacancy concentration and the velocity of solidification front. For instance, in the $\mathrm{Cr}$ system, the concentration of vacancies increases as the solidification front velocity increases and decreases when with the solidification front decelerates. Close up investigation of the interface region is also undertaken to determine the dominant mechanism responsible for the vacancy formation. The vacancy diffusivity as well as the time between the vacancy jumps are determined for each system. The simulations reveal that the dominant mechanism responsible for the vacancies formation in both systems is the generation of vacant lattice sites at the rapidly advancing solidification front during the solidification process.

The effect of homogeneous nucleation of new crystallites in the supercooled liquid on the velocity of the solidification front is also investigated in a series of MD simulations where the interatomic interaction in the Ni system is described by two different potentials, EAM-FBD and EAM-Johnson. In both cases, the curves of the solidification front velocity with respect to the normalized temperature show linear dependences in the high temperature regime, from the melting temperature down to $0.7 T_{m}$. This behavior changes in the low temperature regime. While the velocity predicted for EAM-FBD Ni decreases with the reduction of temperature, the velocity predicted for EAM-Johnson Ni remains constant down to a temperature of $0.1 T_{m}$. The results for EAM-FBD Ni illustrate a prominent effect of the nucleation of crystalline structures inside the liquid phase on the advancement of the solidification front. 


\section{Chapter 5}

\section{The effect of spatial confinement by transparent overlayer on the laser melting, photomechanical spallation, and phase explosion of metal targets irradiated by short laser pulse}

The results and discussion in this chapter are reported in two journal papers:

1- The results in section 5.1 are reported in Journal Applied Physics:

E. T. Karim, M. Shugaev, C. Wu, Z. Lin, R. F. Hainsey, and L. V. Zhigilei, Atomistic simulation study of short pulse laser interactions with a metal target under conditions of spatial confinement by a transparent overlayer, J. Appl. Phys.115, 183501, 2014.

- The TTM-MD simulations of Ag target irradiated in vacuum by a 10 ps laser pulse at an absorbed laser fluencies of 900,1000, $3000 \mathrm{~J} / \mathrm{m}^{2}$ are performed and analyzed by Mr. Maxim V. Shugaev.

2- A paper reporting the results in section 5.2 is submitted to the Applied Physics A:

E. T. Karim, M. V. Shugaev, C. Wu, Z. Lin, H. Matsumoto, M. Conneran, J. Kleinert, R. F. Hainsey, and L. V. Zhigilei, Experimental characterization and atomistic modeling of interfacial void formation and detachment in short pulse laser-processing of metal surfaces covered by transparent overlayers, Appl. Phys. A, submitted.

- The results reported in section 5.2.2, "Experimental characterization of laser modification of metal-overlayer targets" are obtained by a research team from Electro Scientific Industries, Inc.

- The TTM-MD simulation in section 5.2.3.3: "The effect of pre-existing interfacial voids" is performed and analyzed by Mr. Maxim V. Shugaev. 


\subsection{Atomistic simulation study of short pulse laser interactions with a metal target under conditions of spatial confinement by a transparent overlayer}

\subsubsection{Introduction}

While the general mechanisms of short pulse laser melting, spallation, and ablation of metals in vacuum and in a background gas have been extensively studied experimentally, theoretically, and computationally, the effect of strong spatial confinement of the laser-induced processes by a solid transparent overlayer remains largely unexplored. The spatial confinement is realized when a metal target (a film or a bulk substrate) is covered by a transparent overlayer and the laser irradiation is focused on the metal surface through the overlayer. This setup has been shown to be advantageous for a number of practical applications, from laser shock peening [215-218] to laser-induced forward transfer [219-221] and thin film patterning/scribing, [222, 223] and to the generation of high-quality surface nanostructures [57]. In particular, a substantial increase in pressure generated in laser ablation confined by a glass overlayer [215, 216, 224] has been utilized in material processing by laser-induced shock waves [215-218] and has enabled design of "exotic targets" with enhanced momentum transfer for laser propulsion applications [225]. Recently, the generation of periodic nanostructures by multiple beam interference has been reported for $\mathrm{Ni}$ and $\mathrm{Si}$ surfaces covered by optically transparent $400 \mathrm{~nm}$ thick polymer layers [57]. A significant improvement in the quality of the nanostructured surfaces, as compared to processing of bare surfaces, is observed and attributed to the suppression of the expansion and breakup of molten material as well as trapping of the ejected droplets by the overlayer.

The analytical description and computer modeling of laser-induced processes under conditions of spatial confinement have been largely focused on thermodynamic analysis of the evolution of pressure exerted on the metal target by the vapor/plasma plume expansion and evaluation of the momentum transfer to the irradiated target [216, 223-225]. In several recent numerical simulations of thin film removal by backside femtosecond pulse irradiation through a transparent substrate, the dynamics of the relaxation of thermo-elastic stresses and the mechanical deformation of the metal film are considered [218, 226, 227] and found to play the dominant role in the separation and ejection of the film fragments at laser fluences close to the ablation threshold [226]. The analysis of the effect of the spatial confinement on the kinetics and mechanisms of laser-induced phase transformation, as well as the structural modification of the 
metal target, however, is beyond the capabilities of the continuum-level finite difference [227] or finite element method [218, 226] calculations. On the other hand, the atomistic molecular dynamics (MD) simulations, while more computationally expensive, have been shown to be capable of providing detailed information on the fast non-equilibrium structural and phase transformations induced by short pulse laser irradiation of thin films[228-231, 152, 161, 162] and bulk targets $[24,113,120,129,134,146,152,232,233]$ under vacuum conditions or in the presence of background gas [234].

\subsubsection{Computational model}

The computational setups used in the simulations of laser interactions with a bulk $\mathrm{Ag}$ target covered by a transparent overlayer are schematically illustrated in Fig. 5.1. The two components of the computational setup, the model for simulation of laser interaction with the metal target and the representation of the transparent overlayer, are described below.

\subsubsection{TTM-MD model for Ag target}

The laser interaction with the bulk Ag target is simulated with a model that combines the classical MD method with the two-temperature model (TTM) [90] that describes the evolution of lattice and electron temperatures by two coupled nonlinear differential equations and accounts for the laser excitation of the conduction band electrons, electron-phonon energy exchange and electron heat conduction. In the combined TTM-MD model, MD substitutes the TTM equation for the lattice temperature and the diffusion equation for the electron temperature is solved simultaneously with MD integration of the equations of motion of atoms. The cells introduced to solve the TTM equation for the electron temperature are related to the corresponding regions of the MD system. The local lattice temperature is then defined for each cell from the average kinetic energy of thermal motion of atoms. A complete description of the TTM-MD model is given in Ref. [118] and below we only delineate the computational setup and provide the parameters of the model used in the simulations reported in this section. 


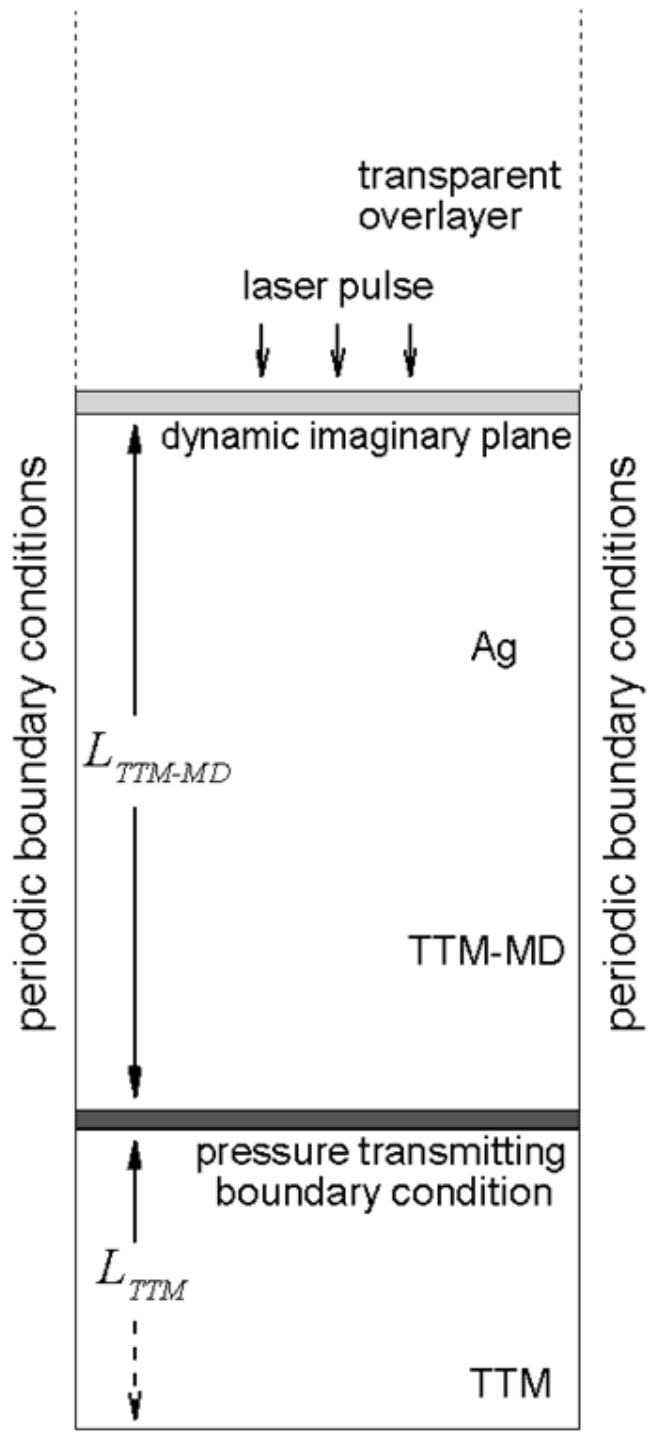

(a)

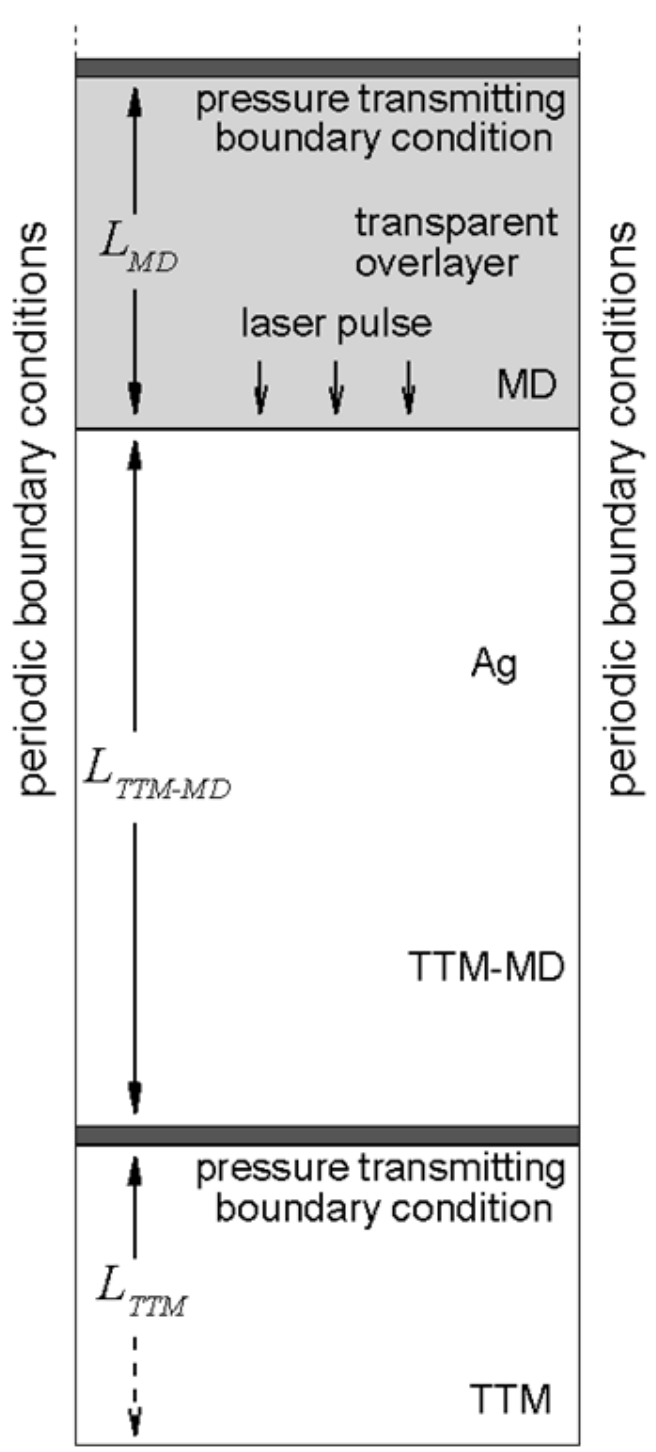

(b)

Fig. 5.1. Schematic sketches of two alternative computational setups designed for simulations of laser interactions with a $\mathrm{Ag}$ target covered by a transparent overlayer. In both setups, the top layer of the $\mathrm{Ag}$ target with thickness of $L_{T T M-M D}$ is represented by the atomistic TTM-MD model, whereas the heat conduction in the deeper part of the substrate is simulated with TTM equations solved in the region with thickness of $L_{T T M}$. The transparent overlayer is represented by a dynamic imaginary plane in (a) and by a model adopting an atomistic MD description of a part of the overlayer adjacent to the Ag target in (b). The dynamic pressure-transmitting boundary conditions are applied at the bottom of the TTM-MD region and, in (b), at the top of the MD part of the overlayer to mimic non-reflective propagation of laserinduced pressure waves through the boundaries. In both setups, the partial propagation of the laserinduced pressure wave into the silica overlayer and the work of adhesion between the overlayer and the $\mathrm{Ag}$ film are reproduced. 
The interatomic interactions in the Ag target is described by the embedded atom method (EAM) potential in the form proposed by Foiles, Baskes, and Daw (FBD) [128]. A cut-off function [209] is added to the potential to smoothly bring the interaction energies and forces to zero at interatomic distance of $5.5 \AA$. Although the EAM- FBD potential is fitted to lowtemperature values of the equilibrium lattice constant, sublimation energy, bulk modulus, elastic constants, and vacancy formation energy, it also provides a good description of high-temperature thermodynamic properties of Ag relevant to the conditions of laser-induced melting and ablation. In particular, the equilibrium melting temperature, $T_{m}$, determined in liquid-crystal coexistence MD simulations is $1139 \mathrm{~K}$ [141] about $8 \%$ below the experimental value of $1235 \mathrm{~K}$ [235]. The temperature and pressure conditions for the onset of the explosive phase separation into liquid and vapor, $T^{*}$ and $P^{*}$, are determined for the FBD EAM Ag in a series of constant-pressure MD simulations of a slow $(0.1 \mathrm{~K} / \mathrm{ps})$ heating of a metastable liquid, following an approach applied earlier to other material systems $[120,134,166,167]$. The onset of the phase explosion is identified from a sharp increase in volume of the system and the threshold temperature for the phase explosion is found to be about $3450 \mathrm{~K}$ at zero pressure and about $4850 \mathrm{~K}$ at $0.5 \mathrm{GPa}$. The onset of the phase explosion can be expected at $\sim 10 \%$ below the critical temperature [23, 145 , 166] and the values of $T^{*}$ calculated for EAM- FBD are not in conflict with the broad range of experimental values of the critical temperature of Ag spanning from $4300 \mathrm{~K}$ to $7500 \mathrm{~K}$ [236]. At negative values of pressure, the onset of cavitation in the metastable liquid is observed at about $2060 \mathrm{~K}$ at $-0.5 \mathrm{GPa}, 1530 \mathrm{~K}$ at $-1 \mathrm{GPa}$, and $1400 \mathrm{~K}$ at $-1.5 \mathrm{GPa}$.

The choice of the parameters of the TTM equation for the electron temperature for Ag is the same as in Ref. [141], where a more detailed explanation of the parameters is provided. The electron temperature dependences of the electron-phonon coupling factor and electron heat capacity are taken in the forms that account for the thermal excitation from the electron states below the Fermi level [86]. The temperature dependence of the electron thermal conductivity is described by the Drude model relationship, with parameters evaluated based on the free electron model and the experimental value of the thermal conductivity of Ag [141].

The irradiation of the target with a 10 ps laser pulse is represented through a source term added to the TTM equation for the electron temperature [118]. The source term simulates excitation of the conduction band electrons by a laser pulse with a Gaussian temporal profile and reproduces the exponential attenuation of laser intensity with depth under the surface. To account 
for the energy transport occurring before the thermalization of the excited electrons, the optical absorption depth, $L_{p}=12 \mathrm{~nm}$ at laser wavelength of $1 \mu \mathrm{m}$, [13] is combined with the effective depth of the "ballistic" energy transport, $L_{b}=56 \mathrm{~nm}$, roughly estimated here as a product of the Fermi velocity and the Drude relaxation time [122]. To ensure complete deposition of the energy of the 10 ps laser pulses, the peak intensity of the Gaussian laser pulse is shifted to 25 ps from the beginning of the simulations. The absorbed laser fluence, $F_{a b s}$, rather than the incident fluence is used in the discussion of the simulation results.

As illustrated in Fig. 5.1, the atomic-level TTM-MD representation is used only for the top part of the Ag target, where the laser-induced structural modifications take place. The systems with different depth of the TTM-MD region, $L_{T T M-M D}$, are used at different laser fluences, namely, $L_{T T M-M D}=209 \mathrm{~nm}$ for $F_{a b s}=900 \mathrm{~J} / \mathrm{m}^{2}$ and $F_{a b s}=1000 \mathrm{~J} / \mathrm{m}^{2}$, $L_{T T M-M D}=411 \mathrm{~nm}$ for $F_{a b s}=3000 \mathrm{~J} / \mathrm{m}^{2}$, and $L_{T T M-M D}=699 \mathrm{~nm}$ for $F_{a b s}=6000 \mathrm{~J} / \mathrm{m}^{2}$. The heat transfer in the deeper part of the target is described by the TTM equations for lattice and electron temperatures solved down to the depth of $L_{T T M-M D}+L_{T T M}$, with $L_{T T M}=2.5 \mu \mathrm{m}$ chosen to ensure the absence of any significant increase in the electron or lattice temperatures at the bottom of the computational domain by the end of the simulation.

The lateral (parallel to the surface of the Ag target or the interface with the overlayer) dimensions of the computational systems are $4.1 \times 4.1 \mathrm{~nm}^{2}$, and the number of $\mathrm{Ag}$ atoms in the TTM-MD parts of the systems is 203600 for $L_{T T M-M D}=209 \mathrm{~nm}, 399600$ for $L_{T T M-M D}=411 \mathrm{~nm}$, and 680000 for $L_{T T M-M D}=699 \mathrm{~nm}$. The periodic boundary conditions are imposed in the lateral directions to reproduce the interaction of the atoms with the surrounding material. This approach is appropriate when the laser spot diameter is much larger than the depth of the laser energy deposition, so that any effects related to the lateral variations of the irradiation and thermal conditions can be neglected and the simulated part of the system remains laterally confined by the surrounding material during the time of the simulation. At the bottom of the TTM-MD part of the model, a special pressure-transmitting [110, 130] heat-conducting boundary condition is applied in order to avoid an artificial reflection of the laser-induced pressure wave and to account for the energy transfer from the surface region of the metal target, represented with atomic-level resolution, to the deeper part of the target, represented at the 
continuum level. The simulations are performed for systems with free metal surfaces exposed to the laser irradiation as well as for metal targets covered by thick transparent overlayers. The computational representation of the overlayers in the model is described next.

\subsubsection{Computational representation of the transparent overlayer}

Two different methods developed in this work for representation of the transparent overlayer are illustrated in Fig. 5.1. In both implementations, the properties of fused silica are used in parameterization and the overlayer is assumed to be sufficiently thick to ensure that the pressure wave generated in the overlayer due to the laser energy absorption by the metal target does not reflect from the top surface of the overlayer and come back to the metal-overlayer interface during the time of the simulation. For a $10 \mu \mathrm{m}$ thick overlayer and the speed of sound of $5900 \mathrm{~m} / \mathrm{s}$ in fused silica [235], it takes more than $3 \mathrm{~ns}$ for the reflected wave to reach the interface. Under irradiation regime considered in this work (from the threshold for surface melting up to several times the threshold), the time scale of laser-induced structural and phase transformations does not exceed several nanoseconds and the reflected pressure wave would not affect the transformations for overlayer with thickness on the order of tens of micrometers.

In the first method, illustrated in Fig. 5.1(a), the overlayer is not represented with atomic resolution but introduced through a dynamic acoustic impedance matching boundary condition that accounts for the displacement of the metal-overlayer interface in response to the thermal expansion and phase transformations occurring in the surface region of the metal target. The dynamic boundary condition in this case takes the form of an imaginary plane that interacts with the Ag atoms through the Lennard-Jones ( $\mathrm{LJ}$ ) potential defined as a function of the distance between an Ag atom and the imaginary plane. The imaginary plane moves in response to forces

acting on it from the Ag atoms as well as an additional force $\vec{F}_{e}$ that mimics the elastic response of the overlayer to forces acting from the side of the metal target. This additional force is proportional to the instantaneous velocity $\vec{v}$ of the imaginary plane and acts in the opposite direction, $\vec{F}_{e}=-S z \vec{v}$, where $S$ is the surface area of the interface and $z$ is the acoustic impedance of the overlayer material. For the silica overlayer, the acoustic impedance is calculated as $z=\rho_{\mathrm{SiO}_{2}} c_{\mathrm{SiO}_{2}}$ where $\rho_{\mathrm{SiO}_{2}}=2.2 \mathrm{~g} / \mathrm{cm}^{3}$ and $c_{\mathrm{SiO}_{2}}=5900 \mathrm{~m} / \mathrm{s}$ are the experimental values of density and speed of sound in fused silica [235]. The mass assigned to the imaginary plane is 
equal to the total mass of $\mathrm{Ag}$ atoms in a single (001) atomic plane and the equation of motion is solved for the plane together with the integration of the equations of motion for all the atoms. The parameters of the LJ potential describing the interaction between a $\mathrm{Ag}$ atom and the imaginary plane are fitted to reproduce the experimental value of the work of adhesion between the Ag substrate and silica overlayer as well as the local resistance to uniaxial compression at the interface defined by an arithmetic average of the elastic constant $C_{11}$ for EAM- FBD Ag $\left(C_{11}=124 \mathrm{GPa}\right.$, Ref. [128] $)$ and fused silica $\left(C_{11}=76.6 \mathrm{GPa}\right.$, calculated from experimental values of longitudinal wave velocity and density, Ref. [235]). The work of adhesion for Ag-silica interface at $773 \mathrm{~K}, W_{a d h}=0.43 \mathrm{~J} / \mathrm{m}^{2}$ (Ref. [237] is used to fit the value of the LJ parameter defining the energy scale of the potential, $\varepsilon$. This parameter is chosen so that the energy of the $\mathrm{Ag}$-silica interface, $\gamma_{\mathrm{Ag}^{-} \mathrm{SiO}_{2}}$, satisfies the following definition of the work of adhesion: $W_{a d h}=\gamma_{A g}+\gamma_{S_{i O_{2}}}-\gamma_{A_{g}-S i O_{2}}$, where $\gamma_{A g}=0.72 \mathrm{~J} / \mathrm{m}^{2}$ is the energy of free (001) surface of $\mathrm{Ag}$ calculated at $773 \mathrm{~K}$ for the EAM- FBD potential and $\gamma_{\mathrm{Si}_{2}}=0$ since the energy of the imaginary surface of silica after separation from Ag is zero. The small difference of the value of $\gamma_{A g}$ from the one reported in Ref. [128], $\gamma_{A g}=0.705 \mathrm{~J} / \mathrm{m}^{2}$, can be attributed to different temperature and the cutoff function applied in the current work. The parameter defining the length scale of the potential, $\sigma$, is then chosen to reproduce the local stiffness to uniaxial compression, $C_{11}$, as described above. The fitting yields the following parameters of the LJ potential: $\sigma=2.42 \AA$ and $\varepsilon$ $=0.224 \mathrm{eV}$.

While the representation of the overlayer by the method based on the imaginary plane provides a computationally efficient description of the partial reflection of the laser-induced pressure wave from the metal-silica interface and reproduces the experimental work of adhesion between the metal substrate and silica overlayer, the method is not free of limitations. In particular, the boundary condition can only reproduce the elastic response of the overlayer and no plastic deformation or melting of the overlayer is allowed. Moreover, in the simulations performed under irradiation conditions leading to the transient surface melting and resolidification of the metal target, the imaginary plane is observed to promote nucleation of hcp and fcc crystallites with close-packed crystallographic planes oriented parallel to the imaginary plane. 
In order to relax the assumption of an ideal "indestructible" overlayer, an alternative method, illustrated in Fig. 5.1(b), is developed. In this method, a part of the overlayer adjacent to the metal target is simulated with an atomistic MD method. In the present work, we do not attempt to provide an accurate description of the silica structure and silica-Ag interfacial structure, as such description would necessitate the use of a reactive force field that would substantially increase the computational cost of the simulations. Instead, the silica substrate is simulated as an fcc crystal with atoms interacting through LJ potential. The parameters of the LJ potential $(\sigma=4.41 \AA$ and $\varepsilon=0.237 \mathrm{eV})$ and the atomic mass of $113.5 \mathrm{Da}$ are chosen so that the values of the elastic constant, $C_{11}=75 \mathrm{GPa}$, melting temperature, $T_{m}=1825 \mathrm{~K}$, and density, $\rho_{\mathrm{SiO}_{2}}=2.2 \mathrm{~g} / \mathrm{cm}^{3}$ of the model material are in a good agreement with experimental values for fused silica $\left(C_{11}=76.6 \mathrm{GPa}, T_{m}=1938 \mathrm{~K}, \rho_{\mathrm{SiO}_{2}}=2.2 \mathrm{~g} / \mathrm{cm}^{3}\right.$, Ref. [235]).

The cross-interaction between the atoms in the overlayer and Ag is also described by the $\mathrm{LJ}$ potential. The $\mathrm{LJ}$ parameter $\sigma$ is chosen so that the equilibrium interatomic distance for the cross-interaction would be an arithmetic average of the ones in the substrate and the overlayer. Similarly to the case where the overlayer is represented by an imaginary plane (described above), the LJ parameter $\varepsilon$ is fitted to the work of adhesion for the Ag-silica interface at $773 \mathrm{~K}$, $W_{a d h}=0.43 \mathrm{~J} / \mathrm{m}^{2}$, Ref. [237]. With the calculated surface energies of $\gamma_{A g}=0.72 \mathrm{~J} / \mathrm{m}^{2}$ and $\gamma_{\mathrm{SiO}_{2}}=0.39 \mathrm{~J} / \mathrm{m}^{2}$, the parameter $\varepsilon$ is chosen to reproduce the energy of the Ag-silica interface, $\gamma_{\mathrm{Ag}^{-S i O_{2}}}=\gamma_{\mathrm{Ag}}+\gamma_{\mathrm{SiO}_{2}}-W_{a d h}=0.68 \mathrm{~J} / \mathrm{m}^{2}$. The fitting yields the following parameters of the $\mathrm{LJ}$ potential for the Ag-silica cross-interaction: $\sigma=3.53 \AA, \varepsilon=0.111 \mathrm{eV}$. The size of the part of the overlayer represented with atomistic resolution, $L_{M D}$ in Fig. 5.1(b), is $100 \mathrm{~nm}$ in simulations performed at absorbed laser fluences of 900 and $1000 \mathrm{~J} / \mathrm{m}^{2}$, and $200 \mathrm{~nm}$ at 3000 and $6000 \mathrm{~J} / \mathrm{m}^{2}$. In contrast to the metal substrate, where the heat transfer is dominated by the electrons and is reproduced with the TTM equations, the vibrational heat transfer in the glass overlayer is treated directly by the MD model. The value of the thermal conductivity of the model overlayer material, $k \approx 0.85 \mathrm{Wm}^{-1} \mathrm{~K}^{-1}$, is calculated from the evolution of the temperature profiles predicted in a non-equilibrium MD simulation of a $411 \mathrm{~nm}$ long system with an initial temperature of 300 $\mathrm{K}$ and a temperature of $1000 \mathrm{~K}$ fixed at one end of the system. The conductivity of the model material is comparable to the experimental one for fused silica, $1.38 \mathrm{Wm}^{-1} \mathrm{~K}^{-1}$, Ref. [235]. The 
characteristic length of thermal diffusion in the simulated overlayer on the timescale of the simulation, $t_{\text {sim }}=1 \mathrm{~ns}$, is $L_{t h}=\sqrt{2 D_{T} t_{\text {sim }}} \approx 57 \mathrm{~nm}$, where $D_{T}=k / c_{v} \sim 1.7 \times 10^{-6} \mathrm{~m}^{2} / \mathrm{s}$ is the thermal diffusivity, $k$ is the thermal conductivity, $c_{v} \approx 3 R$ is the heat capacity, and $R$ is the ideal gas constant. Therefore, for $L_{M D}=100-200 \mathrm{~nm}, L_{t h}<L_{M D}$ and the heat transfer through the upper boundary of the computational system in Fig. 5.1(b) is neglected in the simulations. A pressure-transmitting boundary condition $[110,130]$, similar to the one applied at the bottom of the TTM-MD region of the metal target, is applied to the top monolayer of the fcc crystal representing the MD part of the overlayer. The parameters of the boundary condition are chosen to minimize the reflection of the pressure wave propagating through the overlayer from the metal-overlayer interface. Before applying the laser irradiation, all systems are equilibrated at $300 \mathrm{~K}$ for $200 \mathrm{ps}$.

\subsubsection{Result and discussion}

To reveal the effect of the transparent overlayer on laser-induced processes, two series of simulations, with and without the overlayer, are performed for the range of laser fluences that cover the regimes of surface melting, photomechanical spallation, and phase explosion in the absence of the overlayer. The presentation of the results of the simulations is preceded by a brief discussion of the concepts of thermal, stress, and spatial confinement.

\subsubsection{Thermal, stress, and spatial confinement in laser-materials interactions}

Before discussing the effect of the spatial confinement on the material response to the laser excitation, we would like to distinguish it from the thermal and stress confinement realized when the characteristic times of the redistribution of the absorbed laser energy by thermal conduction or stress waves are longer than the time of the temperature increase due to the laser energy deposition $[113,120,121,152,167,238]$. The condition for the thermal confinement, in particular, can be expressed as $\max \left\{\tau_{p}, \tau_{e-p h}\right\} \leq L_{c}^{2} /\left(2 D_{T}\right)$, where $\tau_{p}$ is the laser pulse duration, $\tau_{e-p h}$ is the characteristic time of the electron-phonon equilibration, $L_{c}$ is the diffusive/ballistic penetration depth of the excited electrons before the electron-electron and the electron-phonon equilibration, and $D_{T}$ is the thermal diffusivity of the target material. The condition for the stress 
confinement can be expressed as $\max \left\{\tau_{p}, \tau_{e-p h}\right\} \leq L_{c} / C_{s}$, where $\mathrm{C}_{\mathrm{s}}$ is the speed of sound in the target material.

In the simulations of $\mathrm{Ag}$ targets irradiated by $10 \mathrm{ps}$ laser pulses, $\tau_{p}=10 \mathrm{ps}, \tau_{e-p h} \approx 8 \mathrm{ps}$ (defined as the timescale of the exponential decay of the energy of the excited electrons due to the electron-phonon coupling), $L_{c} \approx 140 \mathrm{~nm}$ (defined as the depth where $63 \%$ of the total excitation energy is deposited by the time $\tau_{e-p h}$ ), $D_{T}=1.47 \times 10^{-4} \mathrm{~m}^{2} / \mathrm{s}$ (near melting temperature), and $C_{s}=3650 \mathrm{~m} / \mathrm{s}$, Ref. [235]), the conditions for both thermal and stress confinement are satisfied and a buildup of both thermal energy and the thermoelastic compressive stresses can be expected in the regions of the laser energy thermalization during the laser pulse $[24,113,120,129,134,152]$. The conditions of the thermal and stress confinement have similar effect on targets with and without the transparent overlayer, thus suggesting that the initial levels of temperature and pressure generated in both cases are likely to be similar.

In contrast to the thermal and stress confinement that define the initial temperature raise and pressure build up in the surface region of the irradiated target, the spatial confinement by the transparent overlayer is affecting the longer-term material response to the laser energy deposition and can have a significant effect on the laser-induced phase transformations, generation of crystal defects, surface damage and material removal (ablation). The results of the atomistic simulations reported below are providing first detailed insights into the kinetics and mechanisms of the structural and phase transformations occurring under conditions of spatial confinement by a transparent overlayer.

\subsubsection{Melting and resolidification under spatial confinement}

The effect of the spatial confinement on the processes of melting and resolidification induced by the laser energy deposition is illustrated in Fig. 5.2, where the temperature and pressure evolution is shown for three simulations performed at the same absorbed laser fluence of $900 \mathrm{~J} / \mathrm{m}^{2}$. 

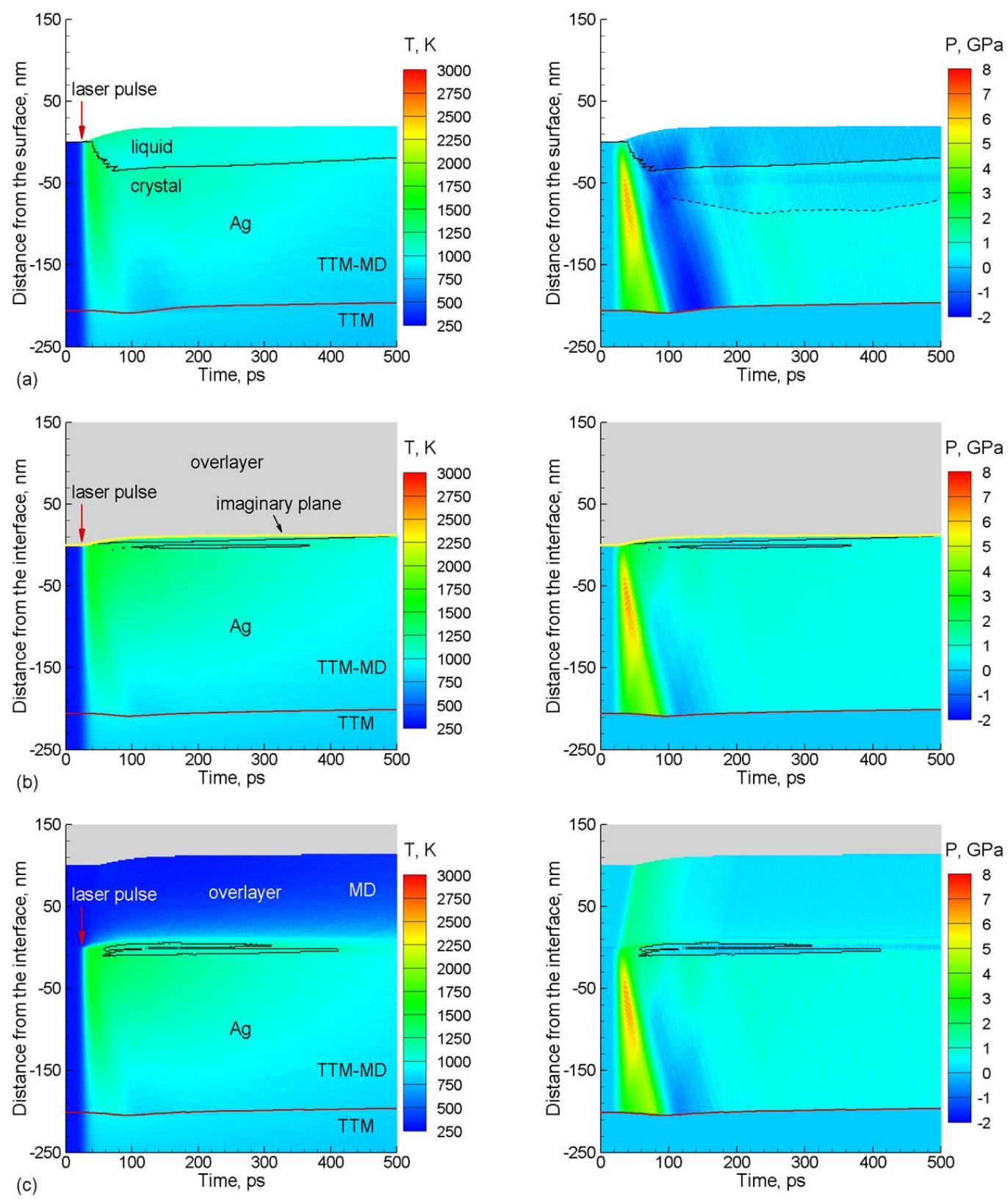

Fig. 5.2. Temperature (left) and pressure (right) contour plots in simulations of a bulk Ag target irradiated by a $10 \mathrm{ps}$ laser pulse at a fluence of $900 \mathrm{~J} / \mathrm{m}^{2}$. The irradiated surface is free of confinement in (a) and is covered by a transparent overlayer in (b,c). The overlayer is represented by a dynamic imaginary plane in (b) and by an atomistic MD description of a part of the overlayer adjacent to the $\mathrm{Ag}$ target combined with a pressure-transmitting boundary condition in (b). The laser pulse has a Gaussian profile with the peak intensity reached at $25 \mathrm{ps}$ from the beginning of the simulations and is directed along the $\mathrm{Y}$-axis, as shown by the red arrows in the left panels. The black curves separate the melted regions from crystalline parts of the Ag target. The red curves separate the atomistic (TTM-MD) and continuum (TTM) parts of the model representing the Ag targets. The dashed curve in the pressure plot in (a) shows the maximum depth of dislocation propagation. The yellow curves in (b) show the position of the Ag-overlayer interface represented by the dynamic imaginary overlayer. 
For a bulk Ag target with a free surface, in the absence of the overlayer, this fluence is about $10 \%$ above the threshold for surface melting. The energy transfer from the excited electrons to the lattice leads to a rapid temperature increase in a surface region of the target, Fig. 5.2(a). The temperature exceeds the equilibrium meting temperature of the EAM Ag, $T_{m}=1139$ $\mathrm{K}$ at zero pressure, and causes a rapid homogeneous melting of $\sim 35 \mathrm{~nm}$ thick surface layer between 40 and 70 ps of the simulation time (15 and 45 ps after the time of the laser peak intensity). The steep temperature gradient established in the surface region by the end of the electron-phonon equilibration leads to the cooling of the melted region and causes epitaxial resolidification. The velocity of the solidification front increases with increasing degree of undercooling below the melting temperature and the front reaches the surface of the target by the time of $\sim 1.1 \mathrm{~ns}$. In the simulations performed for targets covered by an overlayer, Fig. 5.2(b,c), the melting is limited to a few small regions transiently appearing in the vicinity of the interface with the overlayer and disappearing by the time of 400 ps. In the simulation where the overlayer is represented by an imaginary plane, Fig. 5.2(b), the melting is limited to a thin $(<4 \mathrm{~nm})$ layer adjacent to the interface with the overlayer and another region that appears through homogeneous nucleation at a depth of about $10 \mathrm{~nm}$ below the interface. A very similar transient melting is observed in the simulation with the atomistic representation of the overlayer, Fig. 5.2(c), where two small melting regions transiently appear within $20 \mathrm{~nm}$ from the interface with the overlayer. The difference of these results from the much more extensive melting observed in the absence of the overlayer, Fig. 5.2(a), is surprising from the first sight, as the same initial temperature increase is caused by the laser energy deposition and the temperature decrease is even faster due to the free expansion of the surface region. To explain this difference, the evolution of the laser-induced pressure is considered below.

As discussed in Section 5.1.3.1, the rapid increase of the lattice temperature in the simulations takes place under conditions of the stress confinement and results in the buildup of strong compressive stresses in the surface region of the irradiated target. The generation of compressive stresses of similar strength can be seen in all pressure plots shown in Fig. 5.2. The relaxation of the compressive stresses, however, proceeds differently with and without the confining overlayer. Without the overlayer, the expansion of the compressed material results in the generation of a strong tensile component of the stress wave that follows the compressive component in its propagation into the bulk of the target, Fig. 5.2(a). In the presence of the 
overlayer, however, the tensile component of the stress wave is largely suppressed, Fig. 5.2(b,c). Since the acoustic impedance of the overlayer material is more than twice smaller than the one of $\mathrm{Ag}$, the relaxation of the initial laser-induced compressive stresses results the generation of both a compressive wave in the overlayer and a relatively weak unloading wave in the Ag target

The suppression of the tensile component of the pressure wave by the overlayer has direct implications for the melting process. In the absence of the overlayer, the expansion of the surface region results in the tensile stresses and, concurrently, a transient temperature drop that can be estimated by considering the expansion as an isentropic process, for which $(\partial T / \partial P)_{S}=T \alpha / c_{P}$, $[118,121]$ where $c_{p}$ is the specific heat capacity per unit volume and $\alpha$ is the volume coefficient of thermal expansion. At the same time, the equilibrium melting temperature is also affected by the negative stresses, with the variation of the melting temperature described by the Clapeyron equation, $(d T / d P)_{m} \approx \Delta V_{m} / \Delta S_{m}$, where $\Delta V_{m}$ and $\Delta S_{m}$ are the volume change upon melting and the entropy of melting, respectively. Following an approach described in Ref. [118], the thermodynamic parameters of the FBD EAM Ag, $\Delta V_{m}=0.81 \mathrm{~cm}^{3} / \mathrm{mol}, \Delta H_{m}=12350 \mathrm{~J} / \mathrm{mol}, \Delta S_{m}$ $=\Delta H_{m} / T_{m}=10.84 \mathrm{~J} / \mathrm{mol} \mathrm{K}$, as well as $\alpha=102 \times 10^{-6} \mathrm{~K}^{-1}$ and $c_{p}=31.8 \mathrm{~J} / \mathrm{mol} \mathrm{K}=2.85 \mathrm{~J} / \mathrm{cm}^{3} \mathrm{~K}$ for the solid phase at $T \approx T_{m}$ and zero pressure, have been evaluated based on the temperature dependences of volume and internal energy of liquid and solid phases at constant pressure. With these thermodynamic parameters, $(d T / d P)_{m} \approx 74 \mathrm{~K} / \mathrm{GPa}$ and $(\partial T / \partial P)_{S} \approx 41 \mathrm{~K} / \mathrm{GPa}$ for the solid phase close to the melting temperature and zero pressure. The steeper slope of the liquid-crystal coexistence line compared to the isentrope for the solid phase, $(d T / d P)_{m}>(\partial T / \partial P)_{s}$, typical for metals, suggests that the expansion of the target by the unloading wave facilitates melting despite simultaneously causing a transient cooling of the material. This explains why the partial suppression of the generation of the unloading wave also suppresses melting in the simulations of Ag targets covered by overlayers.

An examination of the pressure contour plots in Fig. 5.2 also reveals that, in all simulations, the propagation of the unloading wave takes place on a background quasi-static compressive stresses related to the inability of the crystalline part of the target to completely relax the thermoelastic stresses through uniaxial expansion in the direction normal to the irradiated surface or metal-overlayer interface $[120,129,152]$. The compressive stresses are partially relaxed by the emission of dislocations from the melting front in the simulation with 
free surface, Fig. 5.2(a), where the depth of the dislocation propagation can be seen from the reduction of the compressive stresses in a region extending down to $\sim 45 \mathrm{~nm}$ below the melting front. The emission of the dislocations coincides with the propagation of the unloading wave through the melting front and is triggered by the shear stresses transiently created by the unloading wave in the four different active $\{111\}$ slip planes of the single crystal fcc target oriented along <100> direction [121]. Interactions between the Shockley partial dislocations propagating on different slip planes result in the generation of immobile dislocation segments and formation of stable dislocation configurations in the sub-surface region of the target. The much weaker unloading wave generated in the presence of the overlayer is incapable of inducing the dislocation emission and no dislocations are observed in simulations illustrated in Fig. $5.2(b, c)$.

The results obtained with two different representations of the overlayer, Fig. 5.2(b,c), are very similar to each other. This similarity suggests that both approaches provide an adequate description of the effect of the overlayer on the target response to the laser excitation under irradiation conditions that leave the overlayer intact. At higher laser fluences, however, a part of the overlayer adjacent to the metal target can be expected to undergo transient melting and possible mixing with the melted metal. To provide a semi-quantitative description of these processes, the atomistic representation of a part of the overlayer adjacent to the metal target (Fig. 5.1(b)) is used in the higher-fluence simulations presented below.

\subsubsection{Suppression of laser spallation by the overlayer}

A moderate increase in the absorbed laser fluence from $900 \mathrm{~J} / \mathrm{m}^{2}$ to $1000 \mathrm{~J} / \mathrm{m}^{2}$ leads, in the absence of overlayer, to the transition from the regime of melting and resolidification of a surface region of the target to a separation of a layer of melted material from the bulk of the target, Fig. 5.3(a). As discussed in a number of earlier papers, e.g. Refs. [24, 113, 120, 134, 152], the separation and ejection of the liquid layer (commonly referred to as spallation) is driven by the relaxation of the laser-induced stresses generated in the surface region of the target under conditions of stress confinement. 

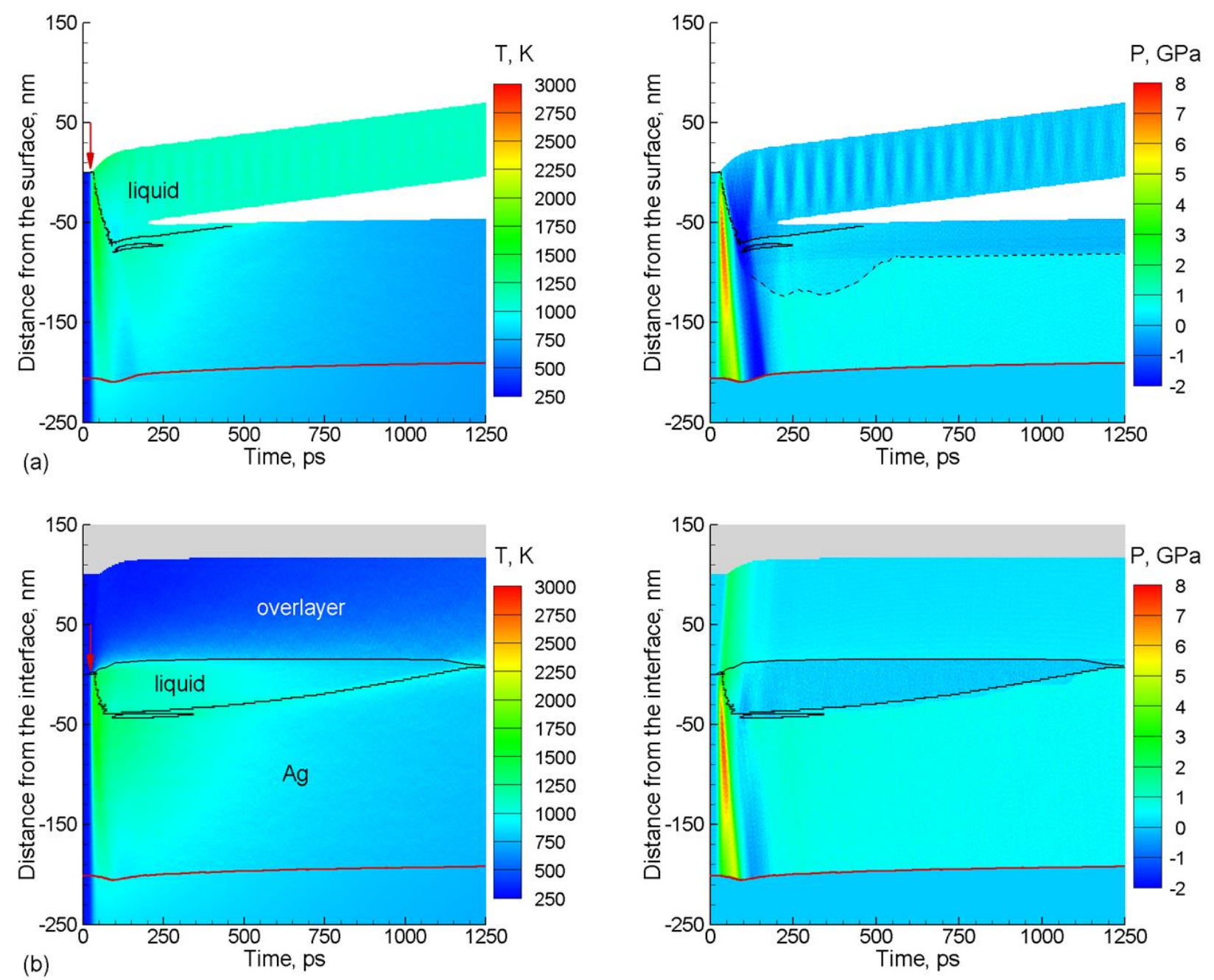

Fig. 5.3. Temperature (left) and pressure (right) contour plots in simulations of a bulk Ag target irradiated by a $10 \mathrm{ps}$ laser pulse at a fluence of $1000 \mathrm{~J} / \mathrm{m}^{2}$. The irradiated surface is free of confinement in (a) and is covered by a transparent overlayer in (b). The computational setup in the simulation with overlayer is illustrated in Fig. 5.1(b). The laser pulse has a Gaussian profile with the peak intensity reached at 25 ps from the beginning of the simulations and is directed along the Y-axis, as shown by the red arrows in the left panels. The black curves separate the melted regions from crystalline parts of the Ag target. The red curves separate the atomistic (TTM-MD) and continuum (TTM) parts of the model representing the $\mathrm{Ag}$ targets. The dashed curve in the pressure plot in (a) shows the maximum depth of dislocation propagation. Areas where the density of the material drops below $10 \%$ of the initial density of the solid Ag target are blanked in the plots. 
The magnitude of both the compressive and tensile stresses increases with increasing fluence up to the spallation threshold, when the tensile stress exceeds the dynamic strength of the melted material leading to a nucleation, growth, and coalescence of multiple voids that eventually causes spallation of a liquid layer from the target. The spallation interrupts the heat flow from the hot surface layer to the bulk of the target and results in a rapid resolidification of the liquid part of the target left behind after the separation of the spalled layer. In the simulation illustrated in Fig. 5.3(a), the resolidification process is completed at $\sim 50$ ps after the laser pulse. Similarly to the lower fluence simulation discussed in Section 5.1.3.2, the emission of dislocations from the melting front takes place at the time when the tensile component of the stress wave crosses the melting front and relaxes the residual compressive stresses in $\sim 40 \mathrm{~nm}$ surface region of the resolidified target.

Turning to the simulation of a Ag target covered by an overlayer, Fig. 5.3(b), the following effects of the spatial confinement can be observed: (1) the depth of the region affected by melting decreases from $\sim 70 \mathrm{~nm}$ in Fig. 5.3(a) to $\sim 40 \mathrm{~nm}$ in Fig. 5.3(b), (2) no dislocation emission from the melting front takes place, and (3) no voids nucleate in the melted region and no spallation occurs. All of these effects can be attributed to the containment of the free expansion of the surface region of the target and the corresponding suppression of the tensile component of the stress wave.

The suppression of the tensile stresses is illustrated in Fig. 5.4, where the evolution of pressure at a depth of $100 \mathrm{~nm}$ below the irradiated Ag surface is shown in the form of pressure profiles. Although the initial peaks of the compressive pressure are almost identical in the two simulations, with the maximum pressure reaching $\sim 6.7 \mathrm{GPa}$ at $\sim 45 \mathrm{ps}$ after the laser pulse, the pressure profiles diverge after $\sim 70$ ps. In the case of free surface, the unloading tensile component of the pressure wave, while weakened by the void nucleation and spallation in the region closer to the surface, is still clearly pronounced and reaches the maximum value of -2 $\mathrm{GPa}$. In the presence of the overlayer, however, the pressure at this depth remains positive at all times. Following the initial dynamic relaxation of the laser-induced stresses, after $\sim 250 \mathrm{ps,} \mathrm{the}$ level of pressure stays at an almost constant level that is slowly drifting from $\sim 0.8$ to $0.6 \mathrm{GPa}$ during the time from 250 to 700 ps. As discussed in Section 5.1.3.2, this compressive pressure corresponds to the residual thermoelastic stresses that cannot relax by the uniaxial expansion of the crystal in the direction normal to the surface and are slowly decreasing due to the cooling by 
thermal conduction to the bulk of the target. Note that the level of the residual stresses in the simulation with free surface stays at a lower level of $\sim 0.2-0.3 \mathrm{GPa}$ until the time of $\sim 530 \mathrm{ps}$, when it increases to the level similar to the one observed in the presence of the overlayer. The reduction of the compressive stresses can be attributed to dislocations that propagate deeper than $100 \mathrm{~nm}$ during the initial relaxation of laser-induced stresses and bring the stress state of the crystal closer to hydrostatic one. The partial dislocations retreat up to the depth of $\sim 80 \mathrm{~nm}$ below the original surface during the time of $400-600 \mathrm{ps}$ and the increase of the level of pressure at around $530 \mathrm{ps}$ corresponds to the time when they cross the depth of $100 \mathrm{~nm}$.

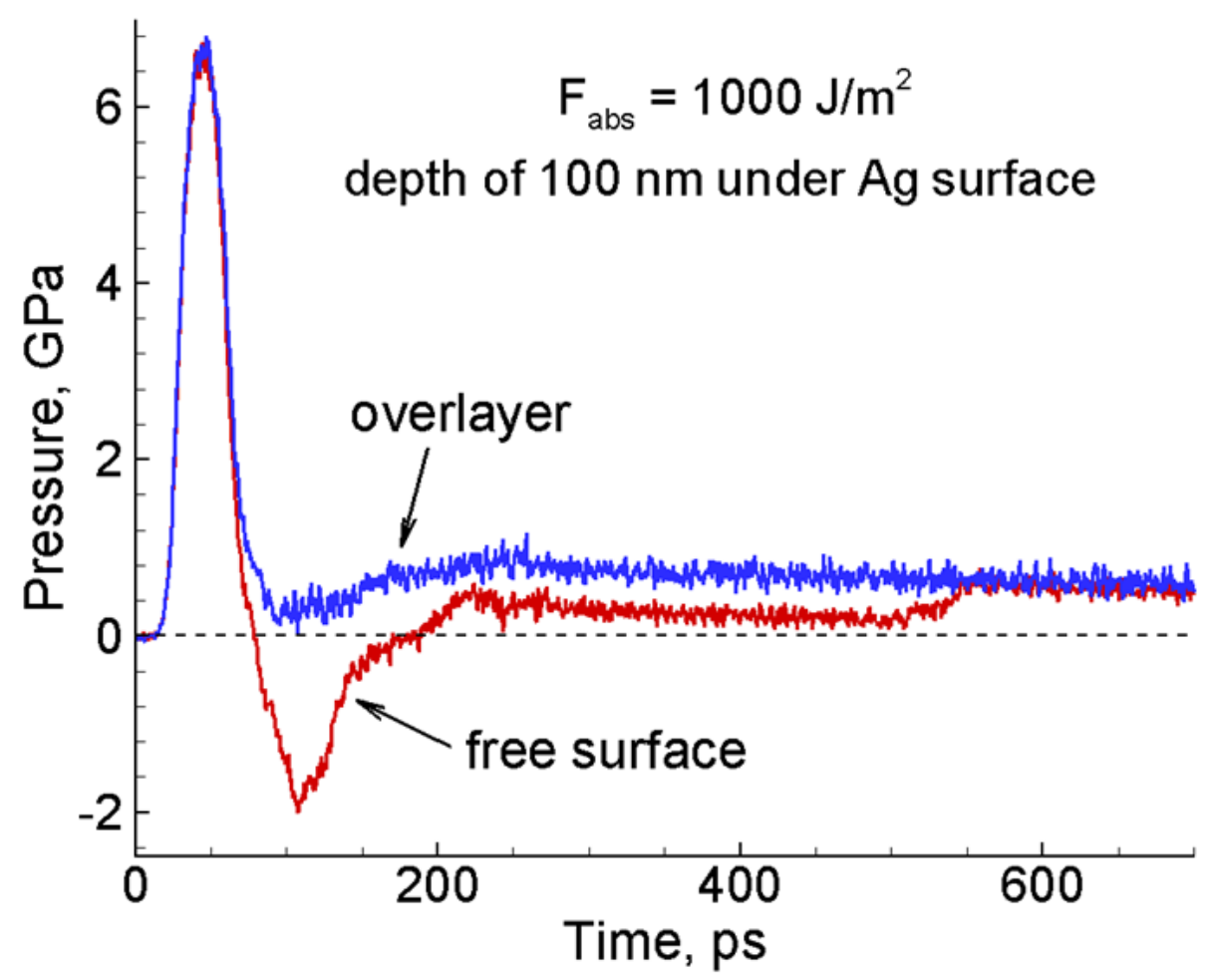

Fig. 5.4. Pressure profiles at a depth of $100 \mathrm{~nm}$ below the surface of Ag targets in simulations performed with and without the transparent overlayer and illustrated by contour plots in Fig. 5.3. 
In the absence of spallation, the resolidification process in the target covered by an overlayer takes about $1.25 \mathrm{~ns}$, Fig. 5.3(b), the time comparable to that in the simulation of a target with free surface performed at a lower fluence of $900 \mathrm{~J} / \mathrm{m}^{2}$, below the spallation threshold,

Fig. 5.2(a). The melting turns into resolidification when the temperature of the liquid-solid interface drops below the melting temperature at $\sim 150 \mathrm{ps}$, the solidification front accelerates with increasing supercooling and reaches its maximum velocity of $\sim 65 \mathrm{~m} / \mathrm{s}$ by the time of $1.1 \mathrm{~ns}$. By this time, however, the temperature of Ag near the interface with the overlayer drops down to $\sim 0.7 T_{m}$, triggering nucleation of new crystallites near the interface and leading to the rapid solidification of the strongly supercooled layer of Ag adjacent to the interface. The relatively small lateral size of the computational cell used in the simulations does not allow for a reliable investigation of the nucleation and growth of new crystallites in the supercooled interfacial region of the target and new larger scale simulations targeted at analysis of this phenomenon are currently underway.

\subsubsection{Suppression of phase explosion by the overlayer}

As the laser fluence increases above the spallation threshold for targets with free surfaces, the thickness of the spalled layer is decreasing while the temperature and the ejection velocity are increasing [24]. The spalled layer vanishes close to the fluence that brings the temperature of the surface region of the target above $T^{*}=3450 \mathrm{~K}$, identified in Section 5.1.2.1 as the temperature of the onset of the explosive phase separation into liquid and vapor at zero pressure. This signifies a transition to the phase explosion regime of material ejection, when the surface region undergoes an explosive decomposition into vapor, clusters and small droplets.

The contour plots shown in Fig. 5.5(a) are for a simulation performed for a fluence of $3000 \mathrm{~J} / \mathrm{m}^{2}$, slightly above the threshold for the transition from spallation to the phase explosion regimes of laser ablation. The temperature plot exhibits a momentary (between 30 to $60 \mathrm{ps}$ of the simulation or 5 to $35 \mathrm{ps}$ after the peak of the $10 \mathrm{ps}$ laser pulse) spike to the values exceeding $T^{*}$ at zero pressure in the surface region with a depth of $\sim 115 \mathrm{~nm}$, close to depth of the initial laser energy deposition $L_{c}$, see Section 5.1.3.1. 

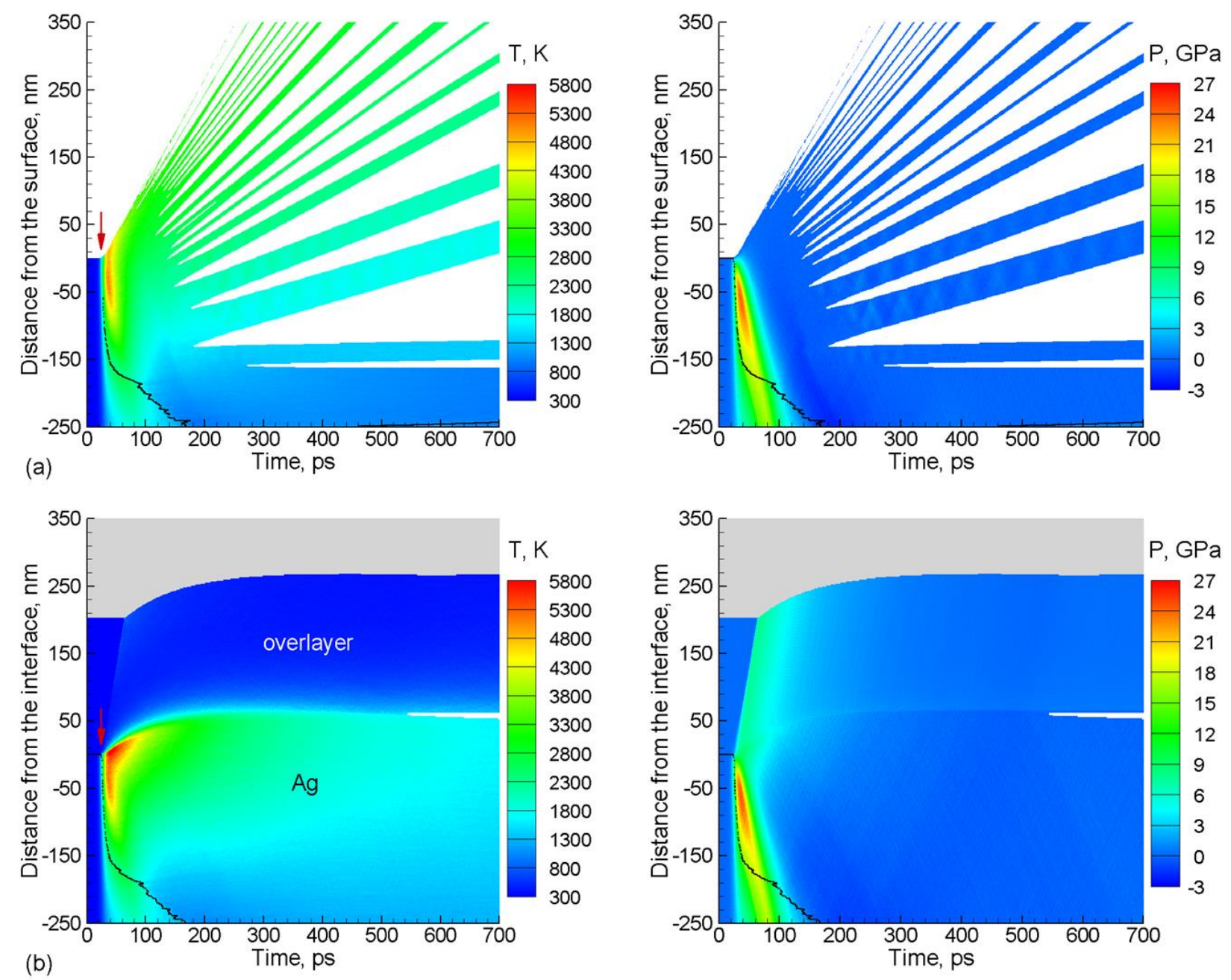

Fig. 5.5. Temperature (left) and pressure (right) contour plots in simulations of a bulk Ag target irradiated by a $10 \mathrm{ps}$ laser pulse at a fluence of $3000 \mathrm{~J} / \mathrm{m}^{2}$. The irradiated surface is free of confinement in (a) and is covered by a transparent overlayer in (b). The computational setup in the simulation with overlayer is illustrated in Fig. 5.1(b). The laser pulse has a Gaussian profile with the peak intensity reached at 25 ps from the beginning of the simulations and is directed along the $\mathrm{Y}$-axis, as shown by the red arrows in the left panels. The black curves separate the melted regions from crystalline parts of the $\mathrm{Ag}$ target. Areas where the density of the material drops below $10 \%$ of the initial density of the solid $\mathrm{Ag}$ target are blanked in the plots. 
The rapid cooling of this region proceeds through the expansion and decomposition of the material overheated above the limit of its thermodynamic stability into a mixture of liquid droplets, atomic clusters and vapor. The unloading wave, while partially suppressed by the compressive pressure generated by the explosive decomposition and expansion of the top layer of the target, still generates tensile stresses that exceed -1 GPa below the depth of $\sim 140 \mathrm{~nm}$. These tensile stresses induce cavitation of the superheated liquid deeper into the target and, similarly to the spallation discussed above, lead to the ejection of additional large droplets.

In contrast to the explosive decomposition of a surface region discussed above, no phase separation is observed in a simulation performed at the same laser fluence for a target covered by an overlayer, Fig. 5.5(b). The initial temperature increase is similar to the one in a target with free surface and brings the surface region to a supercritical state. Although the pressure from the hot metal pushes the overlayer up by $65 \mathrm{~nm}$, the supercritical fluid remains confined by the overlayer. This confinement prevents the phase decomposition and fast cooling observed for a target with free surface and keeps the temperature and pressure of the surface region at high levels for a much longer time. Only by the time of $350 \mathrm{ps}$ the pressure in the interfacial regions drops down to zero and the expansion of the metal target turns into contraction as the interfacial region continues to cool down by thermal conduction to the bulk of the target. The contraction results in the formation of voids near the interface and eventual detachment of the metal target from the overlayer. In the absence of the phase explosion, which provides an efficient channel of fast cooling of the hot surface region of the target in the absence of overlayer, the maximum melting depth is larger (270 nm vs $250 \mathrm{~nm}$ in the simulation with free surface) and the time needed for resolidification of the metal target is much longer. The emission of partial dislocations, though, is observed at this fluence both with and without overlayer.

An additional illustration of the effect of spatial confinement by the overlayer on the conditions experienced by the top layer of the metal target is provided in Fig. 5.6, where the temperature-pressure trajectories are shown for material initially located within the top $5 \mathrm{~nm}$ layer of the target. In the simulation with free surface, the initial linear pressure-temperature dependence characteristic of almost constant volume heating of the layer (stress confinement) gives way to the pressure drop associated with the onset of free expansion of the top layer at $\sim 25$ ps. The relaxation of the laser-induced pressure and continued temperature increase makes the trajectory cross the solid curve that marks the temperature and pressure conditions for the onset 
of the explosive phase separation into liquid and vapor, $T^{*}$ and $P^{*}$, determined as explained in Section 5.1.2.1. In the presence of overlayer, however, the expansion of the hot metal layer is limited and the pressure builds up to much higher levels. The expansion of the surface allowed by up to $65 \mathrm{~nm}$ upward displacement of the overlayer results in a pressure plateau at the end of the electron-phonon energy equilibration and leads to a hysteresis in the pressure-temperature dependence, when the pressure decrease during the cooling of the interfacial region along the path that is almost parallel to the one of the heating stage but shifted to lower values of pressure for the same temperature. This hysteresis eventually leads to the detachment of the metal target from the overlayer when the pressure near the interface becomes negative after about 400 ps, when the temperature drops below $1800 \mathrm{~K}$.

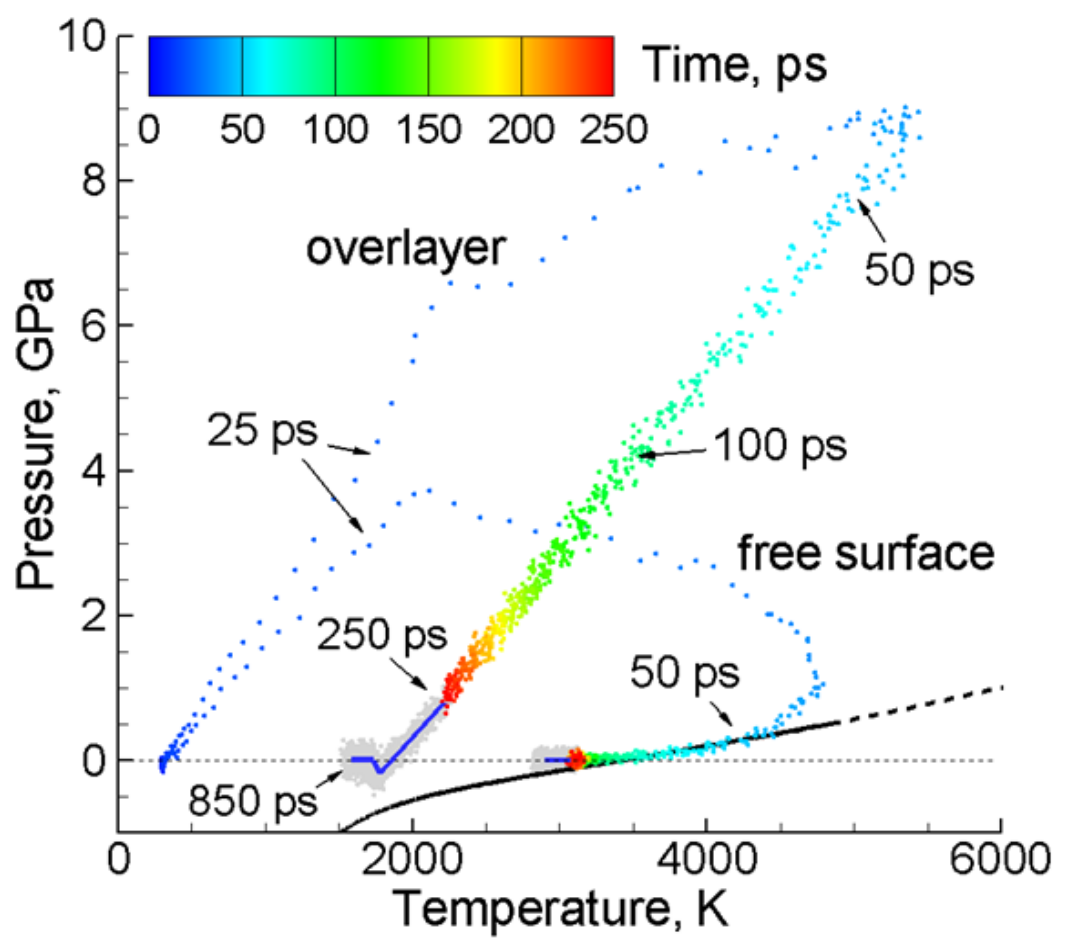

Fig. 5.6. The evolution of temperature and pressure averaged over atoms that are initially located in the top $5 \mathrm{~nm}$ thick layer of the $\mathrm{Ag}$ target irradiated by $10 \mathrm{ps}$ laser pulses at a fluence of $3000 \mathrm{~J} / \mathrm{m}^{2}$ with and without the presence of a transparent overlayer. The points along the trajectories are colored by time up to $250 \mathrm{ps}$ as shown by the legend; from 250 to $850 \mathrm{ps}$ the points are gray with blue lines drawn as a guide to the eye. The black curve shows the temperature-pressure conditions $\left(\mathrm{T}^{*}, \mathrm{P}^{*}\right)$ for the onset of the cavitation or phase explosion in the metastable liquid, calculated for the EAM Ag material as explained in Section. 5.1.2.1. 
The overall picture of the material response to the laser energy deposition with and without the overlayer does not change even when the laser fluence is doubled from $3000 \mathrm{~J} / \mathrm{m}^{2}$ to $6000 \mathrm{~J} / \mathrm{m}^{2}$, Figs. 5.7 and 5.8. The much stronger laser excitation leads to higher maximum temperature and pressure, larger displacement of the overlayer, and higher melting depths of 455 and $415 \mathrm{~nm}$ with and without overlayer, respectively. The combination of the phase explosion and material expulsion due to the unloading wave leads to almost complete ejection of melted part of the target down to the depth of $385 \mathrm{~nm}$ in the case of the target with a free surface, Fig. 5.7(a). In the presence of the overlayer, the expansion of the metal brought to the supercritical state by the fast laser energy deposition is limited by the overlayer that maintains the high density and pressure of the supercritical fluid for hundreds of ps and prevents its decomposition into vapor and liquid droplets. The pressure exerted by the supercritical Ag pushes the overlayer by up to $137 \mathrm{~nm}$ by the time of $600 \mathrm{ps,}$, when the pressure approaches zero level, Fig. 5.7(b). Similarly to the lower fluence simulation illustrated in Fig. 5.5(b), the cooling and contraction of the metal target leads to its detachment from the overlayer at around 800 ps, Fig. 5.7(b). The temperature of the metal near the interface is about $2500 \mathrm{~K}$, more than twice above the melting temperature but below the stability limit for phase explosion. Due to the high temperature of the interfacial region, the detachment takes place at a relatively low negative pressure of about -0.11 $\mathrm{GPa}$ (this value is obtained by averaging from 700 to $800 \mathrm{ps}$ over the top $50 \mathrm{~nm}$ part of the metal target). 

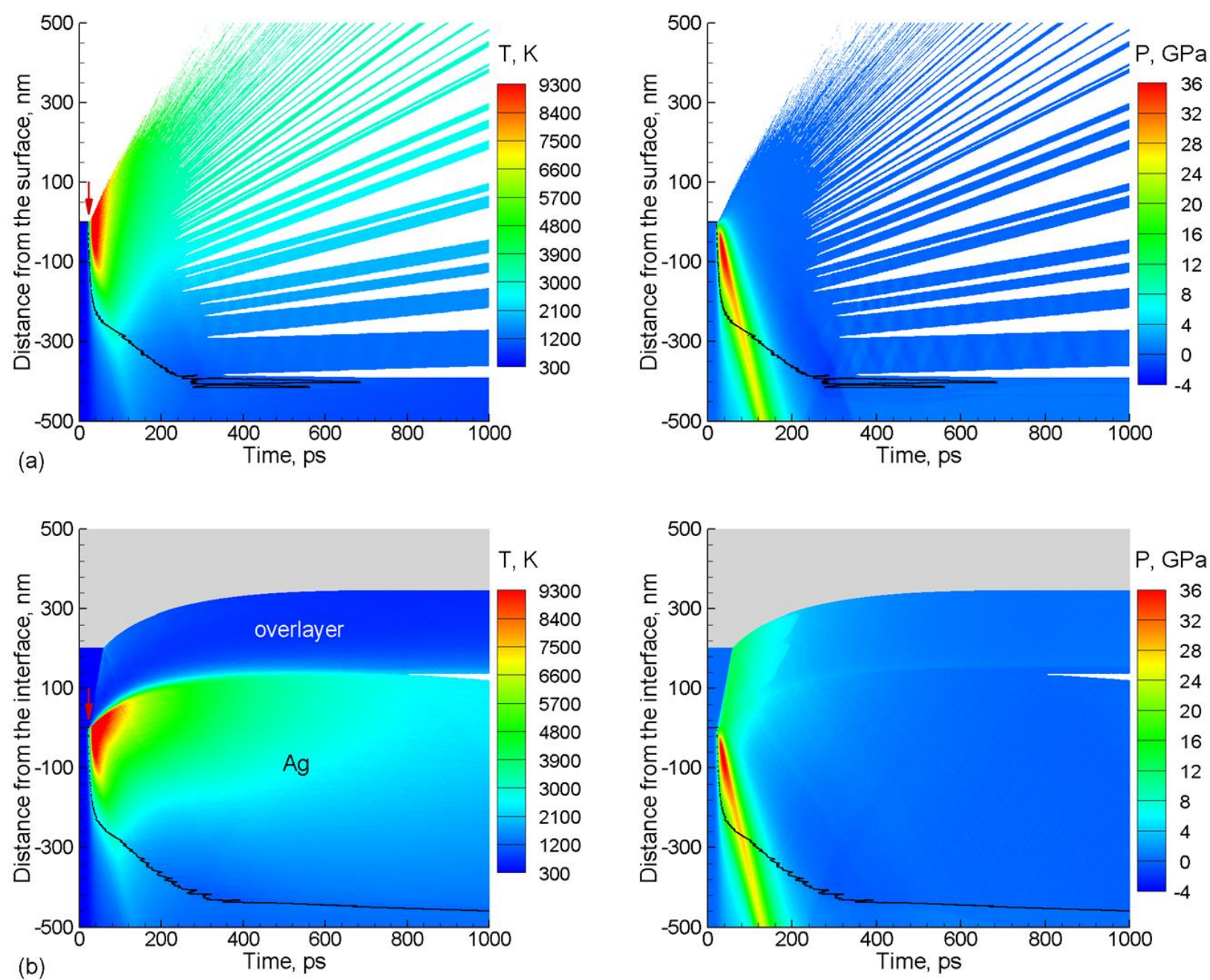

Fig. 5.7. Temperature (left) and pressure (right) contour plots in simulations of a bulk Ag target irradiated by a $10 \mathrm{ps}$ laser pulse at a fluence of $6000 \mathrm{~J} / \mathrm{m}^{2}$. The irradiated surface is free of confinement in (a) and is covered by a transparent overlayer in (b). The computational setup in the simulation with overlayer is illustrated in Fig. 5.1(b). The laser pulse has a Gaussian profile with the peak intensity reached at $25 \mathrm{ps}$ from the beginning of the simulations and is directed along the Y-axis, as shown by the red arrows in the left panels. The black curves separate the melted regions from crystalline parts of the Ag target. Areas where the density of the material drops below $10 \%$ of the initial density of the solid Ag target are blanked in the plots. 
The temperature-pressure trajectories shown in Fig. 5.8 further illustrate the difference in thermodynamic conditions experienced by the material in the top surface layers of the two targets. While in both simulations the strong laser excitation brings the layers into supercritical state, the evolution of the supercritical fluid is very different in the two cases. In the absence of overlayer, free expansion of the supercritical fluid releases the pressure and result in complete vaporization of the material. The supercritical fluid confined by the overlayer, on the other hand, slowly cools down from the supercritical to the liquid state without crossing the line that corresponds to the explosive phase separation.

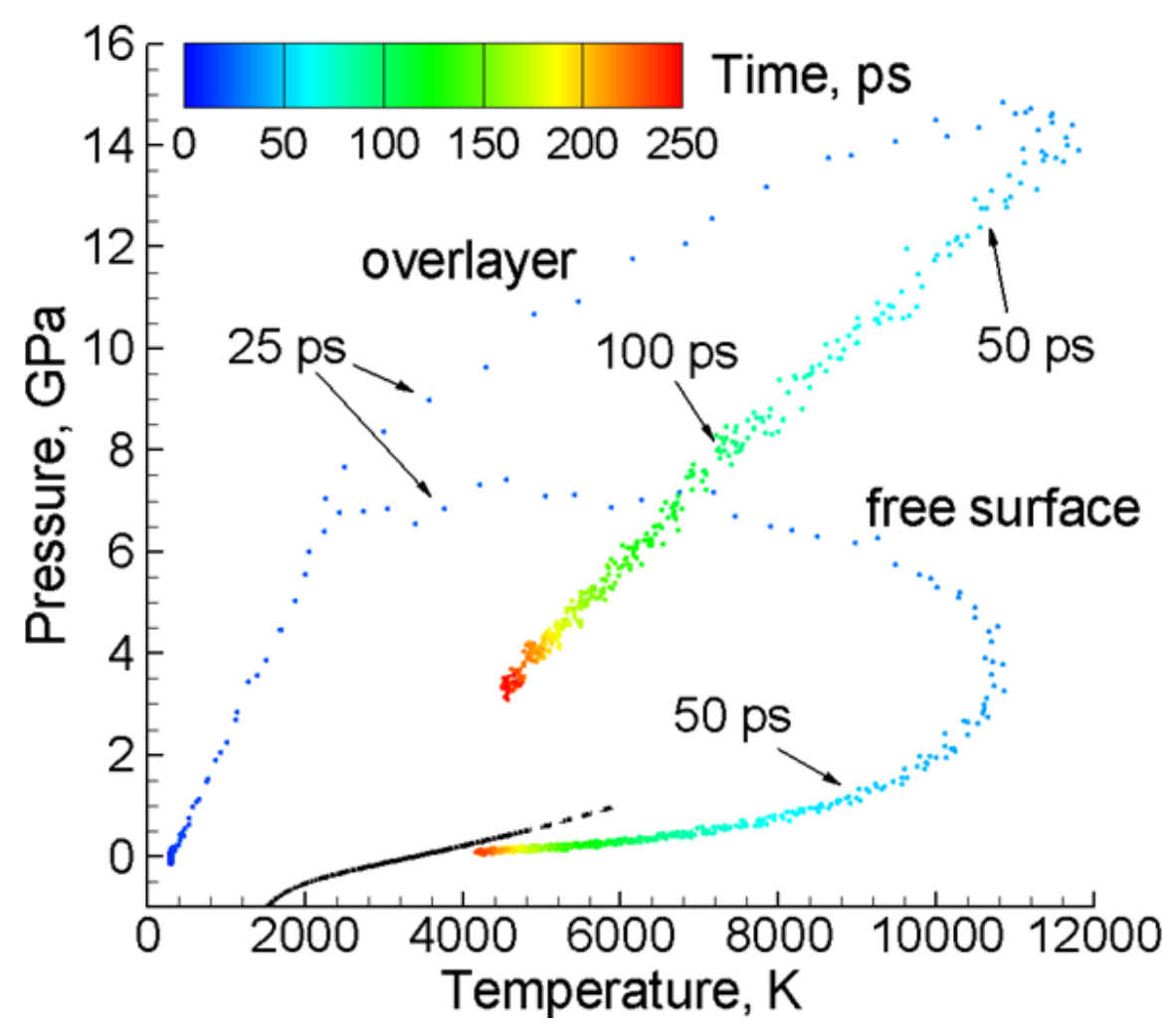

Fig. 5.8. The evolution of temperature and pressure averaged over atoms that are initially located in the top $5 \mathrm{~nm}$ thick layer of the Ag target irradiated by $10 \mathrm{ps}$ laser pulses at a fluence of $6000 \mathrm{~J} / \mathrm{m}^{2}$ with and without the presence of a transparent overlayer. The points along the trajectories are colored by time as shown by the legend. The black curve shows the temperature-pressure conditions $\left(\mathrm{T}^{*}, \mathrm{P}^{*}\right)$ for the onset of the cavitation or phase explosion in the metastable liquid, calculated for the EAM Ag material as explained in Section. 5.1.2.1. 
The observation that the maximum temperature of the top region reaches $\sim 12,000 \mathrm{~K}$ in the simulation with overlayer and $\sim 11,000 \mathrm{~K}$ without overlayer by the time of $\sim 40$ ps suggests the possibility of ionization of a fraction of $\mathrm{Ag}$ atoms. Indeed, estimations based on the Saha equation yields ionization fraction of about $1 \%$ at densities realized during the peak temperatures. The short time of the temperature spike (the temperature drops down to $9000 \mathrm{~K}$ by $80 \mathrm{ps}$ and down to $6000 \mathrm{~K}$ by $170 \mathrm{ps}$ in the simulation with overlayer, where the cooling is slower) and the high density of the supercritical fluid indicates, however, that any effects related to the ionization and charge fluctuations [23] in the supercritical fluid are likely to have negligible effect on the material expansion and interaction with the overlayer under conditions considered in the simulations.

The exposure of the overlayer to the hot metal leads to the melting of a part of the overlayer adjacent to the metal target, with the thickness of the melted layer reaching $15 \mathrm{~nm}$ by the time of the detachment from the target. The interaction of the supercritical Ag with melted overlayer results in an active atomic mixing in the interfacial region. The mixing is illustrated in Fig. 5.9, where the atomic configurations and concentration profiles in the vicinity of the metaloverlayer interface are shown for $780 \mathrm{ps}$, just before the detachment of the metal from the overlayer. The observation that more atoms of the overlayer material penetrate into the metal can be explained by much higher temperatures of the metal and a relatively weak interaction between the overlayer and Ag atoms. The Ag atoms are also diffusing into the melted part of the overlayer and tend to aggregate into clusters due to the stronger interactions between the metal atoms as compared to the metal-overlayer interactions. Note that the approximate representation of the overlayer by atoms interacting with each other through pairwise LJ potential prevents a quantitative analysis of the atomic mixing and formation of new phases in the interfacial region. At a qualitative level, however, we can conclude that a substantial interfacial mixing can be expected to result in a high concentration of impurities, atomic clusters and/or new phases in both the overlayer and metal sides of the interface. A quantitative analysis of the nanostructure of the interfacial region can be performed by adopting the computational setup in Fig. 5.1(b) for simulations with a realistic representation of interatomic interactions accounting for the mixed covalent-metallic nature of bonding in the interfacial region. 


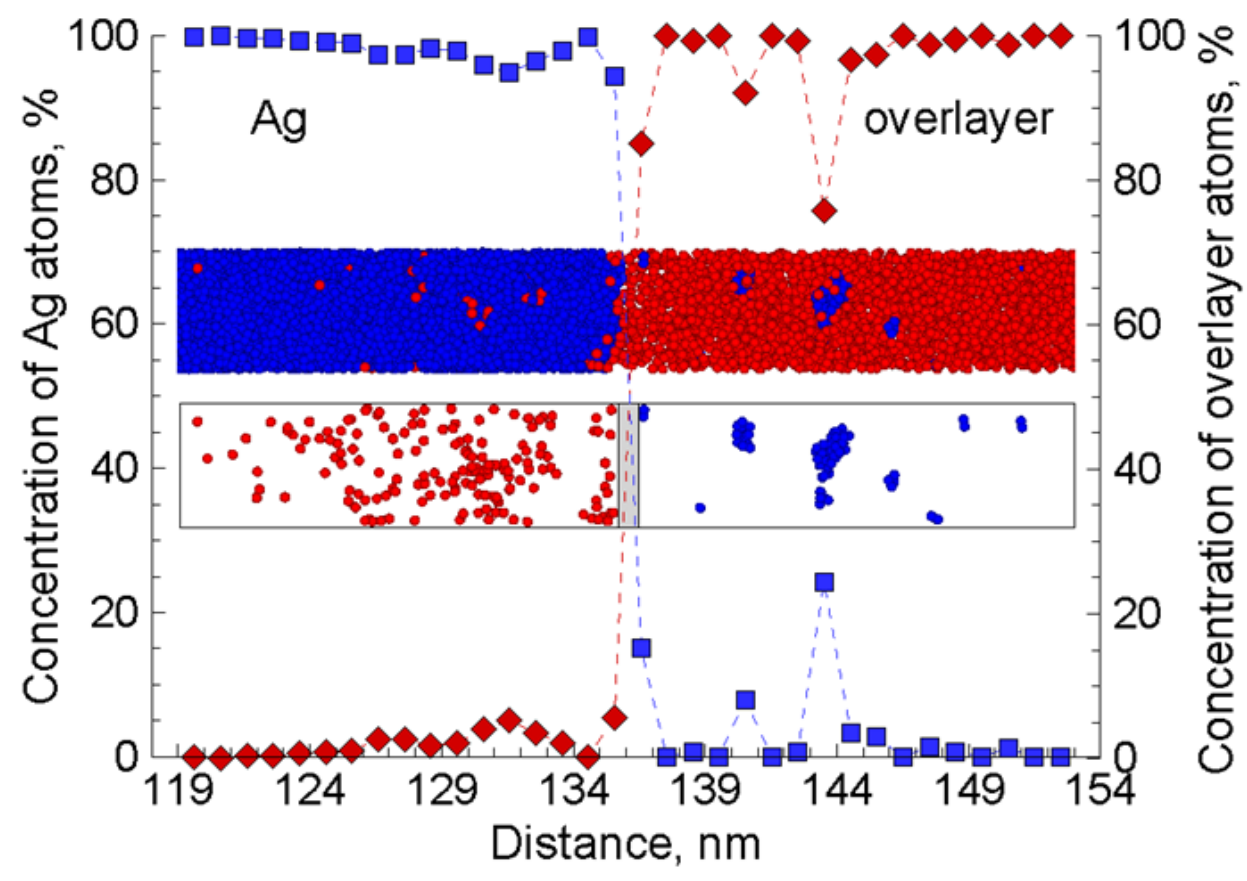

Fig. 5.9. Concentration profiles and atomic configuration in the region of Ag-overlayer interface at 780 ps after irradiation by a $10 \mathrm{ps}$ laser pulse at a fluence of $6000 \mathrm{~J} / \mathrm{m}^{2}$. The atoms in the snapshot are colored by their type, with blue and red colors used for Ag and overlayer atoms, respectively. In the lower version of the snapshot, the Ag atoms are blanked on the left side of the interface and the overlayer atoms are blanked on the right side of the interface to expose the atomic mixing.

\subsubsection{Summary}

The effect of spatial confinement on laser-induced structural and phase transformation in a metal target is investigated by contrasting the results of two series of simulations of short pulse laser interactions with a bulk Ag target. The metal target is covered by a thick transparent overlayer in one series of simulations and has a free surface in another series. The simulations are performed with a combined atomistic-continuum TTM-MD model accounting for the laser excitation of conduction band electrons, electron-phonon equilibration, as well as electronic and phononic heat transfer in the metal target and the overlayer. The model is extended by incorporation of two alternative computational methods for representations of the transparent overlayer. In one method, the layer is represented by a dynamic acoustic impedance matching boundary condition designed to represent the partial propagation of the laser-induced pressure 
wave into the overlayer, as well as work of adhesion between the metal and the overlayer. In the second method, a part of the overlayer is represented with atomic resolution, allowing not only to account for the elastic response of the overlayer but also to allow for plastic deformation, melting, and atomic mixing in the region of the overlayer adjacent to the hot metal target. Both methods are parameterized for Ag-silica system and show similar performance at low fluences, when no melting of the overlayer takes place. The second (atomistic) representation of the metaloverlayer interface is used in the simulations performed at high fluences, when melting and atomic mixing takes place on both sides of the interface.

The simulations are performed for a broad range of laser fluences that, in the absence of overlayer, covers the regimes of melting and resolidification, photomechanical spallation, and phase explosion of the surface region of the metal target. At low fluences that correspond to melting and spallation of targets with free surfaces, the spatial confinement by the overlayer is found suppress the generation of the unloading tensile wave, decrease the maximum depth of melting, reduce or eliminate the emission of dislocations from the melting front, and prevent the cavitation and spallation in the surface region of the metal target.

At higher fluences that bring the surface region of the metal target to the supercritical state, the confinement prevents the expansion and explosive phase decomposition of the surface region and leads to a gradual cooling of the hot and compressed supercritical fluid down to the liquid phase. This is in a sharp contrast with the material response to the same laser energy deposition under the vacuum conditions (free surface of the target), when the free expansion of the supercritical fluid results in cooling and phase decomposition into liquid droplets and vapor or complete vaporization of the top surface layer at higher fluences. The confinement of the superheated metal by the overlayer increases the maximum depth of melting and the time required for resolidification after the laser pulse. Moreover, the relatively long lifetime of the supercritical state sustained under conditions of spatial confinement results in a transient melting of a part of the overlayer exposed to the hot metal and atomic mixing between the overlayer material and metal within tens of nanometers from the interface. The simulations also suggest possibility of detachment of the metal target from the overlayer during the rapid cooling and contraction of the surface region of the metal target. A detailed analysis of the evolution of voids leading to the detachment at the interface as well as the possible formation of a thin multiphase 
nanocrystalline layer due to the atomic mixing and rapid resolidification of the target describes in more detailed in the following section.

\subsection{Experimental characterization and atomistic modeling of interfacial void formation and detachment in short pulse laser-processing of metal surfaces covered by transparent overlayers}

\subsubsection{Introduction}

The conditions of spatial confinement realized in short pulse laser interactions with an absorbing substrate covered by an optically transparent solid overlayer have a number of important implications on the materials response to the laser energy deposition, ranging from the enhancement of laser-induced pressure to the suppression of the ablation plume expansion and melt expulsion. The unique characteristics of the spatially confined laser interactions have attracted attention of the research community and have been demonstrated to be beneficial for a number of practical applications, such as laser shock peening [215, 216, 218], laser-induced forward transfer [219-221], thin film patterning/scribing [222, 223], and the generation of highquality surface structures [57, 239].

The physical understanding of the effect of the spatial confinement on laser-induced processes is largely based on the results of theoretical analysis of the evolution of pressure generated under conditions of spatially-confined laser ablation [216, 223] and continuum-level modeling of thermo-elastic deformation of metal films irradiated through transparent substrates [218, 226, 227]. These studies have recently been complemented by atomistic molecular dynamics (MD) simulations of short pulse laser interactions with bulk metal substrate covered by a transparent overlayer [56]. The atomistic simulations have provided important insights into the effect of the spatial confinement on the kinetics and mechanisms of laser-induced phase transformations. In particular, the spatial confinement is found to suppress the generation of unloading tensile wave, decrease the maximum depth of melting, prevent cavitation or spallation in the transiently melted region of the metal substrate, and enhance the probability of nucleation and growth of new crystallites at the metal-overlayer interface. At higher laser fluences, when the surface region of the metal substrate is brought into the supercritical state, the confinement 
by the overlayer prevents the expansion and phase decomposition of the supercritical fluid, which undergoes rapid cooling to the liquid state, contraction, and detachment from the overlayer. The relatively small lateral size of the computational systems used in the first simulations [56], however, prevented detailed analysis of some of the important processes inferred from the simulation results, such as the evolution of voids leading to the detachment at the metal-overlayer interface or the nucleation and growth of multiple crystallites leading to the formation of nanocrystalline structure in the interfacial region.

In the study reported in the section, we extend the initial computational analysis that is discussed in section 5.1 and presented in Ref. [56] to a larger system and perform MD simulations targeted at revealing the microscopic mechanisms responsible for the formation of interfacial voids and/or partial detachment of the overlayer from the metal substrate. The laserinduced processes in the presence of pre-existing interfacial voids generated by previous laser pulses or produced in the course of the deposition of the overlayer are also investigated in an MD simulation. The computational analysis of the generation of interfacial voids is supplemented by a systematic experimental characterization of laser-induced modification of $\mathrm{Al}$ substrates covered by silica overlayers. The results of the experimental investigation are discussed first, in Section 5.2.2, and are followed by presentation of computational predictions in Section 5.2.3 and a brief summary of the main conclusions of the combined experimental and computational study in Section 5.2.4.

\subsubsection{Experimental characterization of laser modification of metal- overlayer targets}

The experimental study is performed for an Al substrate covered by a silica overlayer. The sample used in the experiments has lateral dimensions of $5.1 \times 5.1 \mathrm{~cm}^{2}$ and a thickness of 1 $\mathrm{mm}$. It is prepared by cold rolling of an $\mathrm{Al}$ (3003-H14) plate resulting in a surface roughness of approximately $1 \mu \mathrm{m}$, followed by chemical vapor deposition of a $10 \mu \mathrm{m}$ thick silica coating. Single pulse irradiation of different areas on the sample is performed by IR $1064 \mathrm{~nm}$ laser system at five incident laser fluences of $0.97,1.15,1.28,3.84$, and $7.69 \mathrm{~J} / \mathrm{cm}^{2}$, with a laser pulse duration of $10 \mathrm{ps}$, and a Gaussian $1 / \mathrm{e}^{2}$ laser spot diameter of $50 \mu \mathrm{m}$.

The nature and the extent of the laser-induced structural modification/damage of the metal substrate and the overlayer are characterized by optical and scanning electron 
microscopies. The images obtained by focusing the optical microscope on the outer surface of the silica overlayer and the metal-overlayer interface are shown in Figs. 5.10(a) and 5.10(b), respectively. Based on the visual analysis of these images, one can define the laser affected zone as the area that exhibits a clear optical distinction as compared to the surrounding regions. As expected, the size of the laser affected zones increases with increasing laser fluence. No surface damage to the silica overlayer is observed at the three lower laser fluences $(0.97,1.15$, and 1.28 $\mathrm{J} / \mathrm{cm}^{2}$ ), whereas a partial removal and a complete ablation of the transparent overlayer can be seen at the two highest laser fluences of 3.84 and $7.69 \mathrm{~J} / \mathrm{cm}^{2}$, respectively.

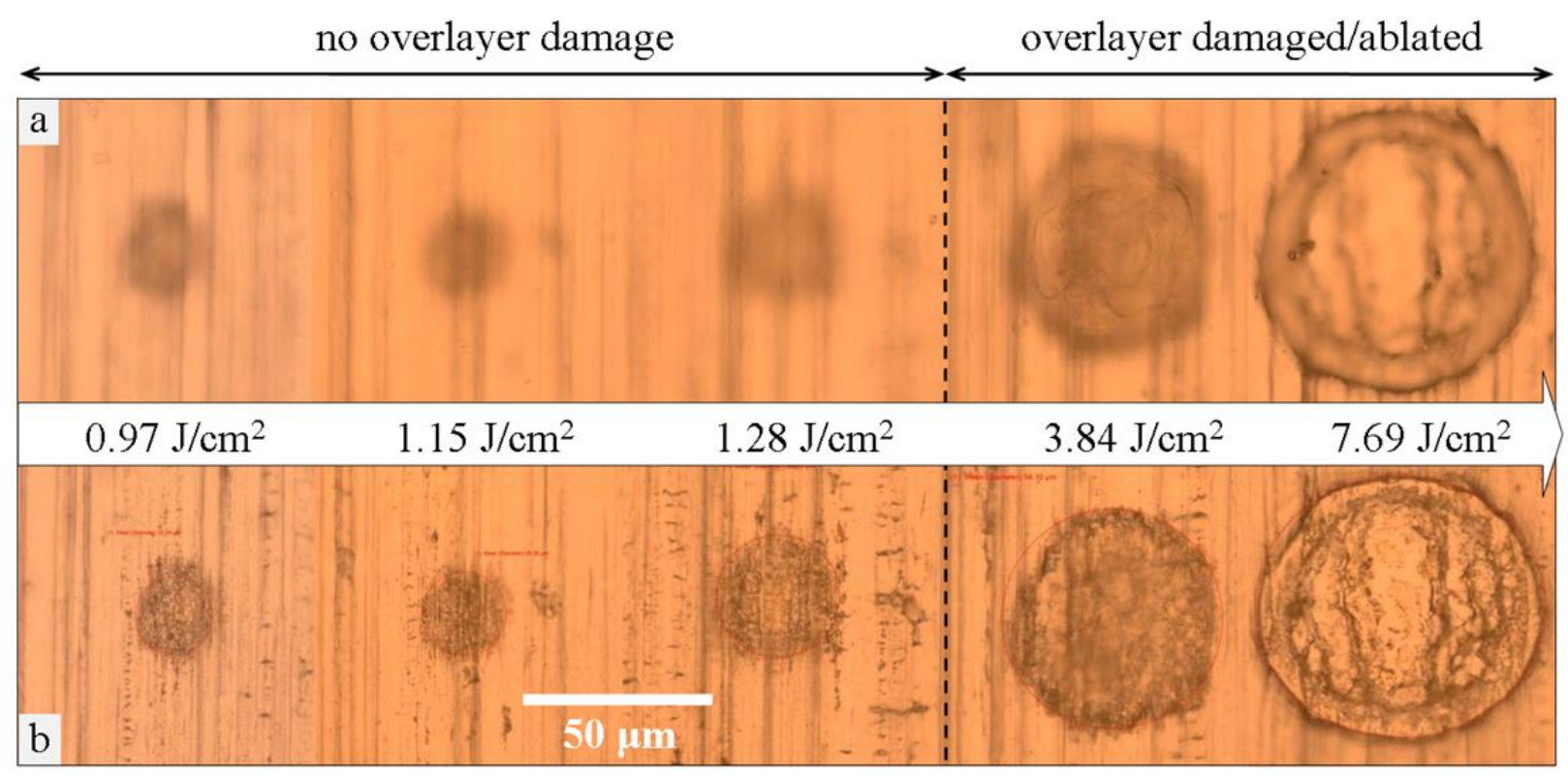

Fig. 5.10. Optical microscope images obtained by focusing microscope lenses on the surface of silica overlayer (a) and the Al-overlayer interface (b) of Al-silica samples irradiated by $10 \mathrm{ps}$ laser pulses at five incident laser fluences indicated in the figure. The black dashed line separates the irradiation conditions that do not result in any visible damage to the overlayer from the ones when cracking/chipping or complete removal of the overlayer is observed. The nature of the target modification is further illustrated by SEM images shown in Fig. 5.11.

The scanning electron microscopy (SEM) images reveal more detailed information on the nature of the laser-induced structural modification or damage to the targets. The SEM images of the vertical cross sections of the irradiated spots prepared by focused-ion-beam (FIB) milling are shown in Fig. 5.11(a-e) for incident fluences of 0.97 and $3.84 \mathrm{~J} / \mathrm{cm}^{2}$. 


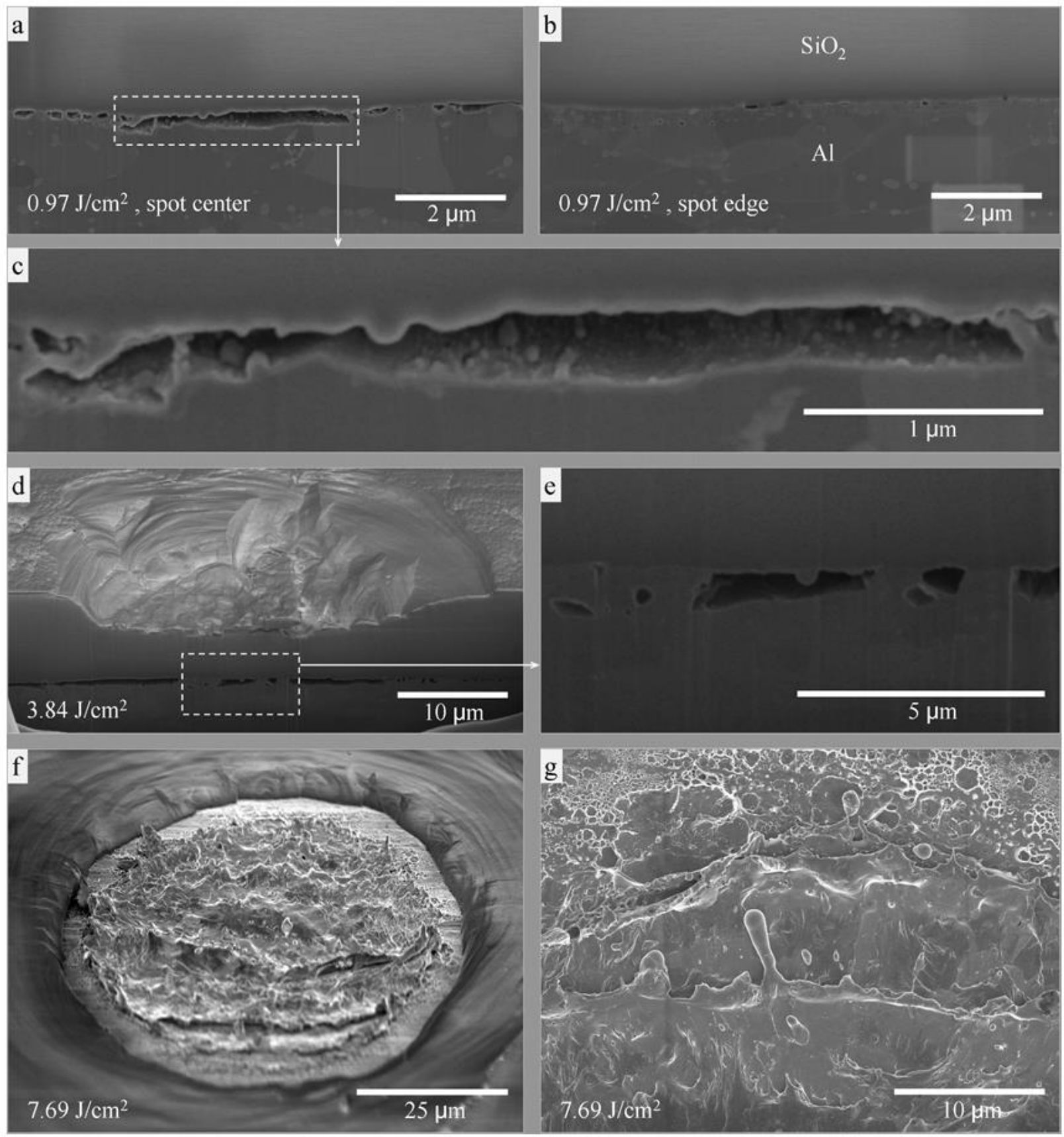

Fig. 5.11. SEM images of Al-silica samples irradiated by 10 ps laser pulse at three incident laser fluences of $0.97 \mathrm{~J} / \mathrm{cm}^{2}(\mathrm{a}-\mathrm{c}), 3.84 \mathrm{~J} / \mathrm{cm}^{2}(\mathrm{~d}, \mathrm{e})$, and $7.69 \mathrm{~J} / \mathrm{cm}^{2}(\mathrm{f}, \mathrm{g})$. The images in (a-e) show vertical cross sections of the irradiated spots prepared by FIB milling that reveal the interfacial voids and partial detachment at the metal-overlayer interface. The images in $(f, g)$ show the metal surface exposed by complete removal of the overlayer at a fluence of $7.69 \mathrm{~J} / \mathrm{cm}^{2}$. 
At the lowest fluence of $0.97 \mathrm{~J} / \mathrm{cm}^{2}$, the laser-induced changes are limited to the generation of elongated micron-scale voids at the Al-silica interface. The voids observed in the central part of the spot (Fig. 5.11(a)) are larger than the ones at the spot edge (Fig. 5.11(b)) and result in a partial detachment of the overlayer from the metal substrate. Interestingly, some of the small voids appear inside the $\mathrm{Al}$ substrate, just beneath the Al-overlayer interface. The SEM images also reveal the presence of nanoscale roughness or nanoparticles on the surface of large voids, as can be seen in the enlarged view of the largest void shown in Fig. 5.11(c). The mechanisms of the void formation and the origin of the nanoscale roughness are investigated in the simulations discussed in Section 5.2.3.

As the laser fluence increases, the size of the voids and the area affected by the detachment also increase, as can be seen from the SEM images shown in Fig. 5.11(d,e) for a laser fluence of $3.84 \mathrm{~J} / \mathrm{cm}^{2}$. Moreover, the irradiation at this fluence also damages the silica overlayer and creates an approximately $5.5 \mu \mathrm{m}$ deep crater at the outer surface of the overlayer. Since the laser fluence of $3.84 \mathrm{~J} / \mathrm{cm}^{2}$ is substantially lower than the damage threshold in bulk fused silica [240], the partial removal of the overlayer cannot be attributed to the direct optical damage in the fused silica. A plausible explanation of the damage to the overlayer may be proposed based on the results of the simulations discussed below, in Section 5.2.3, which suggest that a high, on the order of tens of GPa, compressive pressure is generated near the metal surface by the spatially confined laser excitation. A compressive wave driven by the relaxation of laserinduced pressure propagates from the interfacial region towards the surface of the overlayer, transforms into tensile wave upon reflection from the free surface of the overlayer and may cause spallation when the magnitude of the tensile wave exceeds the dynamic strength of the silica glass [241].

At the highest laser fluence of $7.69 \mathrm{~J} / \mathrm{cm}^{2}$, the pressure created by the laser excitation of the metal substrate is sufficiently high to completely remove the $10 \mu \mathrm{m}$ thick silica overlayer, as can be seen from Fig. 5.11(f,g). The removal of the overlayer makes it possible for the hot metal region to expand and undergo rapid decomposition into vapor and liquid droplets, similar to ablation in a background gas environment or in vacuum. The explosive decomposition and ejection of a top part of the metal substrate create conditions for rapid cooling and solidification of the remaining target. The rapid solidification, in turn, freezes transient liquid structures generated in the ablation process [24] and results in the formation of complex surface 
morphology featuring nanospikes and ripples that are similar to the ones commonly produced by laser processing in vacuum, e.g., [33, 242, 243].

\subsubsection{Atomistic simulation of interfacial void formation}

The dynamic processes responsible for the generation of the interfacial voids and partial detachment of the overlayer from the metal substrate are investigated in a large-scale MD simulation performed for a Ag substrate covered by a thick silica glass overlayer. The choice of $\mathrm{Ag}$ rather than $\mathrm{Al}$ used in the experiments described in Section 5.2.2 is defined by the availability of the results of smaller-scale atomistic simulations performed for the Ag-silica system, which have already identified the irradiation regime where the overlayer detachment can be expected to occur [56]. We note, however, that the phenomena of the generation of interfacial voids and detachment of a transparent overlayer from an absorbing substrate appear to be general and have been observed for various systems, such as a $\mathrm{Si}$ substrate covered by a thermally growth $\mathrm{SiO}_{2}$ layers [244] or Ni and Si substrates covered by a polymer confinement layer [57]. Therefore, we expect the computational insights into the mechanisms of the interfacial void formation to be applicable to various material systems where a confinement layer is used to control the laser processing conditions.

\subsubsection{Computational model}

The atomistic simulations of laser interactions with a Ag substrate covered by a transparent overlayer are performed with a hybrid computational model [118] combining the classical MD method with a continuum-level description of the laser excitation and subsequent relaxation of the conduction-band electrons based on so-called two temperature model (TTM) [90]. A part of the transparent overlayer adjacent to the metal substrate is also simulated with a simplified MD model fitted to provide an adequate description of the experimental values of elastic constants, melting temperature, and density of fused silica, as well as the work of adhesion at the Ag-silica interface. The dynamic pressure-transmitting boundary conditions [56, $110,130]$ are applied at the bottom of the TTM-MD region of the metal substrate and at the top of the MD part of the overlayer to simulate non-reflective propagation of the laser-induced pressure waves from the interfacial region to the bulk of the metal substrate and towards the surface of the overlayer, respectively. The reflection of the pressure wave from the top surface of 
the overlayer is not considered in the simulations since the time required for the propagation of the wave through a $10 \mu \mathrm{m}$ thick silica overlayer and back to the interfacial region is longer than the time-scale of the processes considered in the simulations. A complete description of the computational model is provided in section 5.1 and Ref. [56] and below we only provide the parameters of the computational setup used in the simulations reported in this section.

Taking advantage of the capabilities of the computationally-efficient parallel implementation of the computational model, one of the simulations reported below, in Section 5.2.3.2, is performed for a large computational system composed of more than 330 million atoms. The lateral (parallel to the Ag-silica interface) dimensions of the system are $100 \mathrm{~nm} \times 100$ $\mathrm{nm}$ and periodic boundary conditions are applied in these directions. In the direction normal to the interface, the TTM-MD and MD representations of the Ag substrate and the silica overlayer extend to $500 \mathrm{~nm}$ for $\mathrm{Ag}$ ( $\sim 303$ million atoms) and $200 \mathrm{~nm}$ for silica ( 27 million atoms) from the interface. In another simulation, targeted at evaluation of the effect of pre-existing interfacial voids on the laser induced processes and discussed in Section 5.2.3.3, a much smaller lateral size of the system, $4.1 \mathrm{~nm} \times 4.1 \mathrm{~nm}$, a thickness of the TTM-MD part of the Ag substrate of $411 \mathrm{~nm}$, and a thickness of the MD part of the silica overlayer of $137 \mathrm{~nm}$ are used.

In both simulations, the continuum part of the TTM-MD model, in which the electron heat conduction is described by the conventional TTM, is extended to the depth of $2.5 \mu \mathrm{m}$ from the interface to ensure that no substantial temperature changes are observed at the bottom of the continuum region by the end of the simulation. The choice of the parameters used in the TTM equation for the electron temperature of $\mathrm{Ag}$ is explained in Ref. [141] and the interatomic interactions in the Ag part of the model are described by the embedded atom method (EAM) potential in the form proposed in Ref. [128], with a cut-off function [209] that smoothly brings the interaction energies and forces to zero at a cut-off distance of $5.5 \AA$ added to the potential. Before applying the laser irradiation, the computational systems are equilibrated at $300 \mathrm{~K}$ for 200 ps.

\subsubsection{Generation of the interfacial voids and overlayer detachment}

The physical processes responsible for the appearance, growth, and percolation of the interfacial voids leading to the detachment of the overlayer from the metal substrate are discussed in this section based on the results of a large-scale simulation performed for irradiation 
conditions identified in smaller-scale simulations [56] as conditions leading to the overlayer detachment. The metal-overlayer target is irradiated by a 10 ps laser pulse at an absorbed laser fluence of $0.4 \mathrm{~J} / \mathrm{cm}^{2}$, which is about twice higher than the threshold fluence for the onset of phase explosion in the case of laser ablation of a bare Ag target in vacuum. While the uncertainty with respect to the reflectance of the metal-overlayer interfaces in experiments discussed in Section 5.2.2 does not allow us to reliably convert the values of the incident laser fluence to the absorbed fluence, the conditions of the simulation performed at the absorbed fluence that is about $40-50 \%$ above the threshold for the generation of the interfacial voids [56] can be expected to be comparable to the ones in the lower fluence experiments (incident fluences of $0.97-1.28$ $\mathrm{J} / \mathrm{cm}^{2}$ ) where partial detachment at the interface (Fig. 5.11(a-c)) but no damage to the overlayer (Fig. 5.10) are observed.

The processes responsible for the structural modification of the metal-overlayer target are largely defined by the rapid evolution of temperature and pressure in the vicinity of the region of the laser energy deposition, shown in the form of contour plots in Fig. 5.12. Following the laser excitation of the conduction band electrons within the optical absorption depth of the Ag substrate, the energy of the excited electrons is rapidly redistributed through the ballistic and diffusive electron energy transport and transferred to the lattice vibrations due to the electronphonon coupling. This energy transfer leads to a rapid temperature increase within $\sim 200 \mathrm{~nm}$ deep region of the metal substrate, Fig. 5.12(a). The temperature quickly exceeds the limit of crystal stability against the onset of massive homogeneous meting [162] and triggers a rapid (within several picoseconds) collapse of the highly superheated crystal lattice. Additional slower melting down to the depth of $\sim 340 \mathrm{~nm}$ below the Ag-overlayer interface then proceeds through the propagation of the melting front [143] from the homogeneously melted part of the substrate. Following the rapid melting, the temperature of liquid Ag adjacent to the interface continues to increase due to the energy transfer from the hot electrons and reaches the maximum of $\sim 8200 \mathrm{~K}$ by $40 \mathrm{ps}$ (15 ps after the time of the peak intensity of the Gaussian laser pulse), thus bringing the top part of the Ag substrate to the supercritical state. 

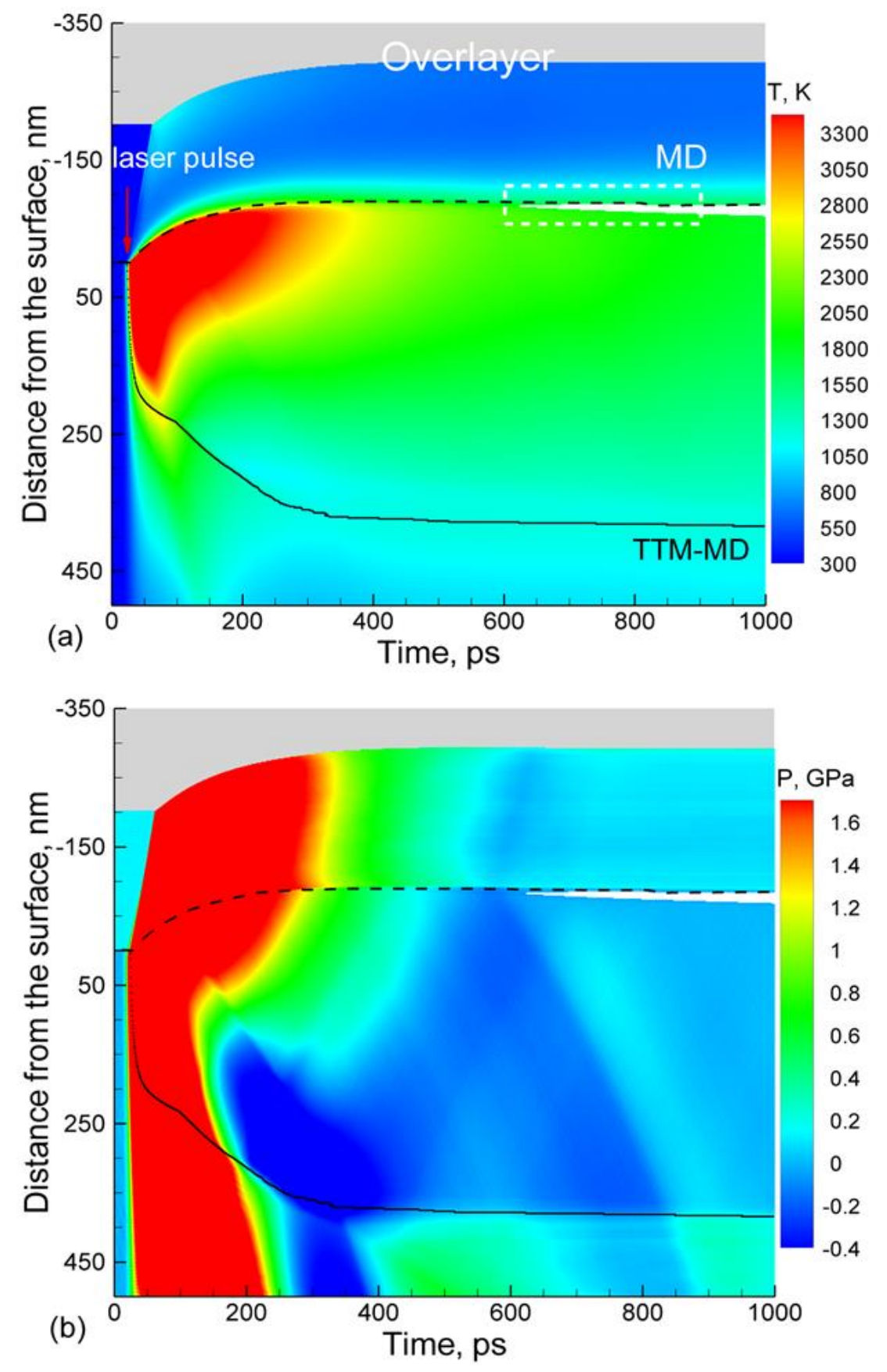

Fig. 5.12. Contour plots showing the spatial and temporal evolution of (a) temperature and (b) pressure predicted in a simulation of a Ag-overlayer target irradiated by a $10 \mathrm{ps}$ laser pulse at an absorbed fluence of $0.4 \mathrm{~J} / \mathrm{cm}^{2}$. To ensure complete deposition of the laser energy, the peak intensity of the Gaussian laser pulse is shifted to $25 \mathrm{ps}$ from the beginning of the simulation, as shown by the red arrow in (a). The black solid curve line separates the melted region from the crystalline part of the Ag target. The black dashed line tracks the location of the Ag-overlayer interface. The area where the density of the material drops below $10 \%$ of the initial density of the solid Ag target is blanked in the plots. The dashed white rectangle in (a) marks the time range and location of the slice of the computational system for which snapshots of atomic configurations are shown in Fig. 5.13. 
The rapid temperature increase during the first $40 \mathrm{ps}$ of the simulation takes place under conditions of stress confinement $[113,120,149,152]$ and results in a build-up of strong compressive-stresses, as can be seen from Fig. 5.12(b). The compressive pressure reaches the maximum level of $\sim 30 \mathrm{GPa}$ at the depth of $\sim 60 \mathrm{~nm}$ below the interface by the time of $35 \mathrm{ps}$ and then relaxes by expanding the hot region of the metal substrate and driving two compressive pressure waves, one deeper into the metal substrate and another into the silica overlayer. In contrast to the laser ablation of a bare metal surface, where the expansion of the surface region proceeds in the form of an explosive decomposition of the superheated liquid into a mixture of vapor and liquid droplets [24, 113, 120, 134], the phase decomposition is suppressed by the presence of the overlayer and the supercritical fluid near the interface is gradually cooled to the liquid state mostly by the electronic heat transfer to the bulk of the metal substrate.

Since the acoustic impedance of the overlayer material is more than twice smaller than the one of $\mathrm{Ag}$, the expansion of the metal substrate against the softer overlayer also produces a weak unloading wave propagating from the interface to the bulk of the $\mathrm{Ag}$ substrate. Partial reflection of the unloading wave from the melting front generates a transient spike of the tensile stresses up to about $-1.1 \mathrm{GPa}$ near the solid-liquid interface, at a depth of $\sim 330 \mathrm{~nm}$. The relatively low temperature of liquid near the melting front (close to the equilibrium melting temperature of the EAM Ag at zero pressure, $T_{m}=1139 \mathrm{~K}$ [141]) and a short time of the spike of the tensile stresses preclude cavitation from taking place in the region of the maximum tensile stresses. When the reflected part of the tensile wave reaches the metal-overlayer interface at $\sim 600 \mathrm{ps}$, however, the much lower tensile stresses of about $-0.12 \mathrm{GPa}$ do induce nucleation of voids in the interfacial region weakened by melting of $\sim 10 \mathrm{~nm}$ part of the overlayer and relatively high temperature of the interfacial region, $\sim 1.7 T_{m}$ at the time of the arrival of the tensile wave. The growth of the voids results in the appearance of a region of reduced density at the metal-overlayer interface that shows up as a white gap in the contour plots in Fig. 5.12. The growth of the voids is facilitated by continuous cooling and contraction of the top region of the metal substrate that contribute to the expansion of the cavitation zone, elongation of the liquid bridges connecting the metal substrate to the overlayer and eventual detachment of the overlayer. 
A visual picture of the nucleation and growth of the voids at the Ag-overlayer interface is provided by a series of snapshots of atomic configurations shown in Fig. 5.13. The snapshots are shown for a $74 \mathrm{~nm}$ thick slice of the computational cell adjacent to the interface, as outlined in Fig. 5.12(a). The first snapshot is shown for a time of $600 \mathrm{ps}$, when a $10 \mathrm{~nm}$ deep part of the silica overlayer is melted and the first small voids appear at the Ag-overlayer interface in response to the raise of the tensile stresses (Fig. 5.12(b)). Gradually, growth and coalescence of the voids lead to the formation of liquid bridges connecting the overlayer and metal substrate, with some of the bridges breaking as the gap between the overlayer and the metal substrate grows. By the end of the simulation at $1 \mathrm{~ns}$, three liquid bridges still connect the overlayer with the Ag substrate. Note that although vapor-phase atoms can be clearly seen to fill up the cavitation region, the vapor pressure of $1.7 \mathrm{MPa}$, estimated from the temperature and density of the vapor in the ideal gas approximation, is negligible as compared to the variation of stresses shown in Fig. 5.12(b). The generation and growth of the interfacial voids, therefore, can be squarely attributed to the dynamic relaxation of the laser-induced stresses proceeding simultaneously with rapid expansion and contraction of the superheated surface region of the metal substrate, with the release of the vapor playing an insignificant role.

The evolution of voids in the interfacial region is further illustrated in Fig. 5.14, where top-view projections of all the voids are shown. The voids are identified by dividing the computational cell into small cubic boxes with volumes of $3.4 \mathrm{~nm}^{3}$ and identifying voids as regions composed of boxes with density lower than $10 \%$ of the initial density of Ag. The voids are then plotted in Fig. 5.14 as dark regions, whereas the white region represents the liquid bridges connecting the Ag substrate with the overlayer. As discussed above, the nucleation of small voids at $\sim 600$ ps coincides with the arrival of the tensile wave to the Ag-overlayer interface and the contraction of the top part of the metal substrate due to the cooling process. Following the nucleation, the voids grow and coalesce, thus reducing the number and the total crosssectional area of the connecting liquid bridges. 

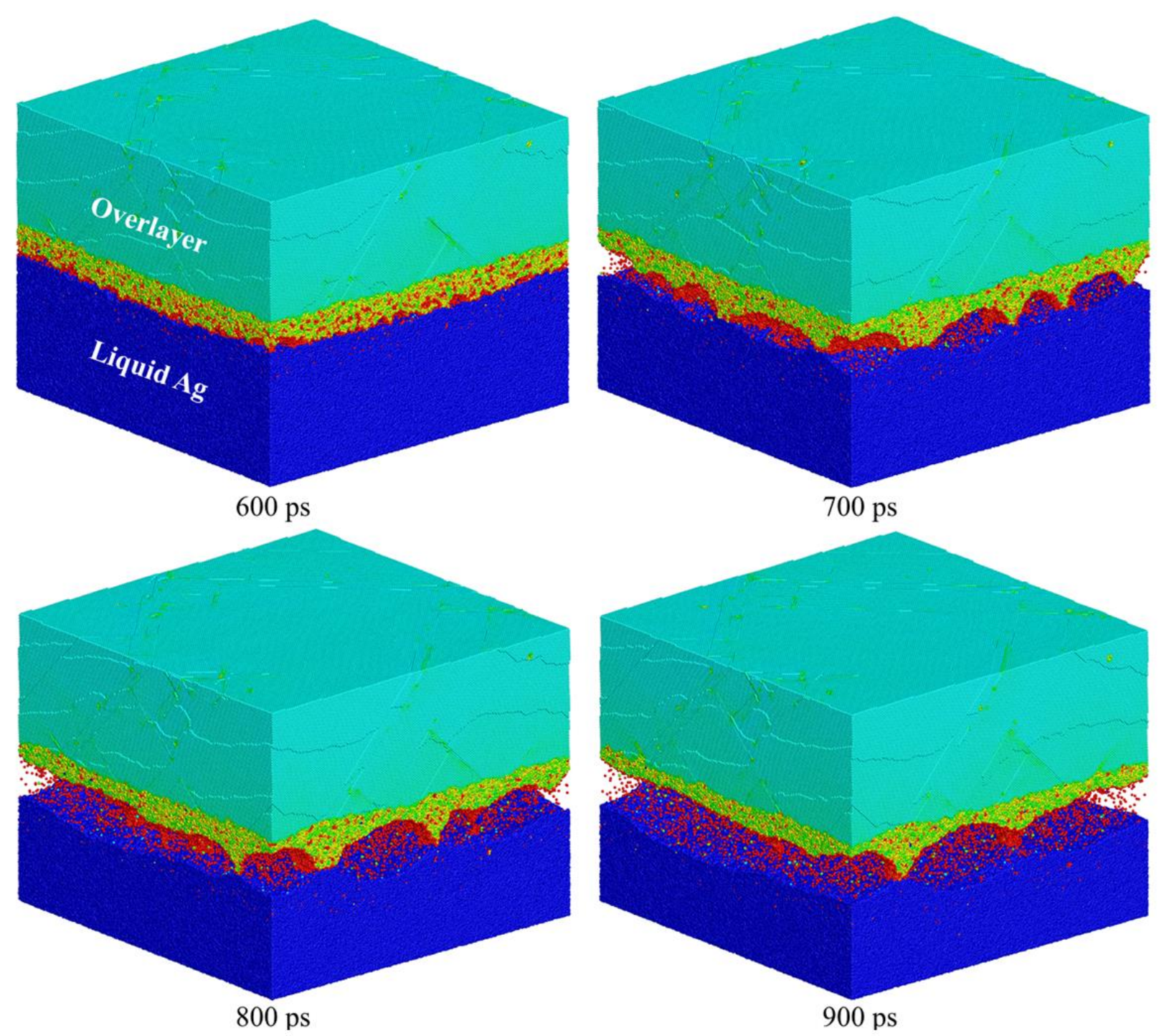

Fig. 5.13. Snapshots of atomic configurations obtained in a simulation of a Ag-overlayer target irradiated by a 10 ps laser pulse at an absorbed fluence of $0.4 \mathrm{~J} / \mathrm{cm}^{2}$. Only a $74 \mathrm{~nm}$ thick slices of the computational system adjacent to the Ag-overlayer interface are shown in the snapshots. The atoms are colored according to their potential energies, so that the dark blue color represents liquid Ag, light green and yellow colors represent the solid and melted parts of the overlayer, and the vapor phase atoms are red. 
The formation of the interfacial voids and partial detachment of the overlayer from the metal substrate are consistent with experimental observations presented in Section 5.2.2. The formation of the liquid bridges connecting the metal substrate with the overlayer may be responsible for generation of the nanoscale roughness on the surface of large voids observed in experiments, Fig. 5.11(c).

While the relatively weak adhesion between the silica overlayer and Ag leads to the early breakup of the liquid bridges in the simulation (the three remaining bridges observed in the simulation at $1 \mathrm{~ns}$ are likely to break up upon further expansion of the gap between the metal substrate and overlayer), a stronger metal-silica interaction, faster cooling due to the threedimensional heat transfer under experimental conditions, and the restraining effect of the colder periphery of the laser spot, where the conditions for the detachment are not achieved, on the outward motion of the central part of the overlayer may stabilize the bridges and facilitate nucleation of some of the voids below the metal-overlayer interface. The solidification process may then capture the voids/bridges, solidify the transient surface roughness generated by the evolving liquid bridges, and produce the interfacial structures shown in the experimental images in Fig. 5.11. An additional scenario for the generation of voids inside the metal substrate, below the metal-overlayer interface, can be suggested based on the results of a simulation of laser interaction with a system containing a pre-existing interfacial void that is described below. 


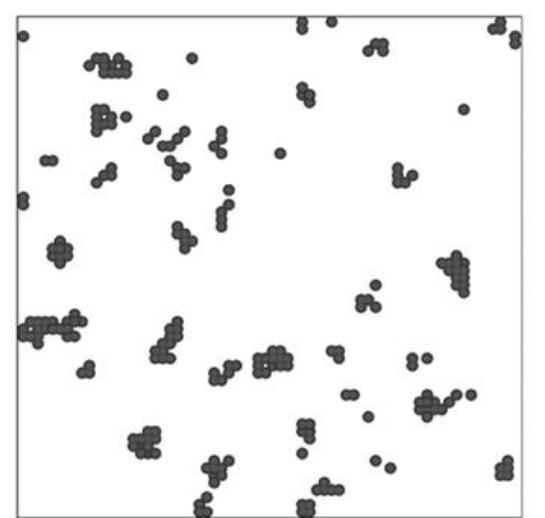

$$
600 \mathrm{ps}
$$

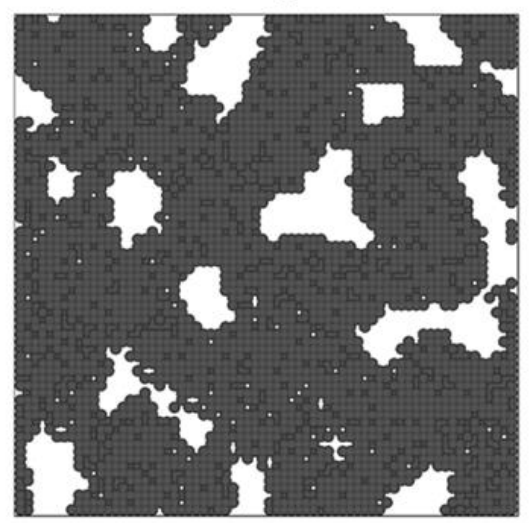

$750 \mathrm{ps}$

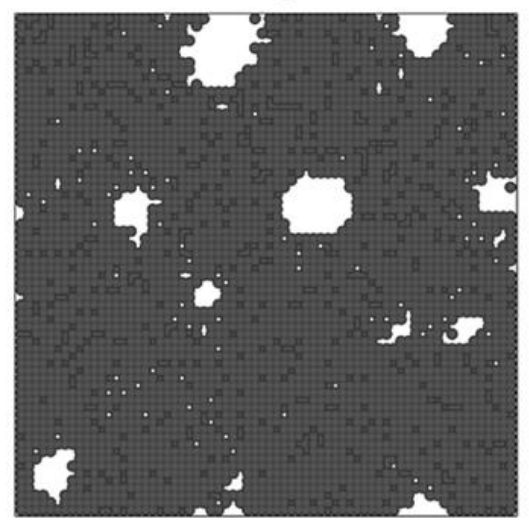

$900 \mathrm{ps}$

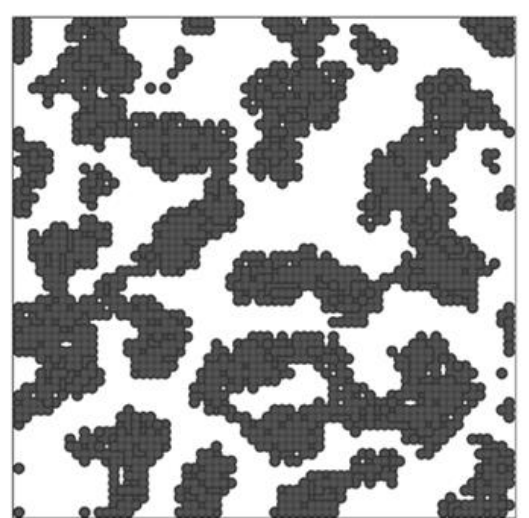

$650 \mathrm{ps}$

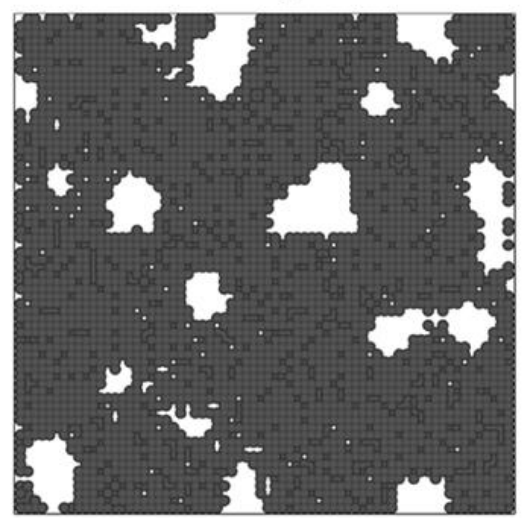

$800 \mathrm{ps}$

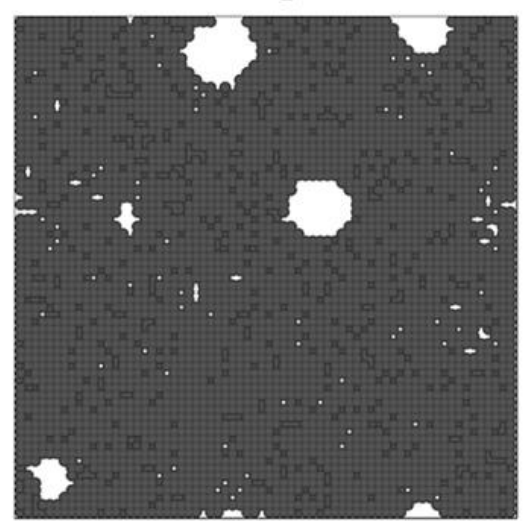

$950 \mathrm{ps}$

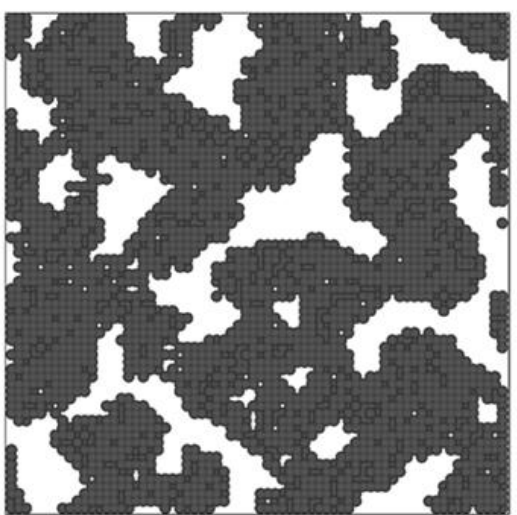

$700 \mathrm{ps}$

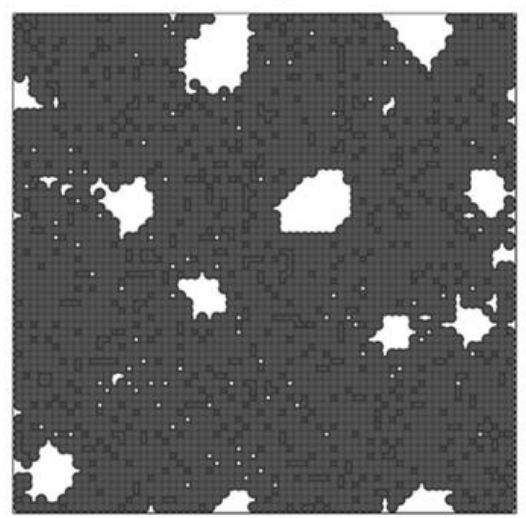

850 ps

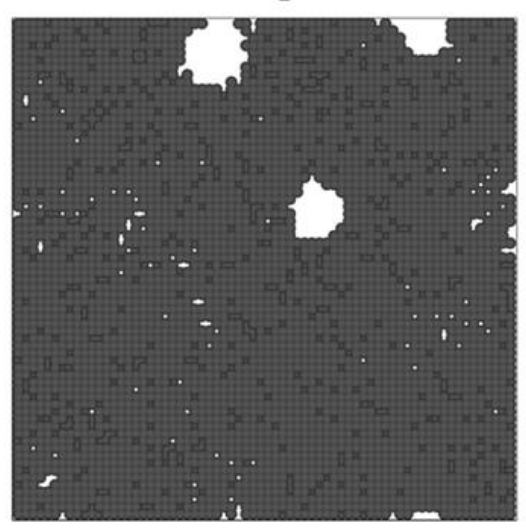

$1000 \mathrm{ps}$

Fig. 5.14. Top-view projections of the voids evolving in the Ag-overlayer interfacial region in the simulation illustrated by Figs. 5.12 and 5.13. The dark regions show the areas where the material density drops below $10 \%$ of the initial density of solid Ag, whereas the white regions represent the connections between the metal substrate and the overlayer. 


\subsubsection{The effect of pre-existing interfacial voids}

In contrast to the perfect metal-overlayer interface considered in the previous section, real experimental samples may contain small interfacial gaps or voids formed in the process of sample preparation. Moreover, the voids generated by the first laser pulse may affect the response of the target to irradiation by subsequent laser pulses in the multi-pulse irradiation regime. To investigate the effect of pre-existing interfacial voids on the laser-induced processes, a simulation of a system with a $50 \mathrm{~nm}$ gap between the transparent overlayer and $\mathrm{Ag}$ substrate has been performed for a smaller computational system (lateral size of $4.1 \mathrm{~nm} \times 4.1 \mathrm{~nm}$ ) and the same irradiation condition as in the simulation discussed above, in Section 5.2.3.2.

The evolution of temperature, pressure and density in the irradiated system is presented in Fig. 5.15. The presence of the void makes it possible for the hot metal region to expand and generate an unloading wave propagating from the interfacial region and initiating the material decomposition down to the depth $\sim 180 \mathrm{~nm}$. As a result, the initial stage of the material response to the laser excitation is similar to the one of a target with a free surface, when the top surface region undergoes an explosive decomposition into a mixture of vapor and small droplets while the generation of voids in a deeper and colder melted region is driven by the tensile stresses associated with unloading wave $[24,60,113,120,134]$. In the present simulation, however, the collision of the ejected material with the overlayer interrupts the expansion of the ablation plume, converts the kinetic energy of directed motion of the plume into thermal energy, and creates a highly energetic supercritical fluid with the maximum temperature and pressure values reaching $10,000 \mathrm{~K}$ and $8 \mathrm{GPa}$ by the time of $70 \mathrm{ps}$.

The pressure exerted by the hot supercritical metal pushes the overlayer up by $\sim 100 \mathrm{~nm}$ and launches a compaction wave that stops the material decomposition initiated by the unloading wave and leads to the collapse of all but the deepest subsurface voids generated in the course of the initial material expansion. Each of the collapsing voids generates a pressure pulse consisting of compressive and unloading/tensile components that can be seen in the contour plots in Fig. 5.15 . 

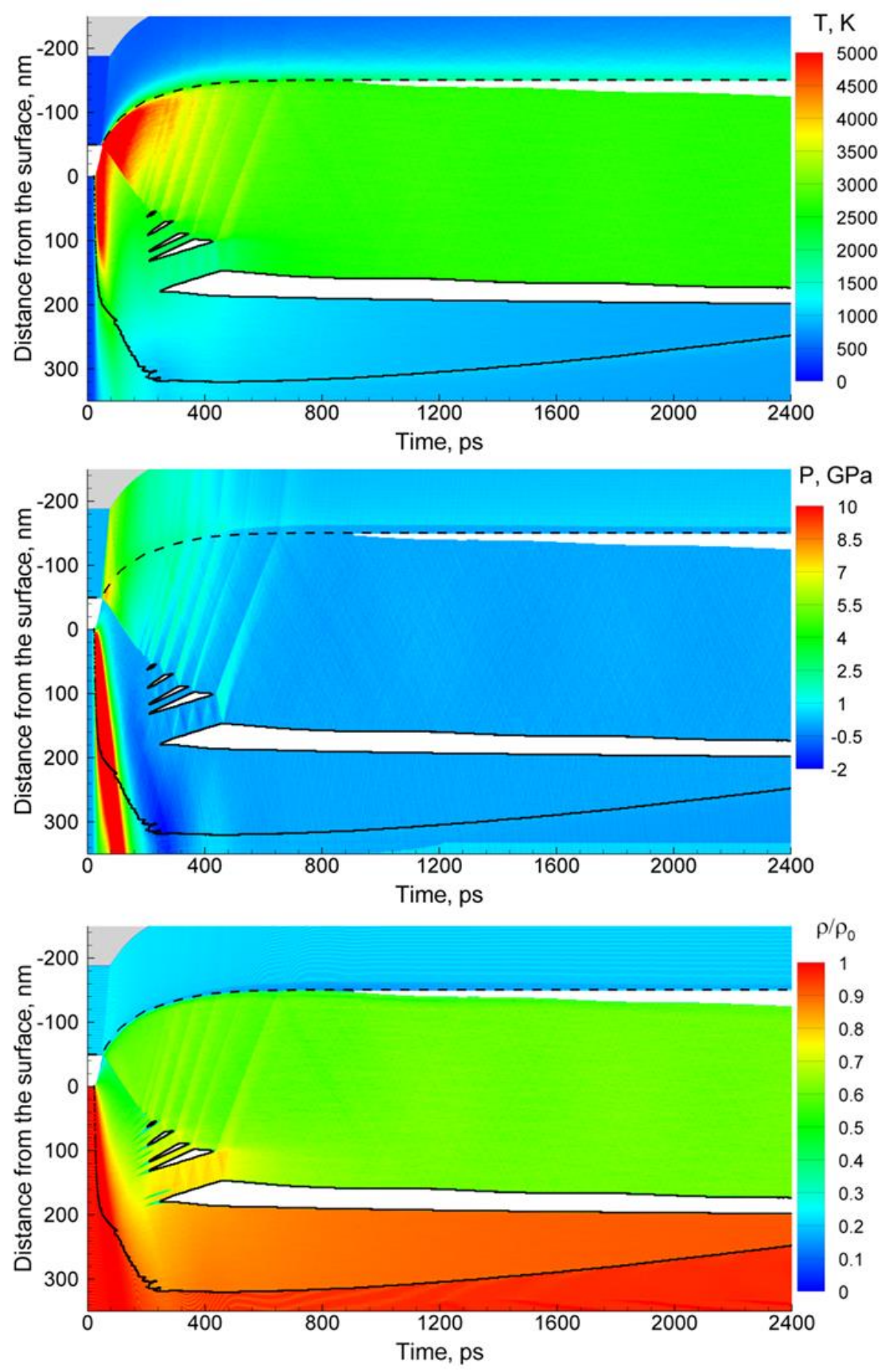

Fig. 5.15. Temperature, pressure and density contour plots obtained in a simulation of bulk Ag substrate covered by a transparent overlayer with an initial $50 \mathrm{~nm}$ thick gap between the overlayer and the $\mathrm{Ag}$ substrate. The Ag-overlayer target is irradiated by a $10 \mathrm{ps}$ laser pulse at an absorbed laser fluence of 0.4 $\mathrm{J} / \mathrm{cm}^{2}$. Areas where the density of the material drops below $10 \%$ of the initial density of the solid $\mathrm{Ag}$ target are blanked in the plots. The black curves outline voids inside the material and separate the melted region from the crystalline part of the $\mathrm{Ag}$ target. The black dashed line shows the position of the $\mathrm{Ag}$ overlayer interface. 
The electronic heat transfer to deeper regions of the metal substrate combined with the fluid expansion and the energy loss on the mechanical work done by displacing the overlayer (the latter can be estimated to be on the order of $400 \mathrm{~J} / \mathrm{m}^{2}$, which roughly corresponds to the thermal energy change upon cooling of a $50 \mathrm{~nm} \mathrm{Ag}$ layer by $\sim 2500 \mathrm{~K}$ ) leads to a rapid cooling of the interfacial part of the metal substrate from the supercritical state with temperature of $\sim 10^{4} \mathrm{~K}$ down to the regular liquid with a temperature of $2700 \mathrm{~K}$ by the time of $900 \mathrm{ps}$.

At $900 \mathrm{ps}$, the combined effect of the contraction of the rapidly cooled liquid Ag and an arrival of a weak tensile stress wave generated at the onset of the collapse of the last void (the maximum tensile stresses at the interface reach the level of about $-50 \mathrm{MPa}$ at $850 \mathrm{ps}$ ) results in the detachment at the metal-overlayer interface of the metal substrate from the overlayer. Given the slower $(\sim 11 \mathrm{~m} / \mathrm{s})$ downward motion of the detached liquid layer as compared to the faster $(\sim 56 \mathrm{~m} / \mathrm{s})$ advancement of the solidification front, one can expect the solidification front to reach the location of the void $\sim 1.5 \mathrm{~ns}$ earlier. While the small lateral size of the computational system used in this simulation does not allow us to simulate the void capture and stabilization by the solidification front, such process has been observed in larger-scale simulations of laser interaction with an Ag target in vacuum [245]. Thus, the results of the simulation discussed above indicate that the presence of pre-existing interfacial voids may facilitate generation of voids at some depth below the interface, as observed in experimental images, Fig. 5.11(a-e).

\subsubsection{Summary}

The characteristic features of short pulse laser processing of metal surfaces under conditions of spatial confinement by a transparent overlayer are investigated in experiments and atomistic simulations, with a particular focus on establishing the mechanisms responsible for the formation of interfacial voids and/or partial detachment of the overlayer from the metal substrate. The dependence of the nature of the laser-induced structural modification and/or damage of the metal-overlayer target on the incident laser fluence is characterized experimentally for $\mathrm{Al}$ substrates covered by silica overlayers. At laser fluences that are close to the threshold for the interfacial void formation, the SEM images reveal the presence of nanoscale roughness on the surface of elongated interfacial voids, formation of small voids inside the metal substrate, and partial detachment of the overlayer from the substrate in the central region of the irradiated laser spot. The increase in the laser fluence leads to the increase in the size of the voids, expansion of 
the area affected by the detachment and, eventually, to the cracking/chipping or complete removal of the overlayer.

The physical processes responsible for the appearance, growth, and percolation of the interfacial voids leading to the detachment of the overlayer from the metal substrate are investigated in a large-scale atomistic simulation performed for a Ag substrate covered by a thick silica glass overlayer. The results of the simulations demonstrate that the generation and growth of the interfacial voids is driven by the dynamic relaxation of laser-induced stresses proceeding simultaneously with rapid phase transformations and temperature variation in the interfacial region. The initial expansion of the top surface region of the metal substrate brought to the supercritical state by the laser energy deposition turns to the contraction caused by the rapid cooling of the region down to the liquid state. The contraction of the surface region combined with additional tensile stresses produced by the arrival of an unloading wave generated at the metal-overlayer interface and partially reflected from the melting front is leading to the void nucleation and growth in the interfacial region. The growth and coalescence of the interfacial voids result in the formation of liquid bridges connecting the overlayer and metal substrate, whereas solidification of the transient liquid structures produced by the breaking bridges may be responsible for the formation of the nanoscale roughness of the internal surfaces of interfacial voids observed in experiments. The results of an additional simulation performed for a system containing a pre-existing interfacial void reveal a complex dynamics of the initial expansion and subsequent compaction of the surface region of the metal substrate and suggest a possible scenario for the formation of voids inside the metal substrate, below the metal-overlayer interface. 


\section{Summary of the dissertation}




\section{Summary of the dissertation}

An extensive computer simulation study of laser interactions with metals is performed with a hybrid computational model combining the classical molecular dynamics method with a continuum description of the laser excitation and subsequent relaxation of the conduction band electrons. The results of the simulations have substantially expanded our understanding of the complex processes occurring under extreme conditions generated by short pulse laser irradiation.

Several research problem have been addressed in this computational study. In the first part, a series of TTM-MD simulations of bulk bcc Cr targets irradiated by $200 \mathrm{fs}$ laser pulses at a broad range of absorbed laser fluences, from 430 to $3830 \mathrm{~J} / \mathrm{m}^{2}$ is performed. This range of laser fluences covers three distinct regimes of the material response to the laser irradiation, namely, melting and solidification of the surface region of the target, photomechanical spallation of liquid layers, and phase explosion and ejection of a superheated surface region. The transitions between the different regimes are identified according to the total amount of ejected material as well as the composition of the ablation plume.

In order to provide a realistic representation of the laser-induced photomechanical spallation, a large-scale simulation of a femtosecond laser pulse irradiation of (001) Cr target is performed for a system consisting of more than 126 million atoms. The results of the simulation reveal the microscopic mechanisms responsible for the photomechanical spallation of a liquid layer from the bulk of the target. The relaxation of strong compressive stresses generated by the rapid laser energy deposition leads to the emergence of an unloading tensile wave, which exceeds the dynamic strength of the material resulting in the nucleation, growth, coalescence, and percolation of sub-surface voids. The evolution of voids leads to the complete spallation of a molten layer from the target and generation of complex surface morphology after complete resolidification of the target. The characteristic features of the surface morphology predicted in the simulation are found to match those observed in laser processing experiments.

A detailed analysis of the surface regions of $\mathrm{Cr}$ targets irradiated by short laser pulses reveals the formation of high density of point defects (mostly vacancies, and some interstitials) in the regions that experienced transient melting and resolidification. The generation of the large numbers of vacancies has important implications on the physical, chemical, and mechanical 
properties of the surface layers. The variation of the vacancy concentration with depth under the irradiated surface is analyzed for each simulation and related to the normalized surface temperature at the end of the solidification process. The concentration of vacancies is also determined in bulk Ni targets with fcc crystal structure, in order to compare the results with the ones obtained for bcc $\mathrm{Cr}$.

In general, there are two mechanisms which may be responsible for the vacancies formation in laser processing of metals: (1) thermally activated generation of vacanciesinterstitial pairs and (2) vacancy formation at the rapidly advancing solidification front. In order to identify the mechanisms responsible for the vacancies formation in the surface region of the irradiated metal targets, a series of MD simulations of the solidification process in $\mathrm{Cr}$ and $\mathrm{Ni}$ systems is performed under well-controlled temperature and pressure conditions. The distribution of the vacancies is analyzed in each atomic plane and related to the velocity of the solidification front. The vacancies diffusivity as well as the time between the vacancy jumps are determined for each system. The simulations reveal that the dominant mechanism responsible for the vacancies formation in both systems is the generation of vacant lattice sites at the solidification front rapidly advancing under conditions of strong undercooling below the equilibrium melting temperature of the target material.

The effect of homogeneous nucleation of new crystallites in the supercooled liquid on the velocity of solidification front and the sensitivity of the computational predictions to the type of interatomic potential used in the simulations are also investigated. The results of two series of MD simulations performed for $\mathrm{Ni}$ described by the embedded atom method potentials in the forms proposed by Foiles, Baskes, and Daw, "EAM-FBD" and Johnson, "EAM-Johnson" potential, are compared with each other. In both cases, the dependence of the solidification front velocity on the normalized temperature shows a linear dependence trend in the high temperature regime, from the melting temperature down to about $0.7 T_{m}$. This behavior changes at low temperatures. While for the EAM-FBD Ni the solidification velocity decreases with decreasing temperature, the velocity predicted for EAM-Johnson Ni remains constant down to a temperature of about $0.1 T_{m}$. The analysis of the results for EAM-FBD Ni reveals that the nucleation and growth of small crystallites inside the undercooling liquid phase slows down the advancement of the solidification front. 
The computational study is also extended to the investigation of the mechanisms responsible for the generation of linear defects, dislocations, and the dependence of the dislocation emission on the crystallographic orientation of the irradiated targets. Three largescale TTM-MD simulations of Cr target with (001), (110), and (111) surfaces are performed. The targets are irradiated by a $50 \mathrm{fs}$ laser pulse at an absorbed laser fluence of $800 \mathrm{~J} / \mathrm{m}^{2}$, which is above the threshold for laser melting but below the threshold for photomechanical spallation. The detailed analysis of the results obtained in the three simulations reveals the formation of unstable partial dislocations (island of stacking faults) in (001) $\mathrm{Cr}$ target, and a high density of perfect dislocations in (110) and (111) Cr targets. Furthermore, the formation of high densities of point defects in the surface regions of the targets is observed in all three simulations.

The general mechanisms of laser melting, spallation, and ablation of metals in vacuum has been extensively studied experimentally, theoretically, and computationally. On the other hand, the effect of strong spatial confinement by a solid transparent overlayer on the laserinduced processes remains largely unexplored. Accordingly, two series of large-scale atomistic simulations are performed for a Ag target, one of them carried out under a vacuum condition and another for a target covered by a transparent overlayer. The material response to the laser energy deposition is studied for a range of laser fluences that, in the absence of the transparent overlayer, covers the regimes of melting and resolidification, photomechanical spallation, and phase explosion of the overheated surface region. In contrast to the irradiation in vacuum, the spatial confinement by the overlayer facilitates generation of sustained high-temperature and pressure conditions near the metal-overlayer interface, suppresses the generation of unloading tensile wave, decreases the maximum depth of melting, and prevents the spallation and explosive disintegration of the surface region of the metal target. At high laser fluences, when the laser excitation brings the surface region of the metal target to supercritical conditions, the confinement prevents the expansion and phase decomposition characteristic for the vacuum conditions and leads to a gradual cooling of the hot compressed supercritical fluid down to the liquid phase and eventual solidification. The target modification in this case is limited to the generation of crystal defects and the detachment of the overlayer from of the metal target.

The mechanisms responsible for the detachment of the overlayer are investigated in an additional large-scale atomistic simulation. The results of the simulation provide detailed information on the nucleation and growth of the interfacial voids driven by the dynamic 
relaxation of laser-induced stresses and proceeding simultaneously with rapid phase transformations and temperature variation in the interfacial region. The growth and coalescence of the interfacial voids result in the formation of liquid bridges connecting the overlayer and the metal substrate, whereas solidification of the transient liquid structures produced by breakup of the liquid bridges may be responsible for the formation of the nanoscale roughness of the interfacial voids observed in experiments. Computational analysis of the effect of pre-existing interfacial voids reveals a complex dynamic picture of the initial expansion and subsequent compaction of the surface region of the metal substrate and suggests a possible scenario for the formation of voids below the metal-overlayer interface. Computational predictions are related to the results of experimental characterization of Al-silica targets modified by single pulse laser irradiation. 


\section{References}

1. Y. Pauleau, “Materials Surface Processing by Directed Energy Techniques”, Elsevier Ltd., Oxford (2006).

2. D. M. Mattox, "Handbook of Physical Vapor deposition (PVD) Processing” Elsevier Inc., Oxford (2010).

3. M. J. Hampden-Smith, and T. T. Kodas, Chemical Vapor Deposition of Metals: Part 1. An Overview of CVD Processes, Chem. Vap. Deposition 1, 8-23 (1995).

4. Y. Yang, K.-H. Kim, and J. L. Ong, A review on calcium phosphate coatings produced using a sputtering process-an alternative to plasma spraying, Biomaterials 26, 327-337 (2005).

5. E. I. Zamulaeva, E. A. Levashov, A. E. Kudryashov, P. V. Vakaev, and M. I. Petrzhik, Electrospark coatings deposited onto an Armco iron substrate with nano-and microstructured WC-Co electrodes: Deposition process, structure, and properties, Surf. Coat. Tech. 202, 3715-3722 (2008).

6. E. Rimini, R. D. Bastiani, E. Carria, M. G. Grimaldi, G. Nicotra, C. Bongiorno, and C. Spinella, Crystallization of sputtered-deposited and ion implanted amorphous $\mathrm{Ge}_{2} \mathrm{Sb}_{2} \mathrm{Te}_{5}$ thin films, J. Appl. Phys. 105, 123502 (2009).

7. H. Dong, and T. Bell, State-of-the-art overview: ion beam surface modification of polymers towards improving tribological properties, Surf. Coat. Tech.111, 29-40 (1999).

8. D. D. Dibtonto, P. T. Eubank, M. R. Patel, and M. A. Barrufet, Theoretical models of the electrical discharge machining process. I. A simple cathode erosion model, J. Appl. Phys. 66, 4095-4103 (1989).

9. T. H. Maiman, Optical and microwave-optical experiments in Ruby, Phys. Rev. Lett. 4, 564-566 (1960). 
10. T. H. Maiman, Stimulated optical radiation in Ruby, Nature 187, 493-494 (1960).

11. T. H. Maiman, Stimulated optical emission in fluorescent solids. I. theoretical considerations, Phys. Rev. 123, 1145-1150 (1961).

12. W. T. Walter, N. Solimene, M. Piltch, and G. Gould, 6C3-efficient pulsed gas discharge lasers, IEEE J. Quantum Elect. QE-2, 474-479 (1966).

13. D. Bäuerle, "laser Processing and Chemistry", Springer-Verlag: Berlin, Heidelberg (2000).

14. R. Menzel, "Photonics Linear and Nonlinear Interactions of Laser Light and Matter", Springer-Verlag Berlin, Heidelberg (2001).

15. H. Helvajian, Process control in laser material processing for the micro and nanometer scale domains, in Laser Precision Microfabrication, Springer Series in Materials Science, Vol. 135, Edited by K. Sugioka, M. Meunier, and A. Piquè, (Springer-Verlag Berlin Heidelberg, 2010), pp 1-30.

16. M. D. Shirk, and P. A. Molian, A review of ultrashort pulsed laser ablation of materials, $J$. Laser Appl. 10, 18-28 (1998).

17. M. S. Brown, and C. B. Arnold, Fundamentals of laser-material interaction and application to multiscale surface modification, in Laser Precision Microfabrication, Springer Series in Materials Science, Vol. 135, Edited by K. Sugioka, M. Meunier, and A. Piquè, (Springer Verlag Berlin Heidelberg, 2010), pp 91-120.

18. A. Y. Vorobyev, and C. Guo, Direct femtosecond laser surface nano/microstructuring and its applications, Laser Photonics Rev. 7, 385-407 (2013).

19. J. Bèkèsi, J.-H. Klein-Wiele, and P. Simon, Efficient submicron processing of metals with femtosecond UV pulses, Appl. Phys. A 76, 355-357(2003).

20. F. Korte, J. Serbin, J. Koch, A. Egbert, C. Fallnich, A. Ostendorf, and B. N. Chichkov, Towards nanostructuring with femtosecond laser pulses, Appl. Phys. A 77, 229-235 (2003). 
21. J. R. Vàzquez de Aldana, P. Moreno, and L. Roso, Ultrafast lasers: A new frontier for optical materials processing, Opt. Mater. 34, 572-578 (2012).

22. R. Kodama, P. A. Norreys, K. Mima, A. E. Dangor, R. G. Evans, H. Fujita, Y. Kitagawa, K. Krushelnick, T. Miyakoshi, N. Miyanaga, T. Norimatsu, S. J. Rose, T. Shozaki, K. shigemori, A. Sunahara, M. Tampo, K. A. Tanaka, Y. Toyama, T. Yamanaka, and M. Zepf, Fast heating of ultrahigh-density plasma as a step towards laser fusion ignition, Nature $\mathbf{4 1 2}$, 798-802 (2001).

23. N. M. Bulgakova, and A. V. Bulgakov, Pulsed laser ablation of solids: transition from normal vaporization to phase explosion, Appl. Phys. A 73, 199-208 (2001).

24. C. $\mathrm{Wu}$, and L. V. Zhigilei, Microscopic mechanisms of laser spallation and ablation of metal targets from large-scale molecular dynamics simulations, Appl. Phys. A 114, 11-32 (2014).

25. T. V. Kononenko, S. V. Garnov, S. M. Klimentov, V. I. Konov, E. N. Loubnin, F. Dausinger, A. Raiber, and C. Taut, Laser ablation of metals and ceramics in picosecondnanosecond pulsewidth in the presence of different ambient atmospheres, Appl. Surf. Sci. 109/110, 48-51 (1997).

26. B. Sallè, C. Chalèard, V. Detalle, J. L. Lacour, P. Mauchien, C. Nouvellon, and A. Semerok, Laser ablation efficiency of metal samples with UV laser nanosecond pulses, Appl. Surf. Sci.138-139, 302-305 (1999).

27. B. J. Garrison, and R. Srinivasan, Laser ablation of organic polymers: Microscopic models for photochemical and thermal processes, J. Appl. Phys. 57, 2909-2914 (1985).

28. B. N. Chichkov, C. Momma, S. Nolte, F. Von Alvensleben, and A. Tünnermann, Femtosecond, picosecond and nanosecond laser ablation of solids, Appl. Phys. A 63, 109115 (1996).

29. P. Simon, and J. Ihlemann, Ablation of submicron structures on metals and semiconductors by femtosecond UV-laser pulses, Appl. Surf. Sci. 109/110, 25-29 (1997). 
30. X. Zhu, A. Yu. Naumov, D. M. Villeneuve, and P. B. Corkum, Influence of laser parameters and material properties on micro drilling with femtosecond laser pulses, Appl. Phys. A 69, S367-S371 (1999).

31. J. H. Yoo, S. H. Jeong, R. Greif, and R. E. Russo, Explosive change in crater properties during high power nanosecond laser ablation of silicon, J. Appl. Phys. 88, 1638-1649 (2000).

32. R. L. Harzic, D. Breitling, M. Weikert, S. Sommer, C. Föhl, S. Valette, C. Donnet, E. Audouard, and F. Dausinger, Pulse width and energy influence on laser micromachining of metals in a range of 100 fs to 5 ps, Appl. Surf. Sci. 249, 322-331 (2005).

33. A. Y. Vorobyev, and C. Guo, Enhanced absorptance of gold following multipulse femtosecond laser ablation, Phys. Rev. B 72, 195422 (2005).

34. S. Nolte, B. N. Chichkov, H. Welling, Y. Shani, K. Lieberman, and H. Terkel, Nanostructuring with spatially localized femtosecond laser pulses, Opt. Lett. 24, 914-916 (1999).

35. A. Chimmalgi, T. Y. Choi, C. P. Grigoropoulos, and K. Komvopoulos, Femtosecond laser aperturless near-field nanomachining of metals assisted by scanning probe microscopy, Appl. Phys. Lett. 82, 1146-1148 (2003).

36. A. Plech, P. leiderer, and J. Boneberg, Femtosecond laser near field ablation, Laser \&Photon. Rev. 3, 435-451 (2009).

37. V. Zorba, X. Mao, and R. E. Russo, Laser wavelength effects in ultrafast near-field laser nanostructuring of Si, Appl. Phys. Lett. 95, 041110 (2009).

38. C. Wu, C. H. Crouch, L. Zhao, J. E. Carey, R. Younkin, J. A. Levinson, E. Mazur, R. M. Farrell, P. Grthoskar, and A. Karger, Near-unity below-band-gap absorption by microstructured silicon, Appl. Phys. Lett. 78, 1850-1852 (2001). 
39. A. Pereira, A. Cros, P. Delaporte, S. Georgiou, A. Manousaki, W. Marine, and M. Sentis, Surface nanostructuring of metals by laser irradiation: effects of pulse duration, wavelength and gas atmosphere, Appl. Phys. A 79, 1433-1437 (2004).

40. S. Amoruso, G. Ausanio, R. Bruzzese, M. Vitiello, and X. Wang, Femtosecond laser pulse irradiation of solid targets as a general route to nanoparticle formation in a vacuum, Phys. Rev. B 71, 033406 (2005).

41. A. V. Kabashin, M. Charbonneau-Lefort, M. Meunier, and R. Leonelli, Effects of deposition and post-fabrication conditions on photoluminescent properties of nanostructured $\mathrm{Si} / \mathrm{SiOx}$ films prepared by laser ablation, Appl. Surf. Sci. 168, 328-331 (2000).

42. S. Eliezer, N. Eliaz, E. Grossman, D. Fisher, I. Gouzman, Z. Henis, S. Pecker, Y. Horovitz, M. Fraenkel, S. Maman, and Y. Lereah, Synthesis of nanoparticles with femtosecond laser pulses, Phys. Rev. B 69, 144119 (2004).

43. A. Y. Vorobyev, and C. Guo, Femtosecond laser nanostructuring of metals, Opt. Express 14, 2164-2169 (2006).

44. A. Y. Vorobyev, and C. Guo, Femtosecond laser structuring of titanium implants, Appl. Surf. Sci. 253, 7272-7280 (2007).

45. Y. P. Meshcheryakov, and N. M. Bulgakova, Thermoelastic modeling of microbump and nanojet formation on nanosize gold films under femtosecond laser irradiation, Appl. Phys. A 82, 363-368 (2006).

46. D. S. Ivanov, B. Rethfeld, G. M. O’Connor, T. J. Glynn, A. N. Volkov, and L. V. Zhigilei, The mechanism of nanobump formation in femtosecond pulse laser nanostructuring of thin metal films, Appl. Phys. A 92, 791-796 (2008).

47. D. Eversole, B. Luk'yanchuk, and A. Ben-Yakar, Plasmonic laser nanoablation of silicon by the scattering of femtosecond pulses near gold nanospheres, Appl. Phys. A 89, 283291(2007). 
48. Y. Nakata, T. Okada, and M. Maeda, Lithographical laser ablation using femtosecond laser, Appl. Phys. A 79, 1481-1483 (2004).

49. K. Paivasaari, J. J. J. Kaakkunen, M. Kuittinen, and T. Jaaskelainen, Enhanced optical absorptance of metals using interferometric femtosecond ablation, Opt. Express 15, 1383813843 (2007).

50. C. Wang, Y.-C. Chang, J. Yao, C. Luo, S. Yin, P. Ruffin, C. Brantley, and E. Edwards, Surface enhanced Raman spectroscopy by interfered femtosecond laser created nanostructures, J. Appl. Lett. 100, 023107 (2012).

51. X. Li, D. Feng, T. Jia, Z. Sun, and Z. Xu, Fabrication of complex periodic micro/nanopatterns by multiple interfered femtosecond laser pulses on thin solid films, Micro \& Nano Letters 6, 177-180 (2011).

52. M. Birnbaum, Semiconductor Surface Damage Produced by Ruby Lasers, J. Appl. Phys. 36, 3688-3689 (1965).

53. D. C. Emmony, R. P. Howson, and L. J. Willis, Laser mirror damage in germanium at 10.6 m, Appl. Phys. Lett. 23, 598-600 (1973).

54. J. Perrière, C. Boulmer-Leborgne, R. Benzerga, and S. Tricot, Nanoparticle formation by femtosecond laser ablation, J. Phys. D: Appl. Phys.40, 7069-7076 (2007).

55. E. Stratakis, V. Zorba, M. Barberoglou, C. Fotakis, and G. A. Shafeev, Laser writing of nanostructures on bulk Al via its ablation in liquids, Nanotechnology 20, 105303 (2009).

56. E. T. Karim, M. Shugaev, C. Wu, Z. Lin, R. F. Hainsey, and L. V. Zhigilei, Atomistic simulation study of short pulse laser interactions with a metal target under conditions of spatial confinement by a transparent overlayer, J. Appl. Phys. 115, 183501 (2014).

57. J.-H. Klein-Wiele, and P. Simon, Sub-100nm pattern generation by laser direct writing using a confinement layer, Opt. Express 21, 9017-9023 (2013). 
58. Y. L. Page, and Paul Saxe, Symmetry-general least-squares extraction of elastic data for strained materials from ab initio calculations of stress, Phys. Rev. B 65, 104104 (2002).

59. C. Wu, E. T. Karim, A. N. Volkov, and L. V. Zhigilei, Atomic movies of laser-induced structural and phase transformations from molecular dynamics simulations, in Lasers in Materials Science, Springer Series in Materials Science, Vol. 191, Edited by M. Castillejo, P. M. Ossi, and L. V. Zhigilei (Springer International Publishing Switzerland, 2014), pp. 67-100.

60. E. T. Karim, C. Wu, and L. V. Zhigilei, Molecular dynamics simulations of laser-materials interactions: General and material-specific mechanisms of material removal and generation of crystal defects, in Fundamentals of laser-Assisted Micro-and Nanotechnologies Springer Series in Materials Science, Vol. 195, Edited by V. P. Veiko, and V. I. Konov (Springer International Publishing Switzerland, 2014), pp. 27-49.

61. F. Bottin, and G. Zèrah, Formation enthalpies of monovacancies in aluminum and gold under the condition of intense laser irradiation, Phys. Rev. B 75, 174114 (2007).

62. P.B. Hillyard, D.A. Reis, and K.J. Gaffney, Carrier-induced disordering dynamics in InSb studied with density functional perturbation theory, Phys. Rev. B 77, 195213 (2008).

63. P.L. Silvestrelli, A. Alavi, M. Parrinello, and D. Frenkel, Ab initio Molecular Dynamics Simulation of Laser Melting of Silicon, Phys. Rev. Lett. 77, 3149-3152 (1996).

64. J.S. Graves, and R.E. Allen, Response of GaAs to fast intense laser pulses, Phys. Rev. B 58, 13627 (1998).

65. T. Dumitrica, A. Burzo, Y. Dou, and R.E. Allen, Response of Si and InSb to ultrafast laser pulses, Phys. Status Solidi B 241, 2331-2342 (2004).

66. B.R. Torralva, and R.E. Allen, Mechanisms for laser control of chemical reactions, J. Mod. Opt. 49, 593-625 (2002). 
67. Y. Dou, B.R. Torralva, and R.E. Allen, Interplay of electronic and nuclear degrees of freedom in a femtosecond-scale photochemical reaction, Chem. Phys. Lett. 392, 352-357 (2004).

68. H.O. Jeschke, M.E. Garcia, M. Lenzner, J. Bonse, J. Krüger, and W. Kautek, Laser ablation thresholds of silicon for different pulse durations: theory and experiment, Appl. Surf. Sci. 197-198, 839-844 (2002).

69. Z. Lin, and R.E. Allen, Ultrafast equilibration of excited electrons in dynamical simulations, J. Phys.: Condens. Matter 21, 485503 (2009).

70. A.J. Neukirch, Z.Y. Guo, and O.V. Prezhdo, Time-Domain Ab Initio Study of PhononInduced Relaxation of Plasmon Excitations in a Silver Quantum Dot, J. Phys. Chem. C 116, 15034-15040 (2012).

71. R. M. More, K. H. Warren, D. A. Young, and G. B. Zimmerman, A new quotidian equation of state (QEOS) for hot dense matter, Phys. Fluids 31, 3059-3078 (1988).

72. K. Eidmann, J. Meyer-ter-Vehn, T. Schlegel, and S. Hüller, Hydrodynamic simulation of subpicosecond laser interaction with solid-density matter, Phys. Rev. E 62, 1202-1214 (2000).

73. T. E. Glover, Hydrodynamics of particle formation following femtosecond laser ablation, $J$. Opt. Soc. Am. B 20, 125-131 (2003).

74. J. P. Colombier, P. Combis, F. Bonneau, R. Le Harzic, and E. Audouard, Hydrodynamic simulations of metal ablation by femtosecond laser irradiation, Phys. Rev. B 71, 165406 (2005).

75. M. E. Povarnitsyn, K. V. Khishchenko, and P. R. Levashov, Phase transitions in femtosecond laser ablation, Appl. Surf. Sci. 225, 5120-5124 (2009).

76. M. E. Povarnitsyn, T. E. Itina, P. R. Levashov, and K. V. Khishchenko, Mechanisms of nanoparticle formation by ultra-short laser ablation of metals in liquid environment, Phys. Chem. Chem. Phys. 15, 3108-3114 (2013). 
77. A. N. Volkov, C. Sevilla, and L. V. Zhigilei, Numerical modeling of short pulse laser interaction with Au nanoparticle surrounded by water, Appl. Surf. Sci. 253, 6394-6399 (2007).

78. J. M. Sun, B. S. Gerstman, and B. Li, Bubble dynamics and shock waves generated by laser absorption of a photoacoustic sphere, J. Appl. Phys. 88, 2352-2362 (2000).

79. A. N. Volkov, and L. V. Zhigilei, Hydrodynamic multi-phase model for simulation of laser-induced non-equilibrium phase transformations, J. Phys.: Conference Series 59, 640645 (2007).

80. C. L. Liu, J. N. Leboeuf, R. F. Wood, D. B. Geobegan, J. M. Donato, K. R. Chen, and A. A. Puretzky, Computational modeling of physical processes during laser ablation, Mat. Sci. Eng. B 47, 70-77 (1997).

81. Z. Lin, and L. V. Zhigilei, Thermal excitation of d band electrons in Au: implications for laser-induced phase transformations, SPIE 6261, 62610U (2006).

82. J. H. Bechtel, Heating of solid targets with laser pulses, J. Appl. Phys. 46, 1585-1593 (1975).

83. M. Sparks, Theory of laser heating of solids: Metals, J. Appl. Phys. 47,837-849 (1976).

84. G. L. Eesley, Generation of nonequilibrium electron and lattice temperatures in copper by picosecond laser pulses, Phys. Rev. B 33, 2144-2151 (1986).

85. P. B. Corkum, F. Brunel, N. K. Sherman, and T. Srinivasan-Rao, Thermal Response of Metals to Ultrashort-Pulse Laser Excitation, Phys. Rev. Lett. 61, 2886-2889 (1988).

86. Z. Lin, L. V. Zhigilei, and V. Celli, Electron-phonon coupling and electron heat capacity of metals under conditions of strong electron-phonon nonequilibrium, Phys. Rev. B 77, 075133 (2008). 
87. J. Hohlfeld, S.-S. Wellershoff, J. Güdde, U. Conrad, V. Jähnke, and E. Mattias, Electron and lattice dynamics following optical excitation of metals, Chem. Phys. 251, 237-258 (2000).

88. Z. Lin, and L. V. Zhigilei, Temperature dependences of the electron-phonon coupling, electron heat capacity and thermal conductivity in Ni under femtosecond laser irradiation, Appl. Surf. Sci.253, 6295-6300 (2007).

89. W. Kautek, and O. Armbruster, Non-Thermal Material Response to Laser Energy Deposition., in Lasers in Materials Science, Springer Series in Materials Science, Vol. 191, Edited by M. Castillejo, P. M. Ossi, and L. V. Zhigilei (Springer International Publishing Switzerland, 2014), pp. 43-66.

90. S. I. Anisimov, B. L. Kapeliovich, and T. L. perel'man, Electron emission from metal surfaces exposed to ultrashort laser pulses, Sov. Phys. JETP 39, 375-377 (1974).

91. S.-S. Wellershoff, J. Hohlfeld, J. Güdde, and E. Matthias, The role of electron-phonon coupling in femtosecond laser damage of metals, Appl. Phys. A: Mater Sci. Process. 69, S99-S107 (1999).

92. A. N. Smith and P. M. Norris, Influence of intraband transitions on the electron thermoreflectance response of metals, Appl. Phys. Lett. 78, 1240-1242 (2001).

93. J. L. Hostetler, A. N. Smith, and P. M. Norris, Simultaneous Measurement of Thermophysical and Mechanical Properties of Thin Films, Int. J. Thermophys. 19, 569-577 (1998).

94. P. M. Norris, A. P. Caffrey, R. J. Stevens, J. M. Klopf, J. T. McLeskey, Jr., and A. N. Smith, Femtosecond pump-probe nondestructive examination of materials (invited), Rev. Sci. Instrum. 74, 400-406 (2003).

95. H. E. Elsayed-Ali, T. Juhasz, G. O. Smith, and W. E. Bron, Femtosecond thermoreflectivity and thermotransmissivity of polycrystalline and single-crystalline gold films, Phys. Rev. B 43, 4488-4491 (1991). 
96. J. L. Hostetler, A. N. Smith, D. M. Czajkowsky, and P. M. Norris, Measurement of the electron-phonon coupling factor dependence on film thickness and grain size in $\mathrm{Au}, \mathrm{Cr}$, and Al, Appl. Opt. 38, 3614-3620 (1999).

97. J. H. Hodak, A. Henglein, and G. V. Hartland, Electron-phonon coupling dynamics in very small (between 2 and $8 \mathrm{~nm}$ diameter) Au nanoparticles, J. Chem. Phys. 112, 5942-5947 (2000).

98. N. D. Fatti, and F. Vallèe, Ultrafast electron interactions in metal clusters, C. R. Phys. 3, 365-380 (2002).

99. P. J. Antaki, Technical Note Importance of nonequilibrium thermal conductivity during short-pulse laser-induced desorption from metals, Int. J. Heat Mass Transfer 45, 4063-4067 (2002).

100. V. Schmidt, W. Husinsky, and G. Betz, Ultrashort laser ablation of metals: pump-probe experiments, the role of ballistic electrons and the two-temperature model, Appl. Surf. Sci. 197, 145-155 (2002).

101. T. Q. Qiu, and C. L. Tien, Size Effects on nonequilibrium laser heating of metal films, J. Heat Transfer. 115, 842-847 (1993).

102. L. A. Falkovsky, and E. G. Mishchenko, Electron-lattice kinetics of metals heated by ultrashort laser pulses, JETP 115, 149-157 (1999).

103. J. K. Chen, J. E. Beraun, L. E. Grimes, and D. Y. Tzou, Modeling of femtosecond laserinduced non-equilibrium deformation in metal films, Int. J. Solids Struct. 39, 3199-3216 (2002).

104. B. J. Alder, and T. E. Wainwright, Phase transition for a hard sphere system, J. Chem. Phys. 27, 1208-1209 (1957).

105. J. C. Butcher, Numerical methods for ordinary differential equations in the 20th century, $J$. Comp. Appl. Math. 125 1-29 (2000). 
106. L. Verlet, Computer "Experiments" on classical fluids. I. Thermodynamical properties of Lennard-Jones molecules, Phys. Rev. 159, 98-103 (1967).

107. M. P. Allen, and D. J. Tildesley "Computer Simulation of Liquids", Clarendon Press: Oxford (1987).

108. J. F. Lutsko, D. Wolf, S. R. Phillpot, and S. Yip, Molecular-dynamics study of latticedefect-nucleated melting in metals using an embedded-atom-method potential, Phys. Rev. B 40, 2841-2855 (1989).

109. X. Wang, and X. Xu, Molecular dynamics simulation of thermal and thermosmechanical phenomena in picosecond laser material interaction, Int. J. Heat Mass Transfer, 46, 45-53 (2003).

110. L. V. Zhigilei, and B. J. Garrison, Pressure waves in microscopic simulations of laser ablation, in: Multiscale Modelling of Materials, edited by T. Diaz de la Rubia, T. Kaxiras, V. Bulatov, N.M.Ghoniem, and R. Phillips, (Mater. Res. Soc. Symp. Proc. 538), 491-496 1999

111. J. I. Etcheverry, and M. Mesaros, Molecular dynamics simulation of the production of acoustic waves by pulsed laser irradiation, Phys. Rev. B 60, 9430-9434 (1999).

112. R. F. W. Herrmann, J. Gerlach, and E. E. B. Campbell, Ultrashort pulse laser ablation of silicon: an MD simulation study, Appl. phys. A: Mater. Sci. Process. 66, 35-42 (1998).

113. L. V. Zhigilei, and B. J. Garrison, Microscopic mechanisms of laser ablation of organic solids in the thermal and stress confinement irradiation regimes, J. Appl. Phys. 88, 12811298 (2000).

114. L. V. Zhigilei, Dynamics of the plume formation and parameters of the ejected clusters in short-pulse laser ablation, Appl. Phys. A: Mater. Sci. Process. 76, 339-350 (2003).

115. M. W. Finnis, P. Agnew, and A. J. E. Foreman, Thermal excitation of electrons in energetic displacement cascades, Phys. Rev. B 44, 567-574 (1991). 
116. H. Häkkinen, and U. Landman, Superheating melting and annealing of Copper surface, Phys. Rev. Lett. 71, 1023-1026 (1993).

117. C. Schäfer, H. M. Urbassek, and L.V. Zhigilei, Metal ablation by picosecond laser pulse: A hybrid simulation, Phys. Rev. B 66, 115404 (2002).

118. D. S. Ivanov, and L. V. Zhigilei, Combined atomistic-continuum modeling of short-pulse laser melting and disintegration of metal films, Phys. Rev. B 68, 064114 (2003).

119. L. V. Zhigilei, Z. Lin, and D. S. Ivanov, Molecular dynamics study of short pulse laser melting, recrystallization, spallation, and ablation of metal targets, ASME paper IMECE2006-16305, (2006).

120. L. V. Zhigilei, Z. Lin, and D. S. Ivanov, Atomistic Modeling of Short Pulse Laser Ablation of Metals: Connections between Melting, Spallation, and Phase Explosion, J. Phys. Chem. C 113, 11892-11906 (2009).

121. L. V. Zhigilei, Z. Lin, D. S. Ivanov, E. Leveugle, W. H. Duff, D. Thomas, C. Sevilla, and S. J. Guy, Atomic/molecular-level simulations of laser-materials interactions, in LaserSurface Interactions for New Materials Production: Tailoring Structure and Properties, Springer Series in Materials Science, Vol. 130, Edited by A. Miotello and P. M. Ossi (Springer Verlag: New York, 2010), pp. 43-79.

122. N. W. Ashcroft, and N. D. Mermin, "Solid State Physics”, Holt, Rinehart and Winston, New York (1976).

123. http://www.faculty.virginia.edu/CompMat/electron-phonon-coupling.

124. J. K. Chen, and J. E. Beraun, Numerical study of ultrashort laser pulse interactions with metal films, Num. Heat Transfer, Part A 40, 1-20 (2001).

125. R. H. M. Groeneveld, R. Sprik, and A. Lagendijk, Femtosecond spectroscopy of electronelectron and electron-phonon energy relaxation in Ag and $\mathrm{Au}$, Phys. Rev. B 51, 1143311445 (1995). 
126. K. C. Mills, B. J. Monaghan, and B. J. Keene, Thermal conductivities of molten metals: Part 1 pure metals, Int. Mater. Rev. 41, 209-242 (1996).

127. S. I. Anisimov, and B. Rethfeld, Theory of ultrashort laser pulse interaction with a metal, Proc. SPIE 3039, 192 (1997).

128. S. M. Foiles, M. I. Baskes, and M. S. Daw, Embedded-atom-method functions for the fcc metals Cu, Ag, Au, Ni, Pd, Pt, and their alloys, Phys. Rev. B 33, 7983-7991 (1986).

129. Z. Lin, R. A. Johnson, and L. V. Zhigilei, Computational study of the generation of crystal defects in a bcc metal target irradiated by short laser pulses, Phys. Rev. B 77, 214108 (2008).

130. C. Schäfer, H. M. Urbassek, L. V. Zhigilei, and B. J. Garrison, Pressure-transmitting boundary conditions for molecular-dynamics simulations, Comp. Mater. Sci. 24, 421-429 (2002).

131. J. Limpert, O. Schmidt, J. Rothhardt, F. Röser, T. Schreiber, A. Tünnermann, S. Ermeneux, P. Yvernault, and F. Salin, Extended single-mode photonic crystal fiber lasers, Opt. Express 14, 2715-2720 (2006).

132. R. J. Levis, G. M. Menkir, and H. Rabitz, Selective bond dissociation and rearrangement with optimally tailored, strong-field laser pulses, Science 292, 709-713 (2001).

133. F. Korte, S. Adams, A. Egbert, C. Fallnich, A. Ostendorf, S. Nolte, M. Will, J.-P. Ruske, B. N. Chichov, and A. Tünnermann, Sub-diffraction limited structuring of solid targets with femtosecond laser pulses, Opt. Express 7, $41-49$ (2000).

134. E. T. Karim, Z. Lin, and L. V. Zhigilei, Molecular dynamics study of femtosecond laser interactions with Cr targets, AIP Conf. Proc. 1464, 280-293 (2012).

135. R. L. Harzic, N. Huot, E. Audouard, C. Jonin, P. Laporte, S. Valette, A. Fraczkiewicz, and R. Fortunier, Comparison of heat-affected zones due to nanosecond and femtosecond laser pulses using transmission electronic microscopy, Appl. Phys. Lett. 80, 3886-3888, (2002). 
136. V. Margetic, K. Niemax, and R. Hergenröder, Application of femtosecond laser ablation time-of-flight mass spectrometry to in-depth multilayer analysis, Anal. Chem. 75, 34353439 (2003).

137. A. I. Kuznetsov, J. Koch, and B. N. Chichkov, Nanostructuring of thin gold films by femtosecond lasers, Appl. Phys. A: Mater. Sci. Process. 94, 221-230 (2009).

138. D. Hwang, S.-G. Ryu, N. Misra, H. Jeon, and C. P. Grigoropoulos, Nanoscale laser processing and diagnostics, Appl. Phys. A: Mater. Sci. Process. 96, 289-306 (2009).

139. R. R. Gattass, and E. Mazur, Femtosecond laser micromachining in transparent materials, Nature Photonics 2, 219-225 (2008).

140. R. Kelly, and A. Miotello, Comments on explosive mechanisms of laser sputtering, Appl. Surf. Sci. 96-98, 205-215 (1996).

141. C. Wu, D. A. Thomas, Z. Lin, and L. V. Zhigilei, Runaway lattice-mismatched interface in an atomistic simulation of femtosecond laser irradiation of $\mathrm{Ag}$ film- $\mathrm{Cu}$ substrate system, Appl. Phys. A: Mater. Sci. Process. 104, 781-792 (2011).

142. W. L. Chan, R. S. Averback, D. G. Cahill, and Y. Ashkenazy, solidification Velocities in Deeply Undercooled Silver, Phys. Rev. Lett. 102, 095701 (2009).

143. D. S. Ivanov, and L. V. Zhigilei, Kinetic Limit of Heterogeneous Melting in Metals, Phys. Rev. Lett. 98, 195701 (2007).

144. M. M. Martynyuk, Mechanism for metal damage by intense electromagnetic radiation, Sov. Phys. Tech. Phys. 21, 430-433 (1976).

145. A. Miotello, and R. Kelly, Laser-induced phase explosion: new physical problems when a condensed phase approaches the thermodynamic critical temperature, Appl. Phys. A: Mater. Sci. Process. 69, S67-S73 (1999).

146. C. Cheng, and X. Xu, Mechanisms of decomposition of metal during femtosecond laser ablation, Phys. Rev. B 72, 165415 (2005). 
147. M. E. Povarnitsyn, T. E. Itina, M. Sentis, K. V. Khishchenko, and P. R. Levashov, Material decomposition mechanisms in femtosecond laser interactions with metals, Phys. Rev. B 75, 235414 (2007).

148. V. V. Semak, J. G. Thomas, and B. R. Campbell, Drilling of steel and HgCdTe with the femtosecond pulses produced by a commercial laser system, J. Phys. D: Appl. Phys. 37, 2925-2931 (2004).

149. G. Paltauf, and P. E. Dyer, Photomechanical Processes and Effects in Ablation, Chem. Rev. 103, 487-518 (2003).

150. J. Yang, Y. Zhao, N. Zhang, Y. Liang, and M. Wang, Ablation of metallic targets by highintensity ultrashort laser pulses, Phys. Rev. B 76, 165430 (2007).

151. M. B. Agranat, S. I. Anisimov, S. I. Ashitkov, V. V. Zhakhovskii, N. A. Inogamov, K. Nishihara, Yu. V. Petrov, V. E. Fortov, and V. A. Khokhlov, Dynamics of plume and crater formation after action of femtosecond laser pulse, Appl. Surf. Sci. 253, 6276-6282 (2007).

152. E. Leveugle, D. S. Ivanov, and L. V. Zhigilei, Photomechanical spallation of molecular and metal targets: molecular dynamics study, Appl. Phys. A 79, 1643-1655 (2004).

153. B. J. Demaske, V. V. Zhakhovsky, N. A. Inogamov, and I. I. Oleynik, Ablation and spallation of gold films irradiated by ultrashort laser pulses, Phys. Rev. B 82, 064113 (2010).

154. K. Sokolowski-Tinten, J. Bialkowski, M. Boing, A. Cavalleri, and D. von der Linde, Thermal and nonthermal melting of gallium arsenide after femtosecond laser excitation, Phys. Rev. B 58, R11805-R11808 (1998).

155. A. M. Lindenberg, S. Engemann, K. J. Gaffney, K. Sokolowski-Tinten, J. Larsson, P. B. Hillyard, D. A. Reis, D. M. Fritz, J. Arthur, R. A. Akre, M. J. George, A. Deb, P. H. Bucksbaum, J. Hajdu, D. A. Meyer, M. Nicoul, C. Blome, Th. Tschentscher, A. L. Cavalieri, R.W. Falcone, S. H. Lee, R. Pahl, J. Rudati, P. H. Fuoss, A. J. Nelson, P. Krejcik, D. P. Siddons, P. Lorazo, and J. B. Hastings, X-Ray Diffuse Scattering 
Measurements of Nucleation Dynamics at Femtosecond Resolution, Phys. Rev. Lett. 100, 135502 (2008).

156. V. Recoules, J. Clérouin, G. Zérah, P. M. Anglade, and S. Mazevet, Effect of Intense Laser Irradiation on the Lattice Stability of Semiconductors and Metals, Phys. Rev. Lett. 96, 055503 (2006).

157. H. O. Jeschke and M. E. Garcia, Ultrafast structural changes induced by femtosecond laser pulses, in Nonlinear Optics, Quantum Optics, and Ultrafast Phenomena with X-Rays, ed. B. W. Adams (Springer-Verlag, New York, 2003), pp. 175-214.

158. H. Masuhara, T. Asahi, and Y. Hosokawa, Laser nanochemistry, Pure Appl. Chem. 78, 2205-2226 (2006).

159. N. M. Bulgakova, R. Stoian, A. Rosenfeld, I. V. Hertel, W. Marine, and E. E. B. Campbell, A general continuum approach to describe fast electronic transport in pulsed laser irradiated materials: The problem of Coulomb explosion, Appl. Phys. A: Mater. Sci. Process. 81, 345-356 (2005).

160. L. V. Zhigilei, E. Leveugle, D. S. Ivanov, Z. Lin, and A. N. Volkov, Molecular dynamics simulations of short pulse laser ablation: Mechanisms of material ejection and particle generation, in Nanosized Material Synthesis by Action of High-Power Energy Fluxes on Matter (Siberian Branch of the Russian Academy of Sciences: Novosibirsk, 2010), pp. 147220, in Russian

161. Z. Lin, and L. V. Zhigilei, Time-resolved diffraction profiles and atomic dynamics in shortpulse laser-induced structural transformations: Molecular dynamics study, Phys. Rev. B 73, 184113 (2006).

162. Z. Lin, E. Leveugle, E. M. Bringa, and L. V. Zhigilei, Molecular Dynamics Simulation of Laser Melting of Nanocrystalline Au, J. Phys. Chem. C 114, 5686-5699 (2010).

163. Handbook of Optical Constants of Solids I/II, edited by D. Palik, Academic Press, London, (1985/1991). 
164. V. E. Fortov, V. V. Kostin, and S. Eliezer, Spallation of metals under laser irradiation, $J$. Appl. Phys. 70, 4524-4531 (1991).

165. H. Tamura, T. Kohama, K. Kondo, and M. Yoshida, Femtosecond-laser-induced spallation in aluminum, J. Appl. Phys. 89, 3520-3522 (2001).

166. B. J. Garrison, T. E. Itina, and L. V. Zhigilei, Limit of overheating and the threshold behavior in laser ablation, Phys. Rev. E 68, 041501 (2003).

167. E. Leveugle and L. V. Zhigilei, Molecular dynamics simulation study of the ejection and transport of polymer molecules in matrix-assisted pulsed laser evaporation, J. Appl. Phys. 102, 074914 (2007).

168. S. Amoruso, R. Bruzzese, C. Pagano, and X. Wang, Features of plasma plume evolution and material removal efficiency during femtosecond laser ablation of nickel in high vacuum, Appl. Phys. A: Mater. Sci. Process. 89, 1017-1024 (2007).

169. K. Sokolowski-Tinten, J. Bialkowski, A. Cavalleri, D. von der Linde, A. Oparin, J. Meyerter-Vehn, and S. I. Anisimov, Transient States of Matter during Short Pulse Laser Ablation, Phys. Rev. Lett. 81, 224-227 (1998).

170. N. A. Inogamov, Y. V. Petrov, S. I. Anisimov, A. M. Oparin, N. V. Shaposhnikov, D. von der Linde, and J. Meyer-ter-Vehn, JETP Lett. 69, 310-316 (1999).

171. L.V. Zhigilei, E. Leveugle, B.J. Garrison, Y.G. Yingling, M.I. Zeifman, Computer simulations of laser ablation of molecular substrates, Chem. Rev. 103, 321-347 (2003).

172. A. G. Zhidkov, L. V. Zhigilei, A. Sasaki, and T. Tajima, Short-laser-pulse Driven Emission of Energetic Ions into a Solid Target from a Surface Layer Spalled by a Laser Prepulse, Appl. Phys. A: Mater. Sci. Process. 73,741-747 (2001).

173. L. V. Zhigilei, D. S. Ivanov, E. Leveugle, B. Sadigh, and E. M. Bringa, Computer modeling of laser melting and spallation of metal targets, High-Power Laser Ablation V, edited by C. R. Phipps, Proc. SPIE 5448, 505-519 (2004). 
174. L. Zhang and X. Wang, Hybrid atomistic-macroscale modeling of long-time phase change in nanosecond laser-material interaction, Appl. Surf. Sci. 255, 3097-3103 (2008).

175. R. Sangiorgi, M. L. Muolo, and A. Passerone, Surface tension and adsorption in liquid Silver-Oxygen alloys, Acta metall. 30, 1597-1604 (1982).

176. W. B. Chung, K. Nogi, W. A. Miller, and A. Mclean, Surface tension of liquid Cr-O system, 33, 753-757 (1992).

177. W.H. Duff, and L.V. Zhigilei, Computational study of cooling rates and recrystallization kinetics in short pulse laser quenching of metal targets, J. Phys.: Conference Series 59, 413-417 (2007).

178. S. I. Anisimov, V. V. Zhakhovskii, N. A. Inogamov, K. Nishihara, and Yu. V. Petrov, Simulation of the expansion of a crystal heated by an ultrashort laser pulse, Appl. Surf. Sci., 253, 6390-6393 (2007).

179. D. S. Ivanov, Z. Lin, B. Rethfeld, G. M. O’Connor, T. J. Glynn, and L. V. Zhigilei, Nanocrystalline structure of nanobump generated by localized photoexcitation of metal film , J. Appl. Phys. 107, 013519 (2010).

180. X. Wang, and Y. Lu, Solidification and epitaxial regrowth in surface nanostructuring with laser-assisted scanning tunneling microscope, J. Appl. Phys., 98, 114304 (2005).

181. E. Ohmura, I. Fukumoto, I. Miyamoto, Molecular dynamics simulation on laser ablation of metal and silicon, Int. J. Japan Soc. Prec. Eng. 32, 248-253 (1998).

182. X. W. Zhou, H. N. G. Wadley, R. A. Johnson, D. J. Larson, N. Tabat, A. Cerezo, A. K. Petford-Long, G. D. W. Smith, P. H. Clifton, R. L. Martens, and T. F. Kelly, Atomic scale structure of sputtered metal multilayers, Acta Mater. 49, 4005-4015 (2001).

183. R. A. Johnson, Interstitials and vacancies in $\alpha$ Iron, Phys. Rev. 134, A1329 (1964).

184. G. J. Ackland, and R. Thetford, An improved $N$-body semi-empirical model for bodycentred cubic transition metals, Philos. Mag. A 56, 15-30 (1987). 
185. M. Kiritani, Similarity and dierence between fcc, bcc, hcp metals from the view point of point defects cluster formation, J. Nucl. Mater. 276, 41-49 (2000).

186. H. Ohkubo, Z. Tang, Y. Nagai, M. Hasegawa, T. Tawara, and M. Kiritani, Positron annihilation study of vacancy-type defects in high-speed deformed $\mathrm{Ni}, \mathrm{Cu}$, and $\mathrm{Fe}, \mathrm{Mat}$. Sci. Eng. A 350, 95-101 (2003).

187. R. A. Johnson, Alloy models with the embedded-atom method, Phys. Rev. B 39, 12554 (1989)

188. C. Wu, M. S. Christensen, J.-M. Savolainen, P. Balling, and L. V. Zhigilei, Generation of sub-surface voids and a nanocrystalline surface layer in femtosecond laser irradiation of a single crystal Ag target, Phys. Rev. B 91, 035413 (2015).

189. E. T. Karim, M. S. Shugaev, C. Wu, Z. Lin, H. Matsumoto, M. Conneran, J. Kleinert, R. F. Hainsey, and L. V. Zhigilei, Experimental characterization and atomistic modeling of interfacial void formation and detachment in short pulse laser-processing of metal surfaces covered by transparent overlayers, Appl. Phys. A, submitted.

190. J. B. Cohen, R. Hinton, K. Lay, and S. Sass, Partial dislocations on the $\{110\}$ planes in the b.c.c. lattice, Acta Matall, 10, 894-895 (1962).

191. V. Vìtek, On the stability of stacking faults in B.C.C. crystals, Philos. Mag. 21, 1275-1278 (1970).

192. V. Vìtek, Intrinsic stacking faults in body-centered cubic crystals, Philos. Mag. 18, 773786 (1968).

193. R. P. Steijn, and R. M. Brick, Flow and Fracture of Single Crystals of High-Purity Ferrites, Trans. Amer. Soc. Met. 46, 1406 (1954).

194. Y. An, and H. Jiang, A finite element simulation on transient large deformation and mass diffusion in electrodes for lithium ion batteries, Modeling Simul. Mater. Sci. Eng., 21, 074007 (2013). 
195. D. Hull, and D. J. Bacon, “Introduction to Dislocation”, International Series on Materials Science and Technology, V 37, Elsevier Science Inc. (1984).

196. A. Stukowski, and K. Albe, Extracting dislocations and non-dislocation crystal defects from atomic simulation data, Modeling Simul. Mater. Sci. Eng. 18, 085001 (2010).

197. A. Stukowski, V. V. Bulatov, and A. Arsenlis, Automated identification and indexing of dislocations in crystal interfaces, Modeling Simul. Mater. Sci. Eng. 20, 085007 (20102).

198. A. Stukowski, Structure identification methods for atomistic simulations of crystalline materials, Modeling Simul. Mater. Sci. Eng. 20, 045021 (2012).

199. P. M. Derlet, D. Nguyen-Manh, and S. L. Dudarev, Multiscale modeling of crowdion and vacancy defects in body-centered-cubic transition metals, Phys. Rev. B 76, 054107 (2007).

200. D. Turnbull, Formation of crystal nuclei in Liquid Metals, J. Appl. Phys. 21, 1022-1028 (1950).

201. H. A. Wilson, On the velocity of solidification and viscosity of super-cooled liquids, Phil. Mag. 50, 238-250 (1900).

202. J. Frenkel, J. Phys. Z. Sow. 1, 498-500 (1932).

203. K. Nordlund, and R. S. Averback, Role of Self-Interstitial Atoms on the High Temperature Properties of Metals, Phys. Rev. Lett. 80, 4201-4204 (1998).

204. T. Egami, and D. Srolovitz, Local structure fluctuations in amorphous and liquid metals: a simple theory of the glass transition, J. Phys. F: Met. Phys. 12, 2141-2163 (1982).

205. K. A. Jackson, The interface kinetics of crystal growth process, Interface Sci. 10, 159-169 (2002).

206. Y. Ashkenazy, R. S. Averback, Kinetic stages in the crystallization of deeply undercooled body-centered-cubic and face-centered-cubic metals, Acta Mater 58, 524-530 (2010).

207. H. Schultz, Defect parameters of b.c.c, metals: group-specific trends, Materials Sci. Eng. A 141, 149-167 (1991). 
208. Y. Ashkenazy, and R. S. Averback, Atomic mechanisms controlling crystallization behavior in metals at deep undercooling, EPL, 79, 26005 (2007).

209. A. F. Voter, and S. P. Chen, Accurate interatomic potentials for Ni, Al, and Ni $3 \mathrm{Al}$, Mater. Res. Soc. Symp. Proc.82, 175-180 (1987).

210. D. A. Porter, K. E. Easterling, and M. Y. Sherif, "Phase Transformations in Metals and Alloys third edition”, CRC press Taylor and Francis group, Boca Raton (2009).

211. G. J. Ackland, and A. P. Jones, Applications of local crystal structure measures in experiment and simulation, Phys. Rev. B 73, 054104 (2006).

212. M. Volmer, and A. Weber, Nucleation of supersaturated structures, Z. phys. Chem. 119, 277-301 (1926).

213. D. Turnbull, and J. C. Fisher, Rate of Nucleation in Condensed Systems, J. Chem. Phys.17, 71-73 (1949).

214. D. Turnbull, and R. E. Cech, Microscopic Observation of the Solidification of Small Metal Droplets, J. Appl. Phys. 21, 804-810 (1950).

215. B. P. Fairand, B. A. Wilcox, W. J. Gallagher, and D. N. Williams, Laser shock-induced microstructural and mechanical property changes in 7075 aluminum, J. Appl. Phys. 43, 3893-3895 (1972).

216. R. Fabbro, J. Fournier, P. Ballard, D. Devaux, and J. Virmont, Physical study of laserproduced plasma in confined geometry, J. Appl. Phys. 68, 775-784 (1990).

217. P. Peyre and R. Fabbro, Laser shock processing: a review of the physics and applications, Opt. Quantum Electron. 27, 1213-1229 (1995).

218. X. Wu, Z. Duan, H. Song, Y. Wei, X. Wang, and C. Huang, Shock pressure induced by glass-confined laser shock peening: Experiments, modeling and simulation, J. Appl. Phys. 110, 053112 (2011). 
219. J. Bohandy, B. F. Kim, and F. J. Adrian, Metal deposition from a supported metal film using an excimer laser, J. Appl. Phys. 60, 1538-1539 (1986).

220. I. Zergioti, S. Mailis, N. A. Vainos, C. Fotakis, S. Chen, and C. P. Grigoropoulos, Microdeposition of metals by femtosecond excimer laser, Appl. Surf. Sci. 127-129, 601-605 (1998).

221. M. Domke, S. Rapp, M. Schmidt, and H. P. Huber, Ultra-fast movies of thin-film laser ablation, Appl. Phys. A 109, 409-420 (2012).

222. G. Heise, M. Englmaier, C. Hellwig, T. Kuznicki, S. Sarrach, and H. P. Huber, Laser ablation of thin molybdenum films on transparent substrates at low fluences, Appl. Phys. A 102, 173-178 (2011).

223. G. Heise, M. Domke, J. Konrad, S. Sarrach, J. Sotrop, and H. P. Huber, Laser lift-off initiated by direct induced ablation of different metal thin films with ultra-short laser pulses, J. Phys. D: Appl. Phys. 45, 315303 (2012).

224. N. C. Anderholm, laser-generated stress waves, Appl. Phys. Lett. 16, 113-115 (1970).

225. T. Yabe, C. Phipps, M. Yamaguchi, R. Nakagawa, K. Aoki, H. Mine, Y. Ogata, C. Baasandash, M. Nakagawa, E. Fujiwara, K. Yoshida, A. Nishiguchi, and I. Kajiwara, Microairplane propelled by laser driven exotic target, Appl. Phys. Lett. 80, 4318-4320 (2002).

226. J. Sotrop, A. Kersch, M. Domke, G. Heise, and H. P. Huber, Numerical simulation of ultrafast expansion as the driving mechanism for confined laser ablation with ultra-short laser pulses, Appl. Phys. A 113, 397-411 (2013).

227. M. V. Shugaev, and N. M. Bulgakova, Thermodynamic and stress analysis of laser induced forward transfer of metals, Appl. Phys. A 101, 103-109 (2010).

228. S. I. Anisimov, V. V. Zhakhovskii, N. A. Inogamov, K. Nishihara, A. M. Oparin, and Yu. V. Petrov, Destruction of a Solid Film under the Action of Ultrashort Laser Pulse, JETP Lett. 77, 606-610 (2003). 
229. A. K. Upadhyay and H. M. Urbassek, Melting and fragmentation of ultra-thin metal films due to ultrafast laser irradiation: a molecular-dynamics study, J. Phys. D: Appl. Phys. 38, 2933-2941 (2005).

230. B. J. Demaske, V. V. Zhakhovsky, N. A. Inogamov, and I. I. Oleynik, Ablation and spallation of gold films irradiated by ultrashort laser pulses, Phys. Rev. B 82, 064113 (2010).

231. D. S. Ivanov and L. V. Zhigilei, Effect of Pressure Relaxation on the Mechanisms of ShortPulse Laser Melting, Phys. Rev. Lett. 91, 105701 (2003).

232. C. F. Richardson and P. Clancy, Picosecond laser processing of Copper and Gold, Mol. Simul. 7, 335-355 (1991).

233. M. Gill-Comeau and L. J. Lewis, Ultrashort-pulse laser ablation of nanocrystalline aluminum, Phys. Rev. B 84, 224110 (2011).

234. C. Li, J. Zhang, and X. Wang, Phase change and stress wave in picosecond laser-material interaction with shock wave formation, Appl. Phys. A 112, 677-687 (2013).

235. "Handbook of Chemistry and Physics", edited by R. C. Weast, 64th ed. (CRC Press, Boca Raton, 1983.

236. R. W. Ohse, and H. von Tippelskirch, The critical constants of the elements and of some refractory materials with high critical temperatures (A review), High Temp.-High Press. 9, 367-385 (1977).

237. K. S. Gadre and T. L. Alford, Contact angle measurements for adhesion energy evaluation of silver and copper films on parylene-n and $\mathrm{SiO}_{2}$ substrates, J. Appl. Phys. 93, 919-923 (2003).

238. A. Vogel and V. Venugopalan, Mechanisms of pulsed laser ablation of biological tissues, Chem. Rev. 103, 487-518 (2003). 
239. J. Ihlemann, and R. Weichenhain-Schriever, Pulsed laser-induced formation of silica nanogrids, Nanoscale Res. Lett. 9, 102 (2014).

240. A. V. Smith, and B. T. Do, Bulk and surface laser damage of silica by picosecond and nanosecond pulses at $1064 \mathrm{~nm}$, Appl. Opt. 47, 4812-4832 (2008).

241. J. Cagnoux, and F. Longy, Spallation and shock-wave behavior of some ceramics, J. Phys. Colloq. 49 (C3), C3-3-C3-10 (1988).

242. J.-M. Savolainen, M. S. Christensen, P. Balling, Material swelling as the first step in the ablation of metals by ultrashort laser pulses, Phys. Rev. B 84, 193410 (2011).

243. J. V. Oboňa, V. Ocelík, J. C. Rao, J. Z. P. Skolski, G. R. B. E. Römer, A. J. Huis in 't Veld, J. Th. M. De Hosson, Modification of Cu surface with picosecond laser pulses, Appl. Surf. Sci. 303, 118-124 (2014).

244. S. Hermann, N.-P. Harder, R. Brendel, D. Herzog, and H. Haferkamp, Picosecond laser ablation of $\mathrm{SiO}_{2}$ layers on silicon substrates, Appl. Phys. A 99, 151-158 (2010).

245. C. Wu, M. S. Cheristensen, J-M. Savolainen, P. Balling, L. V. Zhigilei, Generation of subsurface voids and a nanocrystalline surface layer in femtosecond laser irradiation of a single crystal Ag target, Phys. Rev. B 91, 035413 (2015) 